\title{
Silver-Promoted $(4+1)$ Annulation of Isocyanoacetates with Alkylpyridinium Salts: \\ Divergent Regioselective Synthesis of 1,2-Disubstituted Indolizines
}

Yan Chen,$^{+}$Andrey Shatskiy, Jian-Quan Liu, ${ }^{+, \neq, *}$ Markus D. Kärkäs, ${ }^{\neq, *}$ Xiang-Shan Wang ${ }^{\dagger, *}$

† School of Chemistry and Materials Science, Jiangsu Normal University, Xuzhou, Jiangsu 221116, China

¥ Department of Chemistry, KTH Royal Institute of Technology, SE-100 44 Stockholm, Sweden

* E-mail: liujq316@jsnu.edu.cn (J.-Q.L.); karkas@kth.se (M.D.K.); xswang1974@yahoo.com (X.-S.W.) 


\section{Table of Contents}

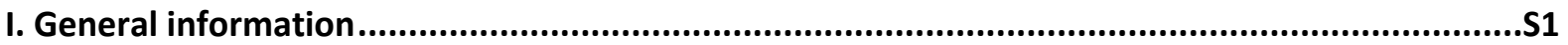

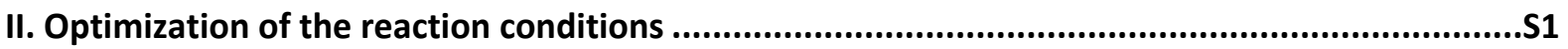

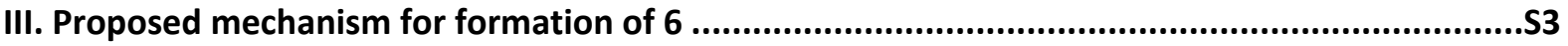

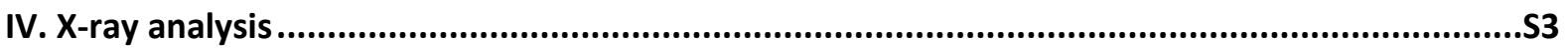

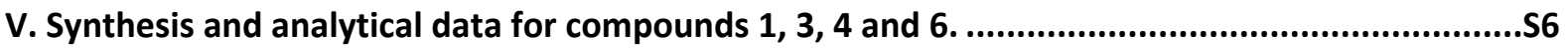

Typical synthetic procedure for compounds $\mathbf{1}$ (with 1a as an example)........................................S6

Typical synthetic procedure for compounds $\mathbf{3}$ (with $\mathbf{3 a}$ as an example).......................................S6

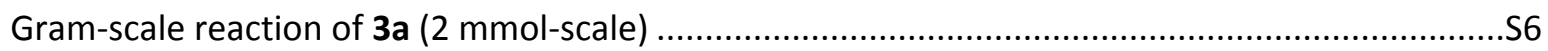

Typical synthetic procedure for compounds 4 (with 4 a as an example) ........................................S7

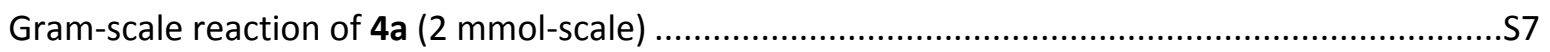

Typical synthetic procedure for compound $\mathbf{6}$ (with $\mathbf{6 a}$ as an example) …......................................

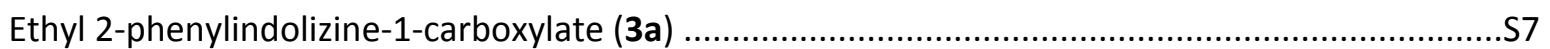

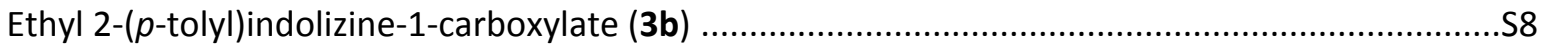

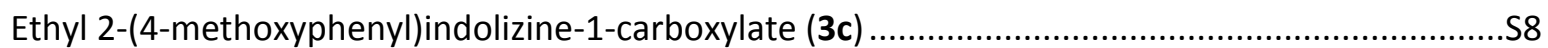

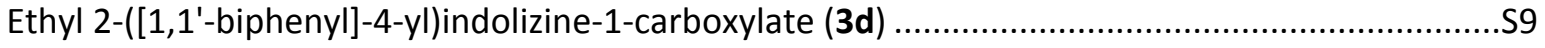

Ethyl 2-(4-(trifluoromethyl)phenyl)indolizine-1-carboxylate (3e) ..............................................S9

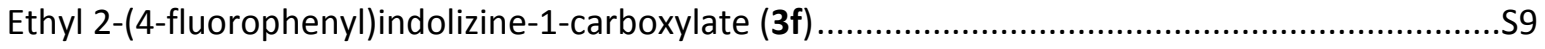

Ethyl 2-(4-chlorophenyl)indolizine-1-carboxylate (3g) .............................................................

Ethyl 2-(4-bromophenyl)indolizine-1-carboxylate (3h) ...............................................................

Ethyl 2-(4-cyanophenyl)indolizine-1-carboxylate (3i) .................................................................

Ethyl 2-(4-nitrophenyl)indolizine-1-carboxylate (3j) ...................................................................

Methyl 2-phenylindolizine-1-carboxylate (3k) ..................................................................... 11

Methyl 2-(p-tolyl)indolizine-1-carboxylate (3I) …........................................................................

Methyl 2-([1,1'-biphenyl]-4-yl)indolizine-1-carboxylate (3m) ....................................................S12

Methyl 2-(4-nitrophenyl)indolizine-1-carboxylate (3n) ….........................................................S12

Methyl 2-(4-bromophenyl)indolizine-1-carboxylate (30) ..........................................................S13

Ethyl 2-(3-chlorophenyl)indolizine-1-carboxylate (3p) …..........................................................S13

Ethyl 2-(3-nitrophenyl)indolizine-1-carboxylate (3q) ................................................................ 13

Ethyl 2-(3,4-dichlorophenyl)indolizine-1-carboxylate (3r) .......................................................... 14 
Methyl 2-(3,4-dichlorophenyl)indolizine-1-carboxylate (3s)

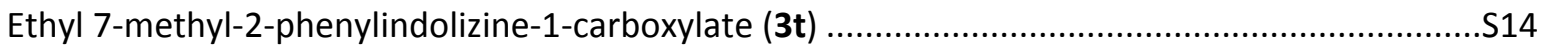

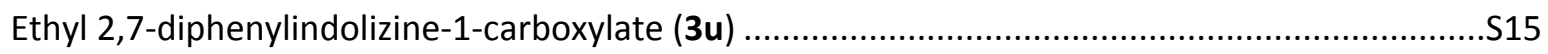

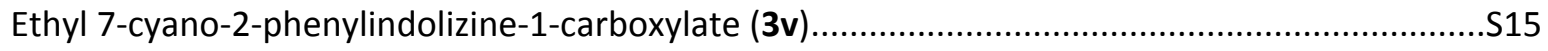

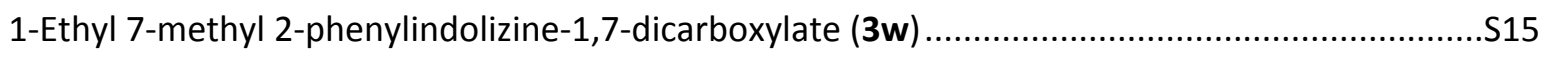

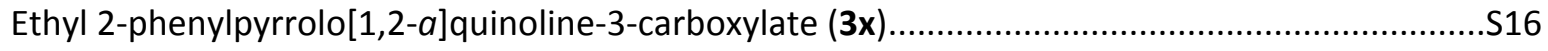

Methyl 2-phenylpyrrolo[1,2-a]quinoline-3-carboxylate (3y) .............................................S16

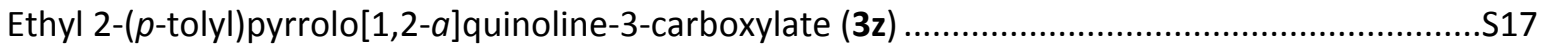

Ethyl 2-(4-methoxyphenyl)pyrrolo[1,2-a]quinoline-3-carboxylate (3aa)................................S17

Ethyl 2-(4-bromophenyl)pyrrolo[1,2-a]quinoline-3-carboxylate (3ab) .................................S17

Ethyl 2-(4-nitrophenyl)pyrrolo[1,2-a]quinoline-3-carboxylate (3ac) ....................................S18

Ethyl 2-(3-chlorophenyl)pyrrolo[1,2-a]quinoline-3-carboxylate (3ad) ..................................S18

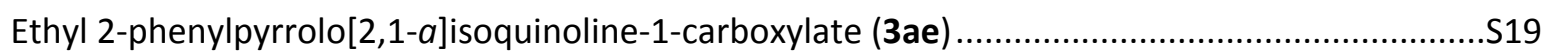

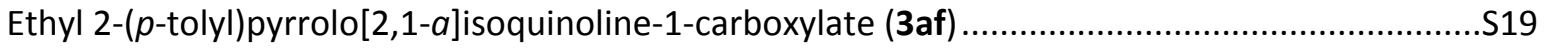

Ethyl 2-(4-methoxyphenyl)pyrrolo[2,1-a]isoquinoline-1-carboxylate (3ag) ..............................S19

Ethyl 2-(4-nitrophenyl)pyrrolo[2,1-a]isoquinoline-1-carboxylate (3ah) ...............................S20

Ethyl 2-(3-chlorophenyl)pyrrolo[2,1-a]isoquinoline-1-carboxylate (3ai) ................................S20

Ethyl-2-(3,4-dichlorophenyl)pyrrolo[2,1-a]isoquinoline-1-carboxylate (3aj) ...........................S21

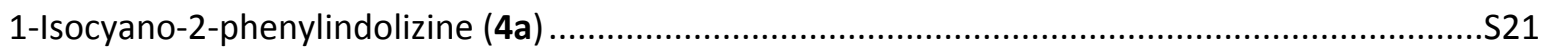

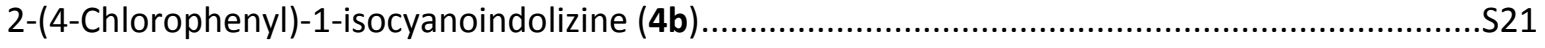

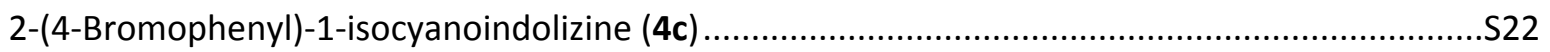

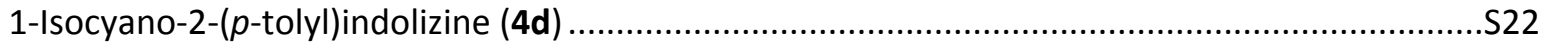

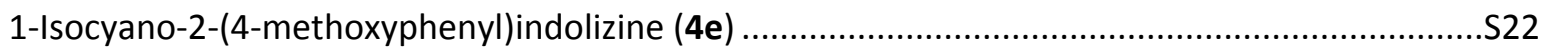

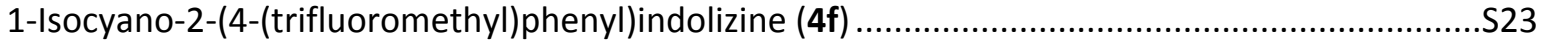

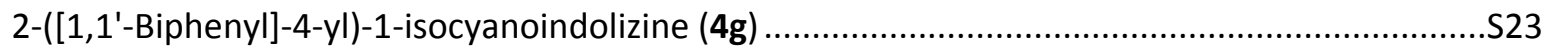

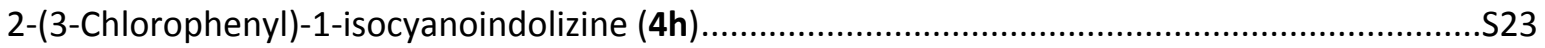

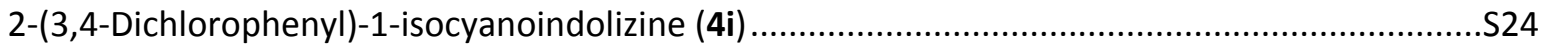

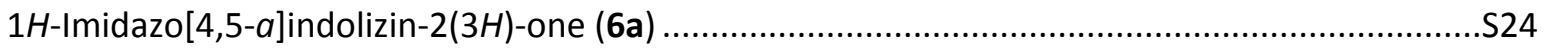

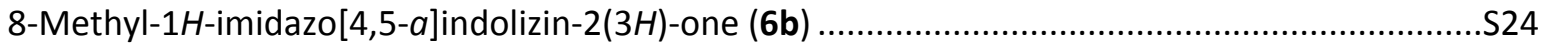

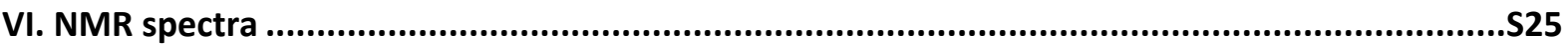


Ethyl 2-phenylindolizine-1-carboxylate (3a)

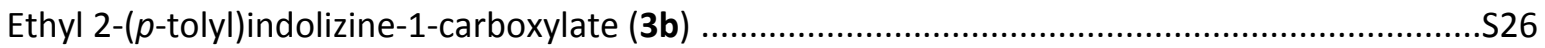

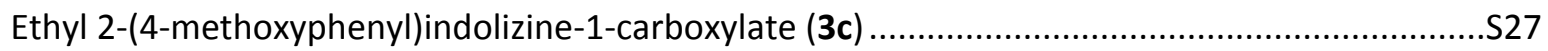

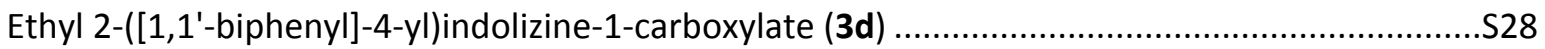

Ethyl 2-(4-(trifluoromethyl)phenyl)indolizine-1-carboxylate (3e) ........................................S29

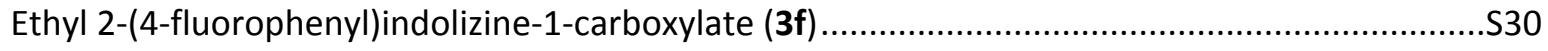

Ethyl 2-(4-chlorophenyl)indolizine-1-carboxylate (3g) ...................................................S31

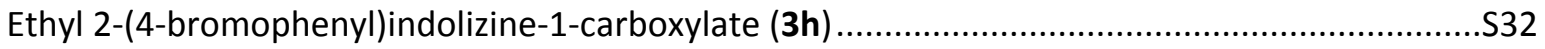

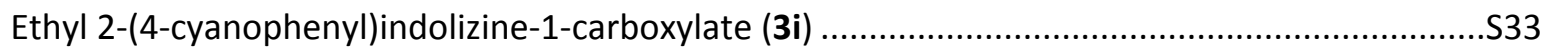

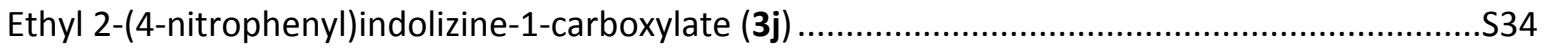

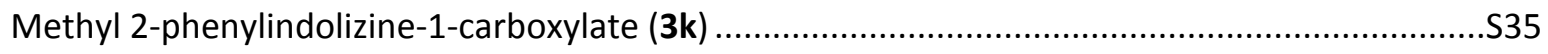

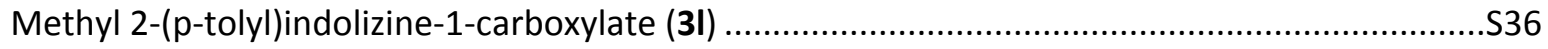

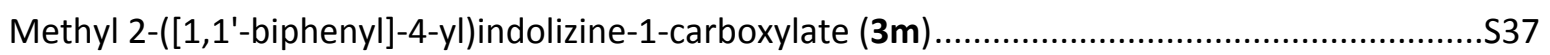

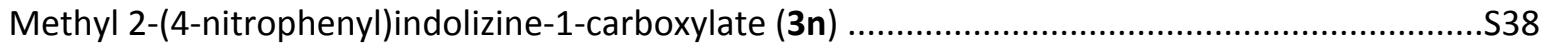

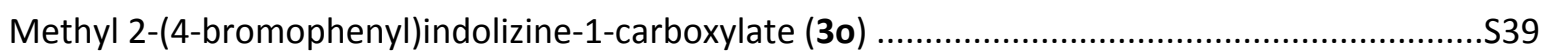

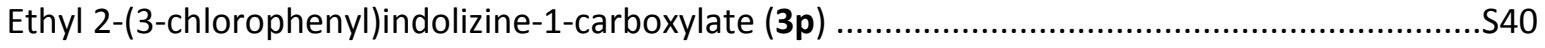

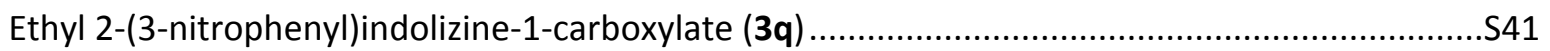

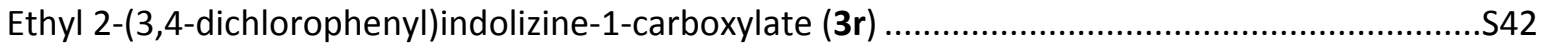

Methyl 2-(3,4-dichlorophenyl)indolizine-1-carboxylate (3s) ..............................................S43

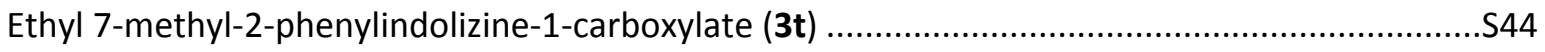

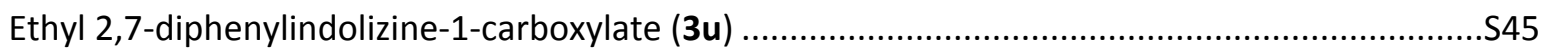

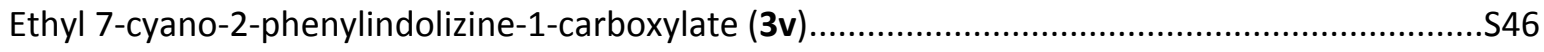

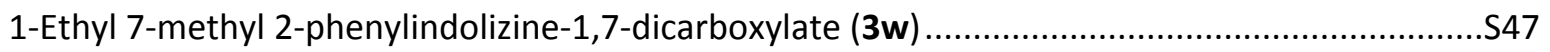

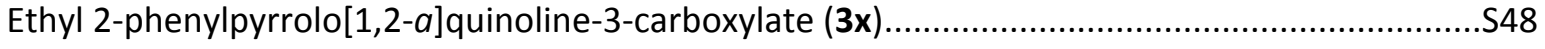

Methyl 2-phenylpyrrolo[1,2-a]quinoline-3-carboxylate (3y) ............................................S49

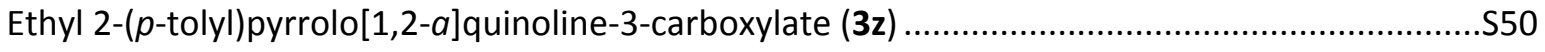

Ethyl 2-(4-methoxyphenyl)pyrrolo[1,2-a]quinoline-3-carboxylate (3aa)................................S51

Ethyl 2-(4-bromophenyl)pyrrolo[1,2-a]quinoline-3-carboxylate (3ab) ................................S52

Ethyl 2-(4-nitrophenyl)pyrrolo[1,2-a]quinoline-3-carboxylate (3ac) ..................................S53

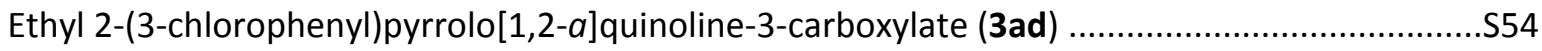


Ethyl 2-phenylpyrrolo[2,1-a]isoquinoline-1-carboxylate (3ae)

Ethyl 2-(p-tolyl)pyrrolo[2,1-a]isoquinoline-1-carboxylate (3af) . S56

Ethyl 2-(4-methoxyphenyl)pyrrolo[2,1-a]isoquinoline-1-carboxylate (3ag) .... S57

Ethyl 2-(4-nitrophenyl)pyrrolo[2,1-a]isoquinoline-1-carboxylate (3ah) S58

Ethyl 2-(3-chlorophenyl)pyrrolo[2,1-a]isoquinoline-1-carboxylate (3ai) S59

Ethyl-2-(3,4-dichlorophenyl)pyrrolo[2,1-a]isoquinoline-1-carboxylate (3aj). .560

1-Isocyano-2-phenylindolizine (4a) S61

2-(4-Chlorophenyl)-1-isocyanoindolizine (4b). S62

2-(4-Bromophenyl)-1-isocyanoindolizine (4c) .563

1-Isocyano-2-( $p$-tolyl)indolizine (4d) .564

1-Isocyano-2-(4-methoxyphenyl)indolizine (4e) .565

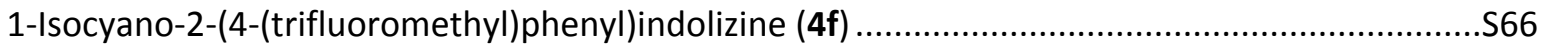

2-([1,1'-Biphenyl]-4-yl)-1-isocyanoindolizine (4g) .567

2-(3-Chlorophenyl)-1-isocyanoindolizine (4h). S68

2-(3,4-Dichlorophenyl)-1-isocyanoindolizine (4i) S69

$1 H$-Imidazo $[4,5-a]$ indolizin-2(3H)-one $(6 a)$ S70

8-Methyl-1H-imidazo[4,5-a]indolizin-2(3H)-one (6b) S71 


\section{General information}

All reagents were purchased from commercial suppliers and used without further treatment unless otherwise indicated. The products were purified by column chromatography over silica gel. ${ }^{1} \mathrm{H}$ NMR and ${ }^{13} \mathrm{C}$ NMR spectra were recorded at $25{ }^{\circ} \mathrm{C}$ on a Varian NMR spectrometer at $400 \mathrm{MHz}$ and 101 $\mathrm{MHz}$, respectively, with TMS as an internal standard. Mass spectra were recorded on BRUKER AutoflexIII Smartbeam MS-spectrometer. High resolution mass spectra (HRMS) were recorded on Brucker micrOTOF spectrometer with ESI ionisation method. Infrared (IR) spectra were recorded on a Bruker TENSOR 27 FT-IR spectrometer.

\section{Optimization of the reaction conditions}

Table S1. Conditions for the synthesis of disubstituted indolizines of $\mathbf{3 a}$ and $\mathbf{4 a}$. $^{\mathrm{a}}$

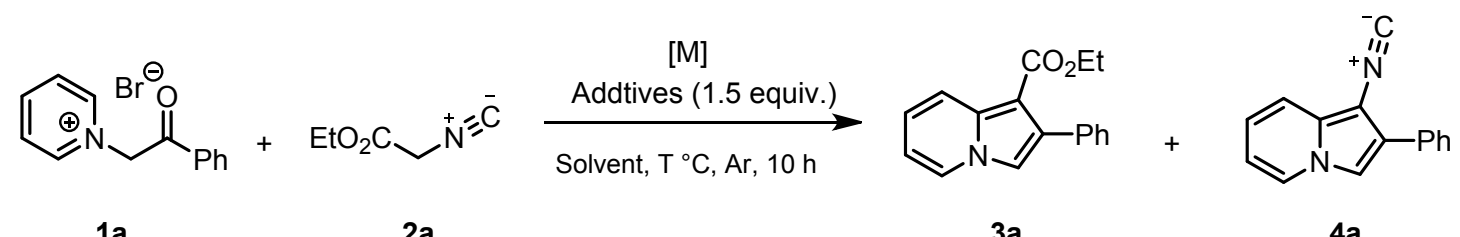

$1 a$

\begin{tabular}{|c|c|c|c|c|c|c|}
\hline \multirow[t]{2}{*}{ Entry } & \multirow[t]{2}{*}[\mathrm{Ag}]{} & \multirow[t]{2}{*}{ Additive } & \multirow[t]{2}{*}{ Solvent } & \multirow[t]{2}{*}{$\mathrm{T}\left({ }^{\circ} \mathrm{C}\right)$} & \multicolumn{2}{|c|}{ Yield (\%) } \\
\hline & & & & & $3 a$ & $4 a$ \\
\hline 1 & $\mathrm{Ag}_{2} \mathrm{CO}_{3}$ & - & 1,4-Dioxane & 25 & 39 & 14 \\
\hline 2 & $\mathrm{Ag}_{2} \mathrm{O}$ & - & 1,4-Dioxane & 25 & 22 & 12 \\
\hline 3 & $\mathrm{AgF}$ & - & 1,4-Dioxane & 25 & 29 & 8 \\
\hline 4 & AgOTf & - & 1,4-Dioxane & 25 & 24 & 8 \\
\hline 5 & $\mathrm{Ag}_{2} \mathrm{CO}_{3}$ & $\mathrm{~K}_{2} \mathrm{CO}_{3}$ & 1,4-Dioxane & 25 & 45 & 28 \\
\hline 6 & $\mathrm{Ag}_{2} \mathrm{CO}_{3}$ & $\mathrm{Cs}_{2} \mathrm{CO}_{3}$ & 1,4-Dioxane & 25 & 43 & 12 \\
\hline 7 & $\mathrm{Ag}_{2} \mathrm{CO}_{3}$ & $\mathrm{Et}_{3} \mathrm{~N}$ & 1,4-Dioxane & 25 & 89 & trace \\
\hline 8 & $\mathrm{Ag}_{2} \mathrm{CO}_{3}$ & $\mathrm{NaH}$ & 1,4-Dioxane & 25 & 38 & 12 \\
\hline 9 & $\mathrm{Ag}_{2} \mathrm{CO}_{3}$ & DBU & 1,4-Dioxane & 25 & trace & trace \\
\hline 10 & $\mathrm{Ag}_{2} \mathrm{CO}_{3}$ & $\mathrm{Et}_{3} \mathrm{~N}$ & DMF & 25 & 18 & 36 \\
\hline 11 & $\mathrm{Ag}_{2} \mathrm{CO}_{3}$ & $\mathrm{Et}_{3} \mathrm{~N}$ & THF & 25 & 35 & 16 \\
\hline 12 & $\mathrm{Ag}_{2} \mathrm{CO}_{3}$ & $\mathrm{Et}_{3} \mathrm{~N}$ & $\mathrm{CH}_{3} \mathrm{CN}$ & 25 & 56 & 15 \\
\hline 13 & $\mathrm{Ag}_{2} \mathrm{CO}_{3}$ & $\mathrm{Et}_{3} \mathrm{~N}$ & DCE & 25 & 47 & 18 \\
\hline 14 & $\mathrm{Ag}_{2} \mathrm{CO}_{3}$ & $\mathrm{Et}_{3} \mathrm{~N}$ & DMF & 50 & 42 & 56 \\
\hline 15 & $\mathrm{Ag}_{2} \mathrm{CO}_{3}$ & $\mathrm{Et}_{3} \mathrm{~N}$ & DMF & 80 & 39 & 47 \\
\hline $16^{c}$ & $\mathrm{Ag}_{2} \mathrm{CO}_{3}$ & $\mathrm{Et}_{3} \mathrm{~N}$ & DMF & 50 & $<5$ & 87 \\
\hline $17^{d}$ & $\mathrm{Ag}_{2} \mathrm{CO}_{3}$ & $\mathrm{Et}_{3} \mathrm{~N}$ & DMF & 50 & $<5$ & 76 \\
\hline $18^{e}$ & Cul & - & 1,4-dioxane & 25 & 0 & 0 \\
\hline $19^{f}$ & $\mathrm{Pd}(\mathrm{OAc})_{2}$ & - & 1,4-dioxane & 25 & 0 & 0 \\
\hline $20^{f}$ & $\mathrm{AuCl}\left(\mathrm{PPh}_{3}\right)_{2}$ & - & 1,4-dioxane & 25 & 0 & 0 \\
\hline
\end{tabular}




\begin{tabular}{ccccccc}
\hline $21^{g}$ & $\mathrm{Ag}_{2} \mathrm{CO}_{3}$ & $\mathrm{Et}_{3} \mathrm{~N}$ & 1,4-dioxane & 25 & 71 & 19 \\
$22^{h}$ & $\mathrm{Ag}_{2} \mathrm{CO}_{3}$ & $\mathrm{Et}_{3} \mathrm{~N}$ & 1,4-dioxane & 25 & 87 & tace \\
23 & - & $\mathrm{Et}_{3} \mathrm{~N}$ & 1,4-dioxane & 25 & 0 & 0 \\
24 & - & $\mathrm{Et}_{3} \mathrm{~N}$ & $\mathrm{DMF}$ & 50 & 0 & 0 \\
$25^{i}$ & $\mathrm{Ag}_{2} \mathrm{CO}_{3}$ & $\mathrm{Et}_{3} \mathrm{~N}$ & $\mathrm{DMF}$ & 50 & 62 & 21 \\
$26^{\mathrm{j}}$ & $\mathrm{Ag}_{2} \mathrm{CO}_{3}$ & $\mathrm{Et}_{3} \mathrm{~N}$ & $\mathrm{DMF}$ & 50 & trace & 16 \\
\hline
\end{tabular}

a Reaction conditions: 1a (139 mg, $0.5 \mathrm{mmol}, 1$ equiv.), 2a (66 $\mu \mathrm{L}, 0.6 \mathrm{mmol}, 1.2$ equiv.), [Ag] (1.5 equiv.), base (1.5 equiv.), solvent $(3.0 \mathrm{~mL}), 25{ }^{\circ} \mathrm{C}, 5 \mathrm{~h} .{ }^{b}$ Isolated yields. ${ }^{c} 30 \mathrm{~mol} \%$ of $\mathrm{Ag}_{2} \mathrm{CO}_{3} .{ }^{d} 50 \mathrm{~mol} \%$ of $\mathrm{Ag}_{2} \mathrm{CO}_{3} .{ }^{e}$ The amount of metal salts is $30 \mathrm{~mol} \% .{ }^{f}$ The amount of metal catalyst is $10 \mathrm{~mol} \%{ }^{g}$ The amount of metal salts is 1.0 equiv. ${ }^{h}$ The amount of metal salts is 2.0 equiv. ${ }^{i}$ The amount of metal salts is $20 \mathrm{~mol} \% .^{j}$ Under air condition. 


\section{Proposed mechanism for formation of 6}

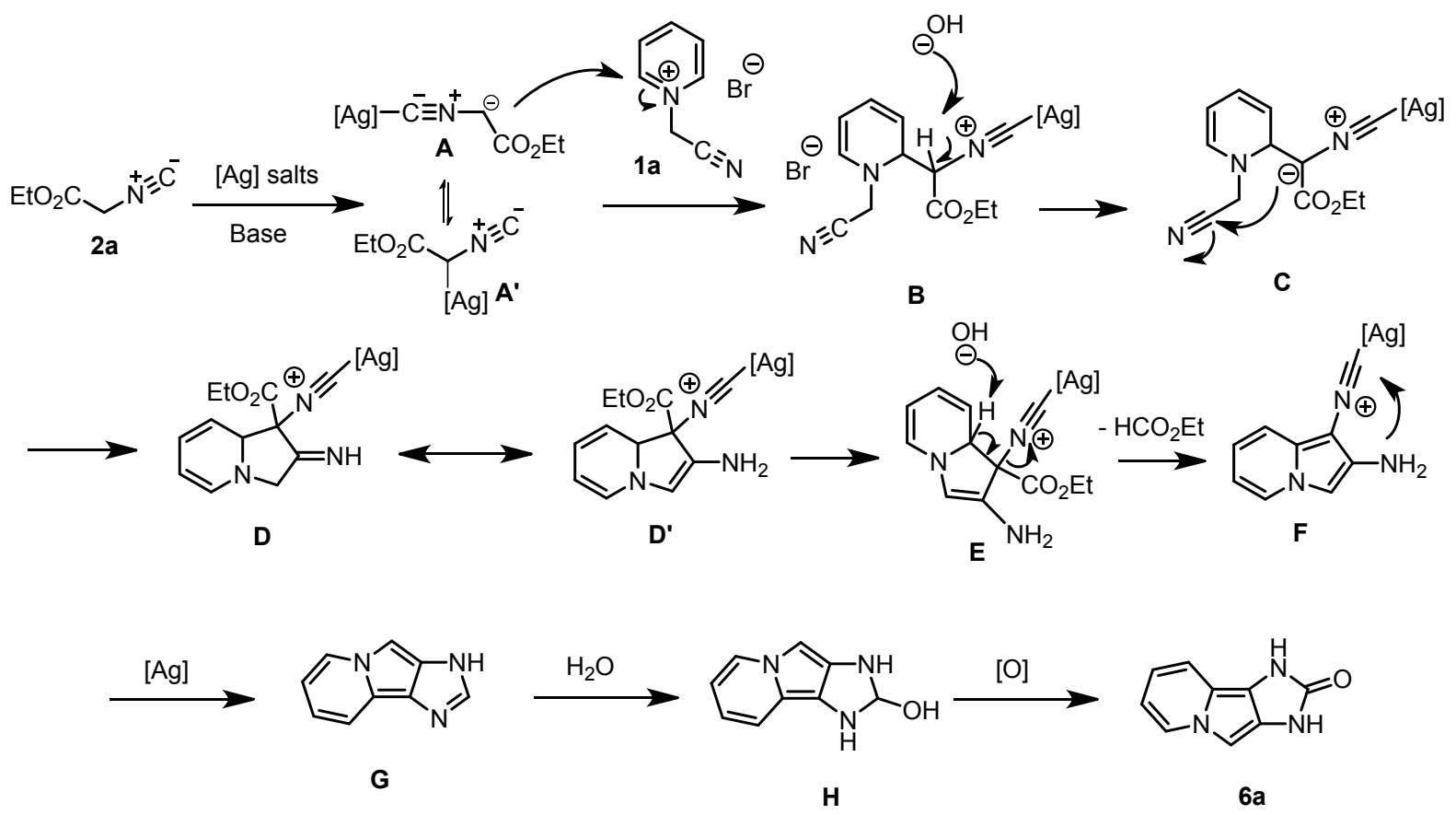

Figure S1. Proposed mechanism for formation of 6.

The formation of product $\mathbf{6}$ is similar to $\mathbf{4}$ from the raw material to intermediate $\mathbf{F}$, which is followed by intramolecular 1,1-addition, hydration to form intermediate $\mathbf{H}$. Intermediate $\mathbf{H}$ is an unstable intermediate, which is quickly oxidized into the final product 6 under air.1,2,3

\section{X-ray analysis}

Compound $3 \mathrm{c}(83 \mathrm{mg})$ and 3 ae $(98 \mathrm{mg})$ was dissolved in a centrifuge tube with $100 \mu \mathrm{L}$ petroleum etherand $50 \mu \mathrm{L}$ ethyl acetate, respectively. Two days later, we can get the the single-crystal of $\mathbf{3 c}$ and 3ae. The structures of the $N$-fused heterocyclic scaffolds were further confirmed by single crystal X-ray diffraction analysis. The X-ray diffraction data for the reported compounds was recorded at 296(2) K on Oxford Diffraction Gemini R Ultra diffractometer using $\omega$ scan technique with Mo-Ka radiation $(\lambda=0.71073 \AA)$. The structures were solved by the Direct Method in SHELXS-97 and refined by full-matrix least-squares techniques in SHELXL-97. ${ }^{4}$ Non-hydrogen atoms were refined with anisotropic temperature parameters, and hydrogen atoms of the ligands were refined as rigid groups. Basic information pertaining to crystal parameters and structure refinement is summarized in Tables S2 and S3. 
Table S2. Crystal structure and refinement data for compound 3c (thermal ellipsoids at $30 \%$ probability).
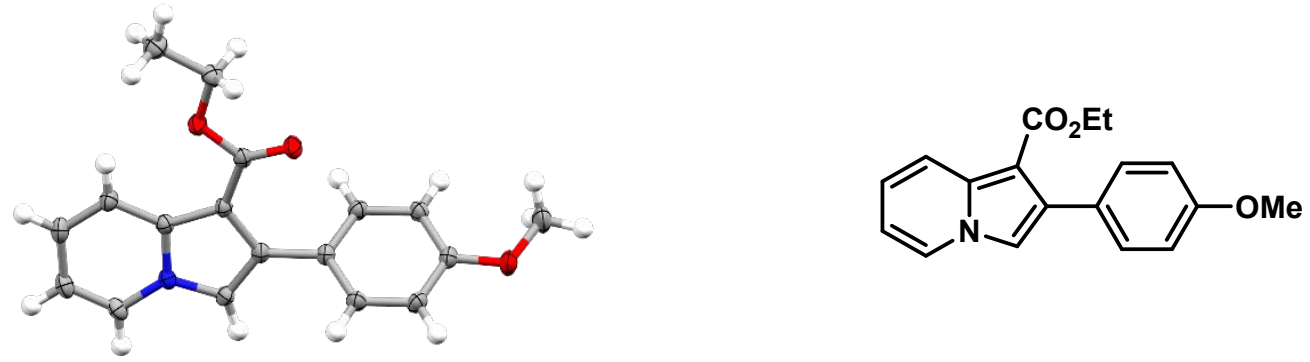

\begin{tabular}{|l|l|}
\hline Empirical formula & $\mathrm{C}_{18} \mathrm{H}_{17} \mathrm{NO}_{3}$ \\
\hline Temperature & $296(2) \mathrm{K}$ \\
\hline Wavelength & $0.71073 \AA$ \\
\hline Space group & $\mathrm{P} 2(1) / \mathrm{c}$ \\
\hline Unit cell dimensions & $\mathrm{a}=5.9127(13) \AA$ \\
& $\mathrm{b}=17.019(4) \AA$ \\
$\mathrm{C}=14.845(3) \AA$ \\
& $\mathrm{alpha}=90.00 \mathrm{deg}$. \\
& $\mathrm{beta}=97.034(3) \mathrm{deg}$. \\
\hline gamma $=90.00 \mathrm{deg}$.
\end{tabular}


Table S3. Crystal structure and refinement data for compound 3ae (thermal ellipsoids at 30\% probability).
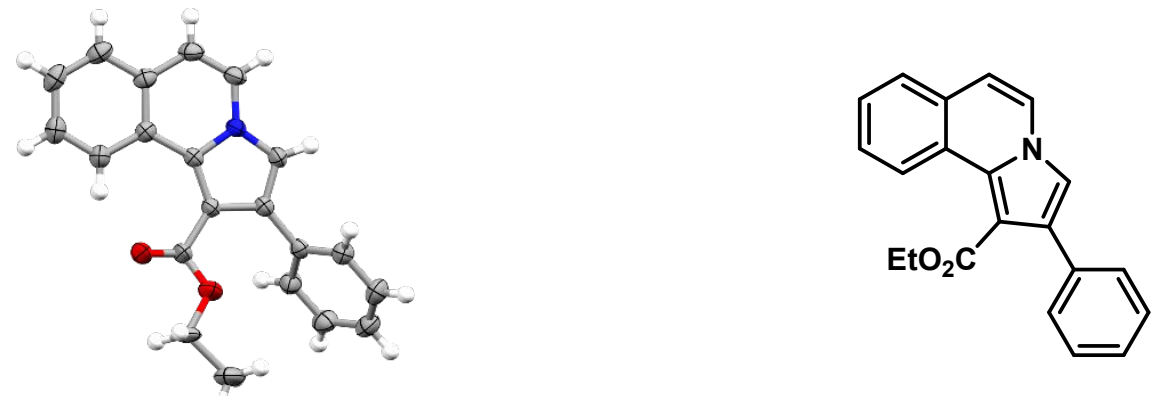

\begin{tabular}{|c|c|}
\hline Empirical formula & $\mathrm{C}_{21} \mathrm{H}_{17} \mathrm{NO}_{2}$ \\
\hline Temperature & $296(2) \mathrm{K}$ \\
\hline Wavelength & $0.71073 \AA$ \\
\hline Space group & $\mathrm{P} 2(1) / \mathrm{n}$ \\
\hline \multirow[t]{6}{*}{ Unit cell dimensions } & $a=11.574(3) \AA$ \\
\hline & $b=7.8232(18) \AA$ \\
\hline & $c=18.819(4) \AA$ \\
\hline & alpha = 90.00 deg. \\
\hline & beta $=102.098(3)$ deg. \\
\hline & gamma $=90.00 \mathrm{deg}$. \\
\hline Volume & $1666.2(7) \AA^{3}$ \\
\hline Z & 4 \\
\hline Calculated density & $1.257 \mathrm{Mg} / \mathrm{m}^{3}$ \\
\hline Absorption coefficient & $0.081 \mathrm{~mm}^{-1}$ \\
\hline$F(000)$ & 664 \\
\hline Crystal size & $0.118 \times 0.113 \times 0.101 \mathrm{~mm}$ \\
\hline Theta range for data collection & 2.83 to $25.01 \mathrm{deg}$. \\
\hline Reflections collected / unique & $8137 / 2927[\mathrm{R}(\mathrm{int})=0.0213]$ \\
\hline Data / restraints / parameters & $2927 / 0 / 246$ \\
\hline Goodness-of-fit on $F^{2}$ & 1.028 \\
\hline Final R indices [I>2sigma(I)] & $\mathrm{R} 1=0.0482, \mathrm{wR} 2=0.1274$ \\
\hline R indices (all data) & $\mathrm{R} 1=0.0600, \mathrm{wR} 2=0.1375$ \\
\hline
\end{tabular}




\section{Synthesis and analytical data for compounds $1,3,4$ and 6.}

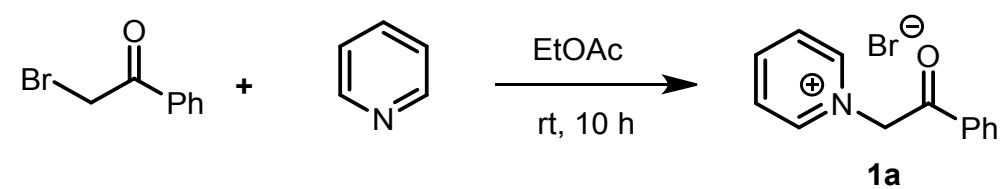

Typical synthetic procedure for compounds 1 (with 1a as an example): In a $100 \mathrm{~mL}$ beaker equipped with a magnetic stir bar was add $\alpha$-bromoacetophenone $(2.090 \mathrm{~g}, 10.5 \mathrm{mmol})$, pyridine $(800 \mu \mathrm{L}, 10$ $\mathrm{mmol}$ ) and $30 \mathrm{~mL}$ ethyl acetate. The reaction mixture was stirred at room temperature for about $10 \mathrm{~h}$. After the reaction is basically completed, the solution is filtered under reduced pressure with a Buchner funnel, the filter cake is washed with ethyl acetate, the filter cake is collected, and dried to obtain the raw material $N$-phenacyl bromopyridine $1 a^{5,6}$

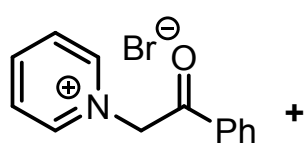

1a

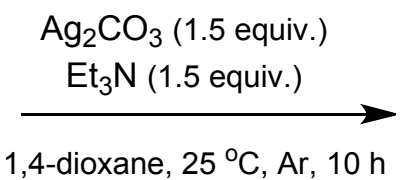

$2 \mathrm{a}$<smiles>CCOC(=O)c1c(-c2ccccc2)cn2ccccc12</smiles>

$3 a$

Typical synthetic procedure for compounds 3 (with 3a as an example): To a $25 \mathrm{~mL}$ Schlenk tube equipped with a magnetic stir bar was added 1-(2-oxo-2-phenylethyl)pyridin-1-ium bromide 1a (195 $\mathrm{mg}, 0.50 \mathrm{mmol})$, ethyl 2-isocyanoacetate $2 \mathrm{a}(66 \mu \mathrm{L}, 0.60 \mathrm{mmol}), 1,4$-dioxane $(3.0 \mathrm{~mL})$, and $\mathrm{Ag}_{2} \mathrm{CO}_{3}$ (206 mg, $0.75 \mathrm{mmol})$. The reaction mixture was stirred under Ar at $25^{\circ} \mathrm{C}$ in oil bath for about $10 \mathrm{~h}$. The resulting mixture was concentrated and the residue was taken up in $\mathrm{CH}_{2} \mathrm{Cl}_{2}$. The organic layer was washed with brine, dried over $\mathrm{MgSO}_{4}$ and concentrated. Purification of the crude product by column chromatography (silica gel; petroleum ether/ethyl acetate 15:1) afforded 3a as a white solid (118 mg, 89\% yield).

Gram-scale reaction of $\mathbf{3 a}$ ( $\mathbf{2} \mathbf{m m o l}-\mathrm{scale})$ : The experimental operation is the same as the model reaction to give target product 3a in $81 \%$ yield $(429 \mathrm{mg})$. 


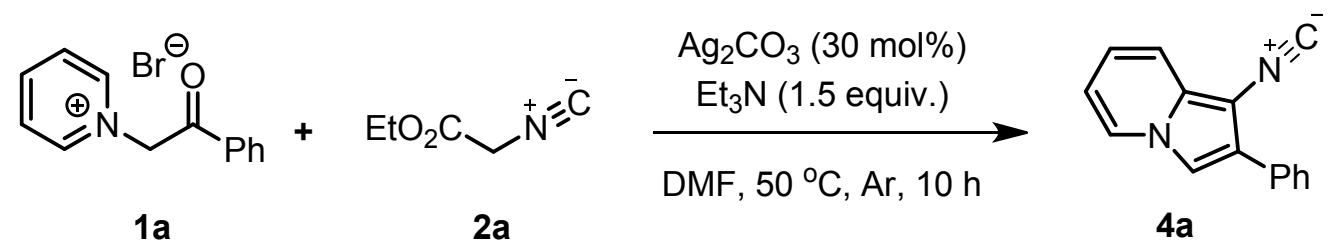

Typical synthetic procedure for compounds 4 (with 4 a as an example): To a $25 \mathrm{~mL}$ Schlenk tube equipped with a magnetic stir bar was added 1-(2-oxo-2-phenylethyl)pyridin-1-ium bromide 1a (195 $\mathrm{mg}, 0.50 \mathrm{mmol})$, ethyl 2-isocyanoacetate $2 \mathrm{a}(66 \mu \mathrm{L}, 0.60 \mathrm{mmol})$, DMF $(3.0 \mathrm{~mL})$, and $\mathrm{Ag}_{2} \mathrm{CO}_{3}(41 \mathrm{mg}$, $0.15 \mathrm{mmol})$. The resulting mixture was stirred under $\mathrm{Ar}$ at $50{ }^{\circ} \mathrm{C}$ in oil bath for about $10 \mathrm{~h}$. The resulting mixture was concentrated and the residue was taken up in $\mathrm{CH}_{2} \mathrm{Cl}_{2}$. The organic layer was washed with brine, dried over $\mathrm{MgSO}_{4}$ and concentrated. Purification of the crude product by column chromatography (silica gel; petroleum ether/ethyl acetate 15:1) afforded 4a as a white solid (95 mg, $87 \%$ yield).

Gram-scale reaction of $4 a(2 \mathrm{mmol}-\mathrm{scale})$ : the experimental operation is the same as the model reaction to give target product $4 \mathrm{a}$ in $62 \%$ yield $(270 \mathrm{mg})$.

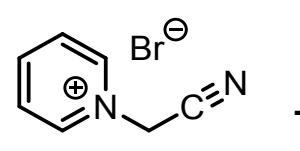

$5 a$<smiles>C#[N+]CCOCC</smiles>

2a

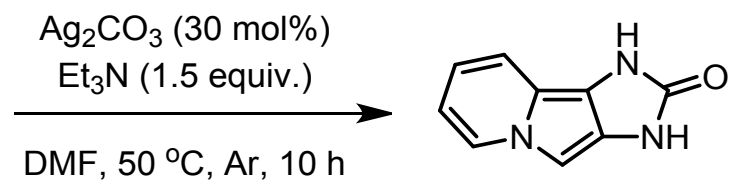

6a

Typical synthetic procedure for compound 6 (with 6 a as an example): To a $25 \mathrm{~mL}$ Schlenk tube equipped with a magnetic stir bar was added 1-(cyanomethyl)pyridin-1-ium bromide $5 a$ ( $99 \mathrm{mg}, 0.50$ $\mathrm{mmol})$, ethyl 2-isocyanoacetate $2 \mathrm{a}(66 \mu \mathrm{L}, 0.60 \mathrm{mmol}), \mathrm{DMF}(3.0 \mathrm{~mL})$, and $\mathrm{Ag}_{2} \mathrm{CO}_{3}(41 \mathrm{mg}, 0.15 \mathrm{mmol})$. The resulting mixture was stirred under $\mathrm{Ar}$ at $50{ }^{\circ} \mathrm{C}$ in oil bath for about $10 \mathrm{~h}$. The resulting mixture was concentrated and the residue was taken up in $\mathrm{CH}_{2} \mathrm{Cl}_{2}$. The organic layer was washed with brine, dried over $\mathrm{MgSO}_{4}$ and concentrated. Purification of the crude product by column chromatography (silica gel; petroleum ether/ethyl acetate 10:1) afforded 6 a as a white solid (71 mg, $79 \%$ yield).

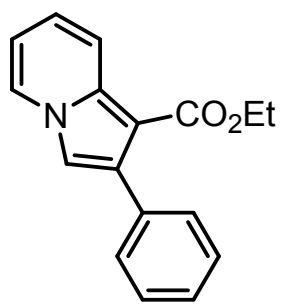

Ethyl 2-phenylindolizine-1-carboxylate (3a): Purification of the crude product by column chromatography (silica gel; petroleum ether/ethyl acetate 15:1); afforded $3 a$ as a white solid (118 $\mathrm{mg}, 89 \%$ yield); white solid, $\mathrm{mp} 117-118{ }^{\circ} \mathrm{C} ;{ }^{1} \mathrm{H}$ 
NMR $\left(400 \mathrm{MHz}, \mathrm{CDCl}_{3}\right): \delta_{\mathrm{H}} 8.24(\mathrm{~d}, J=9.2 \mathrm{~Hz}, 1 \mathrm{H}), 7.98(\mathrm{~d}, J=6.8 \mathrm{~Hz}, 1 \mathrm{H}), 7.53-7.50(\mathrm{~m}, 2 \mathrm{H}), 7.41-$ $7.33(\mathrm{~m}, 3 \mathrm{H}), 7.25(\mathrm{~s}, 1 \mathrm{H}), 7.09-7.04(\mathrm{~m}, 1 \mathrm{H}), 6.74-6.71(\mathrm{~m}, 1 \mathrm{H}), 4.25(\mathrm{q}, J=7.2 \mathrm{~Hz}, 2 \mathrm{H}), 1.23(\mathrm{t}, J=7.2$ $\mathrm{Hz}, 3 \mathrm{H}) ;{ }^{13} \mathrm{C}$ NMR $\left(101 \mathrm{MHz}, \mathrm{CDCl}_{3}\right): \delta_{\mathrm{C}} 165.0,136.7,134.9,132.7,129.9,127.5,127.0,125.5,122.4$, 120.3, 113.7, 112.6, 101.5, 59.3, 14.3; IR (KBr): v 2960, 2924, 2852, 1683, 1632, 1603, 1507, 1419, 1384, 1304, 1236, 1218, 1195, 1129, 1050, 788, 748, 700, $616 \mathrm{~cm}^{-1}$; HRMS (ESI-TOF, m/z): Calcd for $\mathrm{C}_{17} \mathrm{H}_{15} \mathrm{NNaO}_{2}[\mathrm{M}+\mathrm{Na}]^{+}:$288.0995, found 288.0989.

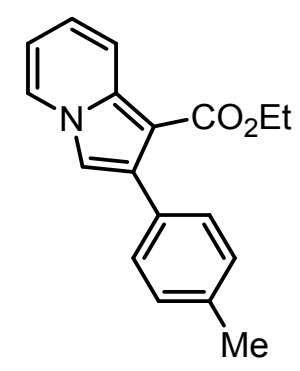

Ethyl 2-(p-tolyl)indolizine-1-carboxylate (3b): Purification of the crude product by column chromatography (silica gel; petroleum ether/ethyl acetate 15:1) afforded $\mathbf{3 b}$ as a white solid (113 mg, 81\% yield); mp $115-116^{\circ} \mathrm{C}$; ${ }^{1} \mathrm{H}$ NMR (400 $\left.\mathrm{MHz}, \mathrm{CDCl}_{3}\right): \delta_{\mathrm{H}} 8.22(\mathrm{~d}, J=9.2 \mathrm{~Hz}, 1 \mathrm{H}), 7.96(\mathrm{~d}, J=6.8 \mathrm{~Hz}, 1 \mathrm{H}), 7.41(\mathrm{~d}, J=8.0 \mathrm{~Hz}$, $2 \mathrm{H}), 7.22(\mathrm{~s}, 1 \mathrm{H}), 7.20(\mathrm{~d}, J=8.0 \mathrm{~Hz}, 2 \mathrm{H}), 7.07-7.03(\mathrm{~m}, 1 \mathrm{H}), 6.72-6.69(\mathrm{~m}, 1 \mathrm{H})$, $4.27(\mathrm{q}, J=7.2 \mathrm{~Hz}, 2 \mathrm{H}), 2.39(\mathrm{~s}, 3 \mathrm{H}), 1.26(\mathrm{t}, J=7.2 \mathrm{~Hz}, 3 \mathrm{H}) ;{ }^{13} \mathrm{C} N \mathrm{NMR}\left(101 \mathrm{MHz}, \mathrm{CDCl}_{3}\right): \delta_{\mathrm{C}} 165.0$, $136.71,136.7,132.8,131.9,129.8,128.3,125.5,122.3,120.3,113.6,112.5,101.5,59.3,21.2,14.3 ;$ IR (KBr): $v 3094,2976,2930,2906,1686,1630,1507,1491,1426,1385,1311,1297,1264,1218,1150$, 1105, 1038, 1015, 819, 759, $675 \mathrm{~cm}^{-1}$; HRMS (ESI-TOF, $\mathrm{m} / \mathrm{z}$ ): Calcd for $\mathrm{C}_{18} \mathrm{H}_{17} \mathrm{NNaO}_{5}[\mathrm{M}+\mathrm{Na}]^{+}$: 302.1151, found 302.1158.

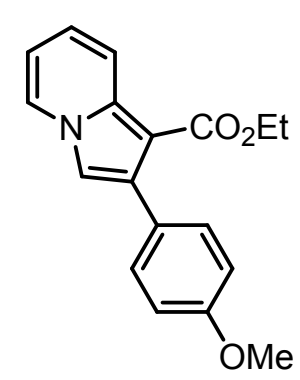

Ethyl 2-(4-methoxyphenyl)indolizine-1-carboxylate (3c): Purification of the crude product by column chromatography (silica gel; petroleum ether/ethyl acetate $12: 1$ ) afforded $3 \mathrm{c}$ as a white solid (123 $\mathrm{mg}, 83 \%$ yield); $\mathrm{mp} 146-147^{\circ} \mathrm{C}$; ${ }^{1} \mathrm{H} \mathrm{NMR}\left(400 \mathrm{MHz}, \mathrm{CDCl}_{3}\right): \delta_{\mathrm{H}} 8.22(\mathrm{~d}, J=8.8 \mathrm{~Hz}, 1 \mathrm{H}), 7.97(\mathrm{~d}, J=6.8 \mathrm{~Hz}, 1 \mathrm{H}), 7.46$ (d, $J=8.8 \mathrm{~Hz}, 2 \mathrm{H}), 7.22(\mathrm{~s}, 1 \mathrm{H}), 7.05\left(\mathrm{dd}, J=6.8 \mathrm{~Hz}, J^{\prime}=8.8 \mathrm{~Hz}, 1 \mathrm{H}\right), 6.94(\mathrm{~d}, J=8.4$ $\mathrm{Hz}, 2 \mathrm{H}), 6.73-6.69(\mathrm{~m}, 1 \mathrm{H}), 4.27(\mathrm{q}, J=7.2 \mathrm{~Hz}, 2 \mathrm{H}), 3.85(\mathrm{~s}, 3 \mathrm{H}), 1.27(\mathrm{t}, J=7.2 \mathrm{~Hz}, 3 \mathrm{H}) ;{ }^{13} \mathrm{C}$ NMR $(101$ $\left.\mathrm{MHz}, \mathrm{CDCl}_{3}\right): \delta_{\mathrm{C}} 165.0,158.9,136.7,132.5,131.0,127.3,125.4,122.3,120.3,113.5,113.1,112.5$ 101.5, 59.3, 55.3, 14.4; IR (KBr): v3008, 2963, 2908, 1685, 1610, 1506, 1490, 1430, 1406, 1389, 1309, 1245, 1220, 1179, 1128, 1109, 1040, 839, 786, 760, $676 \mathrm{~cm}^{-1}$; HRMS (ESI-TOF, m/z): Calcd for $\mathrm{C}_{18} \mathrm{H}_{17} \mathrm{NNaO}_{3}[\mathrm{M}+\mathrm{Na}]^{+}:$318.1101, found 318.1112. 


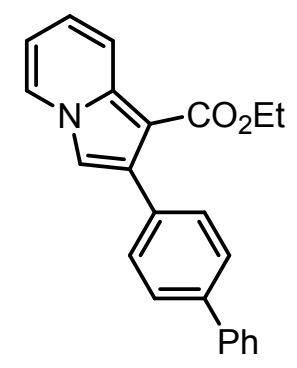

Ethyl 2-([1,1'-biphenyl]-4-yl)indolizine-1-carboxylate (3d): Purification of the crude product by column chromatography (silica gel; petroleum ether/ethyl acetate $15: 1$ ) afforded $\mathbf{3 d}$ as a white solid (133 $\mathrm{mg}, 78 \%$ yield); $\mathrm{mp} 197-198{ }^{\circ} \mathrm{C} ;{ }^{1} \mathrm{H}$ NMR $\left(400 \mathrm{MHz}, \mathrm{CDCl}_{3}\right): \delta_{\mathrm{H}} 8.25(\mathrm{~d}, J=9.2 \mathrm{~Hz}, 1 \mathrm{H}), 7.99(\mathrm{~d}, J=6.8 \mathrm{~Hz}, 1 \mathrm{H}), 7.66-$ $7.59(\mathrm{~m}, 6 \mathrm{H}), 7.47-7.44(\mathrm{~m}, 2 \mathrm{H}), 7.37-7.33(\mathrm{~m}, 1 \mathrm{H}), 7.29(\mathrm{~s}, 1 \mathrm{H}), 7.09-7.05(\mathrm{~m}$, 1H), 6.75-6.71 (m, 1H), $4.29(\mathrm{q}, J=7.2 \mathrm{~Hz}, 2 \mathrm{H}), 1.27(\mathrm{t}, J=7.2 \mathrm{~Hz}, 3 \mathrm{H}) ;{ }^{13} \mathrm{C} \mathrm{NMR}\left(101 \mathrm{MHz}, \mathrm{CDCl}_{3}\right): \delta_{\mathrm{C}}$ 165.0, 141.1, 139.9, 136.8, 133.9, 132.4, 130.3, 128.7, 127.2, 127.1, 126.3, 125.5, 122.4, 120.4, 113.7, 112.7, 101.6, 59.4, 14.3; IR (KBr): v 3092, 2976, 2906, 1687, 1609, 1507, 1490, 1427, 1388, 1309, $1246,1218,1167,1108,1040,831,759,708,675 \mathrm{~cm}^{-1}$; HRMS (ESI-TOF, $\mathrm{m} / \mathrm{z}$ ): Calcd for $\mathrm{C}_{23} \mathrm{H}_{19} \mathrm{NNaO}_{2}$ $[\mathrm{M}+\mathrm{Na}]^{+}: 364.1308$, found 364.1321 .

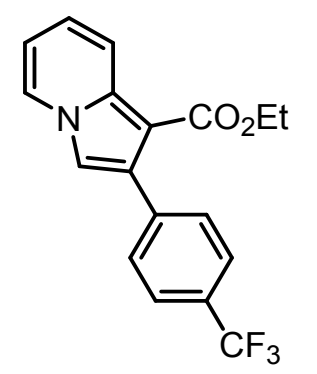

Ethyl 2-(4-(trifluoromethyl)phenyl)indolizine-1-carboxylate (3e): Purification of the crude product by column chromatography (silica gel; petroleum ether/ethyl acetate $15: 1$ ) afforded $3 \mathbf{e}$ as a white solid (125 mg, 75\% yield); mp $112-113{ }^{\circ} \mathrm{C}$; ${ }^{1} \mathrm{H}$ NMR $\left(400 \mathrm{MHz}, \mathrm{CDCl}_{3}\right): \delta_{\mathrm{H}} 8.25(\mathrm{~d}, J=9.2 \mathrm{~Hz}, 1 \mathrm{H}), 8.00(\mathrm{~d}, J=6.8 \mathrm{~Hz}, 1 \mathrm{H})$, 7.66-7.61 (m, 4H), $7.27(\mathrm{~s}, 1 \mathrm{H}), 7.12-7.08(\mathrm{~m}, 1 \mathrm{H}), 6.78-6.75(\mathrm{~m}, 1 \mathrm{H}), 4.26(\mathrm{q}, J=$ $7.2 \mathrm{~Hz}, 2 \mathrm{H}), 1.24(\mathrm{t}, J=7.2 \mathrm{~Hz}, 3 \mathrm{H}) ;{ }^{13} \mathrm{C}$ NMR $\left(101 \mathrm{MHz}, \mathrm{CDCl}_{3}\right): \delta_{\mathrm{C}} 164.7,138.8,136.8,131.3,130.2$, $129.1\left(q, J_{C-F}=32.1 \mathrm{~Hz}\right), 125.6,124.5\left(q, J_{C-F}=3.7 \mathrm{~Hz}\right), 124.4\left(q, J_{C-F}=270.5 \mathrm{~Hz}\right), 122.9,120.3,113.8$, $112.9,101.5,59.5,14.3 ; \mathrm{IR}(\mathrm{KBr}): v 3050,2993,2917,1678,1615,1552,1506,1490,1433,1388$, 1326, 1282, 1225, 1171, 1108, 1068, 1035, 784, 744, $690 \mathrm{~cm}^{-1}$; HRMS (ESI-TOF, $\mathrm{m} / \mathrm{z}$ ): Calcd for $\mathrm{C}_{18} \mathrm{H}_{14} \mathrm{~F}_{3} \mathrm{NNaO}_{2}[\mathrm{M}+\mathrm{Na}]^{+}:$356.0869, found 356.0869.

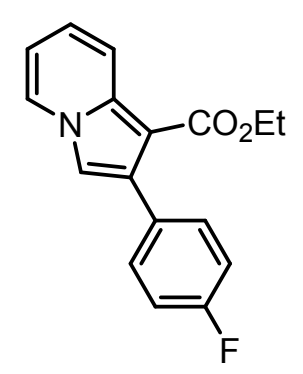

Ethyl 2-(4-fluorophenyl)indolizine-1-carboxylate (3f): Purification of the crude product by column chromatography (silica gel; petroleum ether/ethyl acetate 15:1) afforded $3 f$ as a white solid (122 $\mathrm{mg}, 86 \%$ yield); $\mathrm{mp} 126-127{ }^{\circ} \mathrm{C} ;{ }^{1} \mathrm{H}$ NMR $\left(400 \mathrm{MHz}, \mathrm{CDCl}_{3}\right): \delta_{\mathrm{H}} 8.23(\mathrm{~d}, J=8.8 \mathrm{~Hz}, 1 \mathrm{H}), 7.98(\mathrm{~d}, J=6.8 \mathrm{~Hz}, 1 \mathrm{H}), 7.49-7.54(\mathrm{~m}$, $2 \mathrm{H}), 7.22(\mathrm{~s}, 1 \mathrm{H}), 7.10-7.05(\mathrm{~m}, 3 \mathrm{H}), 6.75-6.72(\mathrm{~m}, 1 \mathrm{H}), 4.26(\mathrm{q}, J=7.2 \mathrm{~Hz}, 2 \mathrm{H})$, $1.25(\mathrm{t}, J=7.2 \mathrm{~Hz}, 3 \mathrm{H}) ;{ }^{13} \mathrm{C} \mathrm{NMR}\left(101 \mathrm{MHz}, \mathrm{CDCl}_{3}\right): \delta_{\mathrm{C}} 164.9,162.2\left(\mathrm{~d}, J_{C-F}=244.0 \mathrm{~Hz}\right), 136.7,131.7$, $131.5\left(d, J_{C-F}=7.9 \mathrm{~Hz}\right), 130.9\left(\mathrm{~d}, J_{C-F}=3.3 \mathrm{~Hz}\right), 125.5,122.6,120.4,114.4\left(\mathrm{~d}, J_{C-F}=21.2 \mathrm{~Hz}\right.$ ), 113.6, 112.7, 101.5, 59.4, 14.3; IR (KBr): v 3123, 2963, 2920, 1682, 1610, 1504, 1487, 1429, 1400, 1385, 
1307, 1262, 1218, 1156, 1130, 1111, 1034, 850, 798, 742, $670 \mathrm{~cm}^{-1}$; HRMS (ESI-TOF, $\mathrm{m} / \mathrm{z}$ ): Calcd for $\mathrm{C}_{17} \mathrm{H}_{14} \mathrm{FNNaO}_{2}[\mathrm{M}+\mathrm{Na}]^{+}:$306.0901, found 306.0908.

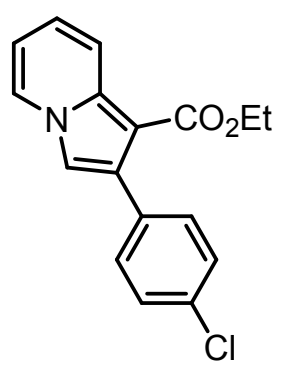

Ethyl 2-(4-chlorophenyl)indolizine-1-carboxylate (3g): Purification of the crude product by column chromatography (silica gel; petroleum ether/ethyl acetate 15:1) afforded $3 g$ as a white solid (136 mg, 96\% yield); mp $109-110{ }^{\circ} \mathrm{C} ;{ }^{1} \mathrm{H}$ NMR $\left(400 \mathrm{MHz}, \mathrm{CDCl}_{3}\right): \delta_{\mathrm{H}} 8.24(\mathrm{~d}, J=9.2 \mathrm{~Hz}, 1 \mathrm{H}), 7.99(\mathrm{~d}, J=7.2 \mathrm{~Hz}, 1 \mathrm{H}), 7.45(\mathrm{~d}, J=$ $8.4 \mathrm{~Hz}, 2 \mathrm{H}), 7.36(\mathrm{~d}, J=8.4 \mathrm{~Hz}, 2 \mathrm{H}), 7.24(\mathrm{~s}, 1 \mathrm{H}), 7.10-7.06(\mathrm{~m}, 1 \mathrm{H}), 6.76-6.73(\mathrm{~m}$, 1H), $4.27(\mathrm{q}, J=7.2 \mathrm{~Hz}, 2 \mathrm{H}), 1.26(\mathrm{t}, J=7.2 \mathrm{~Hz}, 3 \mathrm{H}) ;{ }^{13} \mathrm{C} \mathrm{NMR}\left(101 \mathrm{MHz}, \mathrm{CDCl}_{3}\right): \delta_{\mathrm{C}} 164.8,136.8,133.4$, 133.1, 131.5, 131.2, 1277, 125.5, 122.6, 120.4, 113.6, 112.8, 101.5, 59.4, 14.3; IR (KBr): v3124, 2972, $2918,1678,1607,1504,1482,1404,1383,1307,1278,1220,1154,1130,1111,1034,786,744,678$ $\mathrm{cm}^{-1}$; HRMS (ESI-TOF, $\mathrm{m} / \mathrm{z}$ ): Calcd for $\mathrm{C}_{17} \mathrm{H}_{14} \mathrm{ClNNaO}_{2}[\mathrm{M}+\mathrm{Na}]^{+}: 322.0605$, found 322.0608 .

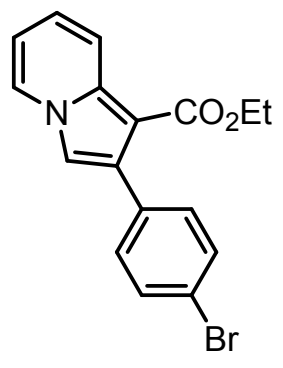

Ethyl 2-(4-bromophenyl)indolizine-1-carboxylate (3h): Purification of the crude product by column chromatography (silica gel; petroleum ether/ethyl acetate 15:1) afforded $3 \mathrm{~h}$ as a white solid (150 mg, 87\% yield); mp $124-125{ }^{\circ} \mathrm{C} ;{ }^{1} \mathrm{H}$ NMR $\left(400 \mathrm{MHz}, \mathrm{CDCl}_{3}\right): \delta_{\mathrm{H}} 8.23(\mathrm{~d}, J=9.2 \mathrm{~Hz}, 1 \mathrm{H}), 7.98(\mathrm{~d}, J=6.8 \mathrm{~Hz}, 1 \mathrm{H}), 7.51(\mathrm{~d}, J=$ $8.4 \mathrm{~Hz}, 2 \mathrm{H}), 7.38(\mathrm{~d}, J=8.4 \mathrm{~Hz}, 2 \mathrm{H}), 7.23(\mathrm{~s}, 1 \mathrm{H}), 7.08(\mathrm{dd}, J=9.2,6.8 \mathrm{~Hz}, 1 \mathrm{H})$, 6.76-6.72 (m, 1H), $4.27(\mathrm{q}, J=7.2 \mathrm{~Hz}, 2 \mathrm{H}), 1.26(\mathrm{t}, J=7.2 \mathrm{~Hz}, 3 \mathrm{H}) ;{ }^{13} \mathrm{C}$ NMR $\left(101 \mathrm{MHz}, \mathrm{CDCl}_{3}\right): \delta_{\mathrm{C}}$ $164.8,136.8,133.9,131.5,131.4,130.7,125.5,122.7,121.3,120.4,113.6,112.8,101.4,59.4,14.3 ;$ IR (KBr): v 3121, 2985, 2916, 1678, 1593, 1547, 1500, 1479, 1427, 1392, 1307, 1280, 1217, 1130, 1111, 1069, 1034, 1006, 783, 744, $667 \mathrm{~cm}^{-1}$; HRMS (ESI-TOF, $\left.\mathrm{m} / \mathrm{z}\right)$ : Calcd for $\mathrm{C}_{17} \mathrm{H}_{14} \mathrm{BrNNaO}_{2}[\mathrm{M}+\mathrm{Na}]^{+}$: 366.0100 , found 366.0105 .

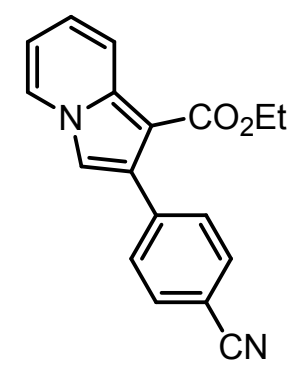

Ethyl 2-(4-cyanophenyl)indolizine-1-carboxylate (3i): Purification of the crude product by column chromatography (silica gel; petroleum ether/ethyl acetate 15:1) afforded $3 \mathbf{i}$ as a white solid (119 $\mathrm{mg}, 82 \%$ yield); $\mathrm{mp} 130-131{ }^{\circ} \mathrm{C} ;{ }^{1} \mathrm{H}$ NMR $\left(400 \mathrm{MHz}, \mathrm{CDCl}_{3}\right): \delta_{\mathrm{H}} 8.24(\mathrm{~d}, J=9.2 \mathrm{~Hz}, 1 \mathrm{H}), 8.00(\mathrm{~d}, J=6.8 \mathrm{~Hz}, 1 \mathrm{H}), 7.67(\mathrm{~d}, J=$ $8.0 \mathrm{~Hz}, 2 \mathrm{H}), 7.62(\mathrm{~d}, J=8.0 \mathrm{~Hz}, 2 \mathrm{H}), 7.28(\mathrm{~s}, 1 \mathrm{H}), 7.11(\mathrm{dd}, J=8.8,6.8 \mathrm{~Hz}, 1 \mathrm{H})$, 6.79-6.76 (m, 1H), $4.27(\mathrm{q}, J=7.2 \mathrm{~Hz}, 2 \mathrm{H}), 1.25(\mathrm{t}, J=7.2 \mathrm{~Hz}, 3 \mathrm{H}) ;{ }^{13} \mathrm{C} N \mathrm{NMR}\left(101 \mathrm{MHz}, \mathrm{CDCl}_{3}\right): \delta_{\mathrm{C}}$ 
164.6, 140.1, 137.0, 131.3, 130.8, 130.6, 125.6, 123.1, 120.5, 119.2, 113.9, 113.1, 110.6, 101.4, 59.6, 14.3; IR (KBr): v 3113, 2989, 2927, 2902, 2222, 1674, 1606, 1510, 1485, 1428, 1405, 1372, 1296, 1272, 1236, 1141, 1113, 1046, 785, 756, $671 \mathrm{~cm}^{-1}$; HRMS (ESI-TOF, $\left.m / z\right)$ : Calcd for $\mathrm{C}_{18} \mathrm{H}_{14} \mathrm{~N}_{2} \mathrm{NaO}_{2}[\mathrm{M}+$ $\mathrm{Na}]^{+}:$313.0947, found 313.0953.

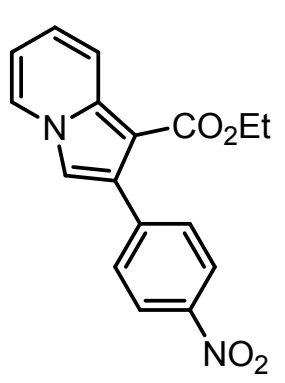

Ethyl 2-(4-nitrophenyl)indolizine-1-carboxylate (3j): Purification of the crude product by column chromatography (silica gel; petroleum ether/ethyl acetate 10:1) afforded $3 \mathbf{j}$ as a white solid (141 $\mathrm{mg}, 91 \%$ yield); $\mathrm{mp} 153-154{ }^{\circ} \mathrm{C} ;{ }^{1} \mathrm{H}$ NMR $\left.\left(400 \mathrm{MHz}^{\mathrm{CDCl}}\right)_{3}\right): \delta_{\mathrm{H}} 8.26-8.24(\mathrm{~m}, 3 \mathrm{H}), 8.02(\mathrm{~d}, J=7.2 \mathrm{~Hz}, 1 \mathrm{H}), 7.68(\mathrm{~d}, J=8.4 \mathrm{~Hz}$, 2H), $7.31(\mathrm{~s}, 1 \mathrm{H}), 7.12(\mathrm{dd}, J=9.2,6.8 \mathrm{~Hz}, 1 \mathrm{H}), 4.28(\mathrm{q}, J=7.2 \mathrm{~Hz}, 2 \mathrm{H}), 1.26(\mathrm{t}, J=$ $7.2 \mathrm{~Hz}, 3 \mathrm{H}) ;{ }^{13} \mathrm{C}$ NMR $\left(101 \mathrm{MHz}, \mathrm{CDCl}_{3}\right): \delta_{\mathrm{C}} 164.5,146.9,142.1,137.0,130.7,130.4,125.7,123.2$, $122.8,120.5,114.0,113.2,101.5,59.6,14.3 ; \mathrm{IR}(\mathrm{KBr}): v 3122,2983,2924,1683,1603,1515,1479$, $1422,1401,1340,1294,1274,1228,1131,1109,1049,1038,789,752,700,670 \mathrm{~cm}^{-1}$; HRMS (ESI-TOF, $m / z$ ): Calcd for $\mathrm{C}_{17} \mathrm{H}_{14} \mathrm{~N}_{2} \mathrm{NaO}_{2}[\mathrm{M}+\mathrm{Na}]^{+}:$333.0846, found 333.0852.

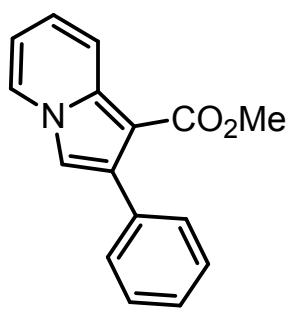

Methyl 2-phenylindolizine-1-carboxylate (3k): Purification of the crude product by column chromatography (silica gel; petroleum ether/ethyl acetate 15:1) afforded $3 \mathbf{k}$ as a white solid (111 mg, 88\% yield); mp 91-92 ${ }^{\circ} \mathrm{C} ;{ }^{1} \mathrm{H}$ NMR (400 $\left.\mathrm{MHz}, \mathrm{CDCl}_{3}\right): \delta_{\mathrm{H}} 8.23(\mathrm{~d}, J=9.2 \mathrm{~Hz}, 1 \mathrm{H}), 7.98(\mathrm{~d}, J=6.8 \mathrm{~Hz}, 1 \mathrm{H}), 7.51(\mathrm{~d}, J=7.2$ $\mathrm{Hz}, 2 \mathrm{H}), 7.42-7.38(\mathrm{~m}, 2 \mathrm{H}), 7.36-7.32(\mathrm{~m}, 1 \mathrm{H}), 7.25(\mathrm{~s}, 1 \mathrm{H}), 7.09-7.05(\mathrm{~m}, 1 \mathrm{H})$, $3.77(\mathrm{~s}, 3 \mathrm{H}) ;{ }^{13} \mathrm{C} \mathrm{NMR}\left(101 \mathrm{MHz}, \mathrm{CDCl}_{3}\right): \delta_{\mathrm{C}} 165.4,136.8,134.9,132.7,129.8,127.6,127.1,125.5$, $122.5,120.4,113.8,112.7,101.3,50.6$; IR (KBr): $v$ 3051, 2998, 2948, 2850, 1675, 1603, 1550, 1504, $1479,1450,1435,1405,1306,1222,1130,1113,1040,1021,790,755,702,667 \mathrm{~cm}^{-1}$; HRMS (ESI-TOF, $m / z)$ : Calcd for $\mathrm{C}_{16} \mathrm{H}_{13} \mathrm{NNaO}_{2}[\mathrm{M}+\mathrm{Na}]^{+}:$274.0838, found 274.0844 .

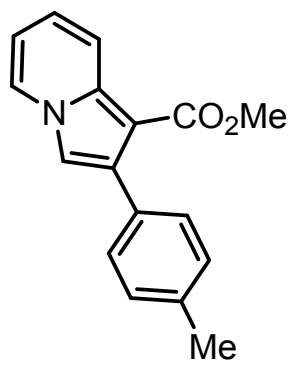

Methyl 2-(p-tolyl)indolizine-1-carboxylate (3l): Purification of the crude product by column chromatography (silica gel; petroleum ether/ethyl acetate 15:1) afforded $3 \mathrm{I}$ as a white solid (109 $\mathrm{mg}, 82 \%$ yield); mp $154-155^{\circ} \mathrm{C} ;{ }^{1} \mathrm{H}$ NMR (400 $\mathrm{MHz}, \mathrm{CDCl}_{3}$ ): $\delta_{\mathrm{H}} 8.22(\mathrm{~d}, J=9.2 \mathrm{~Hz}, 1 \mathrm{H}), 7.97(\mathrm{~d}, J=6.8 \mathrm{~Hz}, 1 \mathrm{H}), 7.41(\mathrm{~d}, J=8.0$ 
$\mathrm{Hz}, 2 \mathrm{H}), 7.23(\mathrm{~s}, 1 \mathrm{H}), 7.21(\mathrm{~d}, J=8.0 \mathrm{~Hz}, 2 \mathrm{H}), 7.08-7.04(\mathrm{~m}, 1 \mathrm{H}), 6.73-6.70(\mathrm{~m}, 1 \mathrm{H}), 3.78(\mathrm{~s}, 3 \mathrm{H}), 2.40$ (s, 3H); ${ }^{13} \mathrm{C}$ NMR $\left(101 \mathrm{MHz}, \mathrm{CDCl}_{3}\right): \delta_{\mathrm{C}} 165.4,136.8,132.8,131.8,129.7,128.4,127.4,125.5,122.4$, 120.3, 113.7, 112.6, 101.2, 50.5, 21.2; IR (KBr): v 3096, 2942, 2855, 1694, 1633, 1570, 1507, 1489, 1439, 1397, 1312, 1300,1263, 1218, 1186, 1109, 1035, 1019, 822, 758, 728, $673 \mathrm{~cm}^{-1}$; HRMS (ESI-TOF, $m / z$ ): Calcd for $\mathrm{C}_{17} \mathrm{H}_{15} \mathrm{NNaO}_{2}[\mathrm{M}+\mathrm{Na}]^{+}:$288.0995, found 288.1004.

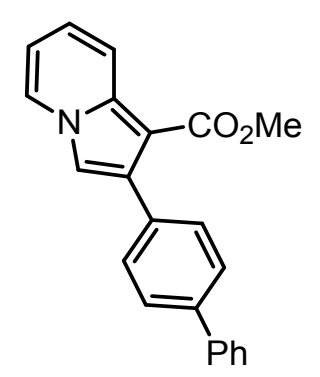

Methyl 2-([1,1'-biphenyl]-4-yl)indolizine-1-carboxylate (3m): Purification of the crude product by column chromatography (silica gel; petroleum ether/ethyl acetate $15: 1$ ) afforded $3 \mathrm{~m}$ as a white solid (131 mg, 80\% yield); $\mathrm{mp} 181-182^{\circ} \mathrm{C}$; ${ }^{1} \mathrm{H}$ NMR $\left(400 \mathrm{MHz}, \mathrm{CDCl}_{3}\right): \delta_{\mathrm{H}} 8.24(\mathrm{~d}, J=9.2 \mathrm{~Hz}, 1 \mathrm{H}), 8.00(\mathrm{~d}, J=6.8 \mathrm{~Hz}, 1 \mathrm{H})$, 7.67-7.59 (m, 6H), 7.48-7.44 (m, 2H), 7.37-7.33 (m, 1H), 7.30 (s, 1H), 7.10-7.06 $(\mathrm{m}, 1 \mathrm{H}), 6.73-6.76(\mathrm{~m}, 1 \mathrm{H}), 3.81(\mathrm{~s}, 3 \mathrm{H}) ;{ }^{13} \mathrm{C} \mathrm{NMR}\left(101 \mathrm{MHz}, \mathrm{CDCl}_{3}\right): \delta_{\mathrm{C}} 165.4,141.1,139.9,136.9$, $133.9,132.4,130.2,128.8,127.2,127.1,126.4,125.5,122.5,120.4,113.9,112.7,101.3,50.6$; IR (KBr): v3126, 2998, 2946, 1702, 1632, 1504, 1481, 1439, 1411, 1368, 1302, 1277, 1223, 1129, 1111, 1038, 1015, 751, 690, $632 \mathrm{~cm}^{-1}$; HRMS (ESI-TOF, $\mathrm{m} / \mathrm{z}$ ): Calcd for $\mathrm{C}_{22} \mathrm{H}_{17} \mathrm{NNaO}_{2}[\mathrm{M}+\mathrm{Na}]^{+}: 350.1151$, found 350.1156 .

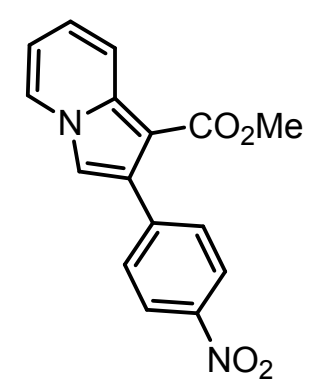

Methyl 2-(4-nitrophenyl)indolizine-1-carboxylate (3n): Purification of the crude product by column chromatography (silica gel; petroleum ether/ethyl acetate 10:1) afforded $3 \mathrm{n}$ as a white solid (127 mg, 86\% yield); $\mathrm{mp} 183-184{ }^{\circ} \mathrm{C} ;{ }^{1} \mathrm{H}$ NMR $\left(400 \mathrm{MHz}, \mathrm{CDCl}_{3}\right): \delta_{\mathrm{H}} 8.25(\mathrm{~d}, J=8.4 \mathrm{~Hz}, 2 \mathrm{H}), 8.24(\mathrm{~d}, J=7.2 \mathrm{~Hz}, 1 \mathrm{H}), 8.02(\mathrm{~d}, J=$ $6.8 \mathrm{~Hz}, 1 \mathrm{H}), 7.67(\mathrm{~d}, J=8.8 \mathrm{~Hz}, 2 \mathrm{H}), 7.32(\mathrm{~s}, 1 \mathrm{H}), 7.15-7.11(\mathrm{~m}, 1 \mathrm{H}), 6.81-6.78$ (m, 1H), $3.80(\mathrm{~s}, 3 \mathrm{H}) ;{ }^{13} \mathrm{C}$ NMR $\left(101 \mathrm{MHz}, \mathrm{CDCl}_{3}\right): \delta_{\mathrm{C}} 165.0,147.0,142.0,137.1,130.6,130.4,125.7$, 123.3, 122.9, 120.6, 114.1, 113.3, 101.3, 50.8; IR (KBr): v3114, 2998, 2959, 1680, 1599, 1508, 1477, $1451,1442,1398,1376,1304,1268,1237,1140,1106,1048,860,786,733,708 \mathrm{~cm}^{-1}$; HRMS (ESI-TOF, $m / z$ ): Calcd for $\mathrm{C}_{16} \mathrm{H}_{12} \mathrm{~N}_{2} \mathrm{NaO}_{4}[\mathrm{M}+\mathrm{Na}]^{+}:$319.0689, found 319.0695. 


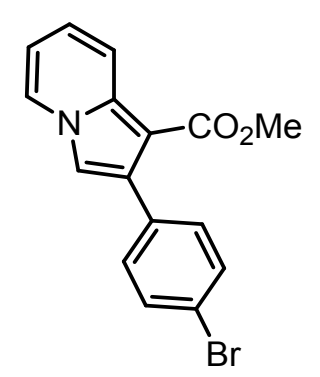

Methyl 2-(4-bromophenyl)indolizine-1-carboxylate (30): Purification of the crude product by column chromatography (silica gel; petroleum ether/ethyl acetate $15: 1$ ) afforded 30 as a white solid (145 mg, $88 \%$ yield); $\mathrm{mp} 129-130{ }^{\circ} \mathrm{C}$; ${ }^{1} \mathrm{H} \mathrm{NMR}\left(400 \mathrm{MHz}, \mathrm{CDCl}_{3}\right): \delta_{\mathrm{H}} 8.22(\mathrm{~d}, J=9.2 \mathrm{~Hz}, 1 \mathrm{H}), 7.98(\mathrm{~d}, J=6.8 \mathrm{~Hz}, 1 \mathrm{H}), 7.52$ (d, $J=8.4 \mathrm{~Hz}, 2 \mathrm{H}), 7.38(\mathrm{~d}, J=8.0 \mathrm{~Hz}, 2 \mathrm{H}), 7.24(\mathrm{~s}, 1 \mathrm{H}), 7.10-7.06(\mathrm{~m}, 1 \mathrm{H}), 6.76-$ $6.73(\mathrm{~m}, 1 \mathrm{H}), 3.78(\mathrm{~s}, 3 \mathrm{H}) ;{ }^{13} \mathrm{C}$ NMR $\left(101 \mathrm{MHz}, \mathrm{CDCl}_{3}\right): \delta_{\mathrm{C}} 165.2,136.9,133.8,131.54,131.5,130.8$, 125.5, 122.8, 121.4, 120.4, 113.7, 112.9, 101.2, 50.6; IR (KBr): v3125, 2998, 2948, 2922, 2850, 1670, 1593, 1503, 1479, 1438, 1414, 1309, 1281, 1216, 1130, 1113, 1068, 1032, 1007, 781, 741, $704 \mathrm{~cm}^{-1}$; HRMS (ESI-TOF, $m / z)$ : Calcd for $\mathrm{C}_{16} \mathrm{H}_{12} \mathrm{BrNNaO}_{2}[\mathrm{M}+\mathrm{Na}]^{+}:$351.9944, found 351.9946.

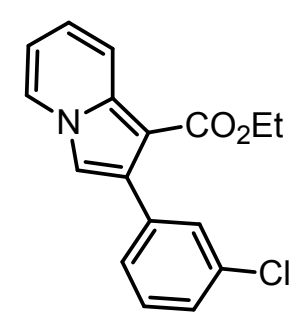

Ethyl 2-(3-chlorophenyl)indolizine-1-carboxylate (3p): Purification of the crude product by column chromatography (silica gel; petroleum ether/ethyl acetate 15:1) afforded $3 p$ as a white solid (129 $\mathrm{mg}, 86 \%$ yield); $\mathrm{mp} 116-117^{\circ} \mathrm{C} ;{ }^{1} \mathrm{H}$ NMR $\left(400 \mathrm{MHz}, \mathrm{CDCl}_{3}\right): 8.26(\mathrm{~d}, J=9.2 \mathrm{~Hz}, 1 \mathrm{H}), 7.98(\mathrm{~d}, J=6.8 \mathrm{~Hz}, 1 \mathrm{H}), 7.51(\mathrm{~s}, 1 \mathrm{H})$, 7.41-7.37 (m, 1H), 7.33-7.28 (m, 2H), 7.24 (s, 1H), $7.08(\mathrm{dd}, J=9.2,6.8 \mathrm{~Hz}, 1 \mathrm{H})$, 6.76-6.73 (m, 1H), $4.26(\mathrm{q}, J=7.2 \mathrm{~Hz}, 2 \mathrm{H}), 1.24(\mathrm{t}, J=7.2 \mathrm{~Hz}, 3 \mathrm{H}) ;{ }^{13} \mathrm{C}$ NMR $\left(101 \mathrm{MHz}, \mathrm{CDCl}_{3}\right): \delta_{\mathrm{C}}$ $164.8,136.9,136.8,133.2,131.1,130.0,128.7,128.2,127.0,125.5,120.4,113.7,112.9,101.5,59.4$, 14.2; IR (KBr): v 3128, 2985, 2917, 1668, 1599, 1501, 1446, 1438, 1401, 1383, 1302, 1278, 1262, 1219, 1114, 1077, 1041, 780, 745, 693, $670 \mathrm{~cm}^{-1}$; HRMS (ESI-TOF, $\mathrm{m} / \mathrm{z}$ ): Calcd for $\mathrm{C}_{17} \mathrm{H}_{14} \mathrm{ClNNaO}_{2}[\mathrm{M}+$ $\mathrm{Na}]^{+}:$322.0605, found 322.0610 .

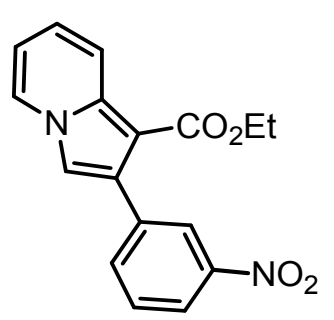

Ethyl 2-(3-nitrophenyl)indolizine-1-carboxylate (3q): Purification of the crude product by column chromatography (silica gel; petroleum ether/ethyl acetate 10:1) afforded $3 q$ as a white solid (137 $\mathrm{mg}, 88 \%$ yield); $\mathrm{mp} 131-132{ }^{\circ} \mathrm{C} ;{ }^{1} \mathrm{H}$ $\operatorname{NMR}\left(400 \mathrm{MHz}, \mathrm{CDCl}_{3}\right): \delta_{\mathrm{H}} 8.39(\mathrm{~s}, 1 \mathrm{H}), 8.27(\mathrm{~d}, J=9.2 \mathrm{~Hz}, 1 \mathrm{H}), 8.21-8.19(\mathrm{~m}$, $1 \mathrm{H}), 8.02(\mathrm{~d}, J=6.8 \mathrm{~Hz}, 1 \mathrm{H}), 7.87(\mathrm{~d}, J=7.6 \mathrm{~Hz}, 1 \mathrm{H}), 7.57-7.53(\mathrm{~m}, 1 \mathrm{H}), 7.32(\mathrm{~s}$, $1 \mathrm{H}), 7.14-7.10(\mathrm{~m}, 1 \mathrm{H}), 6.80-6.77(\mathrm{~m}, 1 \mathrm{H}), 4.26(\mathrm{q}, J=7.2 \mathrm{~Hz}, 2 \mathrm{H}), 1.23(\mathrm{t}, J=7.2 \mathrm{~Hz}, 3 \mathrm{H}) ;{ }^{13} \mathrm{C} \mathrm{NMR}$ $\left(101 \mathrm{MHz}, \mathrm{CDCl}_{3}\right): \delta_{\mathrm{C}} 164.6,147.7,137.0,136.8,136.2,130.1,128.3,125.6,124.8,123.1,121.9$, $120.5,113.9,113.2,101.5,59.6,14.2$; IR (KBr): v 3121, 3070, 2980, 1678, 1631, 1577, 1528, 1501, 
1421, 1378, 1348, 1301, 1269, 1237, 1145, 1046, 786, 745, $687 \mathrm{~cm}^{-1}$; HRMS (ESI-TOF, $\mathrm{m} / \mathrm{z}$ ): Calcd for $\mathrm{C}_{17} \mathrm{H}_{14} \mathrm{~N}_{2} \mathrm{NaO}_{2}[\mathrm{M}+\mathrm{Na}]^{+}:$333.0846, found 333.0844.

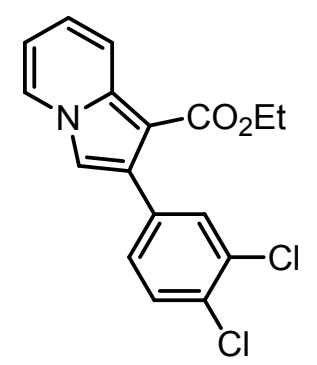

Ethyl 2-(3,4-dichlorophenyl)indolizine-1-carboxylate (3r): Purification of the crude product by column chromatography (silica gel; petroleum ether/ethyl acetate 15:1) afforded $3 \mathrm{r}$ as a white solid (142 $\mathrm{mg}, 85 \%$ yield); $\mathrm{mp} 105-106{ }^{\circ} \mathrm{C}$; ${ }^{1} \mathrm{H} \mathrm{NMR}\left(400 \mathrm{MHz}, \mathrm{CDCl}_{3}\right): \delta_{\mathrm{H}} 8.25(\mathrm{~d}, J=9.2 \mathrm{~Hz}, 1 \mathrm{H}), 7.99(\mathrm{~d}, J=6.8 \mathrm{~Hz}, 1 \mathrm{H}), 7.61$ $(\mathrm{d}, J=2.0 \mathrm{~Hz}, 1 \mathrm{H}), 7.45(\mathrm{~d}, J=8.0 \mathrm{~Hz}, 1 \mathrm{H}), 7.36(\mathrm{dd}, J=8.4,2.0 \mathrm{~Hz}, 1 \mathrm{H}), 7.24(\mathrm{~s}$, $1 \mathrm{H}), 7.10(\mathrm{dd}, J=8.8,6.8 \mathrm{~Hz}, 1 \mathrm{H}), 6.78-6.74(\mathrm{~m}, 1 \mathrm{H}), 4.27(\mathrm{q}, J=7.2 \mathrm{~Hz}, 2 \mathrm{H}), 1.27(\mathrm{t}, J=7.2 \mathrm{~Hz}, 3 \mathrm{H})$; ${ }^{13} \mathrm{C}$ NMR $\left(101 \mathrm{MHz}, \mathrm{CDCl}_{3}\right): \delta_{\mathrm{C}} 165.7,136.9,135.1,131.7,131.5,131.1,130.1,129.41,129.4,125.5$, $122.9,120.4,113.7,113.0,101.4,59.5,14.3$; IR (KBr): $v 3127,3049,2983,2913,1678,1594,1546$, 1500, 1464, 1429, 1388, 1300, 1220, 1134, 1112, 936, 891, 836, 783, 756, $658 \mathrm{~cm}^{-1}$; HRMS (ESI-TOF, $m / z$ ): Calcd for $\mathrm{C}_{17} \mathrm{H}_{13} \mathrm{Cl}_{2} \mathrm{NNaO}_{2}[\mathrm{M}+\mathrm{Na}]^{+}:$356.0216, found 356.0223.

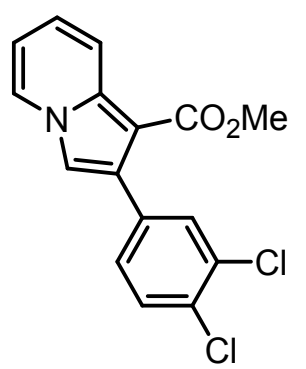

Methyl 2-(3,4-dichlorophenyl)indolizine-1-carboxylate (3s): Purification of the crude product by column chromatography (silica gel; petroleum ether/ethyl acetate $15: 1$ ) afforded $3 \mathrm{~s}$ as a white solid (134 $\mathrm{mg}, 84 \%$ yield); $\mathrm{mp} 144-145^{\circ} \mathrm{C}$; ${ }^{1} \mathrm{H} \mathrm{NMR}\left(400 \mathrm{MHz}, \mathrm{CDCl}_{3}\right): \delta_{\mathrm{H}} 8.23(\mathrm{~d}, J=8.8 \mathrm{~Hz}, 1 \mathrm{H}), 7.99(\mathrm{~d}, J=6.8 \mathrm{~Hz}, 1 \mathrm{H}), 7.60$ (s, 1H), $7.45(\mathrm{~d}, J=8.4 \mathrm{~Hz}, 1 \mathrm{H}), 7.36(\mathrm{~d}, J=8.0 \mathrm{~Hz}, 1 \mathrm{H}), 7.25(\mathrm{~s}, 1 \mathrm{H}), 7.12-7.08(\mathrm{~m}$, 1H), 6.78-6.75 (m, 1H), $3.80(\mathrm{~s}, 3 \mathrm{H}) ;{ }^{13} \mathrm{C}$ NMR (101 MHz, $\left.\mathrm{CDCl}_{3}\right): \delta_{\mathrm{C}} 165.0,136.9,135.0,131.6,131.5$, $131.2,130.2,129.5,129.4,125.6,123.0,120.5,113.9,113.1,101.2,50.7 ; \mathrm{IR}(\mathrm{KBr}): v 3077,2989$, $2948,1689,1600,1503,1467,1442,1417,1370,1305,1223,1132,1115,1045,1032,927,876,782$, 740, $688 \mathrm{~cm}^{-1}$; HRMS (ESI-TOF, $\mathrm{m} / \mathrm{z}$ ): Calcd for $\mathrm{C}_{16} \mathrm{H}_{11} \mathrm{Cl}_{2} \mathrm{NNaO}_{2}[\mathrm{M}+\mathrm{Na}]^{+}: 342.0059$, found 342.0063 .

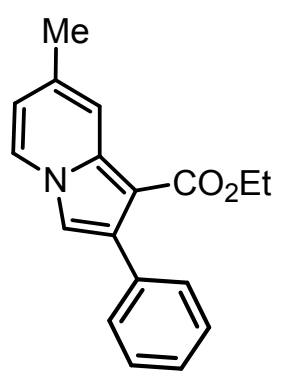

Ethyl 7-methyl-2-phenylindolizine-1-carboxylate (3t): Purification of the crude product by column chromatography (silica gel; petroleum ether/ethyl acetate 15:1) afforded $3 \mathrm{t}$ as a white solid (109 $\mathrm{mg}, 78 \%$ yield); $\mathrm{mp} 108-109^{\circ} \mathrm{C} ;{ }^{1} \mathrm{H}$ NMR $\left(400 \mathrm{MHz}, \mathrm{CDCl}_{3}\right): \delta_{\mathrm{H}} 8.04(\mathrm{~s}, J=9.2 \mathrm{~Hz}, 1 \mathrm{H}), 7.87(\mathrm{~d}, J=6.8 \mathrm{~Hz}, 1 \mathrm{H}), 7.50(\mathrm{~d}, J=$ $8.0 \mathrm{~Hz}, 2 \mathrm{H}), 7.39-7.30(\mathrm{~m}, 3 \mathrm{H}), 7.15(\mathrm{~s}, 1 \mathrm{H}), 6.57(\mathrm{~d}, J=7.2 \mathrm{~Hz}, 1 \mathrm{H}), 4.23$ (q, J = $7.2 \mathrm{~Hz}, 2 \mathrm{H}), 2.39(\mathrm{~s}, 3 \mathrm{H}), 1.20(\mathrm{t}, J=7.2 \mathrm{~Hz}, 3 \mathrm{H}) ;{ }^{13} \mathrm{C} \mathrm{NMR}\left(101 \mathrm{MHz}, \mathrm{CDCl}_{3}\right): \delta_{\mathrm{C}} 165.2,137.4,135.2$, 
$133.4,132.5,129.9,127.5,126.9,125.0,118.7,115.3,113.0,100.2,59.2,21.5,14.3 ; \mathrm{IR}(\mathrm{KBr}): v 3060$, 2962, 2923, 2852, 1677, 1643, 1604, 1556, 1509, 1446, 1383, 1321, 1261, 1238, 1204, 1173, 1137, 1109, 1041, 795, 698, $663 \mathrm{~cm}^{-1}$; HRMS (ESI-TOF, $\mathrm{m} / \mathrm{z}$ ): Calcd for $\mathrm{C}_{18} \mathrm{H}_{17} \mathrm{NNaO}_{2}[\mathrm{M}+\mathrm{Na}]^{+}: 302.1151$, found 302.1148 .

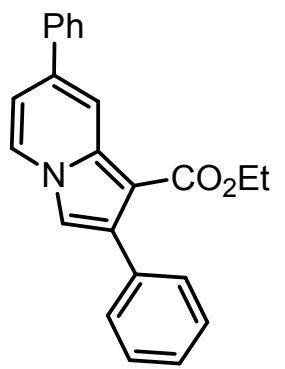

Ethyl 2,7-diphenylindolizine-1-carboxylate (3u): Purification of the crude product by column chromatography (silica gel; petroleum ether/ethyl acetate 15:1) afforded $3 \mathrm{u}$ as a white solid (131 $\mathrm{mg}, 77 \%$ yield); mp $112-113^{\circ} \mathrm{C} ;{ }^{1} \mathrm{H}$ NMR (400 $\left.\mathrm{MHz}, \mathrm{CDCl}_{3}\right): \delta_{\mathrm{H}} 8.54(\mathrm{~s}, 1 \mathrm{H}), 8.04(\mathrm{~d}, J=7.2 \mathrm{~Hz}, 1 \mathrm{H}), 7.71(\mathrm{~d}, J=8.0 \mathrm{~Hz}, 2 \mathrm{H}), 7.53$ $(\mathrm{d}, J=8.0 \mathrm{~Hz}, 2 \mathrm{H}), 7.50-7.46(\mathrm{~m}, 2 \mathrm{H}), 7.41-7.33(\mathrm{~m}, 2 \mathrm{H}), 7.26(\mathrm{~s}, 1 \mathrm{H}), 7.05(\mathrm{~d}, J=$ $6.4 \mathrm{~Hz}, 1 \mathrm{H}), 4.27(\mathrm{q}, J=7.2 \mathrm{~Hz}, 2 \mathrm{H}), 1.23(\mathrm{t}, J=7.2 \mathrm{~Hz}, 3 \mathrm{H}) ;{ }^{13} \mathrm{C} \mathrm{NMR}\left(101 \mathrm{MHz}, \mathrm{CDCl}_{3}\right): \delta_{\mathrm{C}}$ 165.0, 139.0, $137.2,135.2,135.0,133.3,129.9,129.0,128.0,127.5,127.1,126.7,125.6,117.3,113.4,112.4,102.2$, 59.3, 14.3; IR (KBr): v3059, 2959, 2922, 2850, 1676, 1637, 1603, 1509, 1465, 1411, 1384, 1374, 1299, 1249, 1219, 1143, 1112, 1046, 759, $697 \mathrm{~cm}^{-1}$; HRMS (ESI-TOF, $\mathrm{m} / \mathrm{z}$ ): Calcd for $\mathrm{C}_{23} \mathrm{H}_{19} \mathrm{NNaO}_{2}[\mathrm{M}+\mathrm{Na}]^{+}$: 364.1308, found 364.1316.

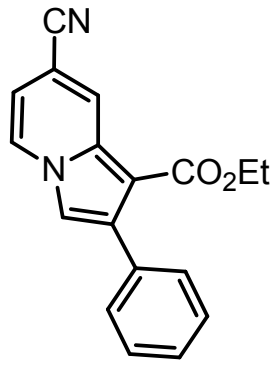

Ethyl 7-cyano-2-phenylindolizine-1-carboxylate (3v): Purification of the crude product by column chromatography (silica gel; petroleum ether/ethyl acetate $15: 1)$ afforded $3 v$ as a white solid (118 $\mathrm{mg}, 81 \%$ yield); mp $108-109{ }^{\circ} \mathrm{C} ;{ }^{1} \mathrm{H}$ NMR $\left(400 \mathrm{MHz}, \mathrm{CDCl}_{3}\right): \delta_{\mathrm{H}} 8.66(\mathrm{~s}, 1 \mathrm{H}, \mathrm{ArH}), 8.03(\mathrm{~d}, J=7.2 \mathrm{~Hz}, 1 \mathrm{H}), 7.49(\mathrm{~d}, J=7.6 \mathrm{~Hz}$, 2H), 7.42-7.36 (m, 4H), $6.83(\mathrm{~d}, J=7.2 \mathrm{~Hz}, 1 \mathrm{H}), 4.29(\mathrm{q}, J=7.2 \mathrm{~Hz}, 2 \mathrm{H}), 1.25(\mathrm{t}, J=$ $7.2 \mathrm{~Hz}, 3 \mathrm{H}) ;{ }^{13} \mathrm{C}$ NMR $\left(101 \mathrm{MHz}^{\mathrm{CDCl}} 3\right.$ ): $\delta_{\mathrm{C}} 163.9,134.9,133.7,133.6,129.83,127.8,127.7,127.2$, $125.8,118.3,116.4,112.3,106.0,104.4,60.1,14.2 ; \mathrm{IR}(\mathrm{KBr}): v 3116,3087,3059,2981,2923,2223$, $1686,1628,1507,1491,1472,1413,1371,1326,1292,1275,1223,1208,1119,1037,1025,803$, 755, $700 \mathrm{~cm}^{-1}$; HRMS (ESI-TOF, $\mathrm{m} / z$ ): Calcd for $\mathrm{C}_{18} \mathrm{H}_{14} \mathrm{~N}_{2} \mathrm{NaO}_{2}[\mathrm{M}+\mathrm{Na}]^{+}:$313.0947, found 313.0952.

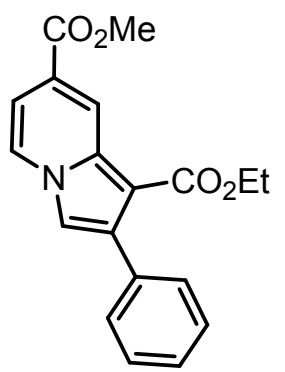

1-Ethyl 7-methyl 2-phenylindolizine-1,7-dicarboxylate (3w): Purification of the crude product by column chromatography (silica gel; petroleum ether/ethyl acetate $15: 1$ ) afforded $3 w$ as a white solid (134 $\mathrm{mg}, 83 \%$ yield); $\mathrm{mp} 110-111^{\circ} \mathrm{C}$; 
${ }^{1} \mathrm{H} \mathrm{NMR}\left(400 \mathrm{MHz}, \mathrm{CDCl}_{3}\right): \delta_{\mathrm{H}} 8.99(\mathrm{~s}, 1 \mathrm{H}), 8.00(\mathrm{~d}, J=7.2 \mathrm{~Hz}, 1 \mathrm{H}), 7.51(\mathrm{~d}, J=7.6 \mathrm{~Hz}, 2 \mathrm{H}), 7.42-7.34$ $(\mathrm{m}, 4 \mathrm{H}), 7.31(\mathrm{~d}, J=7.2 \mathrm{~Hz}, 1 \mathrm{H}), 4.29(\mathrm{q}, J=7.2 \mathrm{~Hz}, 2 \mathrm{H}), 3.96(\mathrm{~s}, 3 \mathrm{H}), 1.26(\mathrm{t}, J=7.2 \mathrm{~Hz}, 3 \mathrm{H}) ;{ }^{13} \mathrm{C}$ NMR $\left(101 \mathrm{MHz}, \mathrm{CDCl}_{3}\right): \delta_{\mathrm{C}} 166.0,164.4,134.8,134.3,134.26,129.9,127.5,127.4,124.9,123.6,123.3$, 115.5, 111.8, 105.7, 59.8, 52.4, 14.2; IR (KBr): v 3119, 3066, 2983, 2923, 2850, 1718, 1680, 1630, 1507, 1467, 1414, 1373, 1335, 1277, 1231, 1143, 1111, 1045, 759, $694 \mathrm{~cm}^{-1}$; HRMS (ESI-TOF, $\mathrm{m} / \mathrm{z}$ ): Calcd for $\mathrm{C}_{19} \mathrm{H}_{17} \mathrm{NNaO}_{4}[\mathrm{M}+\mathrm{Na}]^{+}:$346.1050, found 346.1055.

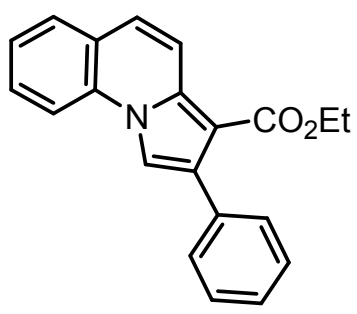

Ethyl 2-phenylpyrrolo[1,2-a]quinoline-3-carboxylate (3x): Purification of the crude product by column chromatography (silica gel; petroleum ether/ethyl acetate 15:1) afforded $\mathbf{3 x}$ as a white solid (143 $\mathrm{mg}$, 91\% yield); mp $117-118{ }^{\circ} \mathrm{C} ;{ }^{1} \mathrm{H}$ NMR $\left(400 \mathrm{MHz}, \mathrm{CDCl}_{3}\right): \delta_{\mathrm{H}} 8.21(\mathrm{~d}, J=9.6 \mathrm{~Hz}, 1 \mathrm{H}), 7.91$ (d, $J=8.4 \mathrm{~Hz}, 1 \mathrm{H}), 7.78(\mathrm{~s}, 1 \mathrm{H}), 7.74(\mathrm{~d}, J=8.0 \mathrm{~Hz}, 1 \mathrm{H}), 7.60-7.54(\mathrm{~m}, 3 \mathrm{H})$, 7.43-7.33 (m, 5H), $4.27(\mathrm{q}, J=7.2 \mathrm{~Hz}, 2 \mathrm{H}), 1.23(\mathrm{t}, J=7.2 \mathrm{~Hz}, 3 \mathrm{H}) ;{ }^{13} \mathrm{C} \mathrm{NMR}\left(101 \mathrm{MHz}, \mathrm{CDCl}_{3}\right): \delta_{\mathrm{C}}$ $165.1,135.2,134.9,132.3,131.3,129.9,128.9,128.8,127.6,127.0,124.6,124.0,123.6,118.9,114.5$, 112.3, 105.2, 59.5, 14.2; IR (KBr): v 3130, 3049, 2979, 2932, 2851, 1676, 1610, 1601, 1544, 1481, 1448, 1420, 1342, 1300, 1272, 1201, 1134, 1056, 805, 752, $700,658 \mathrm{~cm}^{-1}$; HRMS (ESI-TOF, m/z): Calcd for $\mathrm{C}_{21} \mathrm{H}_{17} \mathrm{NNaO}_{2}[\mathrm{M}+\mathrm{Na}]^{+}:$338.1151, found 338.1153.

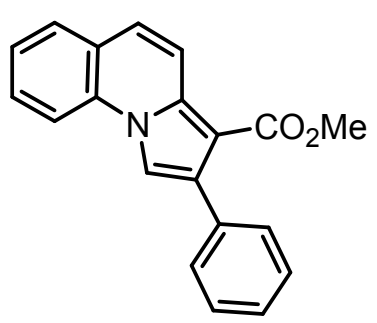

Methyl 2-phenylpyrrolo[1,2-a]quinoline-3-carboxylate (3y): Purification of the crude product by column chromatography (silica gel; petroleum ether/ethyl acetate 15:1) afforded $\mathbf{3 y}$ as a white solid (131 $\mathrm{mg}, 87 \%$ yield); mp $153-154{ }^{\circ} \mathrm{C} ;{ }^{1} \mathrm{H}$ NMR $\left(400 \mathrm{MHz}, \mathrm{CDCl}_{3}\right): \delta_{\mathrm{H}} 8.20(\mathrm{~d}, J=9.2 \mathrm{~Hz}, 1 \mathrm{H}), 7.93$ (d, $J=8.4 \mathrm{~Hz}, 1 \mathrm{H}), 7.79(\mathrm{~s}, 1 \mathrm{H}), 7.75(\mathrm{~d}, J=8.0 \mathrm{~Hz}, 1 \mathrm{H}), 7.61-7.54(\mathrm{~m}, 3 \mathrm{H})$, 7.44-7.34 (m, 5H), $3.80(\mathrm{~s}, 3 \mathrm{H}) ;{ }^{13} \mathrm{C}$ NMR $\left(101 \mathrm{MHz}, \mathrm{CDCl}_{3}\right): \delta_{\mathrm{C}} 165.5,135.04,135.0,132.3,131.3$, $129.8,128.9,128.8,127.7,127.1,124.7,124.0,123.7,118.9,114.6,112.5,104.9,50.8 ; \mathrm{IR}(\mathrm{KBr}): v$ $3051,3006,2936,1681,1610,1544,1480,1448,1412,1343,1277,1235,1217,1136,1097,1053$, 807, 742, $698 \mathrm{~cm}^{-1}$; HRMS (ESI-TOF, $\mathrm{m} / \mathrm{z}$ ): Calcd for $\mathrm{C}_{20} \mathrm{H}_{15} \mathrm{NNaO}_{2}[\mathrm{M}+\mathrm{Na}]^{+}: 324.0995$, found 324.0998. 


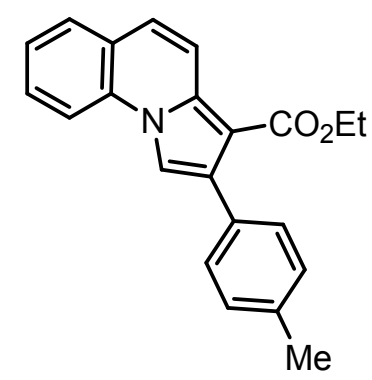

Ethyl 2-(p-tolyl)pyrrolo[1,2-a]quinoline-3-carboxylate (3z): Purification of the crude product by column chromatography (silica gel; petroleum ether/ethyl acetate 15:1) afforded $\mathbf{3 z}$ as a white solid (135 $\mathrm{mg}, 82 \%$ yield); $\mathrm{mp} 136-137^{\circ} \mathrm{C} ;{ }^{1} \mathrm{H}$ NMR $\left(400 \mathrm{MHz}, \mathrm{CDCl}_{3}\right): \delta_{\mathrm{H}} 8.21(\mathrm{~d}, J=9.6 \mathrm{~Hz}, 1 \mathrm{H}), 7.93$ (d, $J=8.4 \mathrm{~Hz}, 1 \mathrm{H}), 7.79(\mathrm{~s}, 1 \mathrm{H}), 7.76(\mathrm{~d}, J=8.0 \mathrm{~Hz}, 1 \mathrm{H}), 7.62-7.58(\mathrm{~m}, 1 \mathrm{H})$, 7.46-7.41 (m, 3H), $7.38(\mathrm{~d}, J=9.6 \mathrm{~Hz}, 1 \mathrm{H}), 7.23(\mathrm{~d}, J=8.0 \mathrm{~Hz}, 2 \mathrm{H}), 4.29(\mathrm{q}, J=7.2 \mathrm{~Hz}, 2 \mathrm{H}), 2.42(\mathrm{~s}, 3 \mathrm{H})$, $1.27(\mathrm{t}, J=7.2 \mathrm{~Hz}, 3 \mathrm{H}) ;{ }^{13} \mathrm{C} \mathrm{NMR}\left(101 \mathrm{MHz}, \mathrm{CDCl}_{3}\right): \delta_{\mathrm{C}} 165.1,136.7,134.8,132.4,132.1,131.4,129.7$, $128.9,128.7,128.4,124.6,124.0,123.5,119.0,114.6,112.3,105.3,59.6,21.2,14.3 ; \mathrm{IR}(\mathrm{KBr}): v 3133$, $3048,2976,2923,1675,1611,1543,1496,1448,1424,1404,1343,1274,1232,1202,1135,1097$, 1053, 805, 743, $682 \mathrm{~cm}^{-1}$; HRMS (ESI-TOF, $\mathrm{m} / \mathrm{z}$ ): Calcd for $\mathrm{C}_{22} \mathrm{H}_{19} \mathrm{NNaO}_{2}[\mathrm{M}+\mathrm{Na}]^{+}: 352.1308$, found 352.1312 .

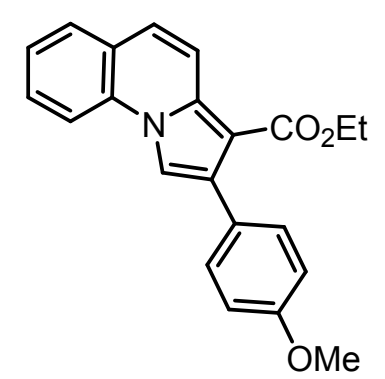

Ethyl 2-(4-methoxyphenyl)pyrrolo[1,2-a]quinoline-3-carboxylate (3aa): Purification of the crude product by column chromatography (silica gel; petroleum ether/ethyl acetate 12:1) afforded 3aa as a white solid (140 mg, $81 \%$ yield); mp $175-176{ }^{\circ} \mathrm{C} ;{ }^{1} \mathrm{H} N M R\left(400 \mathrm{MHz}, \mathrm{CDCl}_{3}\right): \delta_{\mathrm{H}} 8.20(\mathrm{~d}, J=9.6 \mathrm{~Hz}$, 1H), $7.92(\mathrm{~d}, J=8.4 \mathrm{~Hz}, 1 \mathrm{H}), 7.75(\mathrm{~d}, J=9.6 \mathrm{~Hz}, 2 \mathrm{H}), 7.60-7.56(\mathrm{~m}, 1 \mathrm{H}), 7.49$ $(\mathrm{d}, J=8.8 \mathrm{~Hz}, 2 \mathrm{H}), 7.43-7.40(\mathrm{~m}, 1 \mathrm{H}), 7.36(\mathrm{~d}, J=9.2 \mathrm{~Hz}, 1 \mathrm{H}), 6.96(\mathrm{~d}, J=8.4 \mathrm{~Hz}, 2 \mathrm{H}), 4.29(\mathrm{q}, J=7.2$ $\mathrm{Hz}, 2 \mathrm{H}), 3.86(\mathrm{~s}, 3 \mathrm{H}), 1.25(\mathrm{t}, J=7.2 \mathrm{~Hz}, 3 \mathrm{H}) ;{ }^{13} \mathrm{C} \mathrm{NMR}\left(101 \mathrm{MHz}, \mathrm{CDCl}_{3}\right): \delta_{\mathrm{C}} 165.1,158.9,134.8,132.3$, $131.1,131.0,128.9,128.7,127.5,124.5,124.0,123.5,119.0,114.5,113.1,112.2,105.2,59.5,55.3$, 14.3; IR (KBr): v 3122, 2977, 2963, 2931, 1668, 1611, 1559, 1534, 1494, 1449, 1409, 1343, 1274, 1243, 1180, 1149, 1099, 1055, 815, 755, $685 \mathrm{~cm}^{-1}$; HRMS (ESI-TOF, $\mathrm{m} / \mathrm{z}$ ): Calcd for $\mathrm{C}_{22} \mathrm{H}_{19} \mathrm{NNaO}_{2}[\mathrm{M}+$ $\mathrm{Na}]^{+}:$368.1257, found 368.1253 .

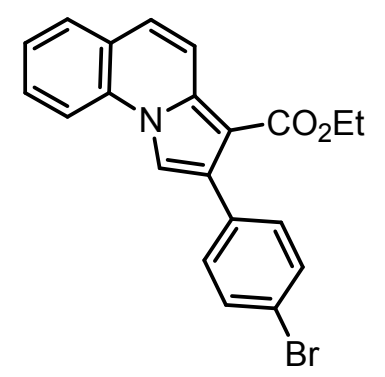

Ethyl 2-(4-bromophenyl)pyrrolo[1,2-a]quinoline-3-carboxylate (3ab): Purification of the crude product by column chromatography (silica gel; petroleum ether/ethyl acetate 15:1) afforded 3ab as a white solid (172 mg, $87 \%$ yield); mp $151-152{ }^{\circ} \mathrm{C} ;{ }^{1} \mathrm{H} N M R\left(400 \mathrm{MHz}, \mathrm{CDCl}_{3}\right): \delta_{\mathrm{H}} 8.21(\mathrm{~d}, J=9.6 \mathrm{~Hz}$, $1 \mathrm{H}), 7.93(\mathrm{~d}, J=8.4 \mathrm{~Hz}, 1 \mathrm{H}), 7.76(\mathrm{~d}, J=9.2 \mathrm{~Hz}, 2 \mathrm{H}), 7.62-7.59(\mathrm{~m}, 1 \mathrm{H}), 7.53$ $(\mathrm{d}, J=8.4 \mathrm{~Hz}, 2 \mathrm{H}), 7.46-7.38(\mathrm{~m}, 4 \mathrm{H}), 4.29(\mathrm{q}, J=7.2 \mathrm{~Hz}, 2 \mathrm{H}), 1.26(\mathrm{t}, J=7.2 \mathrm{~Hz}, 3 \mathrm{H}) ;{ }^{13} \mathrm{C}$ NMR $(101$ 
$\left.\mathrm{MHz}, \mathrm{CDCl}_{3}\right): \delta_{\mathrm{C}} 164.9,135.0,134.1,132.3,131.5,130.7,130.1,129.0,128.9,124.8,124.0,123.9$, 121.2, 118.9, 114.6, 112.3, 105.1, 59.7, 14.3; IR (KBr): v 3120, 3066, 2978, 2923, 1701, 1612, 1545, 1481, 1426, 1383, 1340, 1275, 1220, 1201, 1127, 1063, 1012, 796, 740, $688 \mathrm{~cm}^{-1}$; HRMS (ESI-TOF, $m / z)$ : Calcd for $\mathrm{C}_{21} \mathrm{H}_{16} \mathrm{BrNNaO}_{2}[\mathrm{M}+\mathrm{Na}]^{+}:$416.0257, found 416.0263 .

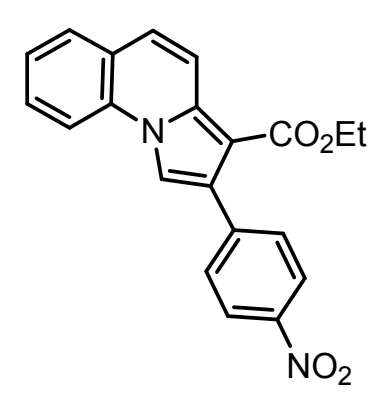

Ethyl 2-(4-nitrophenyl)pyrrolo[1,2-a]quinoline-3-carboxylate (3ac): Purification of the crude product by column chromatography (silica gel; petroleum ether/ethyl acetate 10:1) afforded 3ac as a white solid (155 mg, $86 \%$ yield); mp $189-190{ }^{\circ} \mathrm{C} ;{ }^{1} \mathrm{H}$ NMR $\left(400 \mathrm{MHz}, \mathrm{CDCl}_{3}\right): \delta_{\mathrm{H}} 8.27$ (d, J $=8.4 \mathrm{~Hz}$, 2H), $8.23(\mathrm{~d}, J=9.6 \mathrm{~Hz}, 1 \mathrm{H}), 7.97(\mathrm{~d}, J=8.4 \mathrm{~Hz}, 1 \mathrm{H}), 7.86(\mathrm{~s}, 1 \mathrm{H}), 7.79(\mathrm{~d}, J=$ $8.0 \mathrm{~Hz}, 1 \mathrm{H}), 7.72(\mathrm{~d}, J=8.4 \mathrm{~Hz}, 2 \mathrm{H}), 7.66-7.62(\mathrm{~m}, 1 \mathrm{H}), 7.50-7.46(\mathrm{~m}, 1 \mathrm{H})$, $7.44(\mathrm{~d}, J=9.6 \mathrm{~Hz}, 1 \mathrm{H}), 4.30(\mathrm{q}, J=7.2 \mathrm{~Hz}, 2 \mathrm{H}), 1.27(\mathrm{t}, J=7.2 \mathrm{~Hz}, 3 \mathrm{H}) ;{ }^{13} \mathrm{C} \mathrm{NMR}\left(101 \mathrm{MHz}, \mathrm{CDCl}_{3}\right): \delta_{\mathrm{C}}$ $164.6,146.9,143.5,142.3,135.3,132.2,130.7,129.1,125.1,124.5,124.0,122.9,118.8,114.5,112.8$, 105.0, 59.9, 14.3; IR (KBr): v 3066, 2983, 2923, 2852, 1698, 1600, 1509, 1436, 1402, 1343, 1295, $1272,1202,1128,1105,1047,859,796,741,697 \mathrm{~cm}^{-1}$; HRMS (ESI-TOF, $\mathrm{m} / \mathrm{z}$ ): Calcd for $\mathrm{C}_{21} \mathrm{H}_{16} \mathrm{~N}_{2} \mathrm{NaO}_{4}$ $[\mathrm{M}+\mathrm{Na}]^{+}: 383.1002$, found 383.1011 .

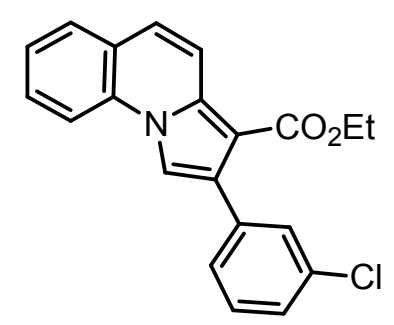

Ethyl 2-(3-chlorophenyl)pyrrolo[1,2-a]quinoline-3-carboxylate (3ad): Purification of the crude product by column chromatography (silica gel; petroleum ether/ethyl acetate 15:1) afforded 3ad as a white solid (147 mg, $84 \%$ yield); mp 100-101 ${ }^{\circ} \mathrm{C} ;{ }^{1} \mathrm{H}$ NMR $\left(400 \mathrm{MHz}, \mathrm{CDCl}_{3}\right): \delta_{\mathrm{H}} 8.23(\mathrm{~d}, J=8.4$ $\mathrm{Hz}, 1 \mathrm{H}), 7.94(\mathrm{~d}, J=8.4 \mathrm{~Hz}, 1 \mathrm{H}), 7.79(\mathrm{~s}, 1 \mathrm{H}), 7.77(\mathrm{~d}, J=8.0 \mathrm{~Hz}, 1 \mathrm{H}), 7.63-$ $7.59(\mathrm{~m}, 1 \mathrm{H}), 7.55(\mathrm{~s}, 1 \mathrm{H}), 7.46-7.43(\mathrm{~m}, 2 \mathrm{H}), 7.40(\mathrm{~d}, J=9.6 \mathrm{~Hz}, 1 \mathrm{H}), 7.33(\mathrm{~d}, J=4.8 \mathrm{~Hz}, 2 \mathrm{H}), 7.28$ (q, J $=7.2 \mathrm{~Hz}, 2 \mathrm{H}), 1.25(\mathrm{t}, J=7.2 \mathrm{~Hz}, 3 \mathrm{H}) ;{ }^{13} \mathrm{C}$ NMR $\left(101 \mathrm{MHz}, \mathrm{CDCl}_{3}\right): \delta_{\mathrm{C}} 164.9,137.0,135.1,133.4,132.3$, $130.0,129.8,129.0,128.9,128.8,128.2,127.0,124.8,124.03,123.98,118.9,114.5,112.5,105.1$, 59.7, 14.2; IR (KBr): v3132, 2957, 2924, 2852, 1672, 1611, 1600, 1542, 1479, 1433, 1384, 1279, 1218, 1201, 1140, 1103, 1054, 808, 742, 712, $692 \mathrm{~cm}^{-1}$; HRMS (ESI-TOF, $\mathrm{m} / \mathrm{z}$ ): Calcd for $\mathrm{C}_{21} \mathrm{H}_{16} \mathrm{ClNNaO}_{2}[\mathrm{M}+$ $\mathrm{Na}]^{+}:$372.0762, found 372.0766 . 


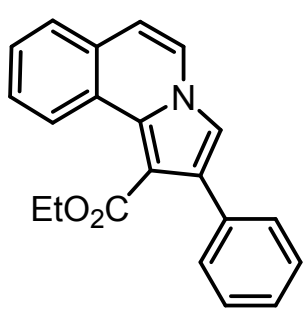

Ethyl 2-phenylpyrrolo[2,1-a]isoquinoline-1-carboxylate (3ae): Purification of the crude product by column chromatography (silica gel; petroleum ether/ethyl acetate 15:1) afforded 3ae as a white solid (140 mg, 89\% yield); mp 94-95 ${ }^{\circ} \mathrm{C} ;{ }^{1} \mathrm{H}$ NMR $\left(400 \mathrm{MHz}, \mathrm{CDCl}_{3}\right): \delta_{\mathrm{H}} 8.94(\mathrm{~d}, J=8.0 \mathrm{~Hz}, 1 \mathrm{H}), 7.75(\mathrm{~d}, J=7.2$ $\mathrm{Hz}, 1 \mathrm{H}), 7.61(\mathrm{~d}, J=7.6 \mathrm{~Hz}, 1 \mathrm{H}), 7.55-7.51(\mathrm{~m}, 1 \mathrm{H}), 7.48-7.37(\mathrm{~m}, 5 \mathrm{H}), 7.34-$ $7.30(\mathrm{~m}, 1 \mathrm{H}), 7.27(\mathrm{~s}, 1 \mathrm{H}), 4.23(\mathrm{q}, J=7.2 \mathrm{~Hz}, 2 \mathrm{H}), 1.05(\mathrm{t}, J=7.2 \mathrm{~Hz}, 3 \mathrm{H}) ;{ }^{13} \mathrm{C} \mathrm{NMR}\left(101 \mathrm{MHz}, \mathrm{CDCl}_{3}\right)$ : $\delta_{\mathrm{C}} 167.5,135.5,130.2,130.0,129.0,128.5,127.9,127.7,127.1,127.05,126.7,125.5,125.0,123.8$, $114.4,113.3,108.1,60.6,13.7$; IR (KBr): v 3062, 2963, 2922, 2850, 1682, 1603, 1505, 1459, 1422, 1372, 1319, 1270, 1212, 1197, 1137, 1105, 1036, 788, 700, $654 \mathrm{~cm}^{-1}$; HRMS (ESI-TOF, $\mathrm{m} / \mathrm{z}$ ): Calcd for $\mathrm{C}_{21} \mathrm{H}_{17} \mathrm{NNaO}_{2}[\mathrm{M}+\mathrm{Na}]^{+}:$338.1151, found 338.1156.

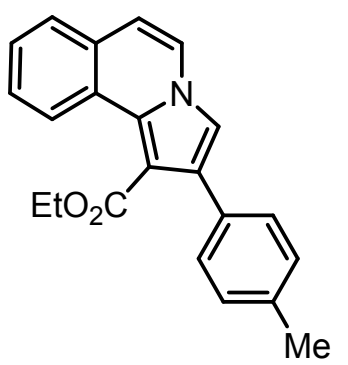

Ethyl 2-(p-tolyl)pyrrolo[2,1-a]isoquinoline-1-carboxylate (3af): Purification of the crude product by column chromatography (silica gel; petroleum ether/ethyl acetate 15:1) afforded 3af as a white solid (142 mg, 86\% yield); mp 100-101 ${ }^{\circ} \mathrm{C} ;{ }^{1} \mathrm{H}$ NMR $\left(400 \mathrm{MHz}, \mathrm{CDCl}_{3}\right): \delta_{\mathrm{H}} 8.88(\mathrm{~d}, J=8.4 \mathrm{~Hz}, 1 \mathrm{H}), 7.72(\mathrm{~d}$, $J=7.2 \mathrm{~Hz}, 1 \mathrm{H}), 7.59(\mathrm{~d}, J=7.6 \mathrm{~Hz}, 1 \mathrm{H}), 7.53-7.49(\mathrm{~m}, 1 \mathrm{H}), 7.46-7.42(\mathrm{~m}, 1 \mathrm{H})$, $7.32(\mathrm{~d}, J=8.0 \mathrm{~Hz}, 2 \mathrm{H}), 7.23(\mathrm{~s}, 1 \mathrm{H}), 7.19(\mathrm{~d}, J=7.6 \mathrm{~Hz}, 2 \mathrm{H}), 6.88(\mathrm{~d}, J=7.2 \mathrm{~Hz}, 1 \mathrm{H}), 4.26(\mathrm{q}, J=7.2 \mathrm{~Hz}$, 2H), $2.40(\mathrm{~s}, 3 \mathrm{~m}), 1.10(\mathrm{t}, J=7.2 \mathrm{~Hz}, 3 \mathrm{H}) ;{ }^{13} \mathrm{C} \mathrm{NMR}\left(101 \mathrm{MHz}, \mathrm{CDCl}_{3}\right): \delta_{\mathrm{C}} 167.5,135.5,130.3,130.0$, 129.0, 128.5, 128.0, 127.7, 127.11, 127.06, 126.7, 125.5, 125.0, 123.8, 114.4, 113.3, 108.1, 60.0, 13.7; IR (KBr): v 3062, 2963, 2922, 2850, 1682, 1603, 1505, 1459, 1422, 1372, 1319, 1270, 1212, 1197, 1137, 1105, 1036, 788, 700, $654 \mathrm{~cm}^{-1}$; HRMS (ESI-TOF, $\mathrm{m} / \mathrm{z}$ ): Calcd for $\mathrm{C}_{22} \mathrm{H}_{19} \mathrm{NNaO}_{2}[\mathrm{M}+\mathrm{Na}]^{+}$: 352.1308 , found 352.1317 .

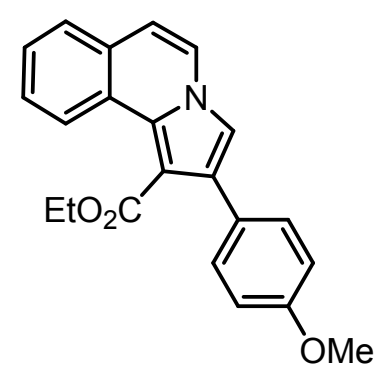

Ethyl 2-(4-methoxyphenyl)pyrrolo[2,1-a]isoquinoline-1-carboxylate (3ag): Purification of the crude product by column chromatography (silica gel; petroleum ether/ethyl acetate 12:1) afforded 3ag as a white solid (152 mg, $88 \%$ yield); mp $139-140{ }^{\circ} \mathrm{C} ;{ }^{1} \mathrm{H} \mathrm{NMR}\left(400 \mathrm{MHz}, \mathrm{CDCl}_{3}\right): \delta_{\mathrm{H}} 8.89(\mathrm{~d}, J=8.4 \mathrm{~Hz}$, $1 \mathrm{H}), 7.73(\mathrm{~d}, J=7.2 \mathrm{~Hz}, 1 \mathrm{H}), 7.60(\mathrm{~d}, J=7.2 \mathrm{~Hz}, 1 \mathrm{H}), 7.53-7.50(\mathrm{~m}, 1 \mathrm{H}), 7.46-$ $7.43(\mathrm{~m}, 1 \mathrm{H}), 7.36(\mathrm{~d}, J=8.4 \mathrm{~Hz}, 2 \mathrm{H}), 7.21(\mathrm{~s}, 1 \mathrm{H}), 6.94(\mathrm{~d}, J=8.4 \mathrm{~Hz}, 2 \mathrm{H}), 6.89(\mathrm{~d}, J=7.2 \mathrm{~Hz}, 1 \mathrm{H}), 4.26$ (q, $J=7.2 \mathrm{~Hz}, 2 \mathrm{H}), 3.85(\mathrm{~s}, 3 \mathrm{~m}), 1.11(\mathrm{t}, J=7.2 \mathrm{~Hz}, 3 \mathrm{H}) ;{ }^{13} \mathrm{C} N M R\left(101 \mathrm{MHz}, \mathrm{CDCl}_{3}\right): \delta_{\mathrm{C}} 167.6,158.7$, 
$130.1,129.6,128.5,127.8,127.6,127.04,127.00,125.5,124.9,123.8,114.2,113.5,113.1,108.0$, 60.6, 55.3, 13.9; IR (KBr): v3063, 2978, 2933, 2840, 1679, 1612, 1558, 1504, 1459, 1441, 1370, 1319, 1272, 1245, 1211, 1196, 1105, 1035, 833, 795, 760, $662 \mathrm{~cm}^{-1}$; HRMS (ESI-TOF, m/z): Calcd for $\mathrm{C}_{22} \mathrm{H}_{19} \mathrm{NNaO}_{3}[\mathrm{M}+\mathrm{Na}]^{+}:$368.1257, found 368.1260.

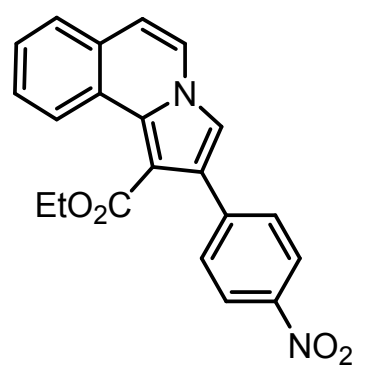

\section{Ethyl 2-(4-nitrophenyl)pyrrolo[2,1-a]isoquinoline-1-carboxylate (3ah):} Purification of the crude product by column chromatography (silica gel; petroleum ether/ethyl acetate 10:1) afforded 3ah as a white solid (157 mg, $87 \%$ yield); mp $136-137{ }^{\circ} \mathrm{C} ;{ }^{1} \mathrm{H}$ NMR $\left(400 \mathrm{MHz}, \mathrm{CDCl}_{3}\right): \delta_{\mathrm{H}} 9.00(\mathrm{~d}, J=8.0 \mathrm{~Hz}$, $1 \mathrm{H}), 8.26(\mathrm{~d}, J=8.8 \mathrm{~Hz}, 2 \mathrm{H}), 7.77(\mathrm{~d}, J=7.2 \mathrm{~Hz}, 1 \mathrm{H}), 7.64(\mathrm{~d}, J=7.6 \mathrm{~Hz}, 1 \mathrm{H})$, 7.59-7.49 (m, 4H), $7.33(\mathrm{~s}, 1 \mathrm{H}), 6.97(\mathrm{~d}, J=7.6 \mathrm{~Hz}, 2 \mathrm{H}), 4.26(\mathrm{q}, J=7.2 \mathrm{~Hz}, 2 \mathrm{H}), 1.10(\mathrm{t}, J=7.2 \mathrm{~Hz}, 3 \mathrm{H})$; ${ }^{13} \mathrm{C}$ NMR $\left(101 \mathrm{MHz}, \mathrm{CDCl}_{3}\right): \delta_{\mathrm{C}} 166.7,146.6,142.7,129.6,128.7,128.0,127.8,127.7,127.2,125.3$, $123.7,123.3,115.1,114.1,60.8,13.8$; IR (KBr): v 3136, 2981, 2901, 1699, 1600, 1509, 1458, 1408, 1373, 1344, 1270, 1211, 1142, 1108, 1034, 796, 752, 704, $662 \mathrm{~cm}^{-1}$; HRMS (ESI-TOF, $\mathrm{m} / \mathrm{z}$ ): Calcd for $\mathrm{C}_{21} \mathrm{H}_{16} \mathrm{~N}_{2} \mathrm{NaO}_{4}[\mathrm{M}+\mathrm{Na}]^{+}:$383.1002, found 383.1014.

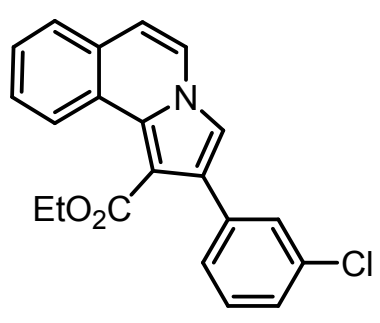

Ethyl 2-(3-chlorophenyl)pyrrolo[2,1-a]isoquinoline-1-carboxylate (3ai): Purification of the crude product by column chromatography (silica gel; petroleum ether/ethyl acetate 15:1) afforded 3ai as a white solid (143 mg, $82 \%$ yield); mp $117-118{ }^{\circ} \mathrm{C} ;{ }^{1} \mathrm{H}$ NMR $\left(400 \mathrm{MHz}, \mathrm{CDCl}_{3}\right): \delta_{\mathrm{H}} 8.23(\mathrm{~d}, J=9.2$ $\mathrm{Hz}, 1 \mathrm{H}), 7.94(\mathrm{~d}, J=8.4 \mathrm{~Hz}, 1 \mathrm{H}), 7.79(\mathrm{~s}, 1 \mathrm{H}), 7.77(\mathrm{~d}, J=8.0 \mathrm{~Hz}, 1 \mathrm{H}), 7.63-$ $7.59(\mathrm{~m}, 1 \mathrm{H}), 7.55(\mathrm{~s}, 1 \mathrm{H}), 7.46-7.43(\mathrm{~m}, 2 \mathrm{H}), 7.40(\mathrm{~d}, J=9.6 \mathrm{~Hz}, 1 \mathrm{H}), 7.33(\mathrm{~d}, J=4.8 \mathrm{~Hz}, 2 \mathrm{H}), 7.28(\mathrm{~d}, J$ $=7.2 \mathrm{~Hz}, 2 \mathrm{H}), 1.25(\mathrm{t}, J=7.2 \mathrm{~Hz}, 3 \mathrm{H}) ;{ }^{13} \mathrm{C} \mathrm{NMR}\left(101 \mathrm{MHz}, \mathrm{CDCl}_{3}\right): \delta_{\mathrm{C}} 167.0,137.4,133.7,130.6,129.2$, $129.1,128.7,128.6,127.8,127.33,127.27,127.1,126.7,125.4,125.2,123.7,114.6,113.6,107.9$, 60.6, 13.7; IR (KBr): v3066, 2979, 2897, 1675, 1599, 1566, 1504, 1478, 1458, 1422, 1370, 1318, 1272, 1218, 1197, 1145, 1108, 1034, 793, 760, 702, $658 \mathrm{~cm}^{-1}$; HRMS (ESI-TOF, $\mathrm{m} / \mathrm{z}$ ): Calcd for $\mathrm{C}_{21} \mathrm{H}_{16} \mathrm{ClNNaO}_{2}[\mathrm{M}+\mathrm{Na}]^{+}: 372.0762$, found 372.0762 . 


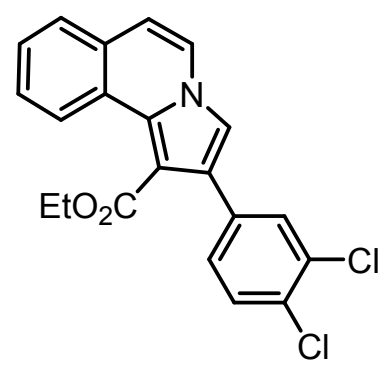

\section{Ethyl-2-(3,4-dichlorophenyl)pyrrolo[2,1-a]isoquinoline-1-carboxylate}

(3aj): Purification of the crude product by column chromatography (silica gel; petroleum ether/ethyl acetate 15:1) afforded 3aj as a white solid (156 $\mathrm{mg}, 81 \%$ yield); mp $139-140{ }^{\circ} \mathrm{C} ;{ }^{1} \mathrm{H}$ NMR $\left(400 \mathrm{MHz}, \mathrm{CDCl}_{3}\right): \delta_{\mathrm{H}} 8.99$ (d, $J=$ $8.4 \mathrm{~Hz}, 1 \mathrm{H}), 7.73(\mathrm{~d}, J=7.2 \mathrm{~Hz}, 1 \mathrm{H}), 7.62(\mathrm{~d}, J=7.6 \mathrm{~Hz}, 1 \mathrm{H}), 7.56-7.44(\mathrm{~m}$, $4 \mathrm{H}), 7.25(\mathrm{~d}, J=7.2 \mathrm{~Hz}, 2 \mathrm{H}), 6.93(\mathrm{~d}, J=7.2 \mathrm{~Hz}, 2 \mathrm{H}), 4.27(\mathrm{q}, J=7.2 \mathrm{~Hz}, 2 \mathrm{H}), 1.14(\mathrm{t}, J=7.2 \mathrm{~Hz}, 3 \mathrm{H}) ;{ }^{13} \mathrm{C}$ NMR $\left(101 \mathrm{MHz}, \mathrm{CDCl}_{3}\right): \delta_{\mathrm{C}} 166.8,135.7,131.9,130.9,130.8,129.8,128.6,128.5,127.8,127.6,127.5$, $127.1,125.34,125.27,123.7,114.7,113.8,107.8,60.7,13.9 ; \mathrm{IR}(\mathrm{KBr}): v 3067,2982,2896,1675$, $1600,1554,1504,1472,1459,1383,1367,1316,1273,1218,1198,1127,1107,1032,795,760,678$ $\mathrm{cm}^{-1}$; HRMS (ESI-TOF, $\mathrm{m} / \mathrm{z}$ ): Calcd for $\mathrm{C}_{21} \mathrm{H}_{15} \mathrm{Cl}_{2} \mathrm{NNaO}_{2}[\mathrm{M}+\mathrm{Na}]^{+}:$: 406.0372, found 406.0376 .

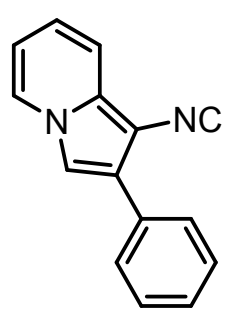

1-Isocyano-2-phenylindolizine (4a): Purification of the crude product by column chromatography (silica gel; petroleum ether/ethyl acetate 12:1) afforded 4 a as a white solid (95 mg, 87\% yield); mp $114-115^{\circ} \mathrm{C} ;{ }^{1} \mathrm{H}$ NMR (400 MHz, $\mathrm{CDCl}_{3}$ ): $\delta_{\mathrm{H}} 7.90$ (d, $J=6.8 \mathrm{~Hz}, 1 \mathrm{H}, \mathrm{ArH}), 7.75-7.73(\mathrm{~m}, 2 \mathrm{H}), 7.54(\mathrm{~d}, J=9.2 \mathrm{~Hz}, 1 \mathrm{H}), 7.49-7.45(\mathrm{~m}, 2 \mathrm{H})$, 7.39-7.35 (m, 1H), $7.33(\mathrm{~s}, 1 \mathrm{H}), 6.95-6.91(\mathrm{~m}, 1 \mathrm{H}), 6.67-6.63(\mathrm{~m}, 1 \mathrm{H}) ;{ }^{13} \mathrm{C}$ NMR $(101$ $\left.\mathrm{MHz}, \mathrm{CDCl}_{3}\right): \delta_{\mathrm{C}} 167.1,131.9,131.4,128.9,127.8,127.6,125.31,125.27,120.3,116.5,112.4,108.6$, 108.0; IR (KBr): v 3129, 3040, 2924, 2106, 1647,, 1602, 1540, 1515, 1478, 1376, 1308, 1257, 1074, 783, 743, 707, $699 \mathrm{~cm}^{-1}$; HRMS (ESI-TOF, $\mathrm{m} / \mathrm{z}$ ): Calcd for $\mathrm{C}_{15} \mathrm{H}_{10} \mathrm{~N}_{2} \mathrm{Na}[\mathrm{M}+\mathrm{Na}]^{+}:$241.0736, found 241.0744.

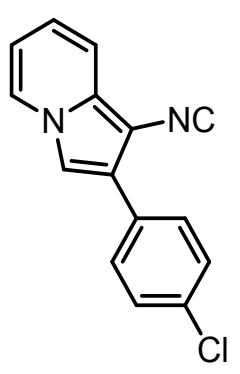

2-(4-Chlorophenyl)-1-isocyanoindolizine (4b): Purification of the crude product by column chromatography (silica gel; petroleum ether/ethyl acetate 12:1) afforded $\mathbf{4 b}$ as a white solid (108 mg, 85\% yield); mp $129-130{ }^{\circ} \mathrm{C} ;{ }^{1} \mathrm{H}$ NMR $\left(400 \mathrm{MHz}, \mathrm{CDCl}_{3}\right): \delta_{\mathrm{H}}$ $7.90(\mathrm{~d}, J=7.2 \mathrm{~Hz}, 1 \mathrm{H}), 7.66(\mathrm{~d}, J=8.4 \mathrm{~Hz}, 2 \mathrm{H}), 7.54(\mathrm{~d}, J=9.2 \mathrm{~Hz}, 1 \mathrm{H}), 7.44(\mathrm{~d}, J=8.4$ $\mathrm{Hz}, 2 \mathrm{H}), 7.31(\mathrm{~s}, 1 \mathrm{H}), 6.94(\mathrm{dd}, J=8.8,6.8 \mathrm{~Hz}, 1 \mathrm{H}), 6.68-6.64(\mathrm{~m}, 1 \mathrm{H}) ;{ }^{13} \mathrm{C}$ NMR $(101$ $\left.\mathrm{MHz}, \mathrm{CDCl}_{3}\right): \delta_{\mathrm{C}} 167.5,133.7,131.4,130.4,129.1,128.8,125.3,124.1,120.6,116.5,112.6,108.6$, 99.9; IR (KBr): v 2960, 2116, 1646, 1627, 1608, 155, 1539, 1520, 1506, 1488, 1456, 1260, 750, 719, $690 \mathrm{~cm}^{-1}$; HRMS (ESI-TOF, $\mathrm{m} / \mathrm{z}$ ): Calcd for $\mathrm{C}_{15} \mathrm{H}_{9} \mathrm{ClN}_{2} \mathrm{Na}$ [M + Na] ${ }^{+}: 275.0346$, found 275.0339 . 


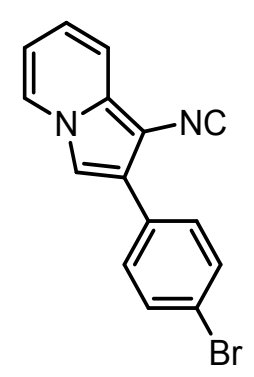

2-(4-Bromophenyl)-1-isocyanoindolizine (4c): Purification of the crude product by column chromatography (silica gel; petroleum ether/ethyl acetate 12:1) afforded 4c as a white solid (125 mg, 84\% yield); mp $141-142{ }^{\circ} \mathrm{C} ;{ }^{1} \mathrm{H}$ NMR (400 MHz, $\mathrm{CDCl}_{3}$ ): $\delta_{\mathrm{H}} 7.90(\mathrm{~d}, J=6.8 \mathrm{~Hz}, 1 \mathrm{H}), 7.60(\mathrm{~s}, 4 \mathrm{H}), 7.54(\mathrm{~d}, J=8.8 \mathrm{~Hz}, 1 \mathrm{H}), 7.31(\mathrm{~s}, 1 \mathrm{H}), 6.95$ $\mathrm{Br}(\mathrm{dd}, J=8.8,6.8 \mathrm{~Hz}, 1 \mathrm{H}), 6.68-6.65(\mathrm{~m}, 1 \mathrm{H}) ;{ }^{13} \mathrm{C} \mathrm{NMR}\left(101 \mathrm{MHz}, \mathrm{CDCl}_{3}\right): \delta_{\mathrm{C}} 167.6$, 132.1, 131.5, 130.8, 129.1, 125.3, 124.1, 121.9, 120.6, 116.5, 112.6, 108.5; IR (KBr): v 2978, 2123 , $1673,1620,1559,1533,1463,1413,1387,1302,1268,1206,1127,1133,1054,1029,1003,776$, 732, $668 \mathrm{~cm}^{-1}$; HRMS (ESI-TOF, $\mathrm{m} / z$ ): Calcd for $\mathrm{C}_{15} \mathrm{H}_{9} \mathrm{BrN}_{2} \mathrm{Na}$ [M + Na] $]^{+}: 318.9841$, found 318.9856 .

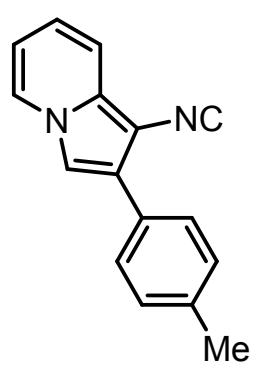

1-Isocyano-2-( $p$-tolyl)indolizine (4d): Purification of the crude product by column chromatography (silica gel; petroleum ether/ethyl acetate 12:1) afforded $\mathbf{4} \mathbf{d}$ as a white solid (100 mg, $86 \%$ yield); $\mathrm{mp} 119-120{ }^{\circ} \mathrm{C} ;{ }^{1} \mathrm{H} \mathrm{NMR}\left(400 \mathrm{MHz}, \mathrm{CDCl}_{3}\right): \delta_{\mathrm{H}} 7.88$ $(\mathrm{d}, J=6.8 \mathrm{~Hz}, 1 \mathrm{H}), 7.63(\mathrm{~d}, J=8.0 \mathrm{~Hz}, 2 \mathrm{H}), 7.52(\mathrm{~d}, J=8.8 \mathrm{~Hz}, 1 \mathrm{H}), 7.29-7.26(\mathrm{~m}$, Me 3H), $6.91(\mathrm{dd}, J=8.8,6.8 \mathrm{~Hz}, 1 \mathrm{H}), 6.64-6.61(\mathrm{~m}, 1 \mathrm{H}), 2.40(\mathrm{~s}, 3 \mathrm{H}) ;{ }^{13} \mathrm{C}$ NMR $(101$ $\left.\mathrm{MHz}, \mathrm{CDCl}_{3}\right): \delta_{\mathrm{C}} 167.0,137.6,131.3,129.6,128.9,127.4,125.4,125.2,120.2,116.4,112.3,10.4,21.3$; IR (KBr): v3127, 2977, 2123, 1656, 1621, 1559, 1517, 1476, 1383, 1315, 1252, 1218, 1064, 1020, 819, 743, 715, $688 \mathrm{~cm}^{-1}$; HRMS (ESI-TOF, $\mathrm{m} / \mathrm{z}$ ): Calcd for $\mathrm{C}_{16} \mathrm{H}_{12} \mathrm{~N}_{2} \mathrm{Na}[\mathrm{M}+\mathrm{Na}]^{+}: 255.0893$, found 255.0887 .

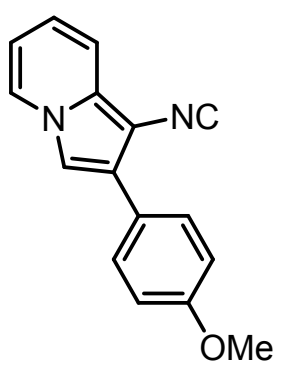

1-Isocyano-2-(4-methoxyphenyl)indolizine (4e): Purification of the crude product by column chromatography (silica gel; petroleum ether/ethyl acetate 10:1) afforded $4 \mathrm{e}$ as a white solid (110 $\mathrm{mg}, 89 \%$ yield); mp $127-128{ }^{\circ} \mathrm{C} ;{ }^{1} \mathrm{H}$ NMR $\left(400 \mathrm{MHz}, \mathrm{CDCl}_{3}\right): \delta_{\mathrm{H}} 7.87(\mathrm{~d}, J=6.8 \mathrm{~Hz}, 1 \mathrm{H}), 7.66(\mathrm{~d}, J=8.4 \mathrm{~Hz}, 2 \mathrm{H}), 7.51(\mathrm{~d}, J=$ $9.2 \mathrm{~Hz}, 1 \mathrm{H}), 7.26(\mathrm{~s}, 1 \mathrm{H}), 7.00(\mathrm{~d}, J=8.8 \mathrm{~Hz}, 2 \mathrm{H}), 6.90(\mathrm{dd}, J=8.8,6.8 \mathrm{~Hz}, 1 \mathrm{H})$, 6.63-6.60 (m, 1H), $3.86(\mathrm{~s}, 3 \mathrm{H}) ;{ }^{13} \mathrm{C}$ NMR $\left(101 \mathrm{MHz}, \mathrm{CDCl}_{3}\right): \delta_{\mathrm{C}} 167.2,159.4,131.3,128.8,125.23$, 125.18, 124.4, 120.1, 116.3, 114.4, 112.2, 108.1, 55.3; IR (KBr): v2998, 2922, 2126, 1672, 1624, 1552, $1501,1479,1438,1386,1315,1279,1203,1155,1108,1028,854,793,766,684 \mathrm{~cm}^{-1}$; HRMS (ESI-TOF, $m / z$ ): Calcd for $\mathrm{C}_{16} \mathrm{H}_{12} \mathrm{~N}_{2} \mathrm{NaO}[\mathrm{M}+\mathrm{Na}]^{+}: 271.0842$, found 271.0851 . 


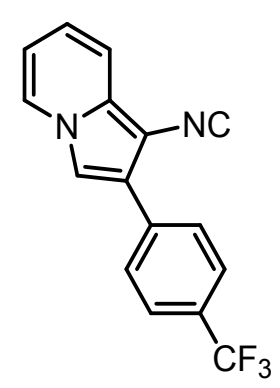

1-Isocyano-2-(4-(trifluoromethyl)phenyl)indolizine (4f): Purification of the crude product by column chromatography (silica gel; petroleum ether/ethyl acetate 12:1) afforded $\mathbf{4 f}$ as a white solid (120 $\mathrm{mg}, 84 \%$ yield); $\mathrm{mp} 152-153^{\circ} \mathrm{C} ;{ }^{1} \mathrm{H}$ NMR $\left(400 \mathrm{MHz}, \mathrm{CDCl}_{3}\right): \delta_{\mathrm{H}} 7.90(\mathrm{~d}, J=6.8 \mathrm{~Hz}, 1 \mathrm{H}), 7.83(\mathrm{~d}, J=8.0 \mathrm{~Hz}, 2 \mathrm{H}), 7.70(\mathrm{~d}, J=$ $8.4 \mathrm{~Hz}, 2 \mathrm{H}), 7.54(\mathrm{~d}, J=8.8 \mathrm{~Hz}, 1 \mathrm{H}), 7.36(\mathrm{~s}, 1 \mathrm{H}), 6.95(\mathrm{dd}, J=8.8,6.8 \mathrm{~Hz}, 1 \mathrm{H})$, 6.69-6.66 (m, 1H); ${ }^{13} \mathrm{C} N M R\left(101 \mathrm{MHz}, \mathrm{CDCl}_{3}\right): \delta_{\mathrm{C}} 167.8,135.5,131.5,129.6\left(q, J_{C-F}=32.4 \mathrm{~Hz}\right), 127.7$, $125.8\left(q, J_{C-F}=3.5 \mathrm{~Hz}\right), 125.5,125.3,123.6\left(q, J_{C-F}=270.5 \mathrm{~Hz}\right), 122.7,120.9,115.6,112.8,109.0$; IR (KBr): $v 3003,2928,2116,1682,1632,1549,1501,1487,1421,1379,1319,1273,1235,1147,1096$, 1057, 1041, 789, 746, $688 \mathrm{~cm}^{-1}$; HRMS (ESI-TOF, $\mathrm{m} / \mathrm{z}$ ): Calcd for $\mathrm{C}_{16} \mathrm{H}_{9} \mathrm{~F}_{3} \mathrm{~N}_{2} \mathrm{Na}[\mathrm{M}+\mathrm{Na}]^{+}: 309.0610$, found 309.0623 .

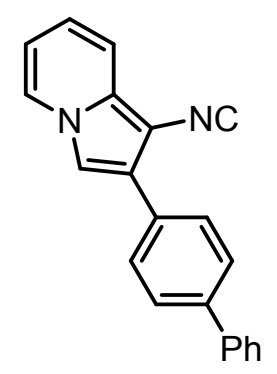

2-([1,1'-Biphenyl]-4-yl)-1-isocyanoindolizine (4g): Purification of the crude product by column chromatography (silica gel; petroleum ether/ethyl acetate 12:1) afforded $4 \mathrm{~g}$ as a white solid (129 $\mathrm{mg}, 88 \%$ yield); $\mathrm{mp} 136-137{ }^{\circ} \mathrm{C} ;{ }^{1} \mathrm{H}$ NMR $\left(400 \mathrm{MHz}, \mathrm{CDCl}_{3}\right): \delta_{\mathrm{H}} 7.90(\mathrm{~d}, J=6.8 \mathrm{~Hz}, 1 \mathrm{H}), 7.82(\mathrm{~d}, J=8.4 \mathrm{~Hz}, 2 \mathrm{H}), 7.70(\mathrm{~d}, J=$ Ph $8.0 \mathrm{~Hz}, 2 \mathrm{H}), 7.65(\mathrm{~d}, J=7.6 \mathrm{~Hz}, 2 \mathrm{H}), 7.55(\mathrm{~d}, J=9.2 \mathrm{~Hz}, 1 \mathrm{H}), 7.49-7.45(\mathrm{~m}, 2 \mathrm{H})$, 7.39-7.35 (m, 2H), $6.93(\mathrm{dd}, J=8.8,6.8 \mathrm{~Hz}, 1 \mathrm{H}), 6.67-6.63(\mathrm{~m}, 1 \mathrm{H}) ;{ }^{13} \mathrm{C}$ NMR $\left(101 \mathrm{MHz}, \mathrm{CDCl}_{3}\right): \delta_{\mathrm{C}}$ $167.4,140.6,13.5,130.9,128.8,127.9,127.6,127.5,127.0,125.3,124.9,120.4,116.5,112.5,108.6$; IR (KBr): v 3105, 3045, 2922, 2113, 1648, 1618, 1479, 1450, 1376, 1259, 1220, 1078, 771, 743, 695 $\mathrm{cm}^{-1}$; HRMS (ESI-TOF, $\mathrm{m} / z$ ): Calcd for $\mathrm{C}_{21} \mathrm{H}_{14} \mathrm{~N}_{2} \mathrm{Na}[\mathrm{M}+\mathrm{Na}]^{+}: 317.1049$, found 317.1058.

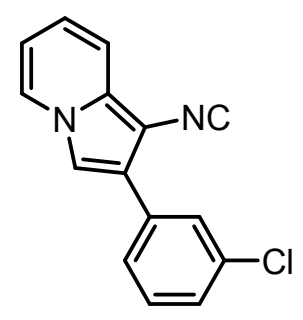

2-(3-Chlorophenyl)-1-isocyanoindolizine (4h): Purification of the crude product by column chromatography (silica gel; petroleum ether/ethyl acetate 12:1) afforded $4 \mathrm{~h}$ as a white solid (115 $\mathrm{mg}, 91 \%$ yield); $\mathrm{mp} 133-134{ }^{\circ} \mathrm{C} ;{ }^{1} \mathrm{H}$ NMR (400 $\left.\mathrm{MHz}, \mathrm{CDCl}_{3}\right): \delta_{\mathrm{H}} 7.89(\mathrm{~d}, J=6.8 \mathrm{~Hz}, 1 \mathrm{H}), 7.67-7.64(\mathrm{~m}, 2 \mathrm{H}), 7.53(\mathrm{~d}, J=8.8 \mathrm{~Hz}, 1 \mathrm{H})$, 7.41-7.37 (m, 1H), $7.34(\mathrm{~d}, J=11.2 \mathrm{~Hz}, 2 \mathrm{H}), 6.94(\mathrm{dd}, J=8.8,6.8 \mathrm{~Hz}, 1 \mathrm{H}), 6.67-$ $6.64(\mathrm{~m}, 1 \mathrm{H}) ;{ }^{13} \mathrm{C}$ NMR $\left(101 \mathrm{MHz}, \mathrm{CDCl}_{3}\right): \delta_{\mathrm{c}} 167.7,134.8,133.7,131.5,130.2,127.8,127.5,125.7$, $125.3,123.9,120.7,116.5,112.7,108.8$; IR (KBr): v 3033, 2944, 2117, 1693, 1597, 1563, 1487, 1452, 1396, 1325, 1268, 1209, 1132, 1109, 1026, 832, 756, 709, $681 \mathrm{~cm}^{-1}$; HRMS (ESI-TOF, m/z): Calcd for $\mathrm{C}_{15} \mathrm{H}_{9} \mathrm{ClN}_{2} \mathrm{Na}[\mathrm{M}+\mathrm{Na}]^{+}: 275.0346$, found 275.0337 . 


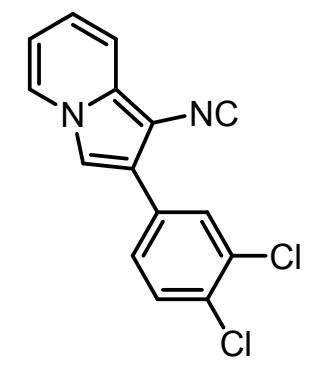

2-(3,4-Dichlorophenyl)-1-isocyanoindolizine (4i): Purification of the crude product by column chromatography (silica gel; petroleum ether/ethyl acetate 12:1) afforded $4 \mathbf{i}$ as a white solid (123 $\mathrm{mg}, 86 \%$ yield); $\mathrm{mp} 139-140{ }^{\circ} \mathrm{C} ;{ }^{1} \mathrm{H}$ NMR $\left(400 \mathrm{MHz}, \mathrm{CDCl}_{3}\right.$ ): $\delta_{\mathrm{H}} 7.90(\mathrm{~d}, J=7.2 \mathrm{~Hz}, 1 \mathrm{H}), 7.78(\mathrm{~s}, 1 \mathrm{H}), 7.60$ (d, J = 8.4 Hz, $1 \mathrm{H}), 7.55-7.52(\mathrm{~m}, 2 \mathrm{H}), 7.32(\mathrm{~s}, 1 \mathrm{H}), 6.96(\mathrm{dd}, J=8.8,6.8 \mathrm{~Hz}, 1 \mathrm{H}), 6.69-6.66(\mathrm{~m}$, 1H); ${ }^{13} \mathrm{C}$ NMR (101 MHz, $\left.\mathrm{CDCl}_{3}\right): \delta_{\mathrm{C}} 167.6,132.0,131.5,130.9,129.9,129.2,126.7,125.3,124.3$, $122.9,120.9,116.6,112.9,108.8 ; \mathrm{IR}(\mathrm{KBr}): v 2959,2133,1648,1611,1577,1560,1508,1474,1420$, 1400, 1384, 1081, 751, 734, $670 \mathrm{~cm}^{-1}$; HRMS (ESI-TOF, $\mathrm{m} / \mathrm{z}$ ): Calcd for $\mathrm{C}_{15} \mathrm{H}_{8} \mathrm{Cl}_{2} \mathrm{~N}_{2} \mathrm{Na}[\mathrm{M}+\mathrm{Na}]^{+}$: 308.9957, found 308.9951 .

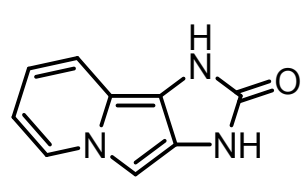

1H-Imidazo[4,5-a]indolizin-2(3H)-one (6a): Purification of the crude product by column chromatography (silica gel; petroleum ether/ethyl acetate 8:1) afforded 6a as a white solid $\left(71 \mathrm{mg}, 79 \%\right.$ yield); mp $102-103{ }^{\circ} \mathrm{C} ;{ }^{1} \mathrm{H}$ NMR $(400 \mathrm{MHz}$, $\left.\mathrm{CDCl}_{3}\right): \delta_{\mathrm{H}} 8.00(\mathrm{~d}, J=6.8 \mathrm{~Hz}, 1 \mathrm{H}), 7.19(\mathrm{~d}, J=8.8 \mathrm{~Hz}, 1 \mathrm{H}), 6.93(\mathrm{t}, J=7.2 \mathrm{~Hz}, 1 \mathrm{H}), 6.62(\mathrm{t}, J=6.8 \mathrm{~Hz}$, 1H), $5.81(\mathrm{~s}, 1 \mathrm{H}), 3.99(\mathrm{~s}, 2 \mathrm{H}) ;{ }^{13} \mathrm{C} N M R\left(101 \mathrm{MHz}, \mathrm{CDCl}_{3}\right): \delta_{\mathrm{C}} 145.8,136.8,125.3,122.9,117.0,114.3$, $110.8,87.9$; IR (KBr): v 3629, 3417, 3242, 2962, 2852, 1630, 1619, 1483, 1400, 1383, 1261, 1096, 1023, 852, 802, 777, 739, 709, 657, $506 \mathrm{~cm}^{-1}$; HRMS (ESI-TOF, $\mathrm{m} / \mathrm{z}$ ): Calcd for $\mathrm{C}_{9} \mathrm{H}_{7} \mathrm{~N}_{3} \mathrm{NaO}[\mathrm{M}+\mathrm{Na}]^{+}$: 196.0481, found 196.0473 .

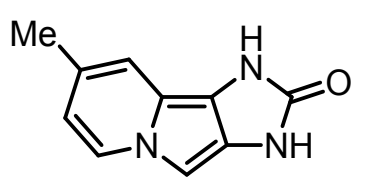

8-Methyl-1H-imidazo[4,5-a]indolizin-2(3H)-one (6b): Purification of the crude product by column chromatography (silica gel; petroleum ether/ethyl acetate $8: 1$ ) afforded $6 \mathbf{b}$ as a white solid (59 $\mathrm{mg}, 63 \%$ yield); $\mathrm{mp} 125-126^{\circ} \mathrm{C}$;

Yield: 63\% (59 mg); white solid, mp $125-126{ }^{\circ} \mathrm{C} ;{ }^{1} \mathrm{H}$ NMR (400 MHz, $\left.\mathrm{CDCl}_{3}\right): \delta_{\mathrm{H}} 7.88(\mathrm{~d}, J=6.8 \mathrm{~Hz}, 1 \mathrm{H})$, $6.96(\mathrm{~s}, 1 \mathrm{H}), 6.45(\mathrm{~d}, J=7.2 \mathrm{~Hz}, 1 \mathrm{H}), 5.68(\mathrm{~s}, 1 \mathrm{H}), 3.96(\mathrm{~s}, 2 \mathrm{H}), 2.30(\mathrm{~s}, 3 \mathrm{H}) ;{ }^{13} \mathrm{C} \mathrm{NMR}\left(101 \mathrm{MHz}, \mathrm{CDCl}_{3}\right)$ : $\delta_{\mathrm{C}} 146.1,137.2,133.8,124.8,115.7,114.7,113.3,86.8 ; \mathrm{IR}(\mathrm{KBr}): v 3550,3423,3347,3243,3131$, $2962,2923,2852,2700,1632,1579,1500,1470,1452,1345,1261,1207,1188,1095,1022,963$, 822, 802, 745, 715, 656, 617, 600, $556 \mathrm{~cm}^{-1}$; HRMS (ESI-TOF, $\mathrm{m} / \mathrm{z}$ ): Calcd for $\mathrm{C}_{10} \mathrm{H}_{9} \mathrm{~N}_{3} \mathrm{NaO}[\mathrm{M}+\mathrm{Na}]^{+}$: 210.0638 , found 210.0629 . 


\section{NMR spectra}

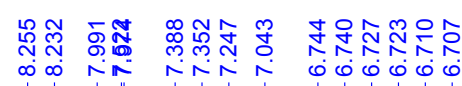

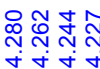

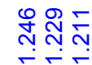

i

$\stackrel{8}{\circ}$

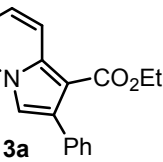

${ }^{1} \mathrm{H} \mathrm{NMR}\left(\mathrm{CDCl}_{3}, 400 \mathrm{MHz}\right)$

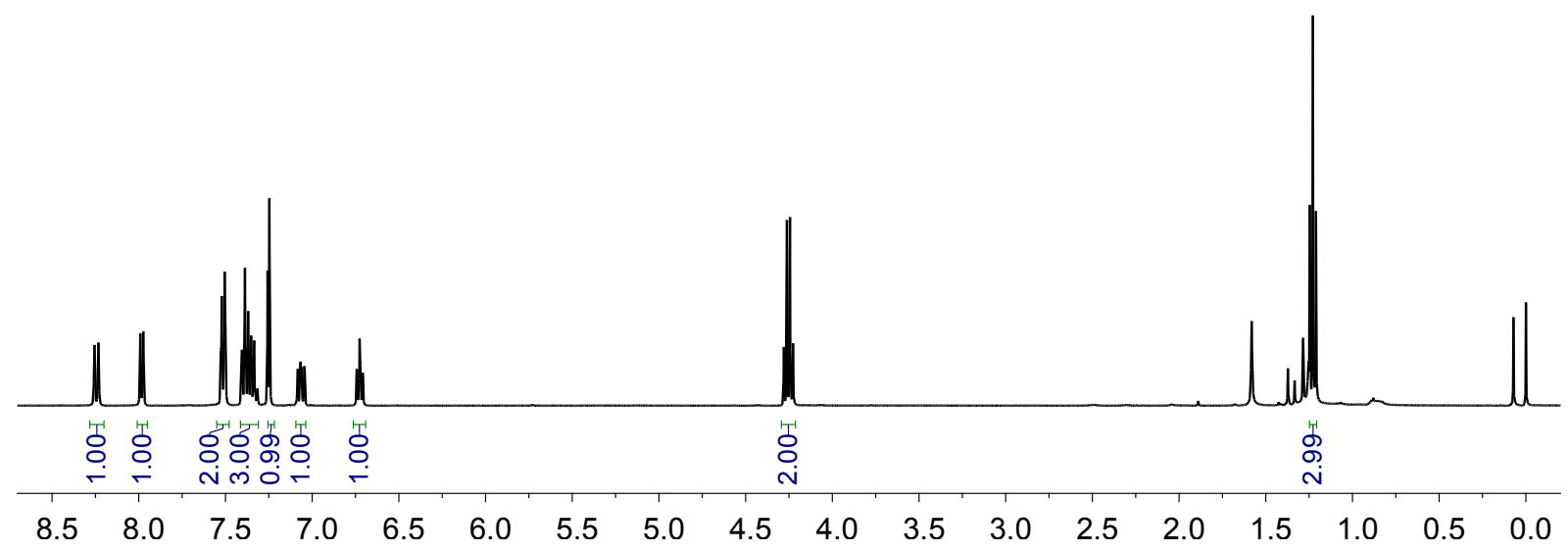

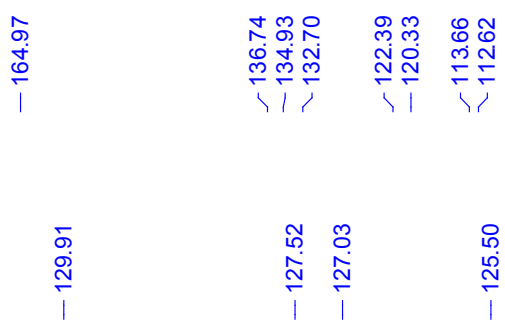

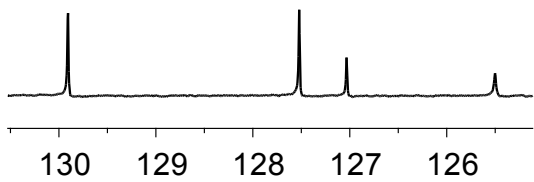

$\frac{1}{\frac{0}{10}}$

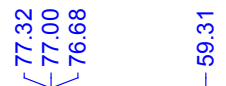

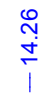

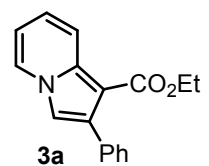

${ }^{13} \mathrm{C}$ NMR $\left(\mathrm{CDCl}_{3}, 101 \mathrm{MHz}\right)$

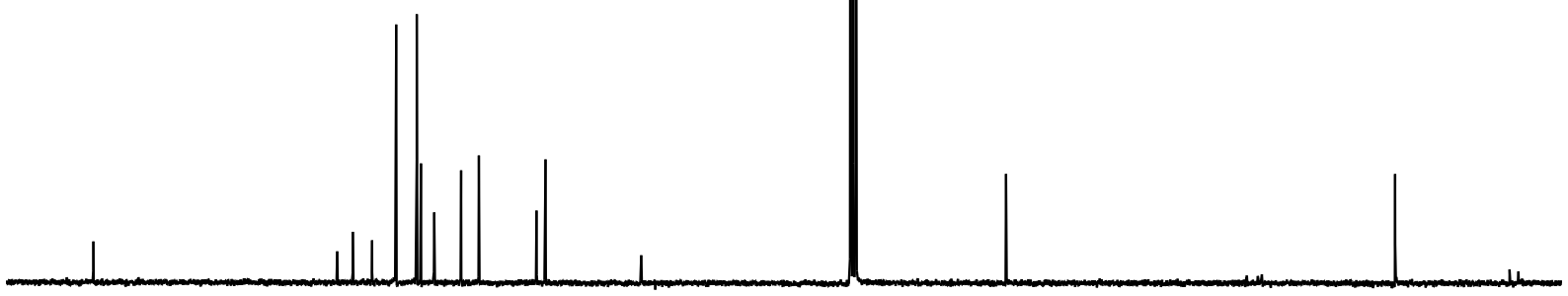

$\begin{array}{llllllllllllllllll}170 & 160 & 150 & 140 & 130 & 120 & 110 & 100 & 90 & 80 & 70 & 60 & 50 & 40 & 30 & 20 & 10 & 0\end{array}$ 


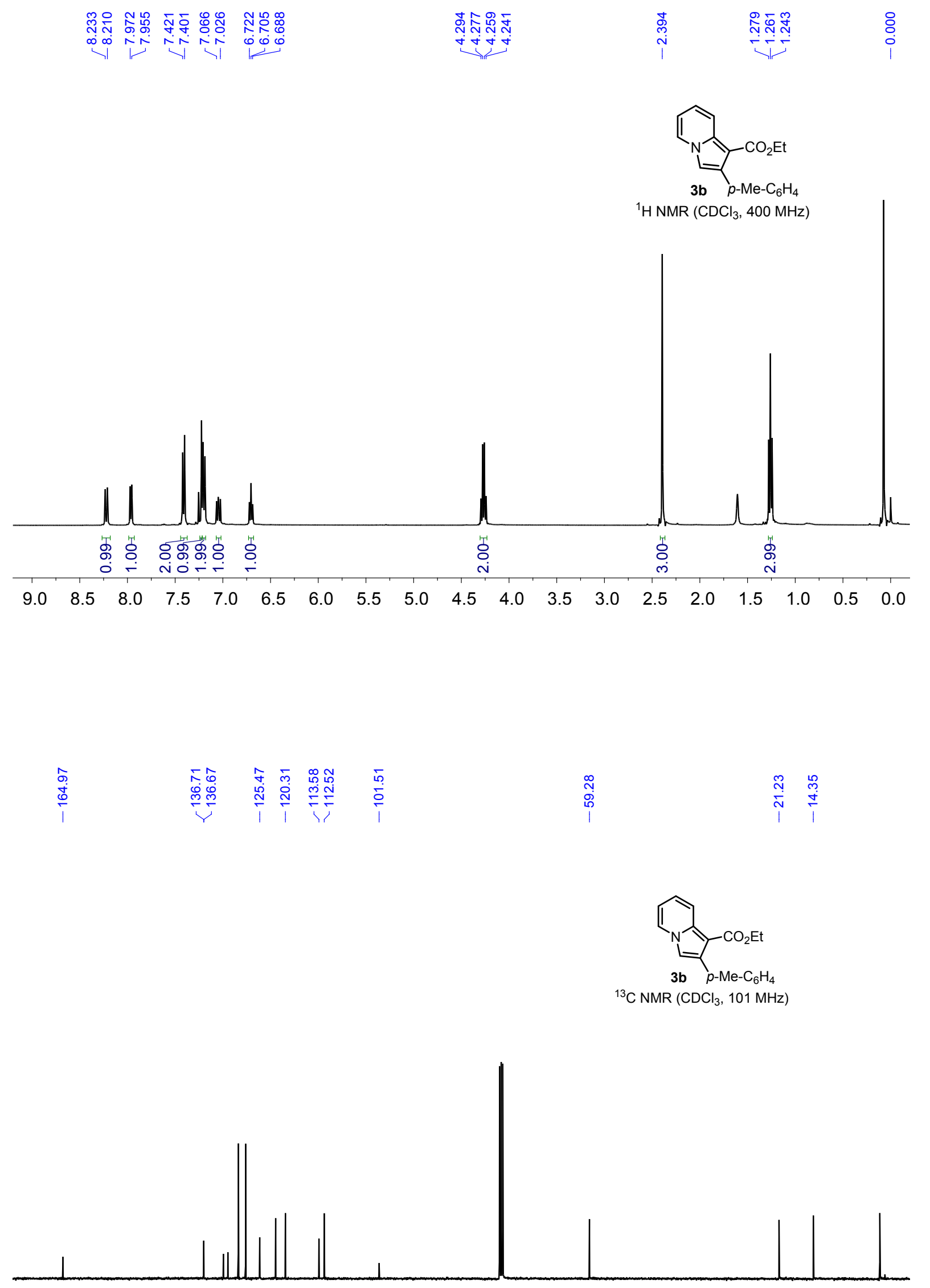

$\begin{array}{llllllllllllllllll}170 & 160 & 150 & 140 & 130 & 120 & 110 & 100 & 90 & 80 & 70 & 60 & 50 & 40 & 30 & 20 & 10 & 0\end{array}$ 


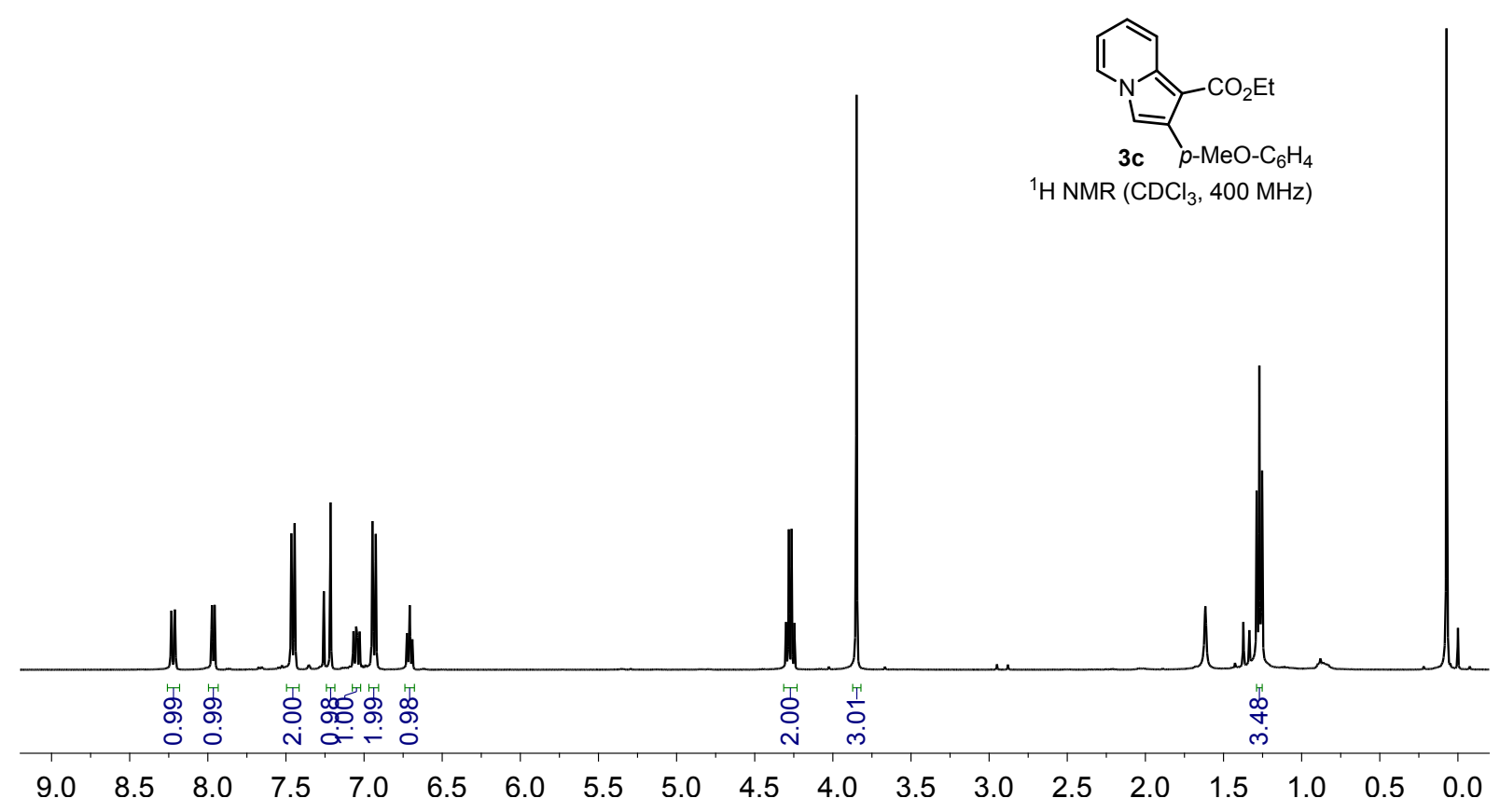

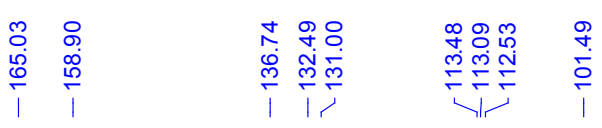

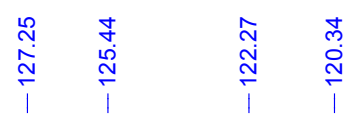

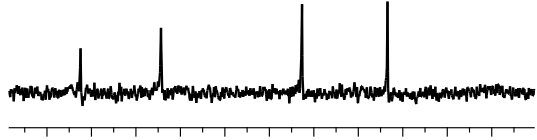

123

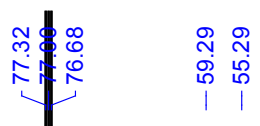

N $\stackrel{\substack{m \\ \dot{\sim}}}{i}$

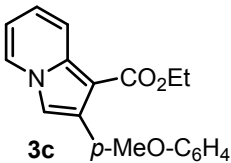

${ }^{13} \mathrm{C} \mathrm{NMR}\left(\mathrm{CDCl}_{3}, 101 \mathrm{MHz}\right)$

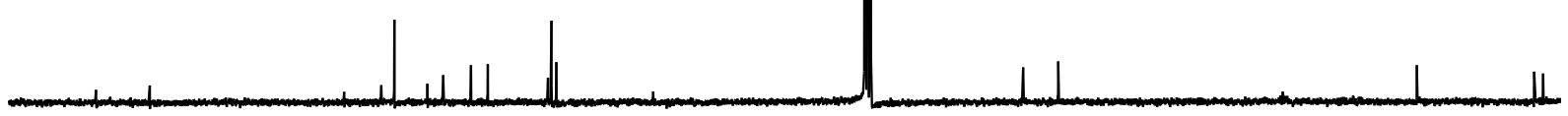

$\begin{array}{lllllllll}170 & 160 & 150 & 140 & 130 & 120 & 110 & 100 & 90\end{array}$

$80 \quad 70 \quad 60$

50 


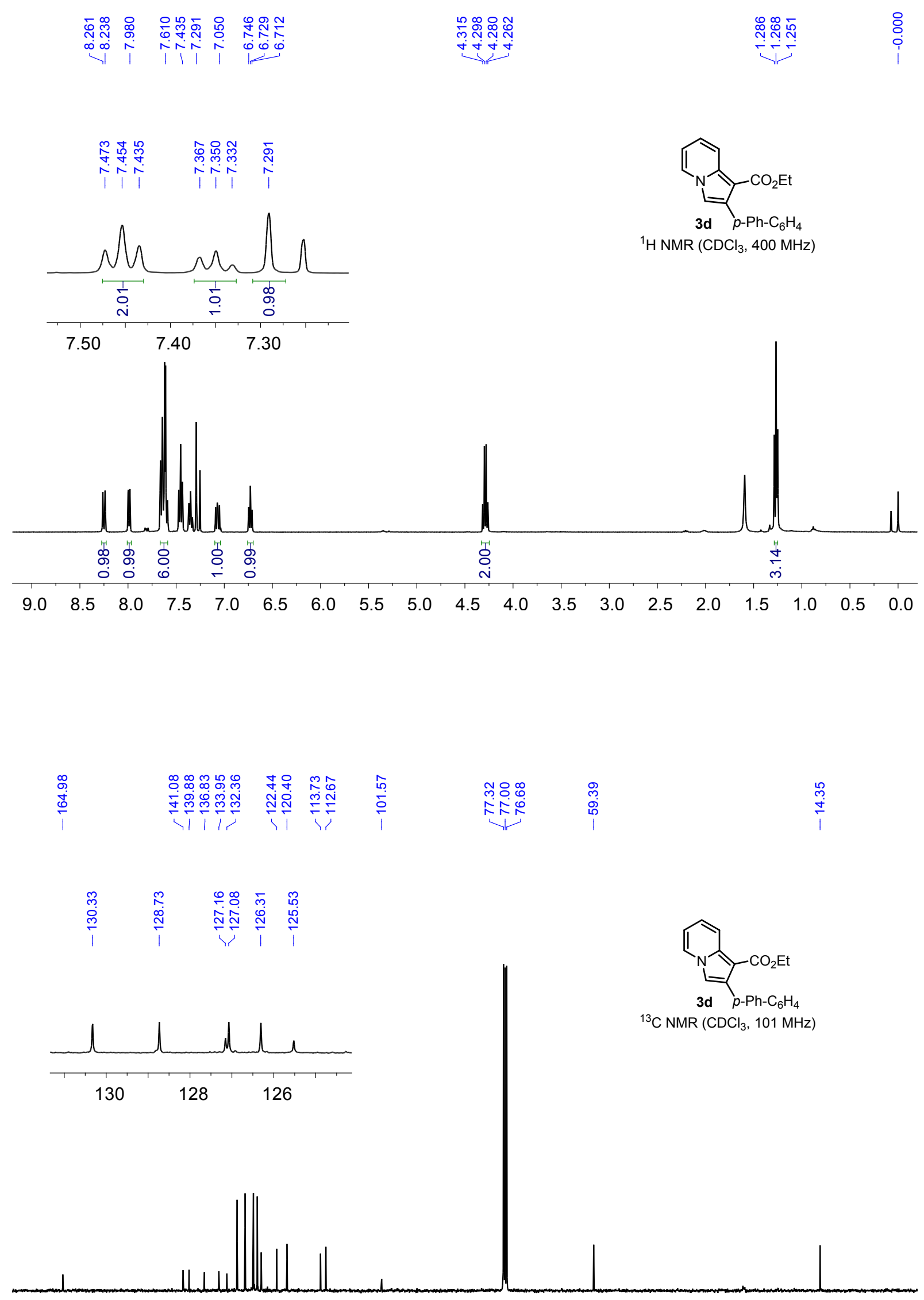

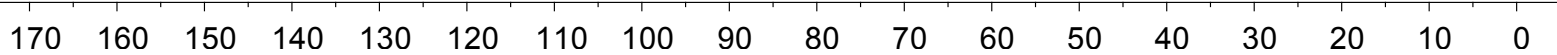




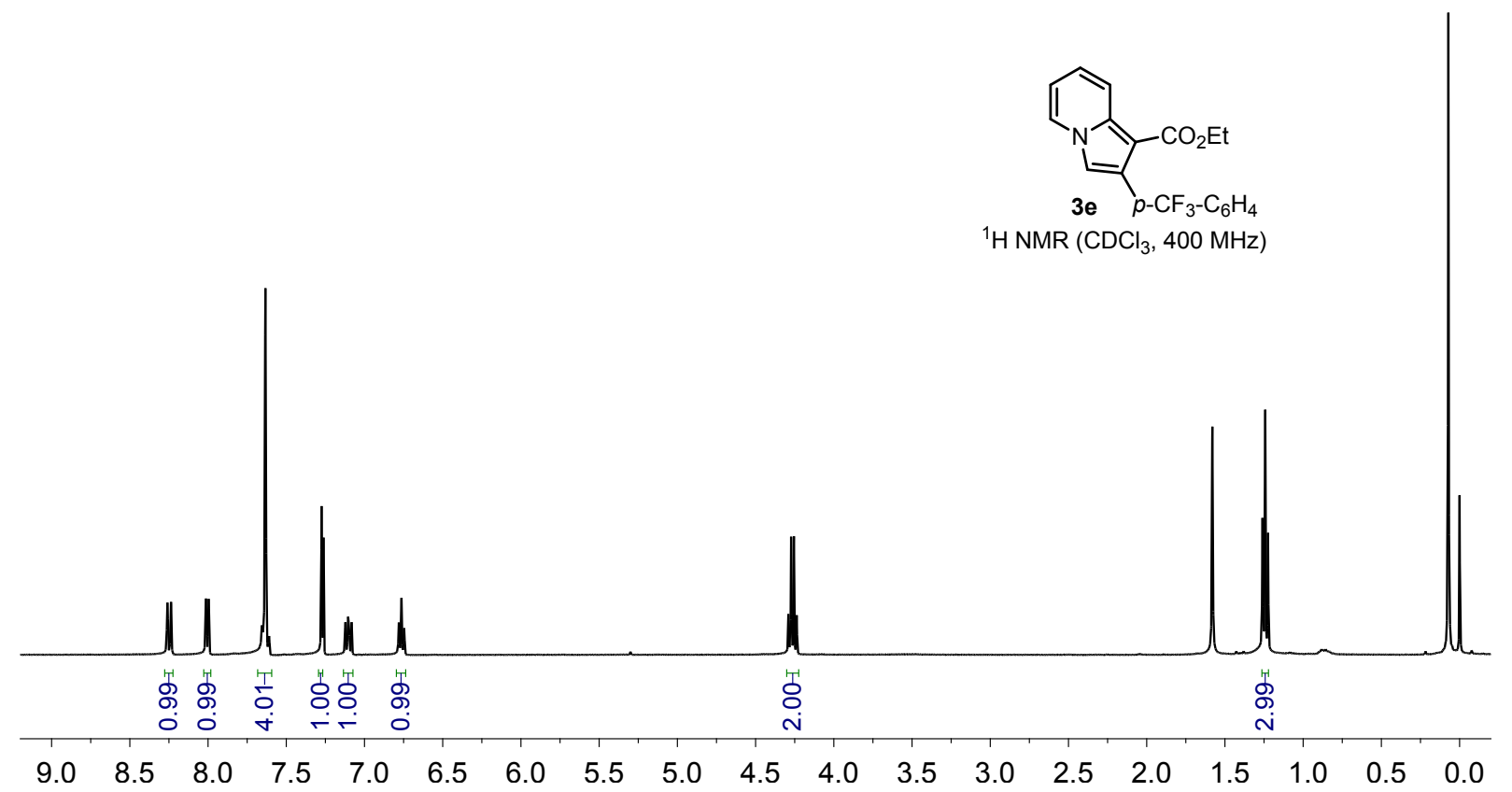

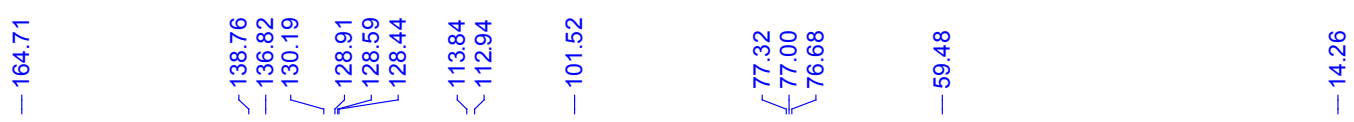

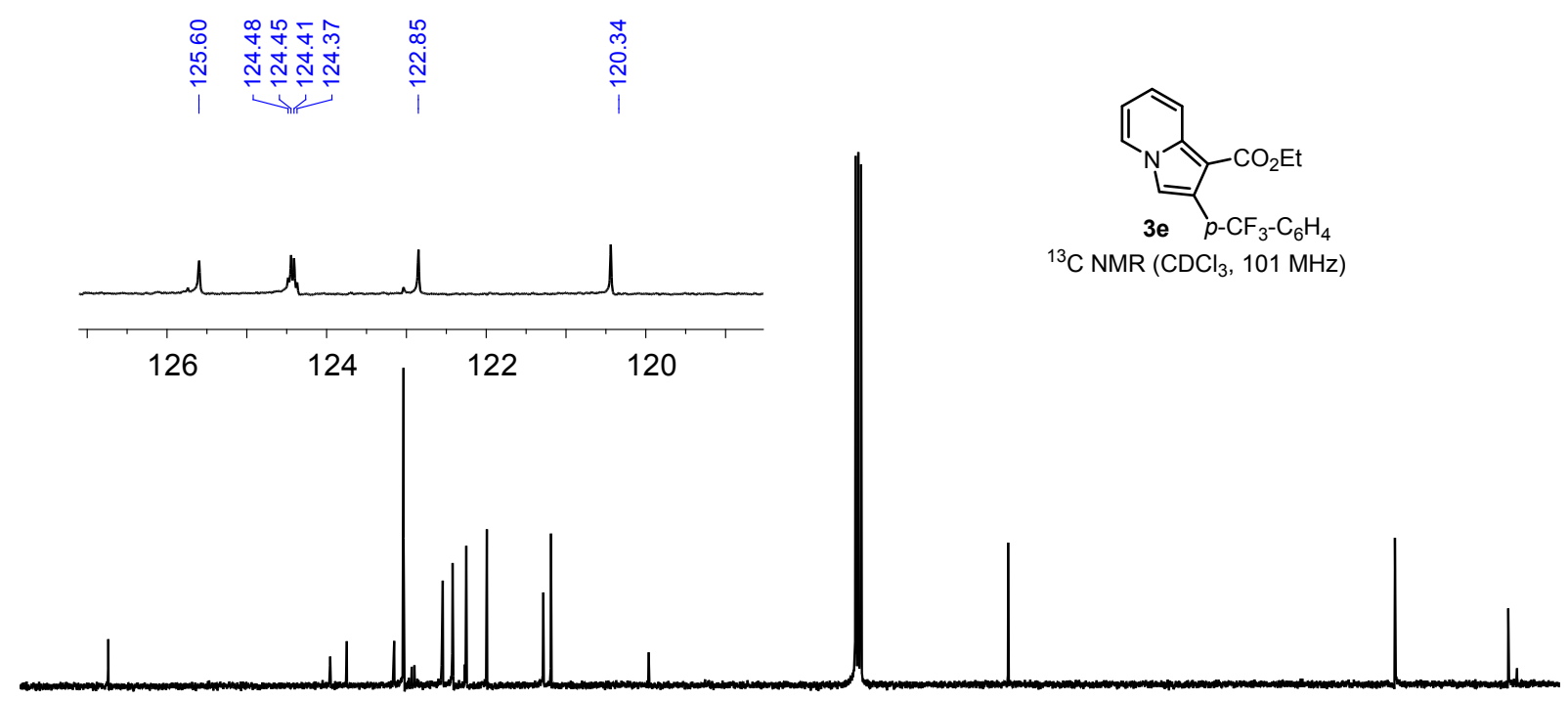

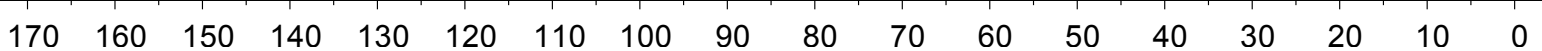




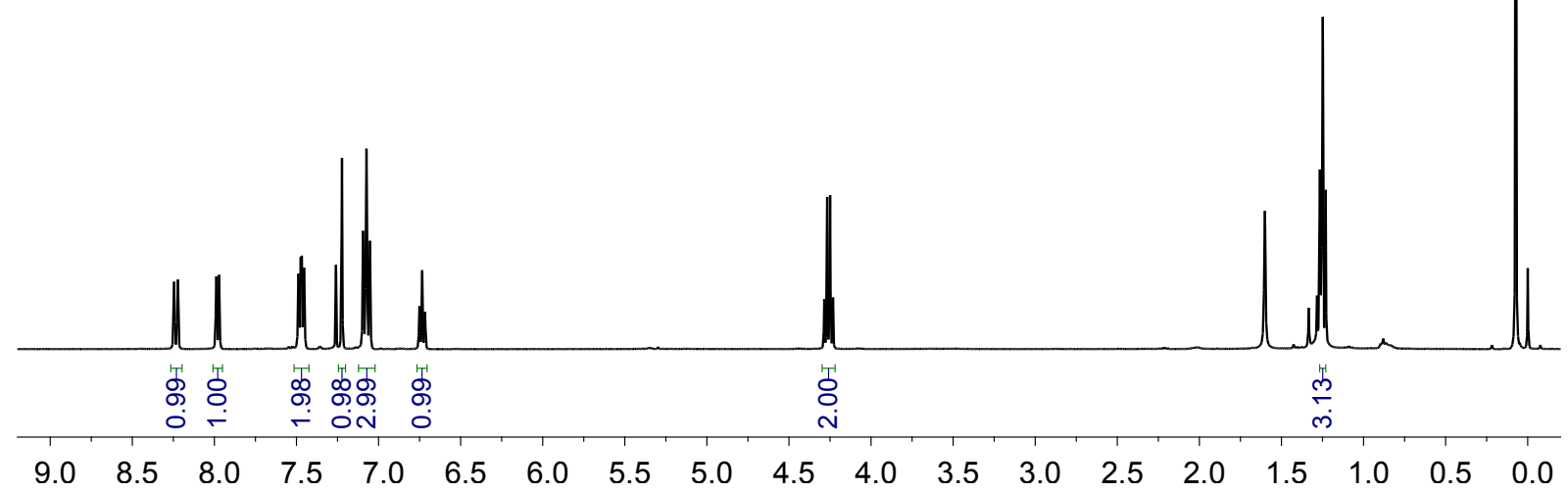

年

i

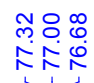

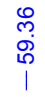

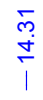
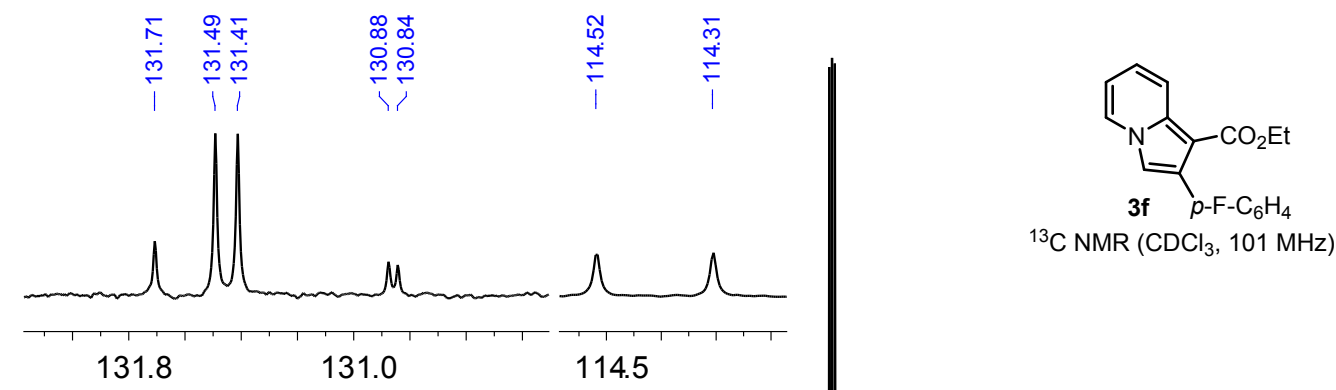

4

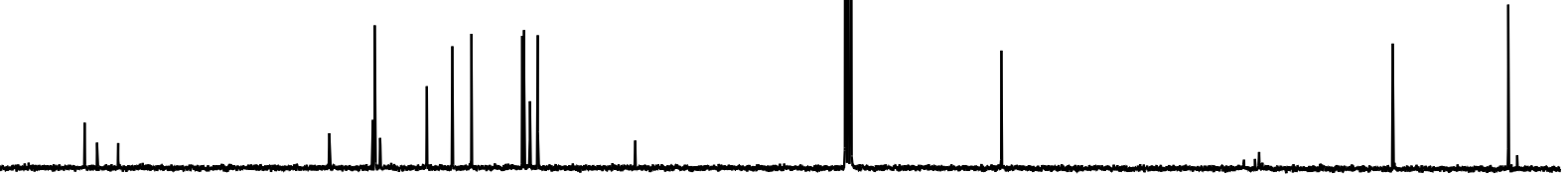

170

160150

$\begin{array}{lllll}40 & 130 & 120 & 110 & 100\end{array}$

$80 \quad 70 \quad 60$

50

40 


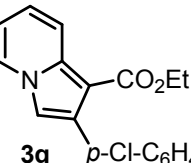

${ }^{1} \mathrm{H} \mathrm{NMR}\left(\mathrm{CDCl}_{3}, 400 \mathrm{MHz}\right)$

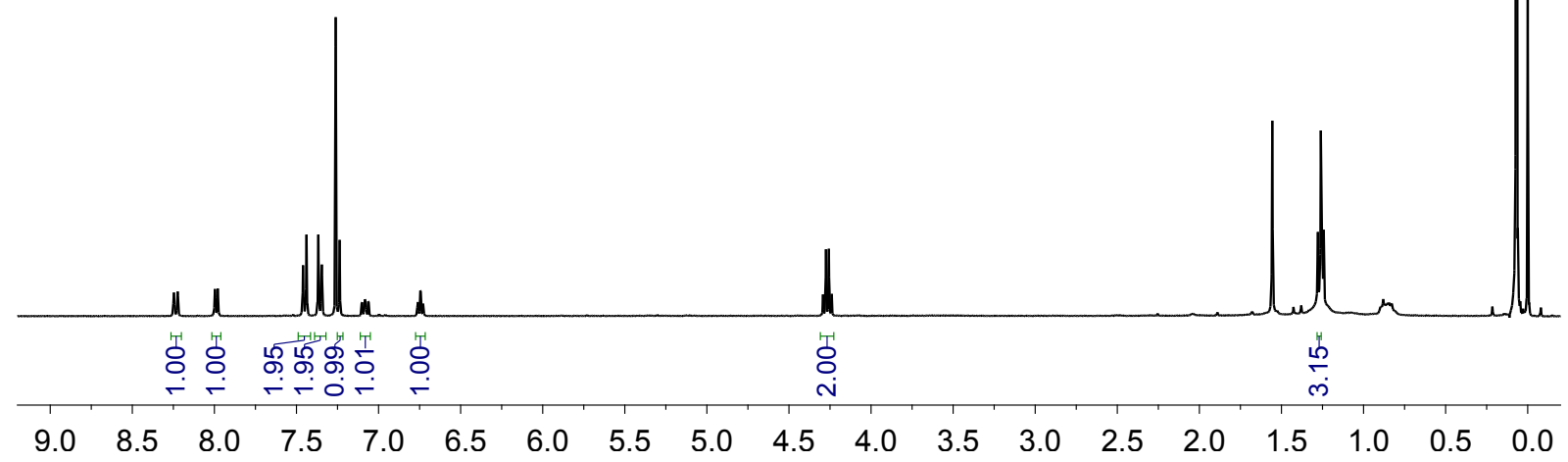

\begin{tabular}{l}
$\stackrel{N}{\infty}$ \\
\multirow{1}{*}{} \\
$\stackrel{0}{1}$
\end{tabular}

ฟั.

$\stackrel{\substack{m \\ \leftarrow}}{\stackrel{m}{+}}$

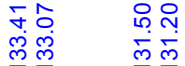

i

$\stackrel{\frac{\pi}{N}}{\frac{1}{i}}$
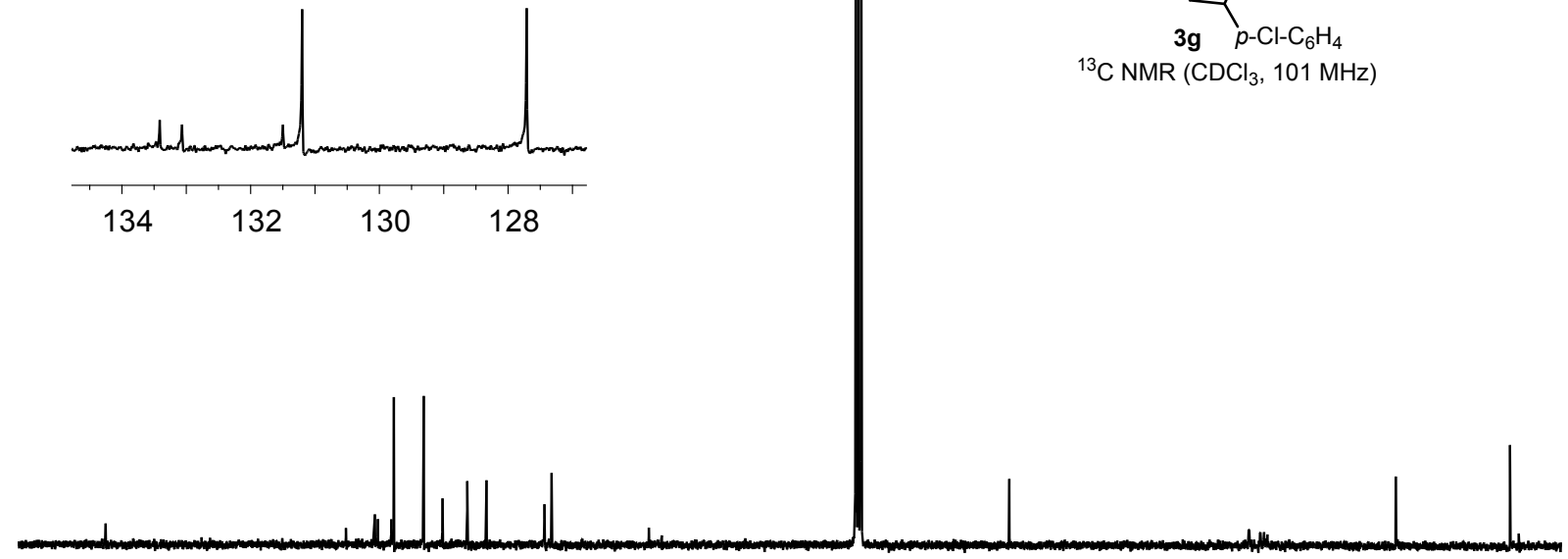

170

160150

$\begin{array}{llll}130 & 120 & 110 & 100\end{array}$

$80 \quad 70 \quad 60$

50

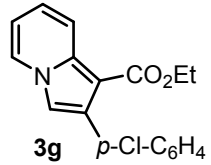

${ }^{13} \mathrm{C} \mathrm{NMR}\left(\mathrm{CDCl}_{3}, 101 \mathrm{MHz}\right)$ 


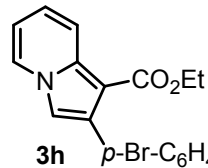

${ }^{1} \mathrm{H} \mathrm{NMR}\left(\mathrm{CDCl}_{3}, 400 \mathrm{MHz}\right)$

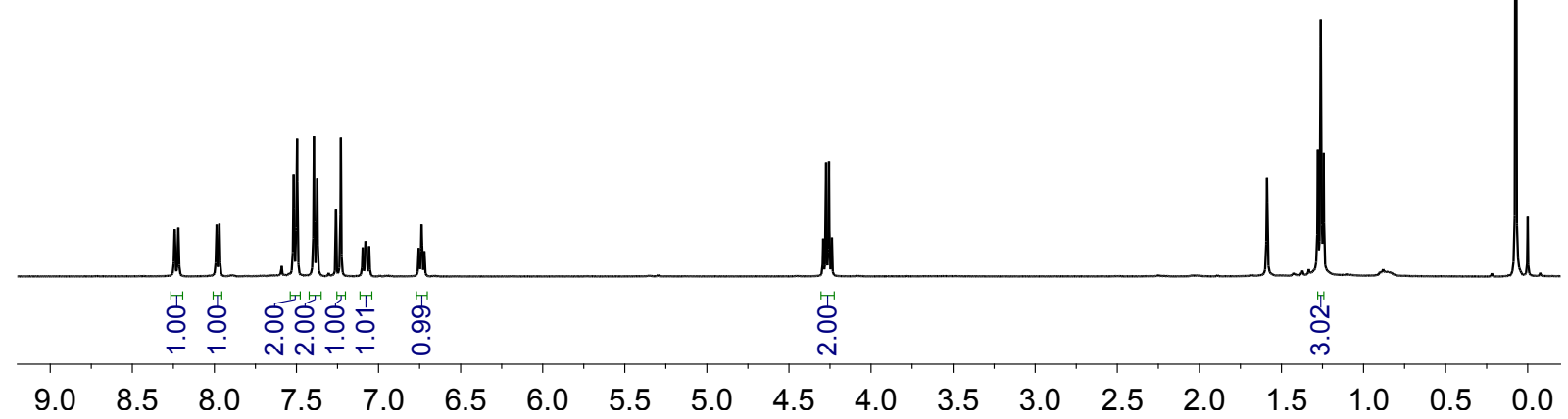

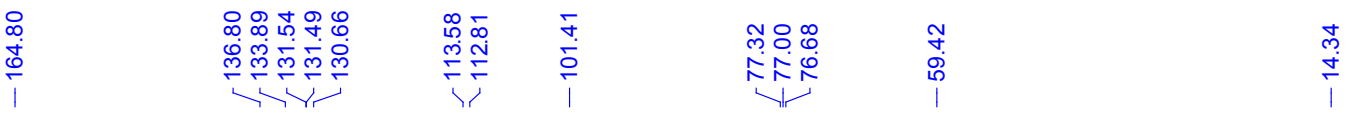
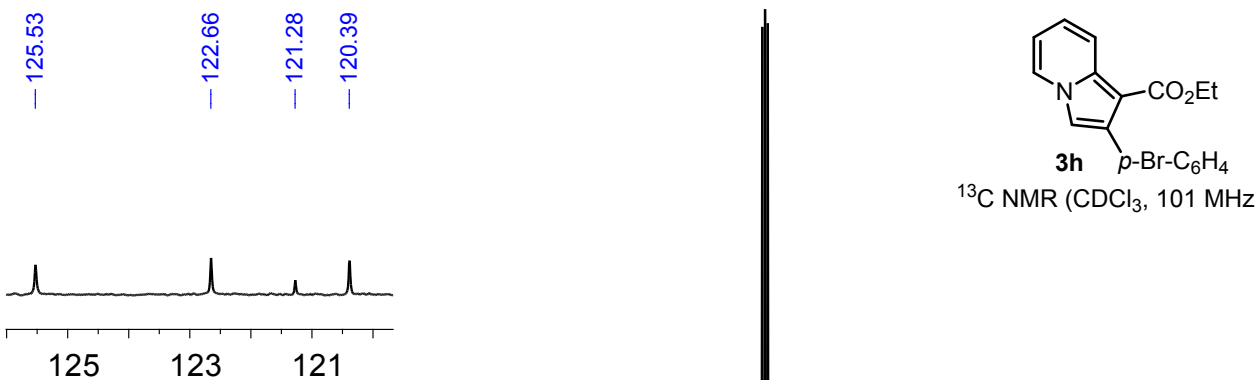

${ }^{13} \mathrm{C} \mathrm{NMR}\left(\mathrm{CDCl}_{3}, 101 \mathrm{MHz}\right)$

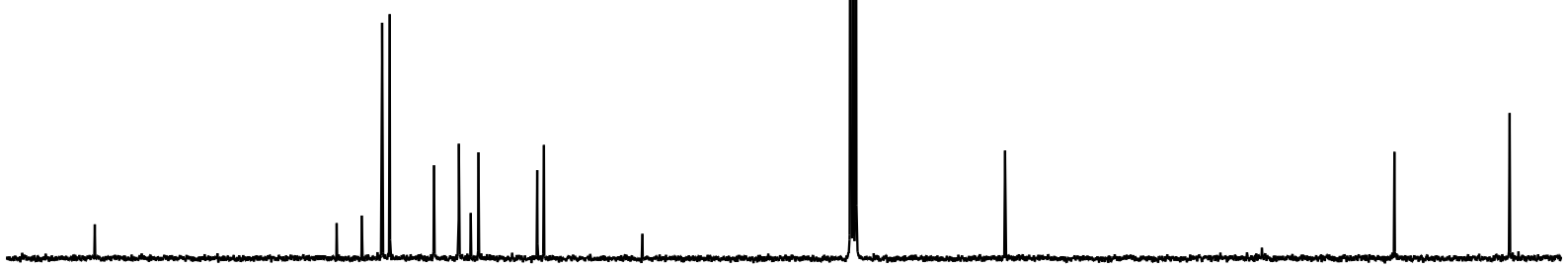

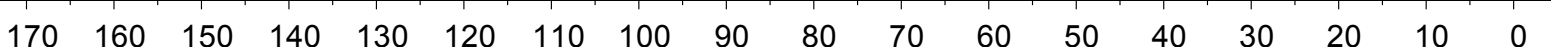




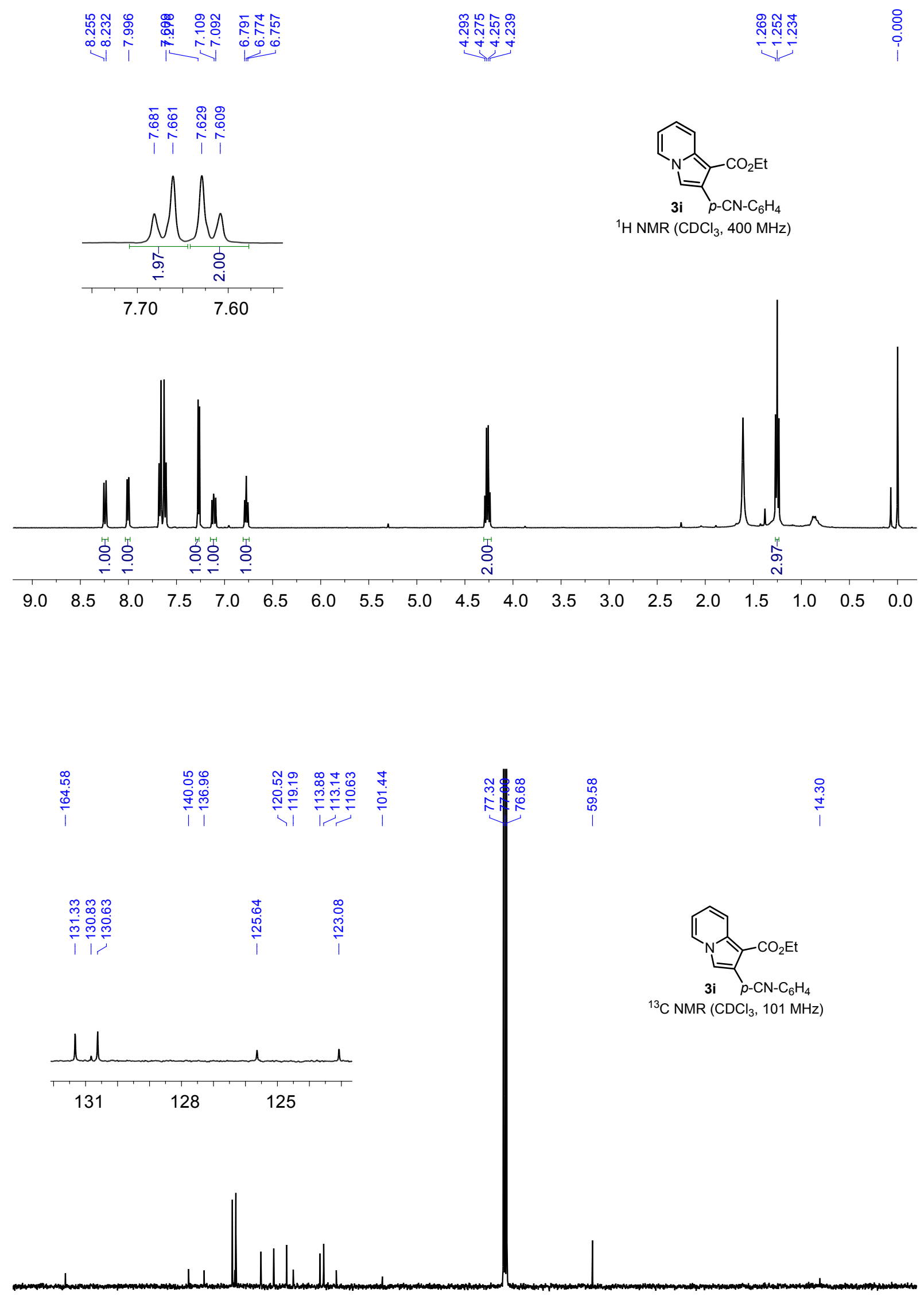

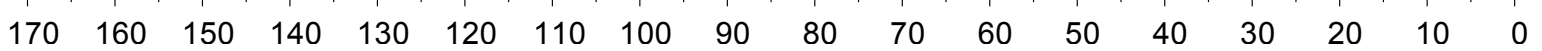




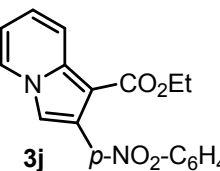

${ }^{1} \mathrm{H} \mathrm{NMR}\left(\mathrm{CDCl}_{3}, 400 \mathrm{MHz}\right)$

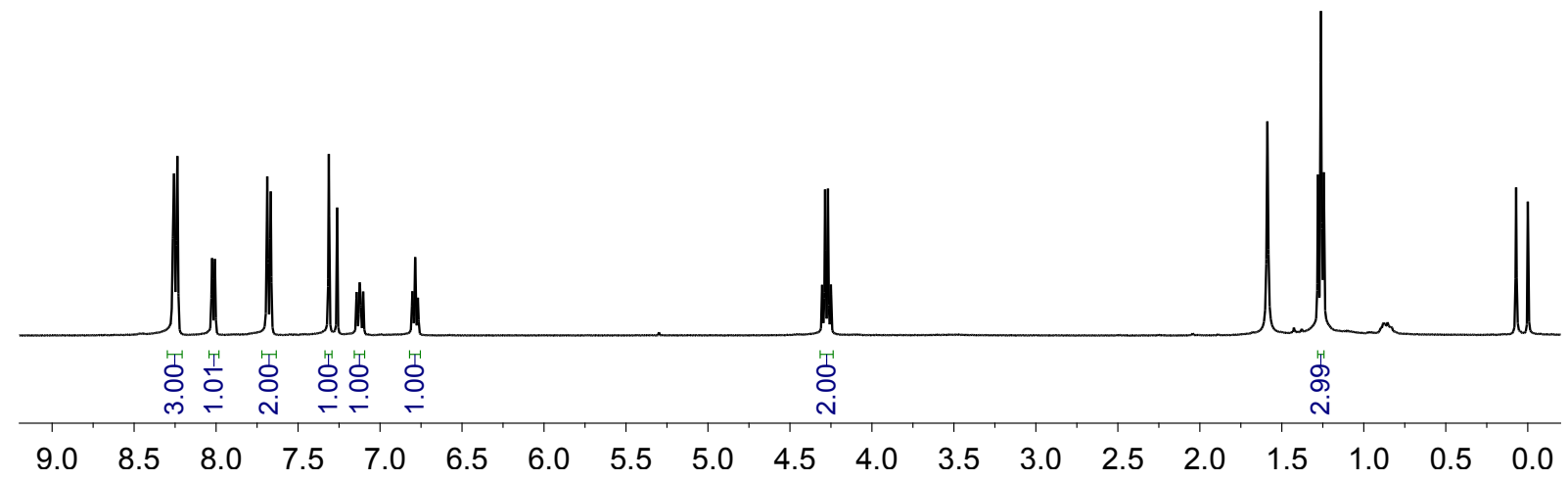

$\begin{array}{llll}1 & \frac{1}{5} & 1\end{array}$

i

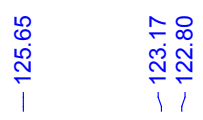

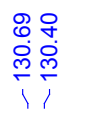
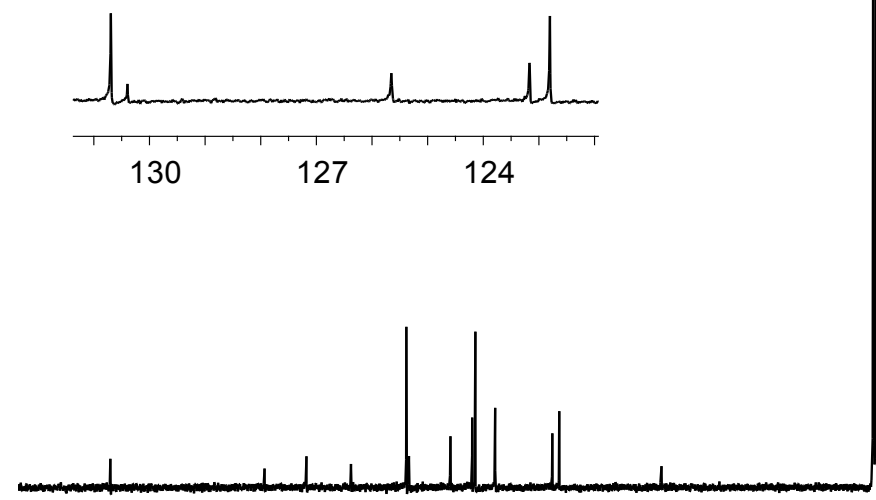

170

160150

140

130120

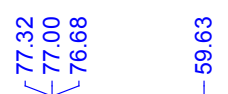

तr

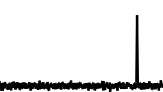

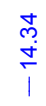

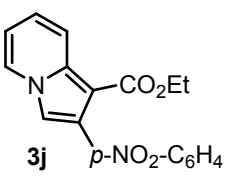

${ }^{13} \mathrm{C} \mathrm{NMR}\left(\mathrm{CDCl}_{3}, 101 \mathrm{MHz}\right)$ 


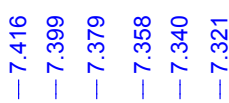

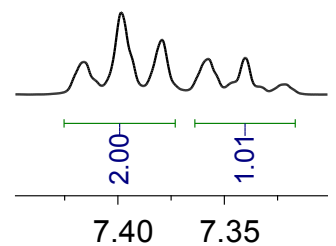

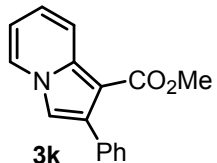

${ }^{1} \mathrm{H} \mathrm{NMR}\left(\mathrm{CDCl}_{3}, 400 \mathrm{MHz}\right)$
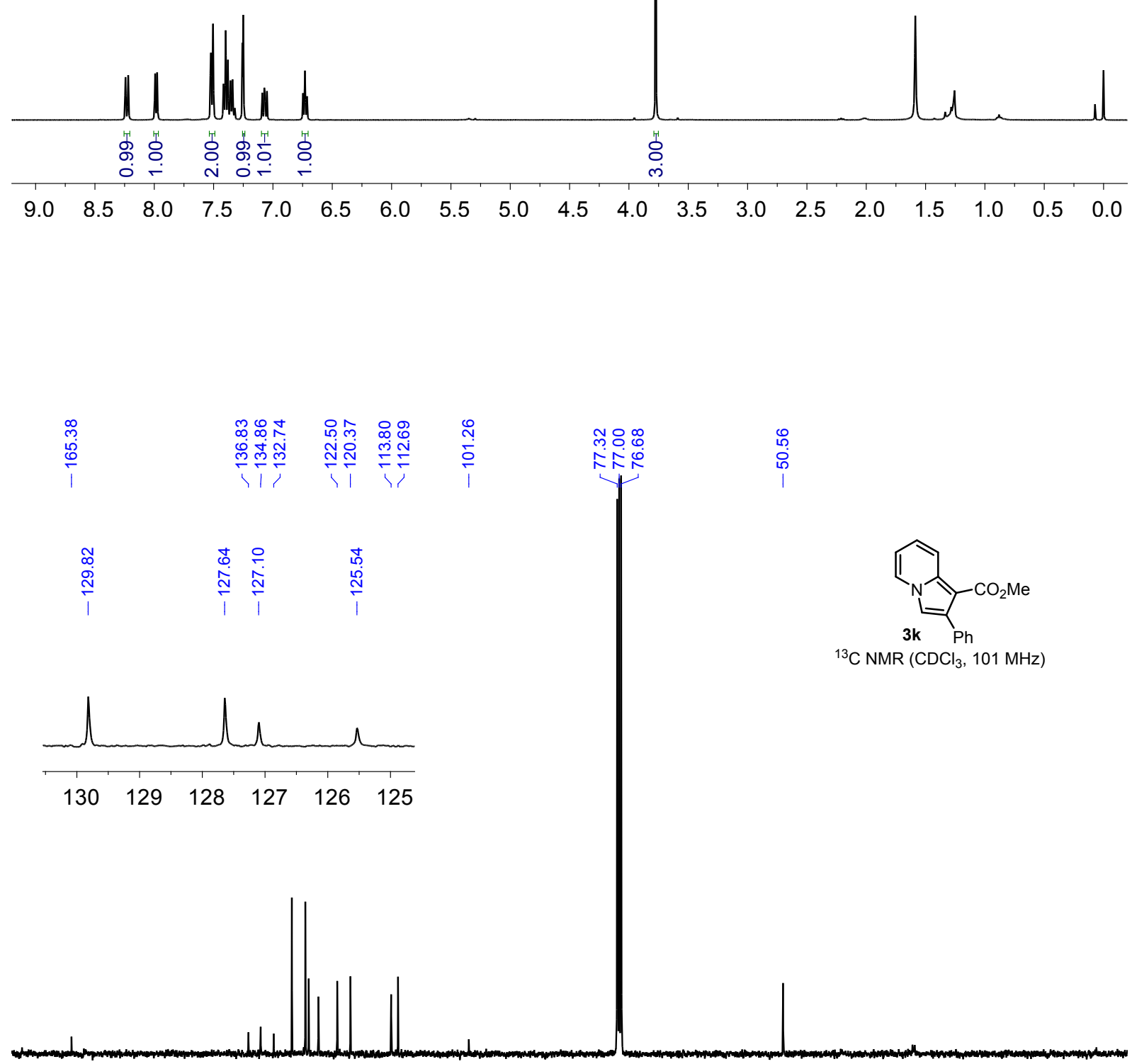

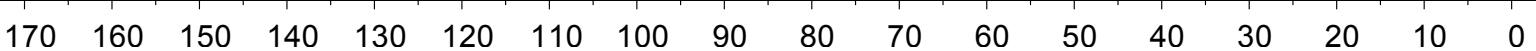




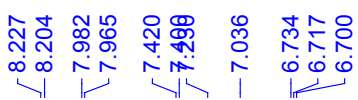

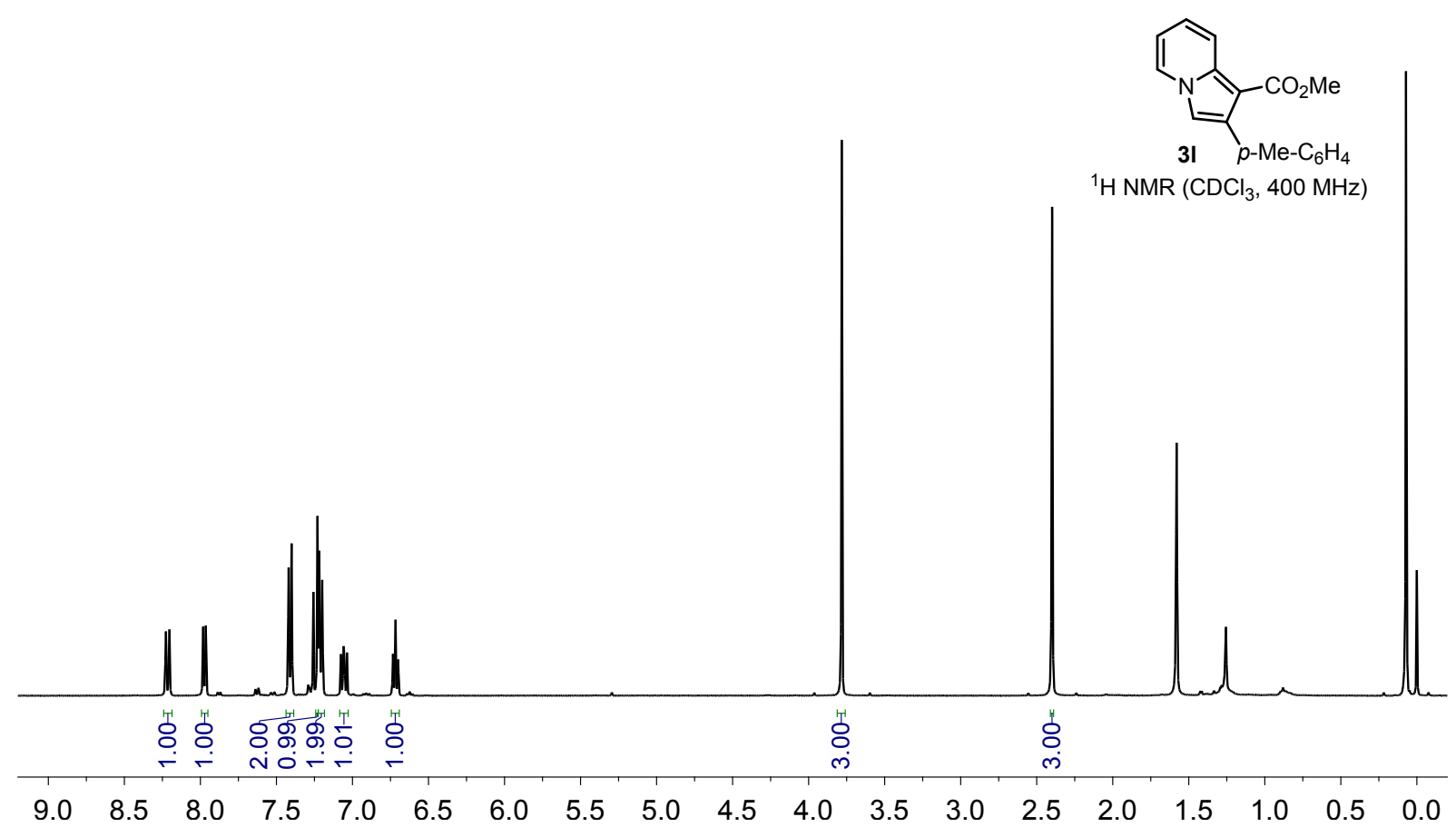

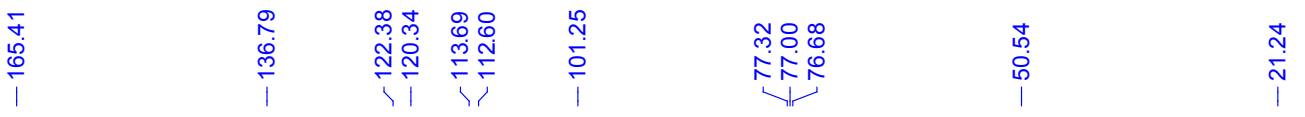

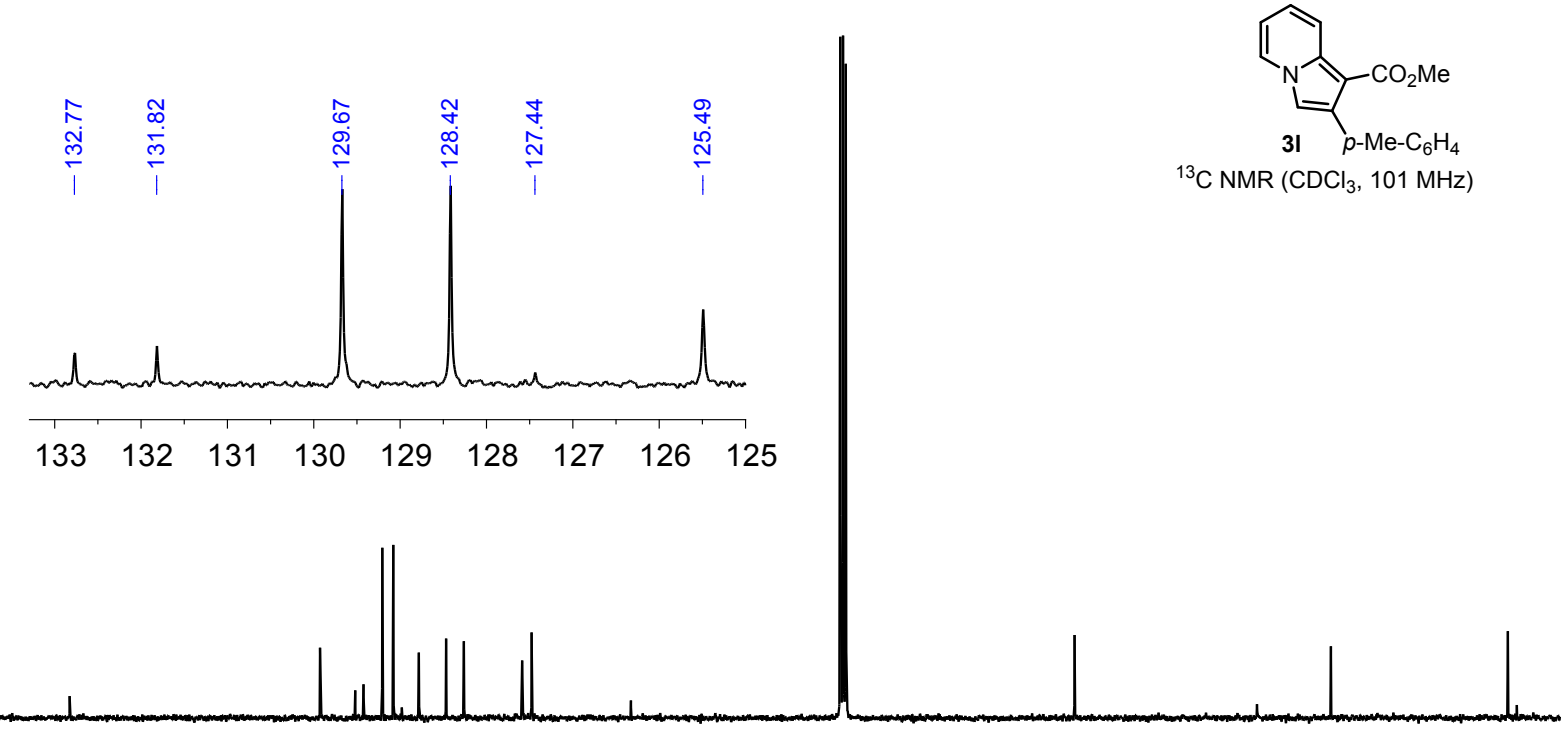

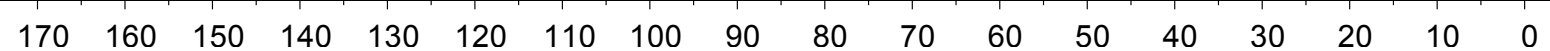




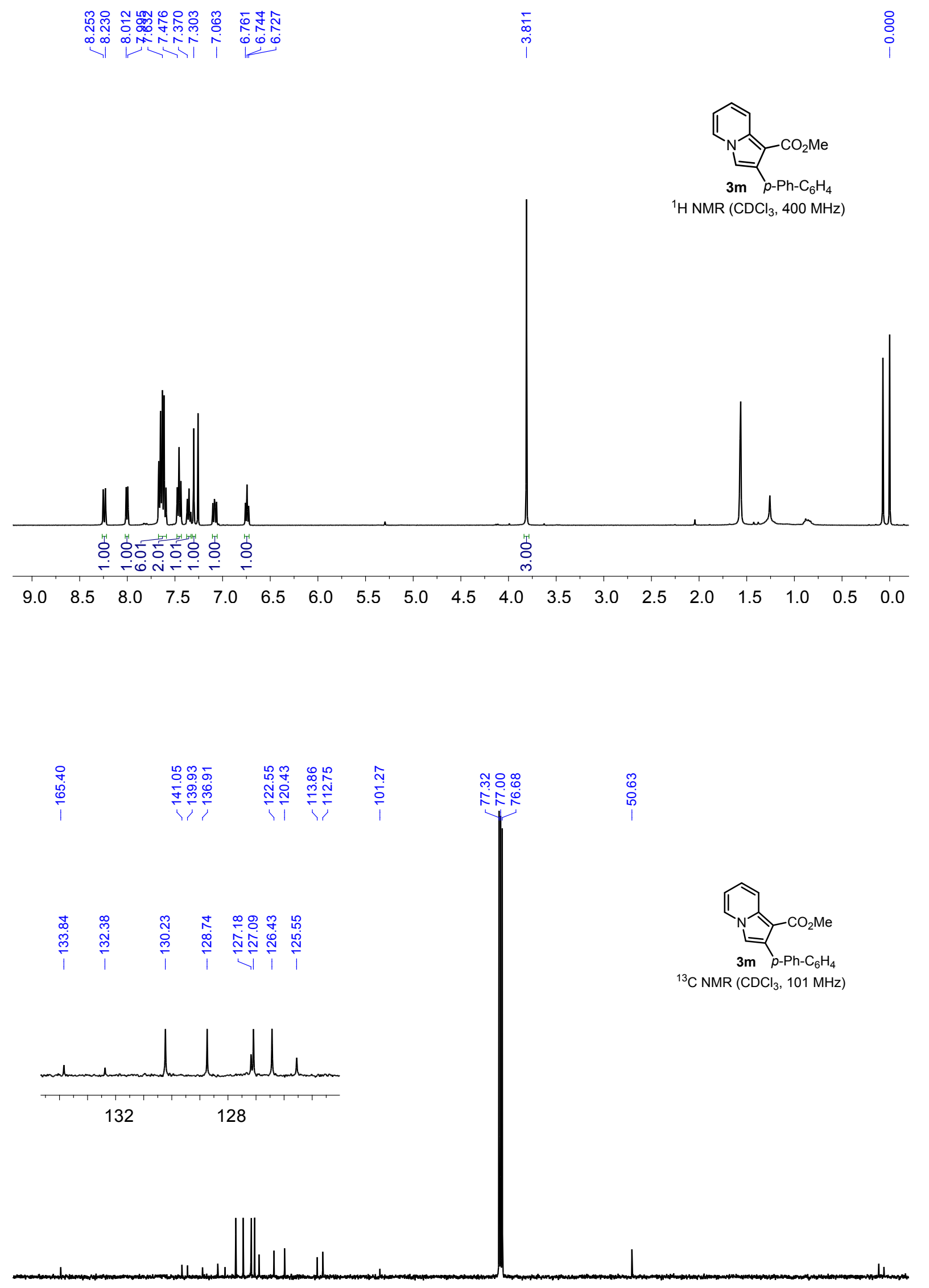

$\begin{array}{llllllllllllllllll}170 & 160 & 150 & 140 & 130 & 120 & 110 & 100 & 90 & 80 & 70 & 60 & 50 & 40 & 30 & 20 & 10 & 0\end{array}$ 


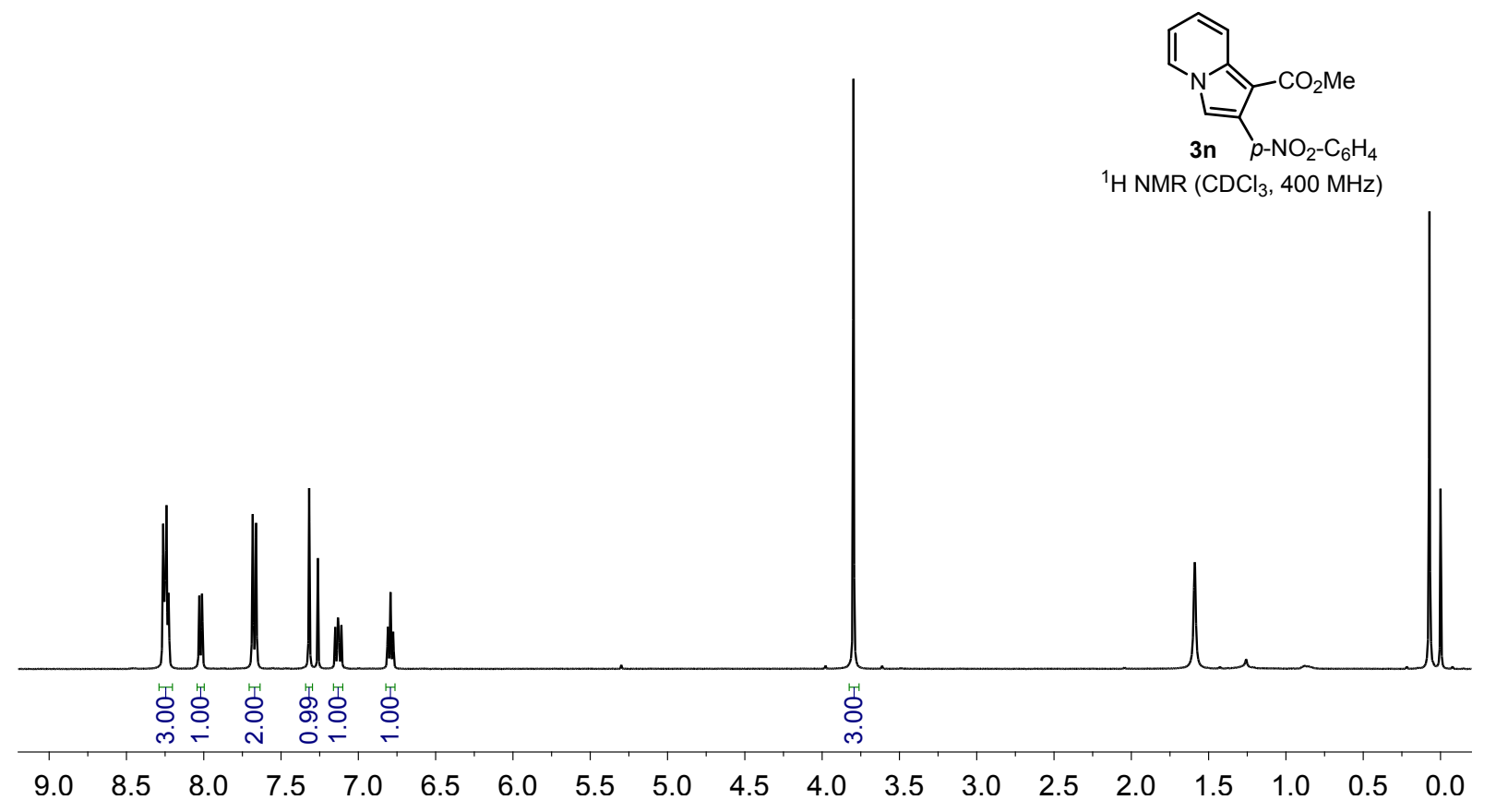

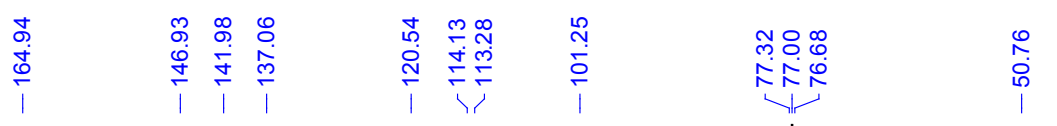
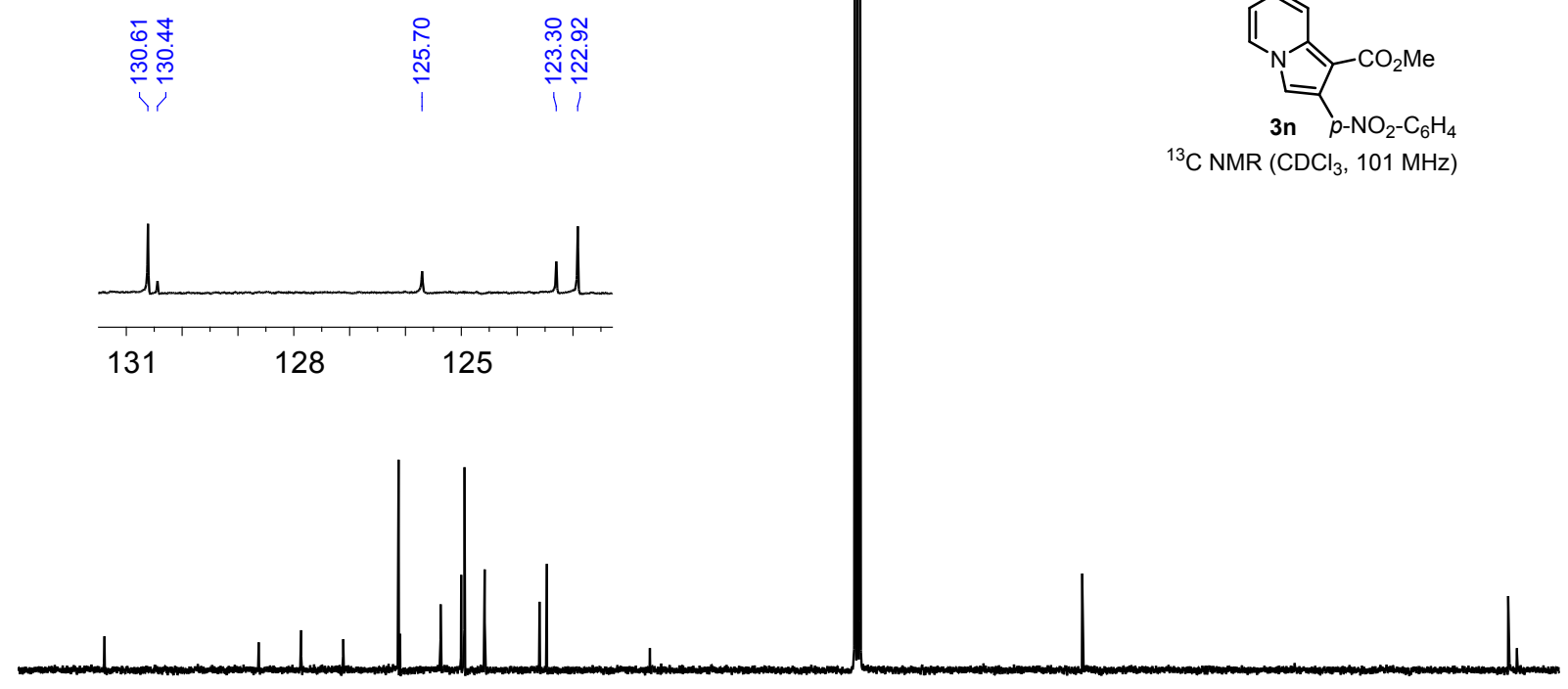

$\begin{array}{llllllllllllllllll}170 & 160 & 150 & 140 & 130 & 120 & 110 & 100 & 90 & 80 & 70 & 60 & 50 & 40 & 30 & 20 & 10 & 0\end{array}$ 


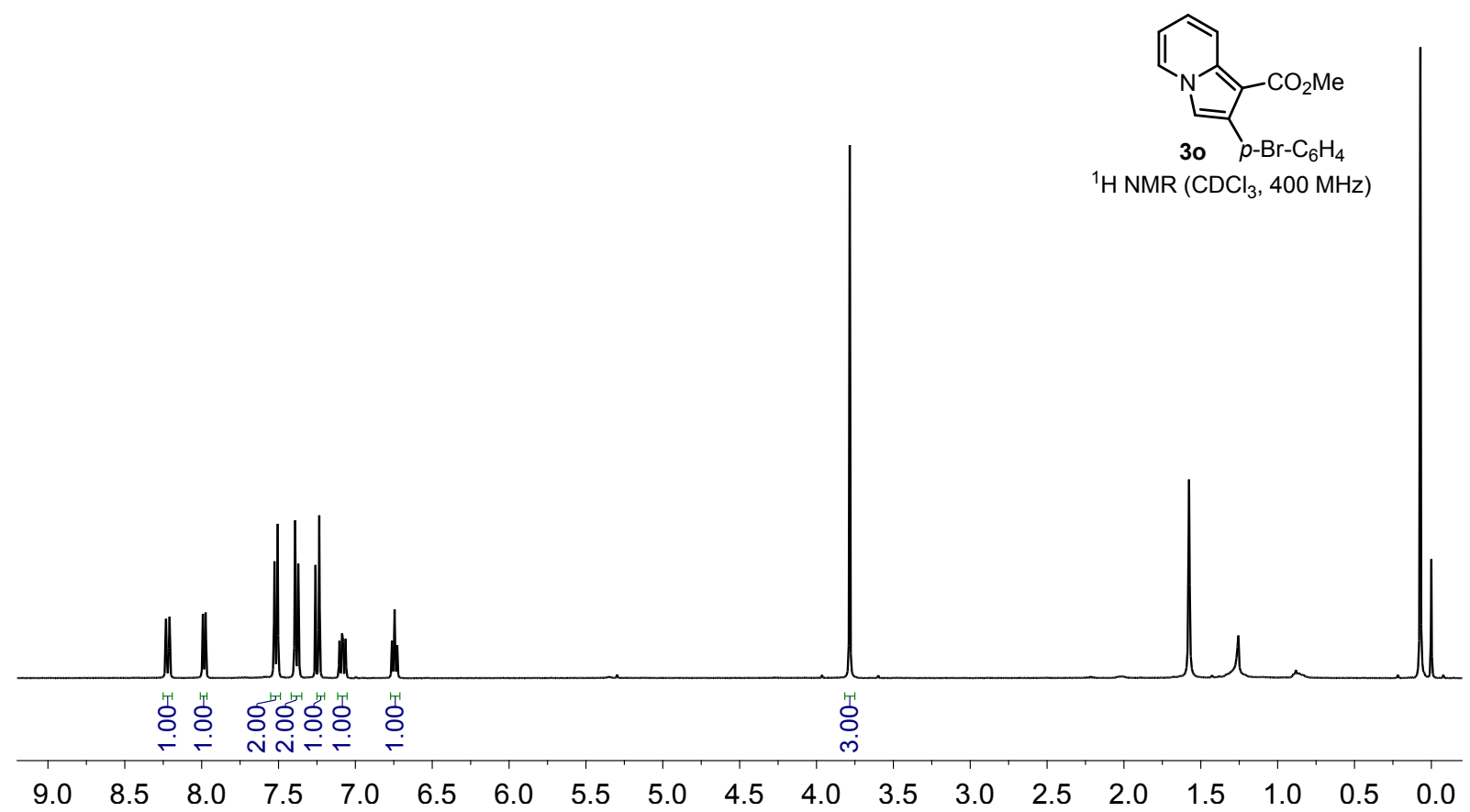

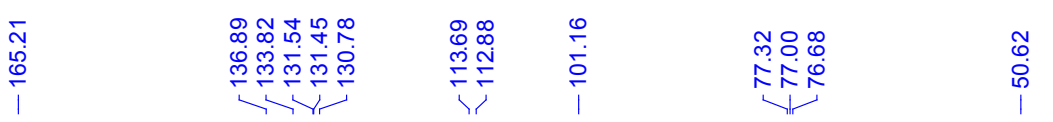
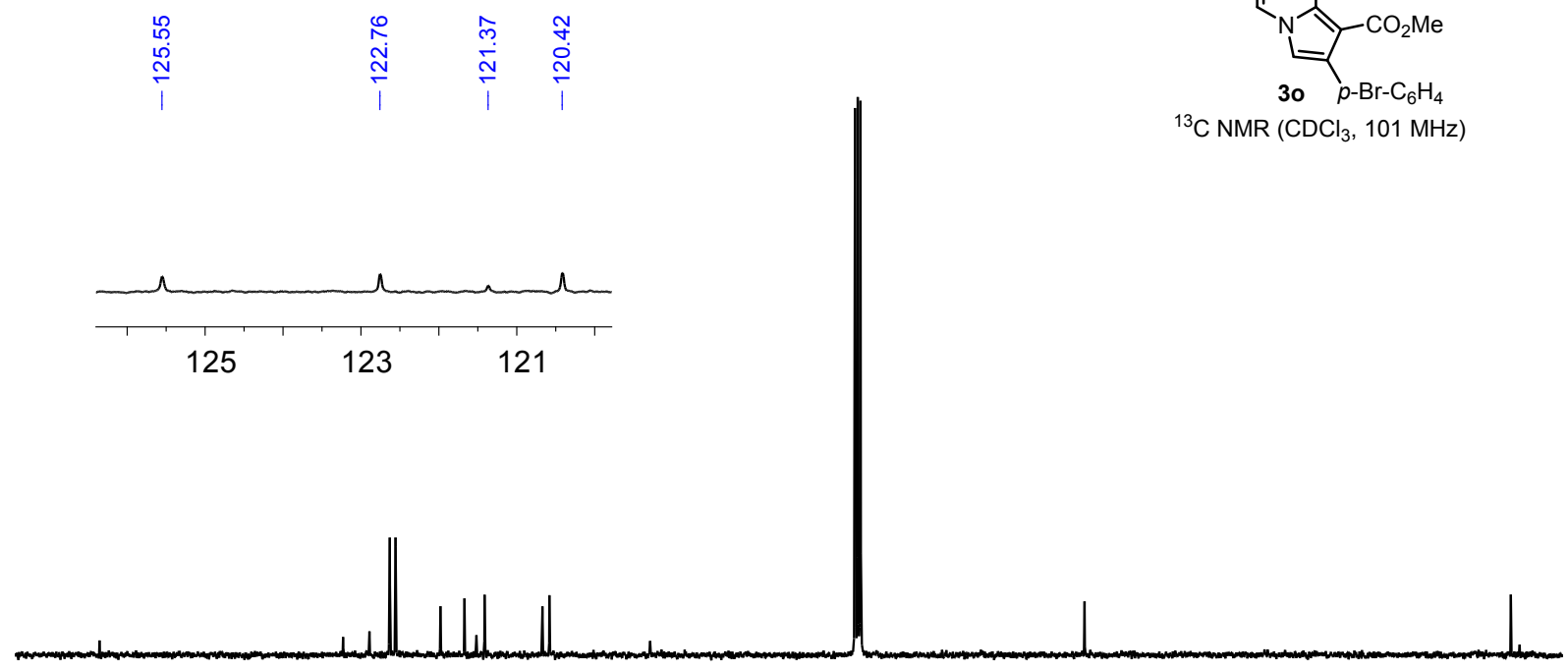

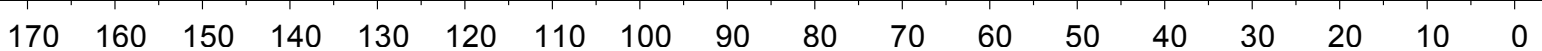




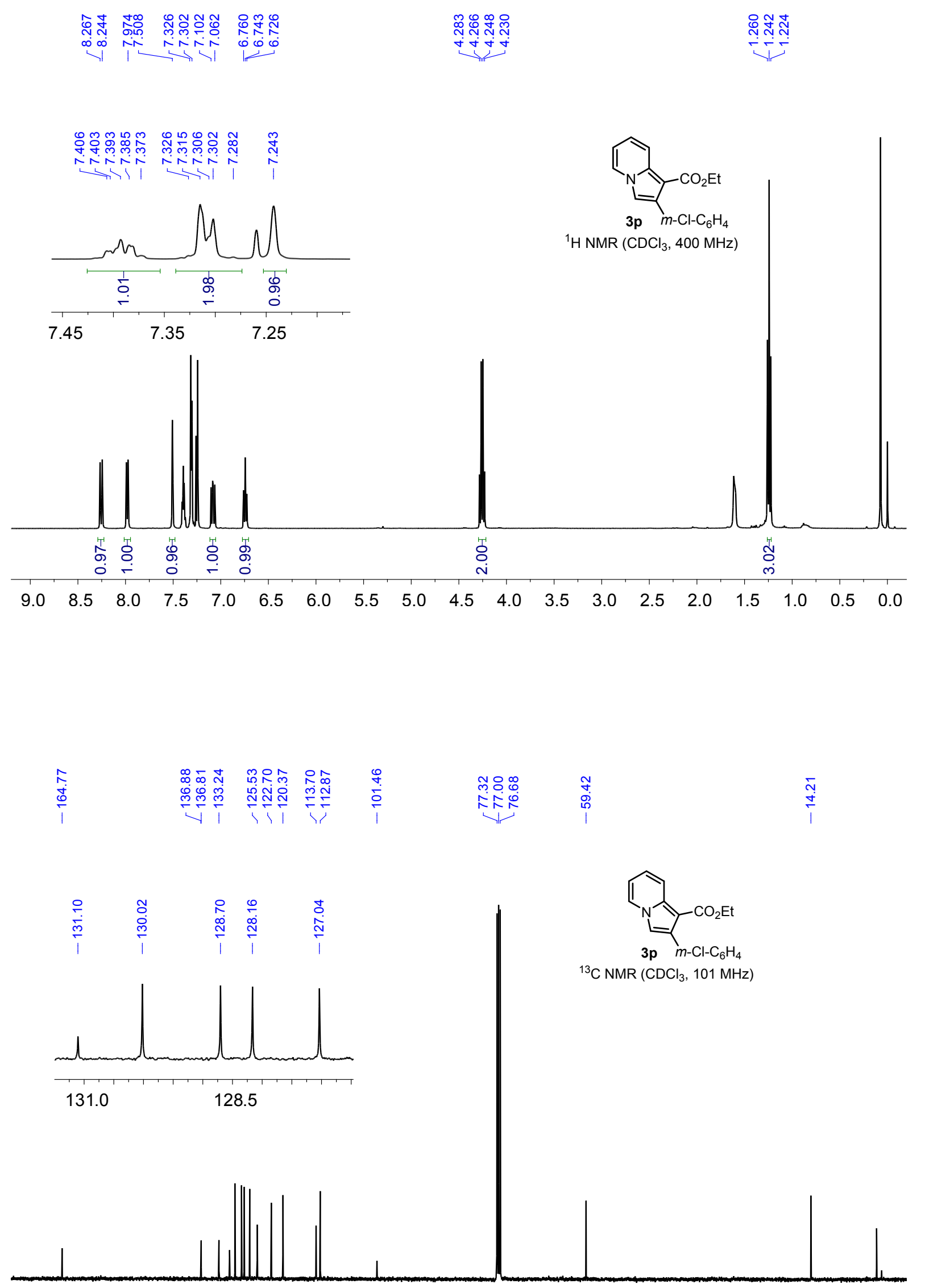

$\begin{array}{llllllllllllllllll}170 & 160 & 150 & 140 & 130 & 120 & 110 & 100 & 90 & 80 & 70 & 60 & 50 & 40 & 30 & 20 & 10 & 0\end{array}$ 


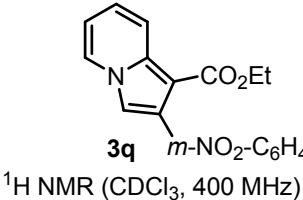

${ }^{1} \mathrm{H} \mathrm{NMR}\left(\mathrm{CDCl}_{3}, 400 \mathrm{MHz}\right)$

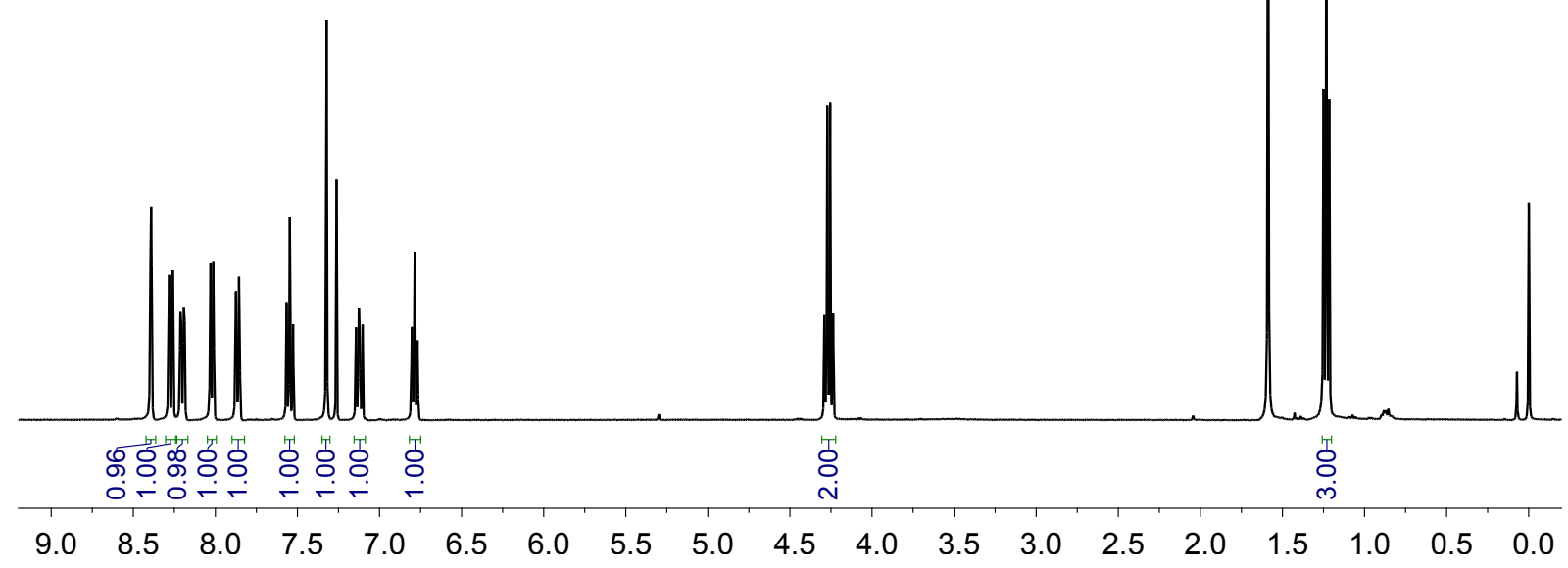

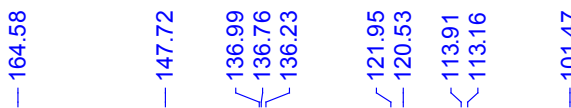

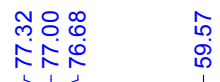

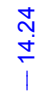

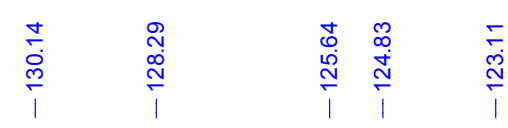
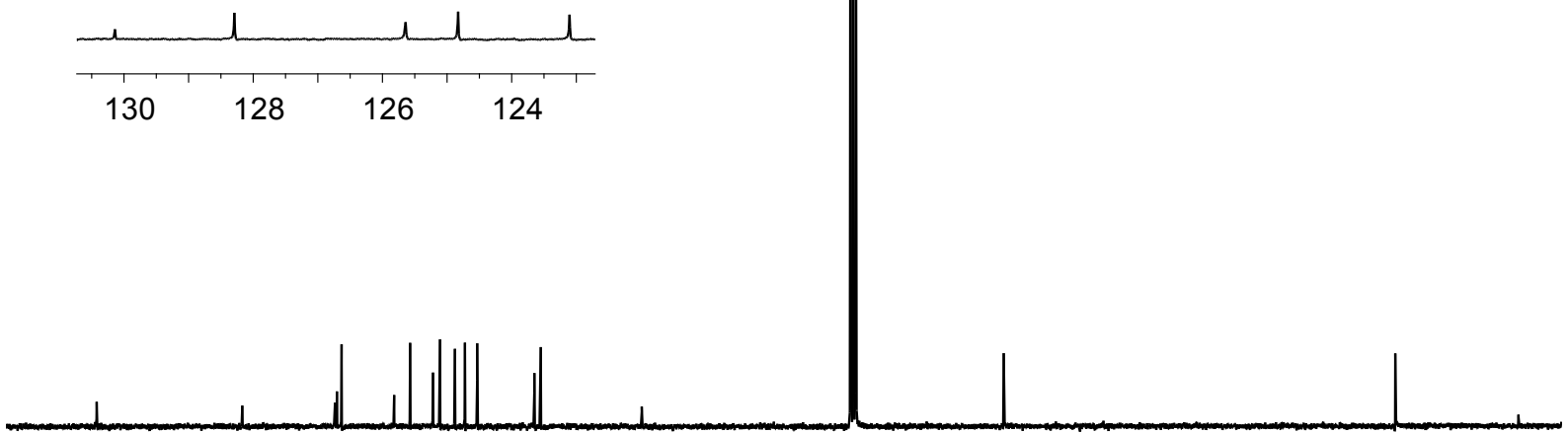

170

160150

1301

$\begin{array}{llll}110 & 100 & 90\end{array}$

$80 \quad 70$

$60 \quad 50$

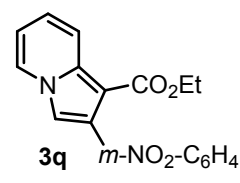

${ }^{13} \mathrm{C} \mathrm{NMR}\left(\mathrm{CDCl}_{3}, 101 \mathrm{MHz}\right)$ 


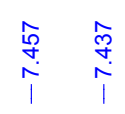
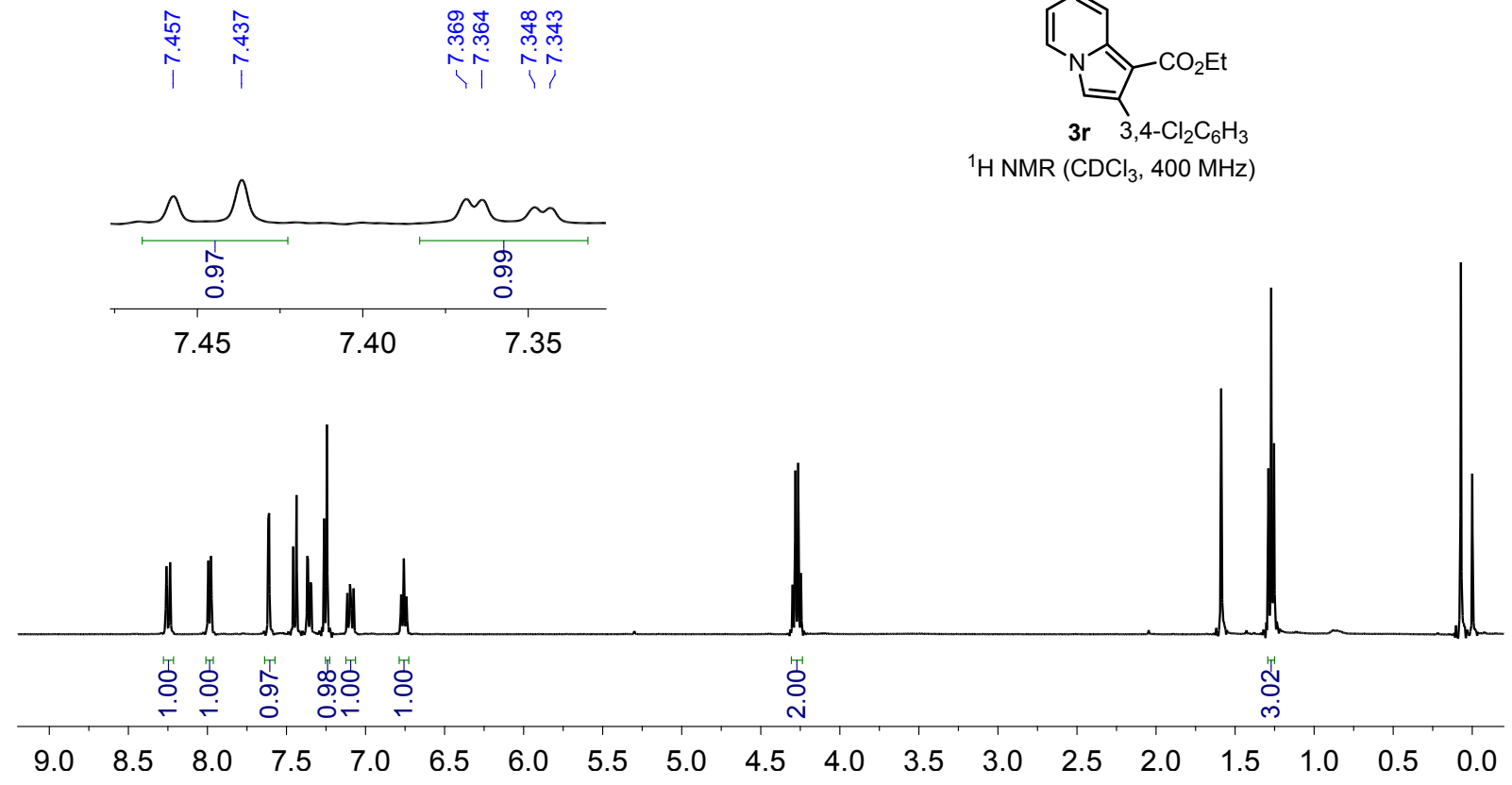

苗
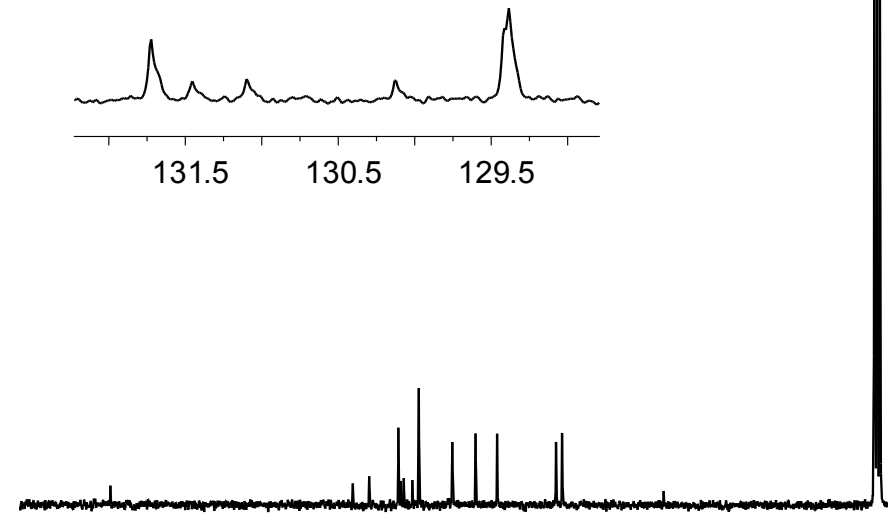

170

160150

140

$\begin{array}{llll}130 & 120 \quad 110 \quad 100\end{array}$

$8070 \quad 60$

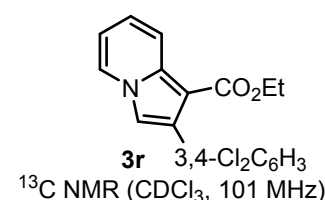

${ }^{1} \mathrm{H} \mathrm{NMR}\left(\mathrm{CDCl}_{3}, 400 \mathrm{MHz}\right)$

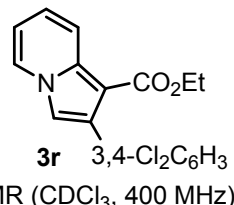

${ }^{13} \mathrm{C} \mathrm{NMR}\left(\mathrm{CDCl}_{3}, 101 \mathrm{MHz}\right)$ 

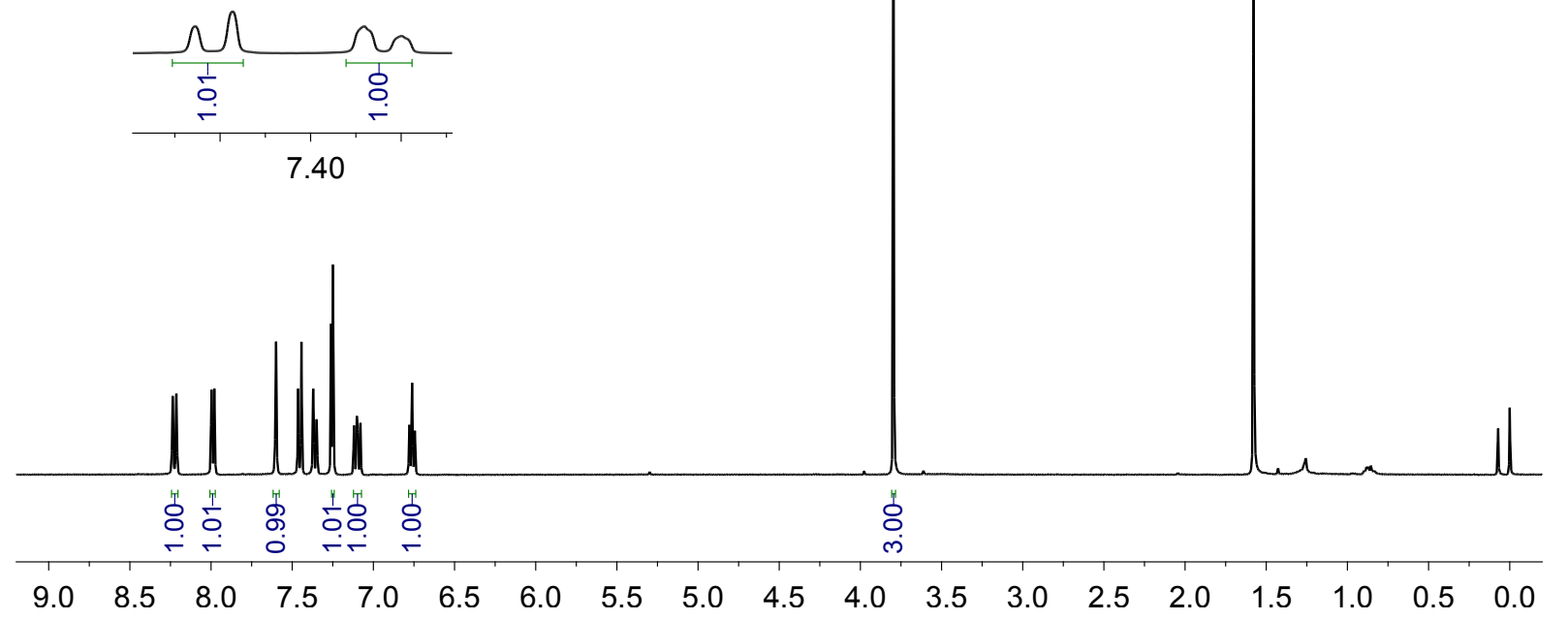

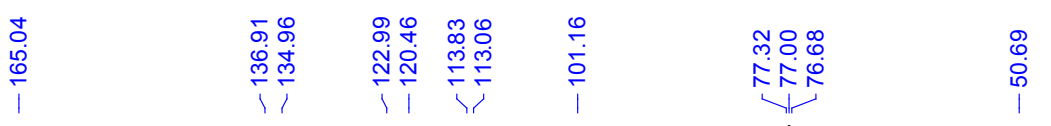
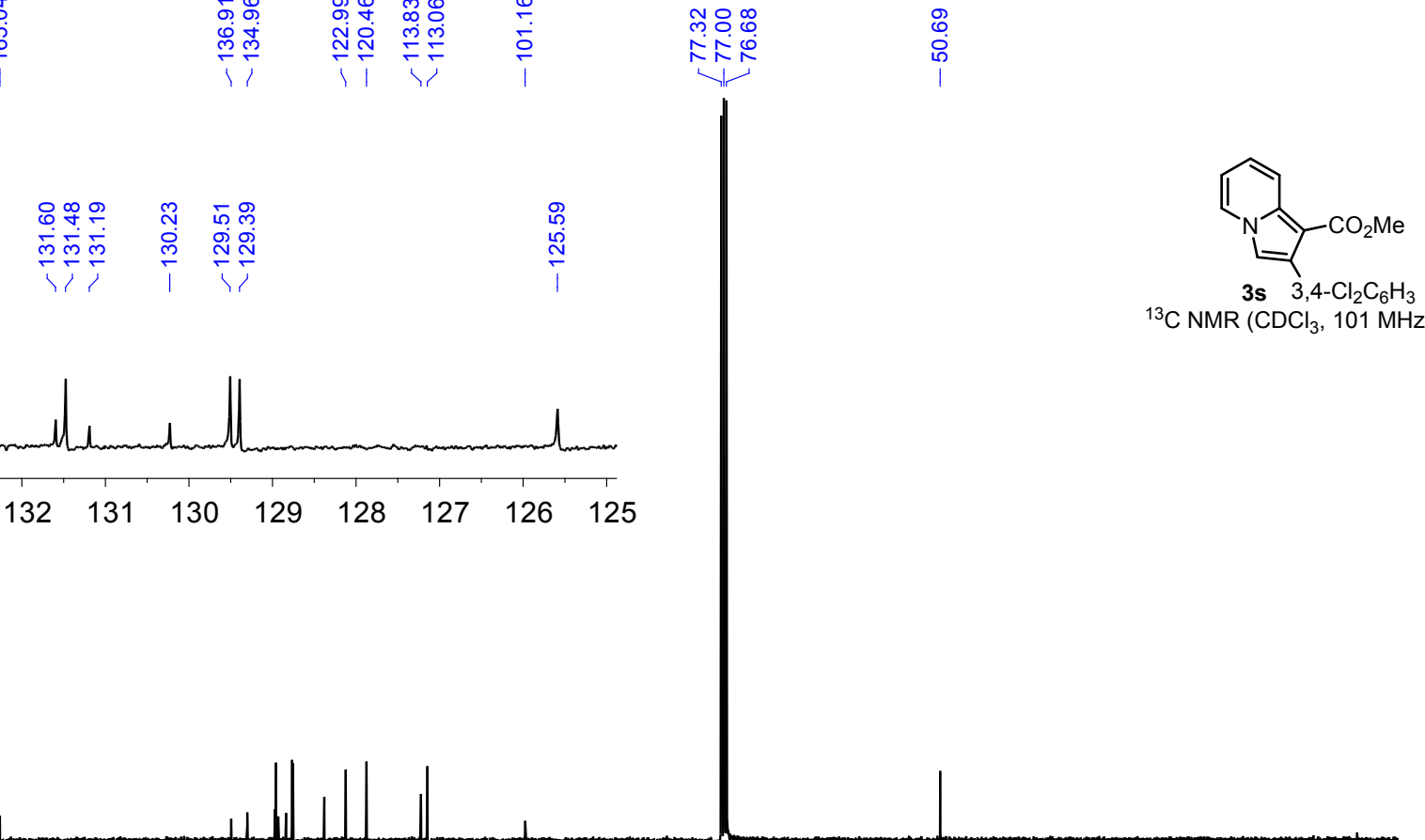

$\begin{array}{llllllllllllllllll}170 & 160 & 150 & 140 & 130 & 120 & 110 & 100 & 90 & 80 & 70 & 60 & 50 & 40 & 30 & 20 & 10 & 0\end{array}$ 


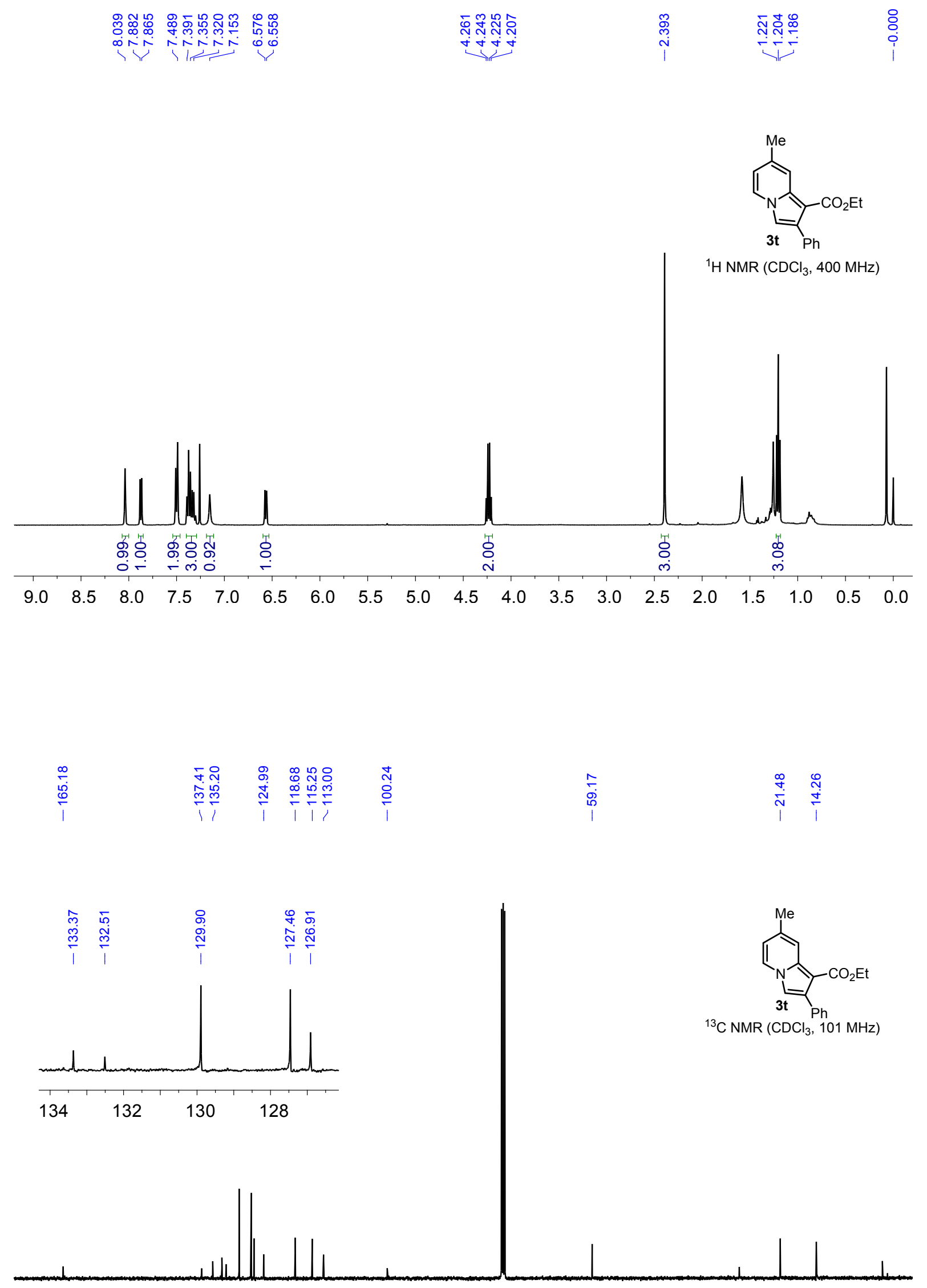

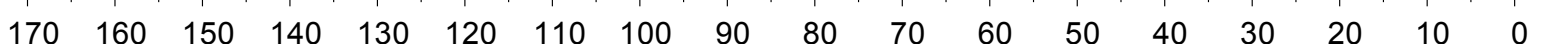




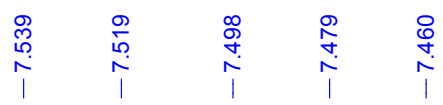

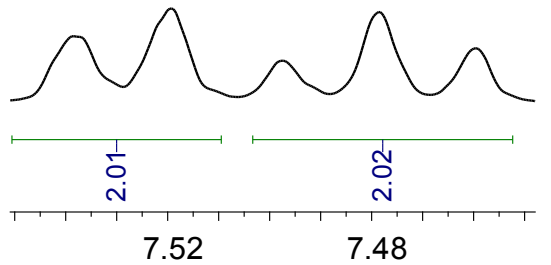

${ }^{1} \mathrm{H} \mathrm{NMR}\left(\mathrm{CDCl}_{3}, 400 \mathrm{MHz}\right)$
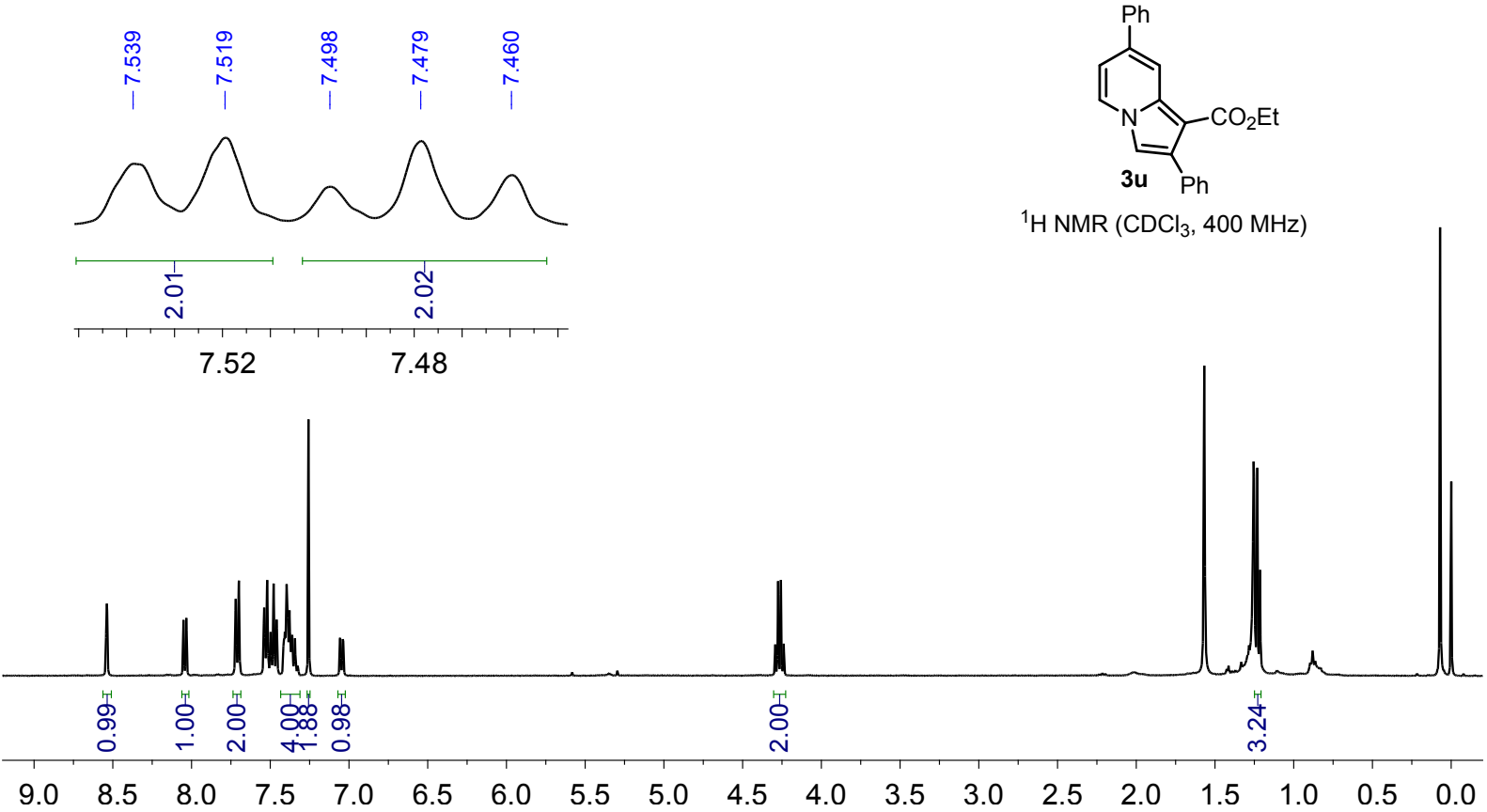

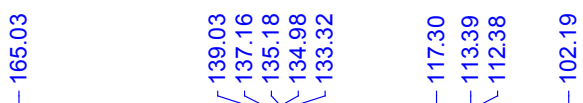

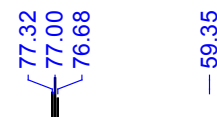

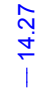

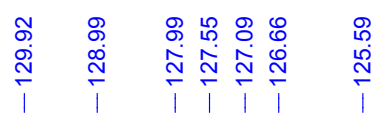

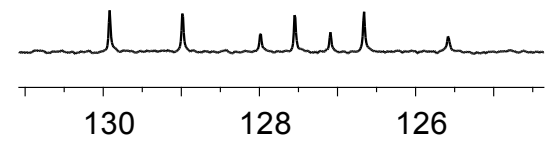

${ }^{13} \mathrm{C} \mathrm{NMR}\left(\mathrm{CDCl}_{3}, 101 \mathrm{MHz}\right)$
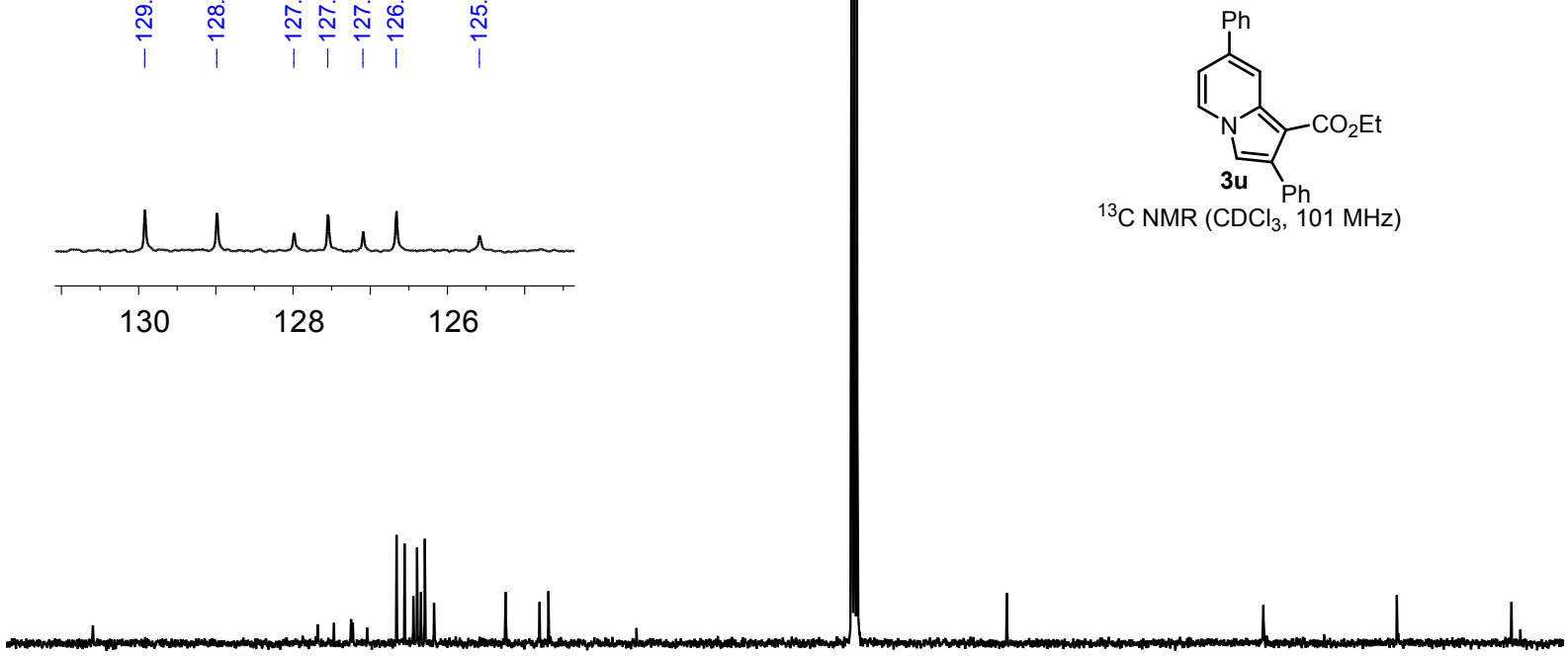

170160

$\begin{array}{llllllll}150 & 140 & 130 & 120 & 110 & 100 & 90\end{array}$

$8070 \quad 60$

$60 \quad 50$

40 


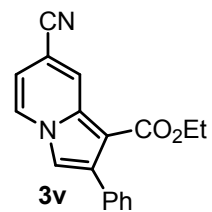

${ }^{1} \mathrm{H} \mathrm{NMR}\left(\mathrm{CDCl}_{3}, 400 \mathrm{MHz}\right)$
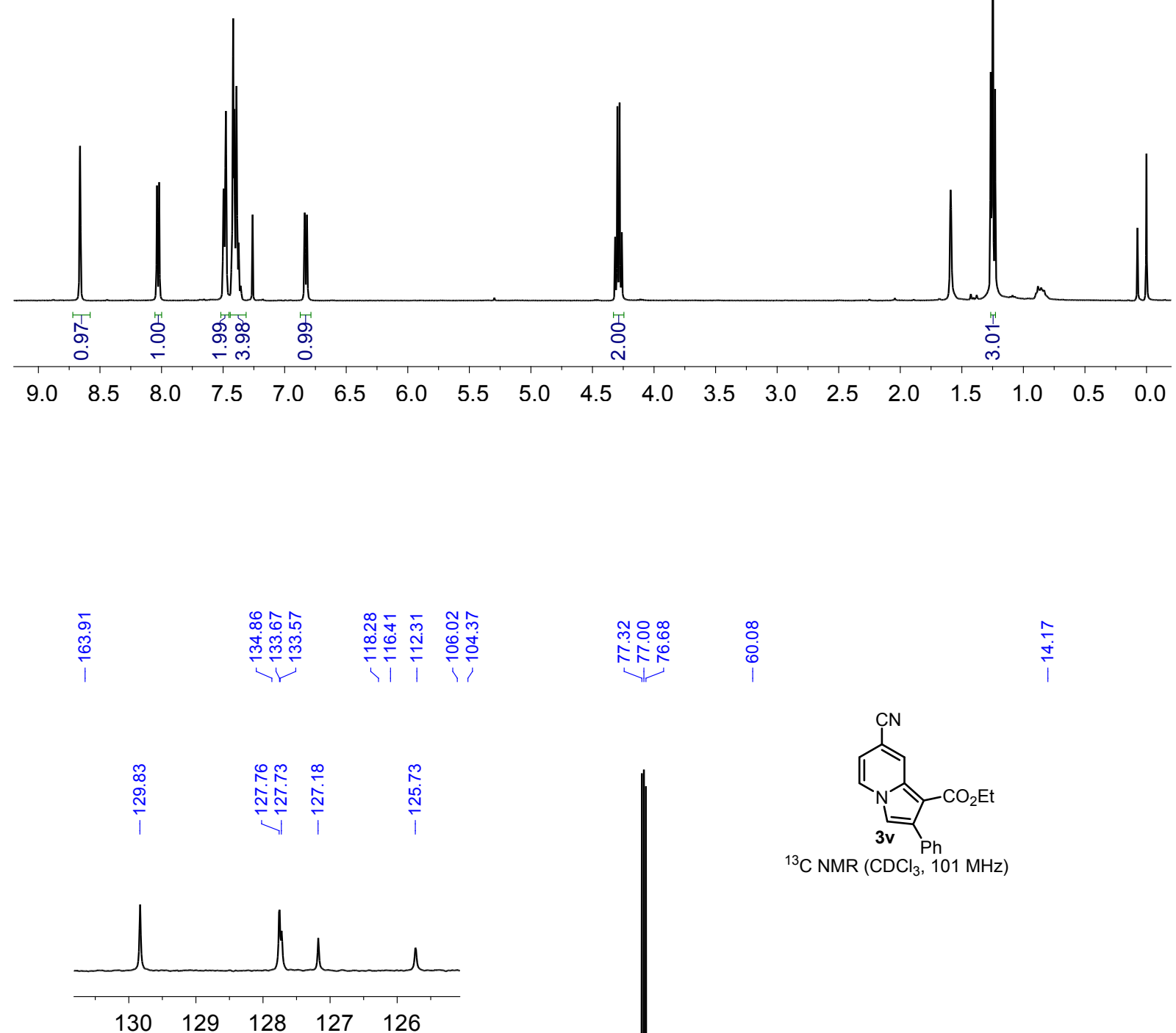

${ }^{13} \mathrm{C} \mathrm{NMR}\left(\mathrm{CDCl}_{3}, 101 \mathrm{MHz}\right)$

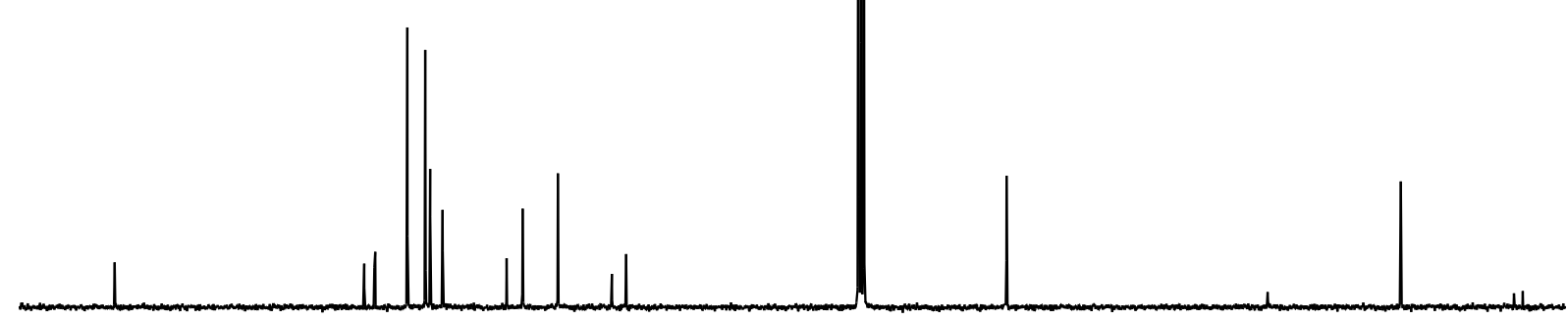

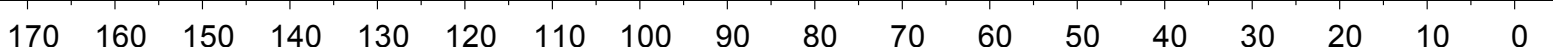




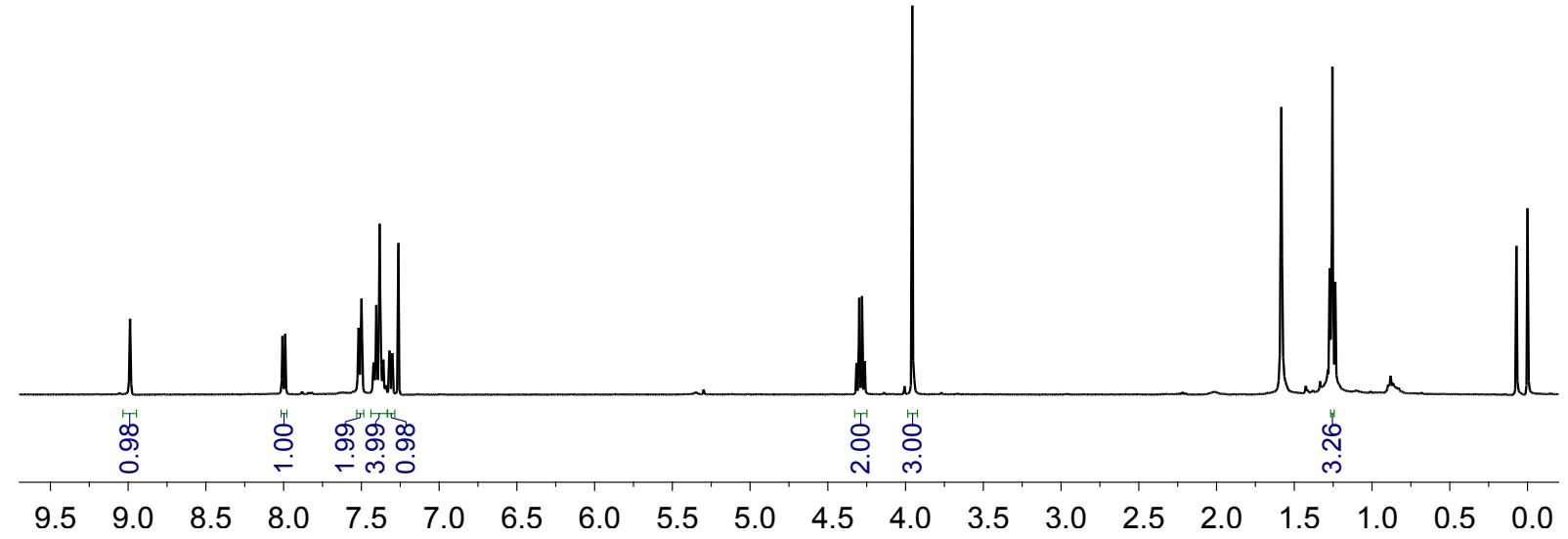

部

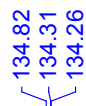

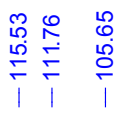

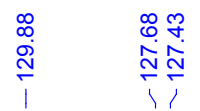

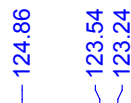
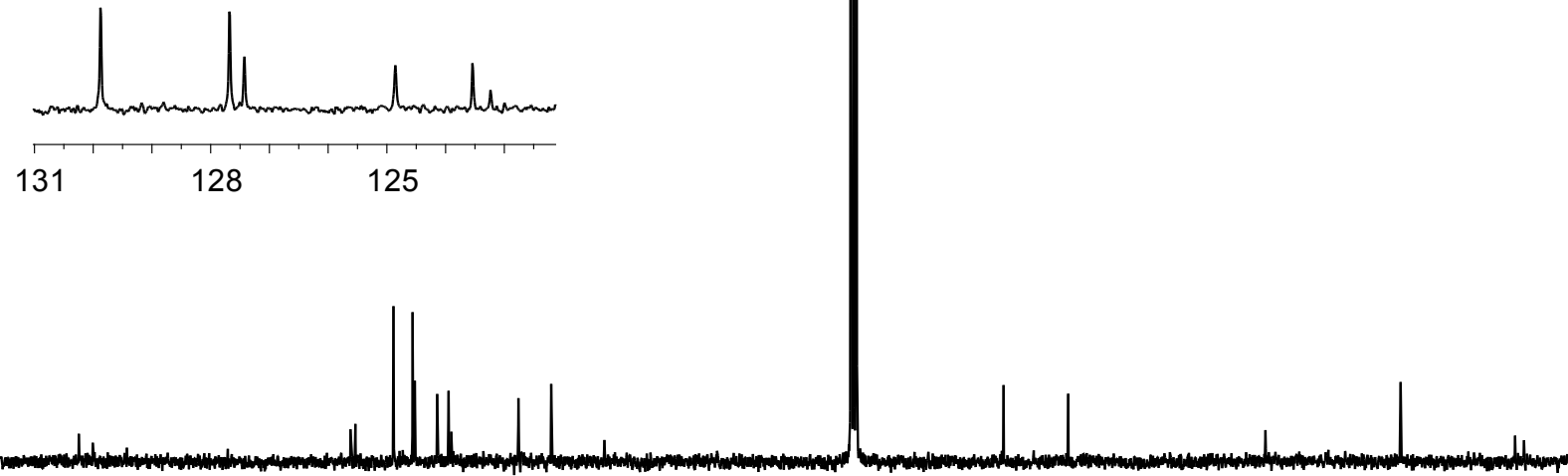

$\begin{array}{llllllllllllllllll}170 & 160 & 150 & 140 & 130 & 120 & 110 & 100 & 90 & 80 & 70 & 60 & 50 & 40 & 30 & 20 & 10 & 0\end{array}$ 


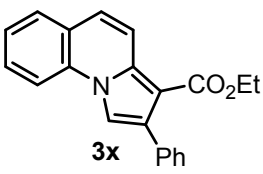

${ }^{1} \mathrm{H}$ NMR $\left(\mathrm{CDCl}_{3}, 400 \mathrm{MHz}\right)$

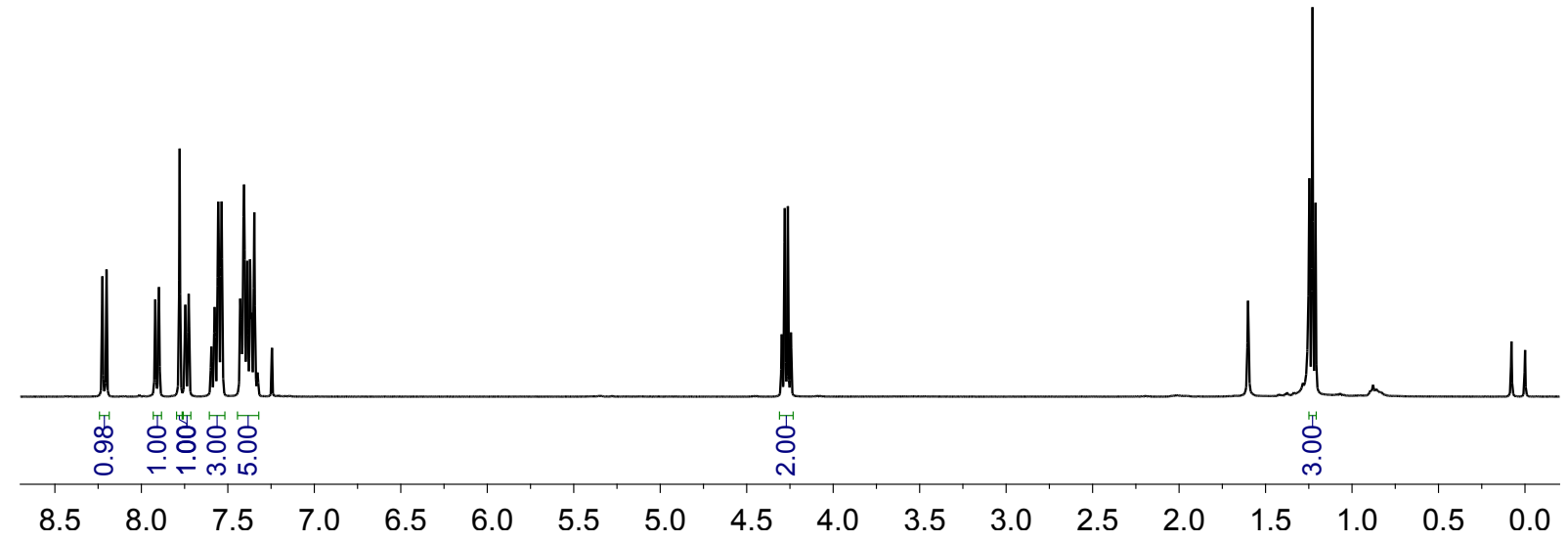

lo $\quad$ lo

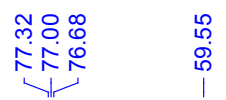

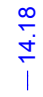

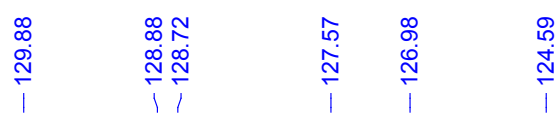
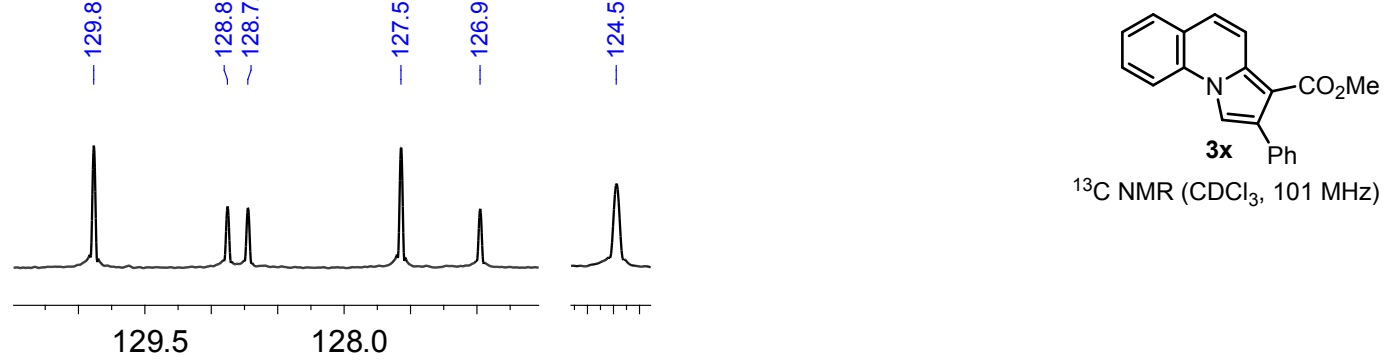

${ }^{13} \mathrm{C}$ NMR $\left(\mathrm{CDCl}_{3}, 101 \mathrm{MHz}\right)$

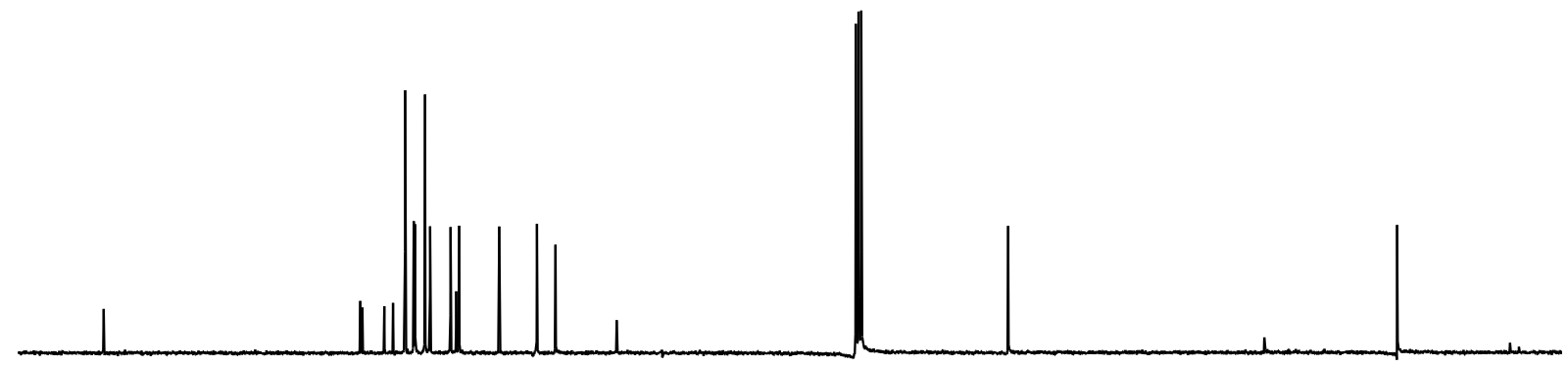

170160

$\begin{array}{llllllll}60 & 150 & 140 & 130 & 120 & 110 & 100 & 90\end{array}$

$80 \quad 70 \quad 60$

$60 \quad 50$

$\begin{array}{lllll}40 & 30 & 20 & 10 & 0\end{array}$ 

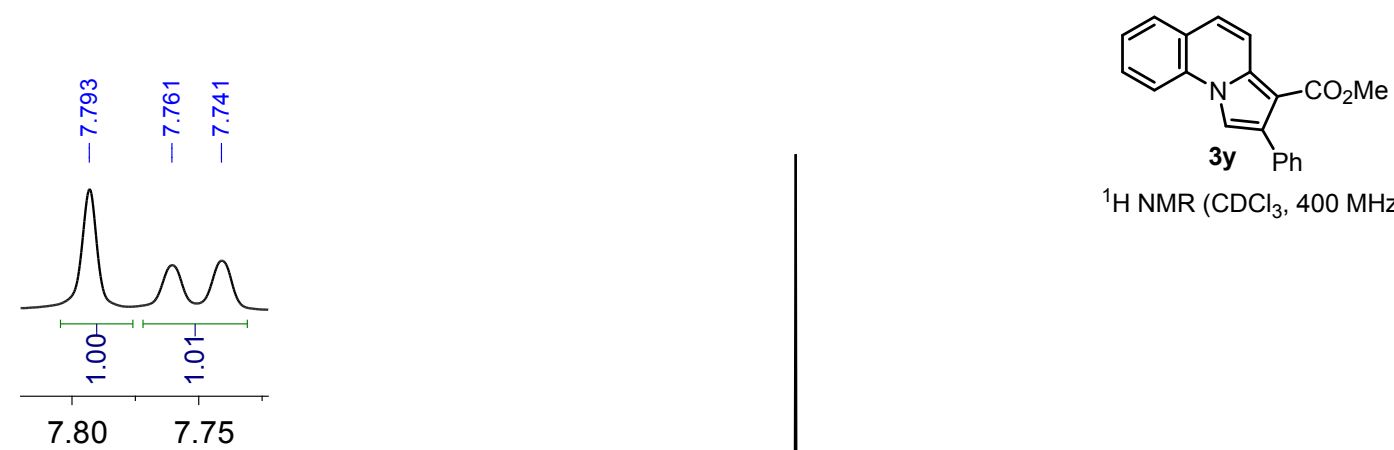

${ }^{1} \mathrm{H} \mathrm{NMR}\left(\mathrm{CDCl}_{3}, 400 \mathrm{MHz}\right)$

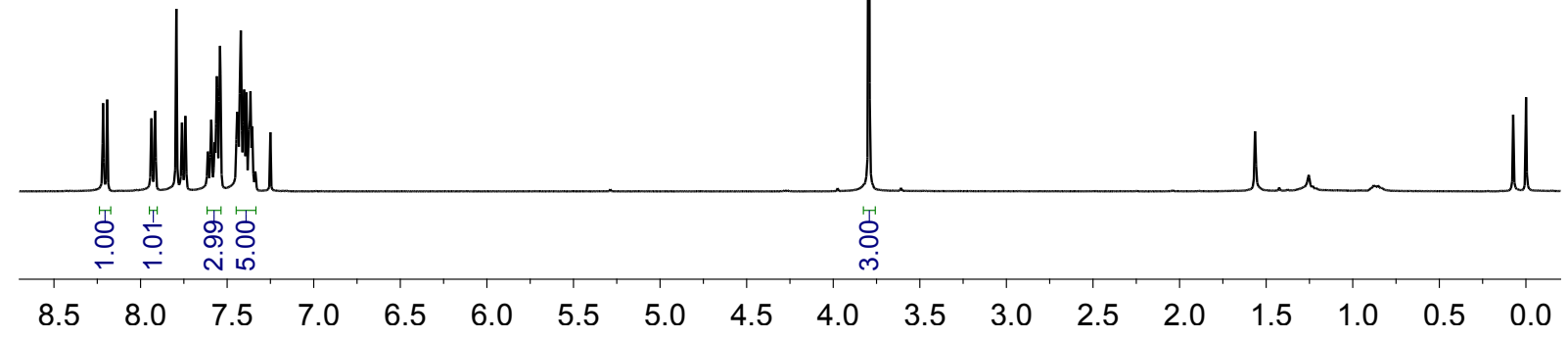

每

i⿱宀

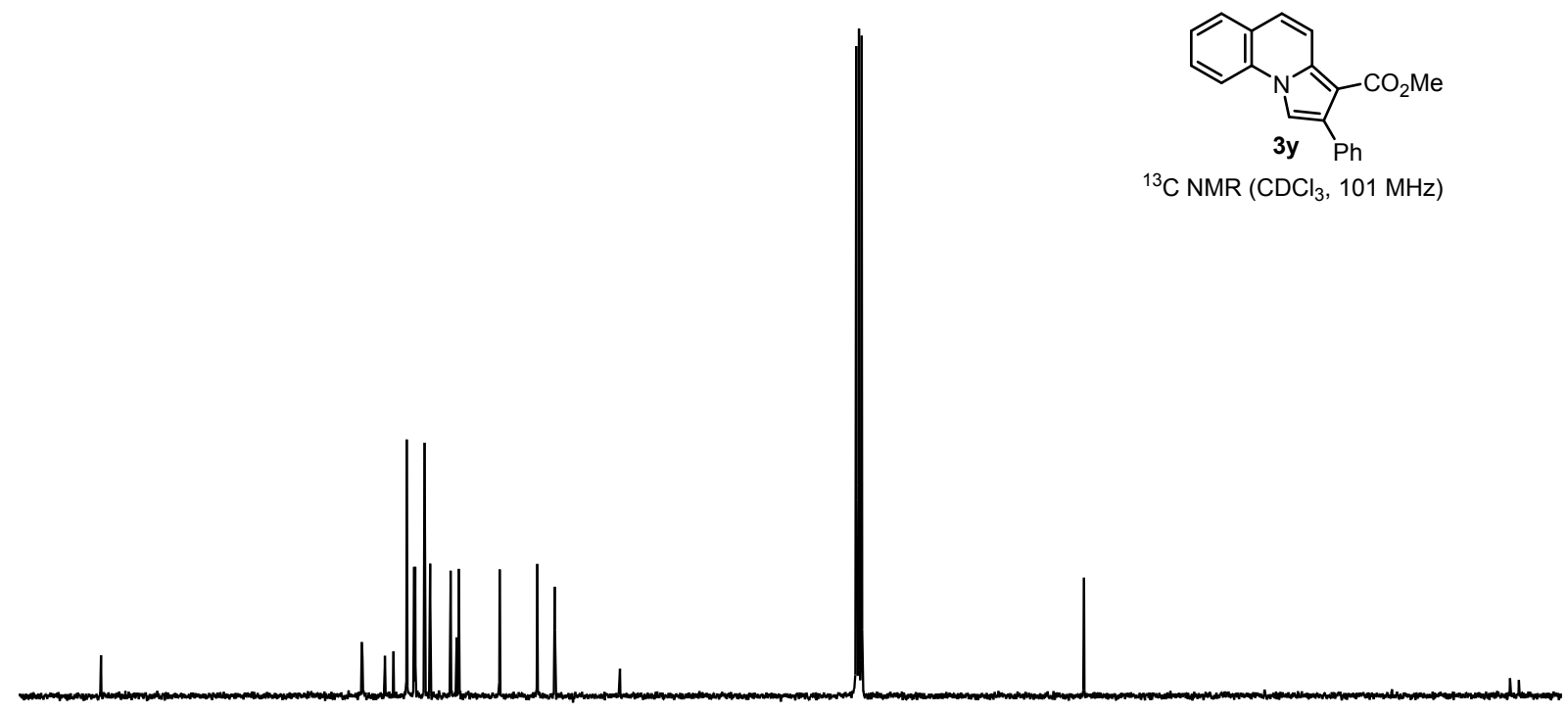

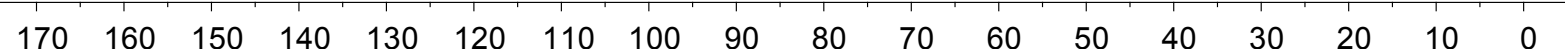




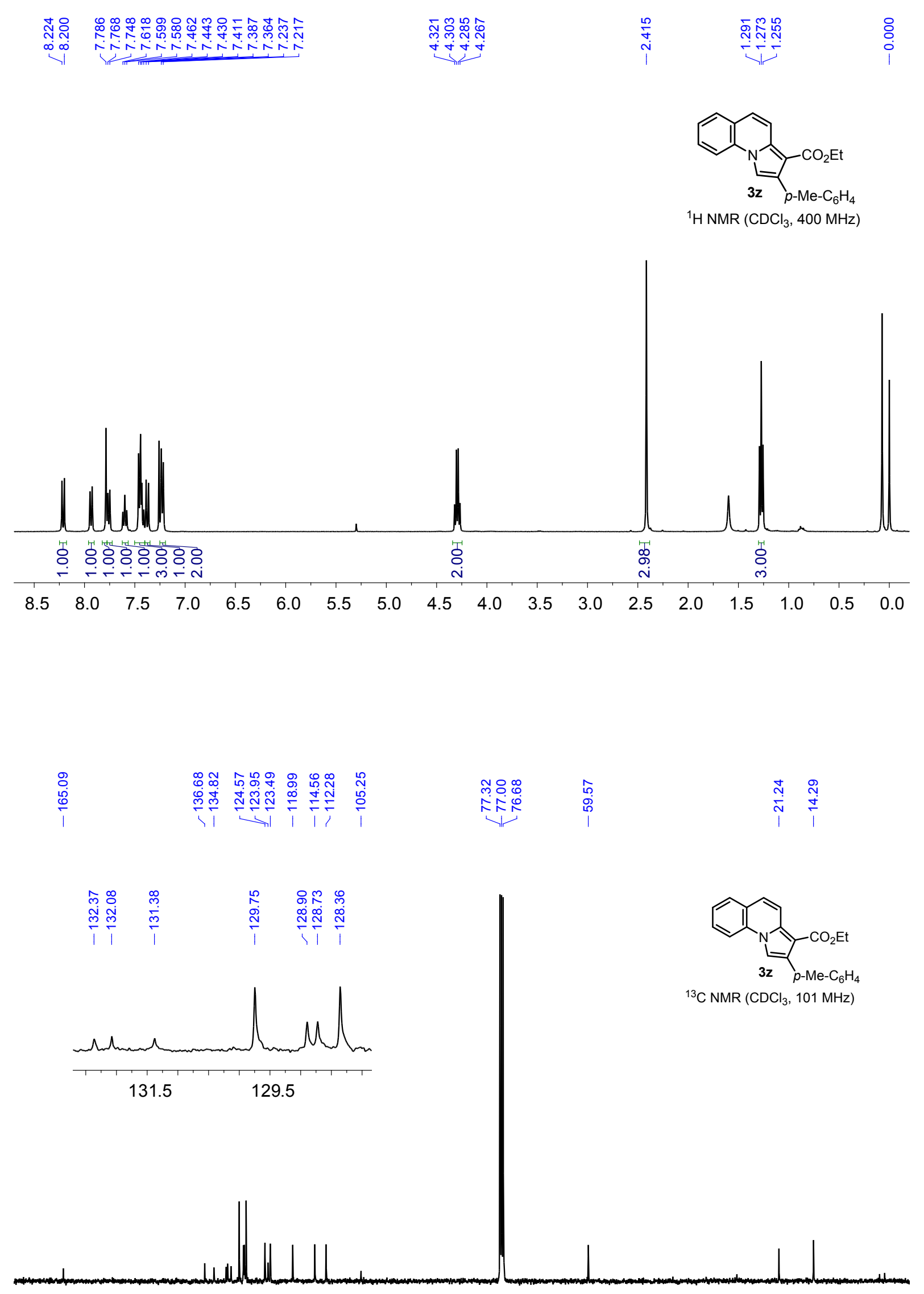

$\begin{array}{llllllllllllllllll}170 & 160 & 150 & 140 & 130 & 120 & 110 & 100 & 90 & 80 & 70 & 60 & 50 & 40 & 30 & 20 & 10 & 0\end{array}$ 

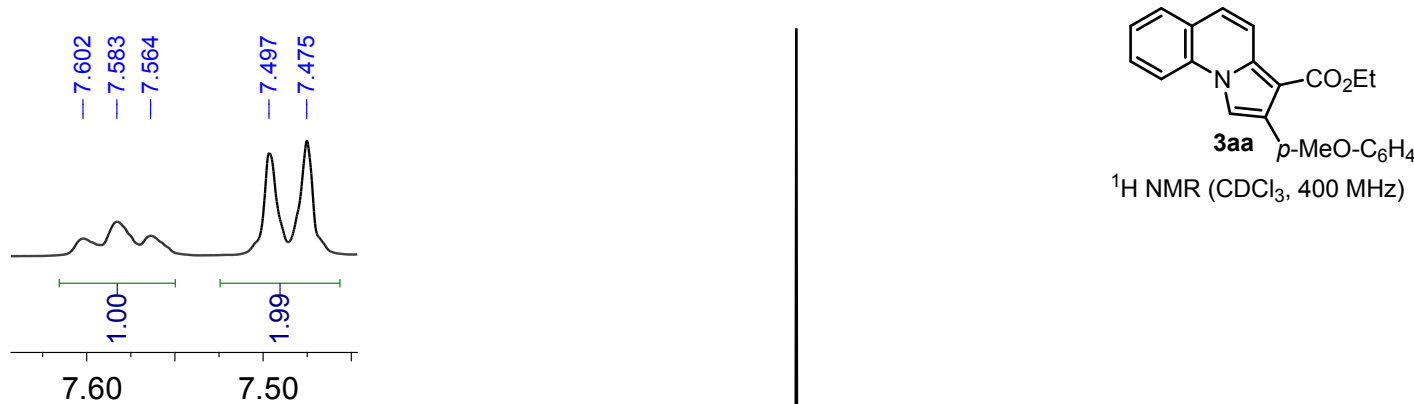

${ }^{1} \mathrm{H} \mathrm{NMR}\left(\mathrm{CDCl}_{3}, 400 \mathrm{MHz}\right)$

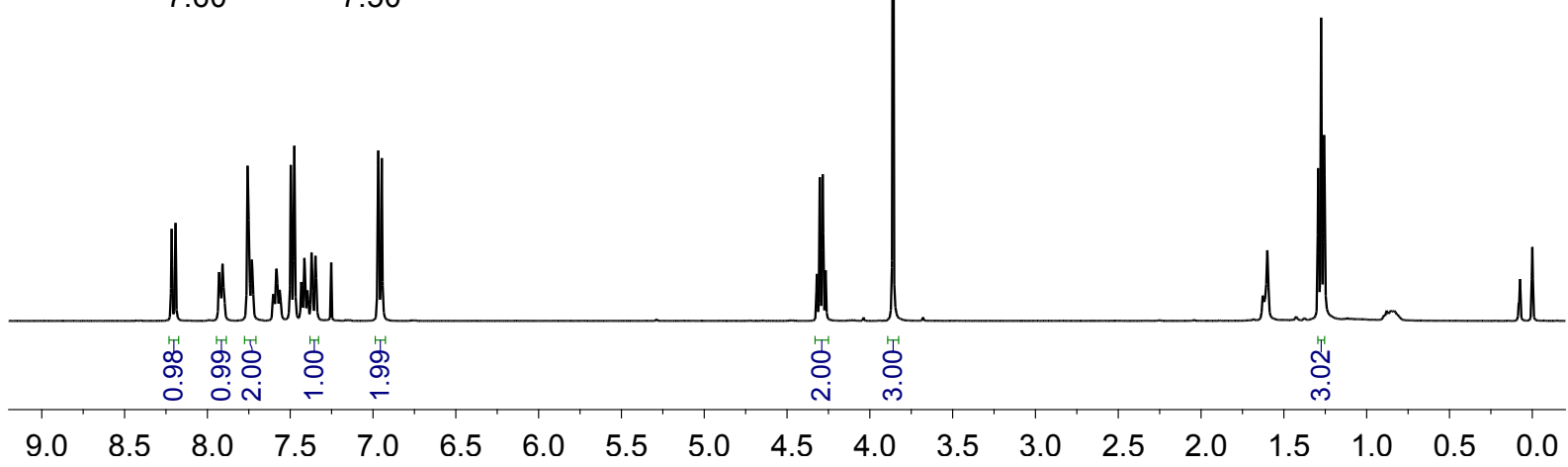

\begin{tabular}{ll}
$N$ & 0 \\
\hdashline & 0 \\
0 & 0 \\
0 & 0 \\
0 & 0 \\
1 & 1
\end{tabular}

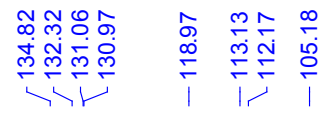

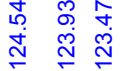

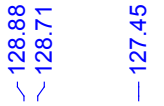

i


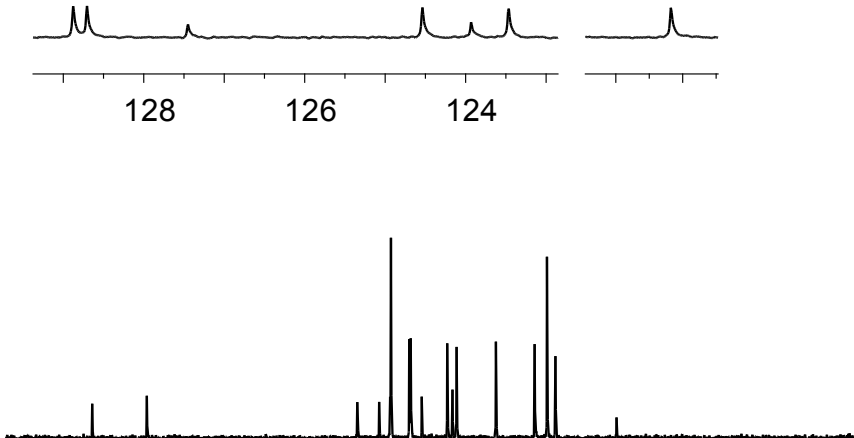

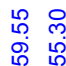

เก เก

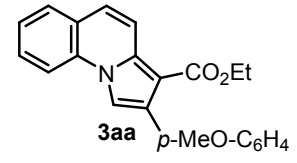

${ }^{13} \mathrm{C} \mathrm{NMR}\left(\mathrm{CDCl}_{3}, 101 \mathrm{MHz}\right)$

$\begin{array}{llllllllllllllllll}170 & 160 & 150 & 140 & 130 & 120 & 110 & 100 & 90 & 80 & 70 & 60 & 50 & 40 & 30 & 20 & 10 & 0\end{array}$ 


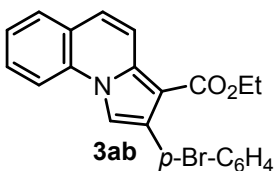

${ }^{1} \mathrm{H} \mathrm{NMR}\left(\mathrm{CDCl}_{3}, 400 \mathrm{MHz}\right)$

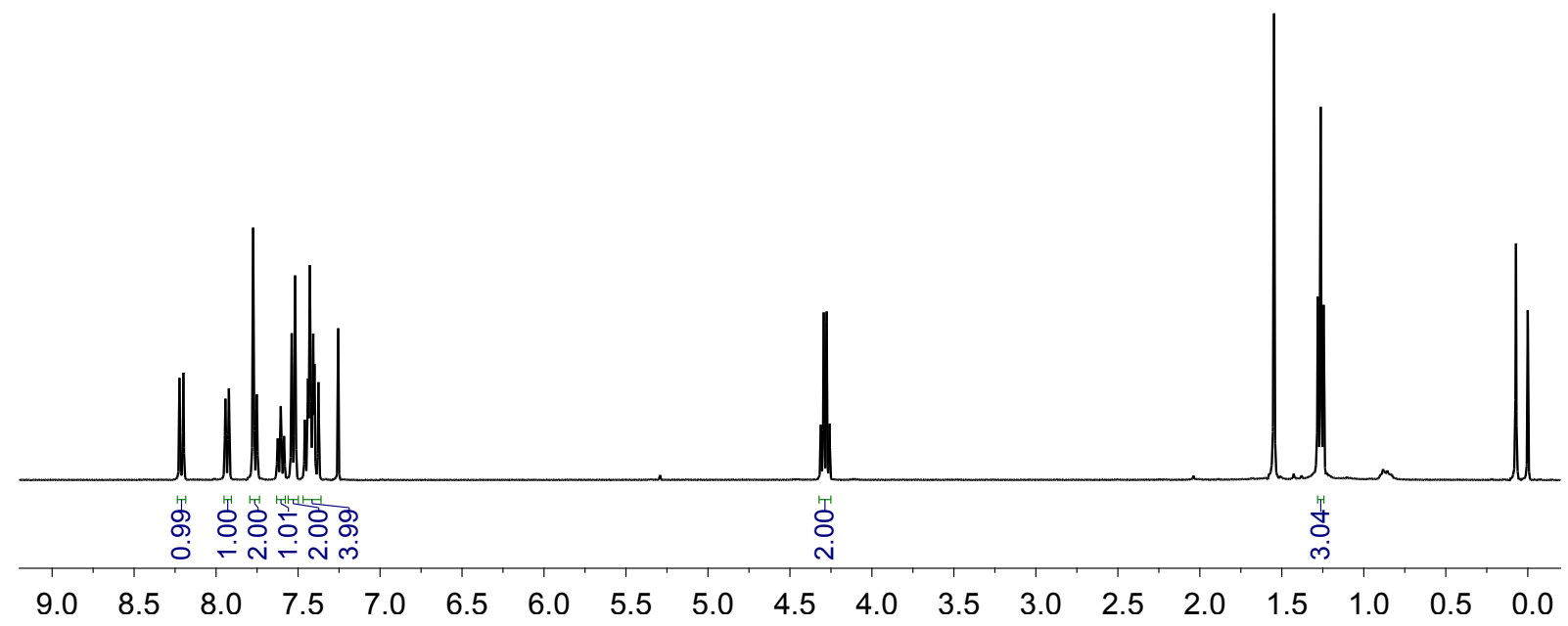

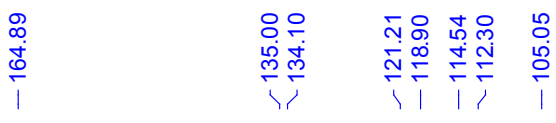

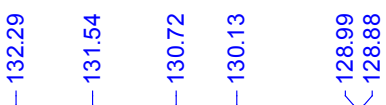

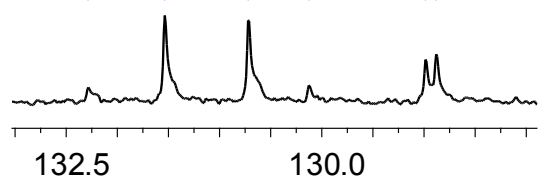

R.

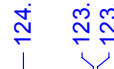
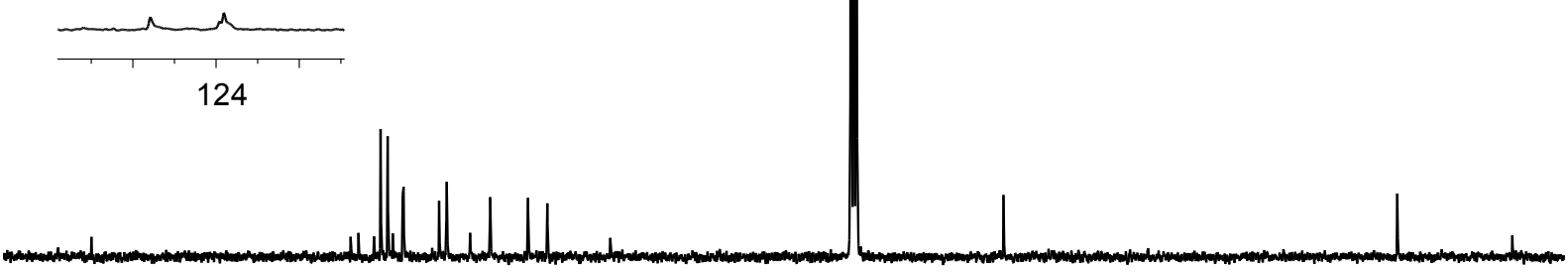

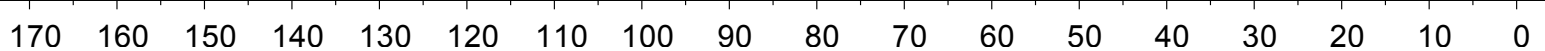




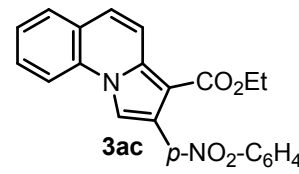

${ }^{1} \mathrm{H} \mathrm{NMR}\left(\mathrm{CDCl}_{3}, 400 \mathrm{MHz}\right)$

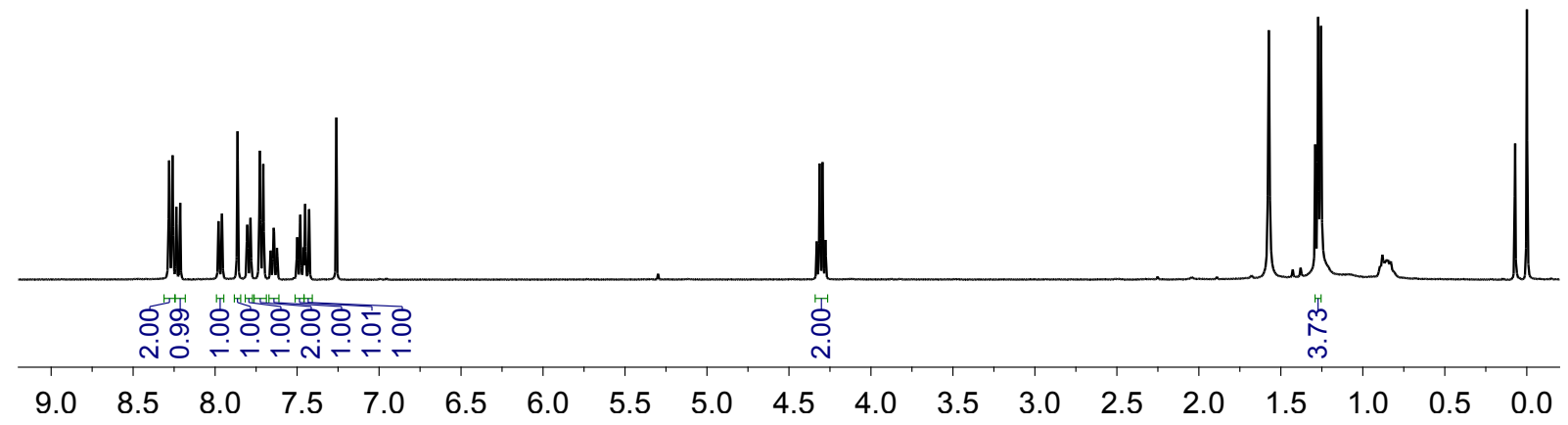

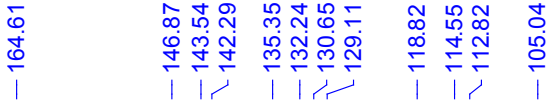

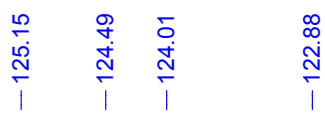

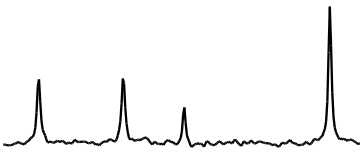

124.0

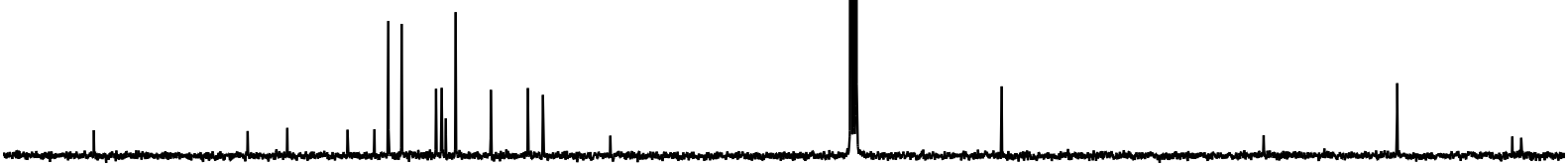

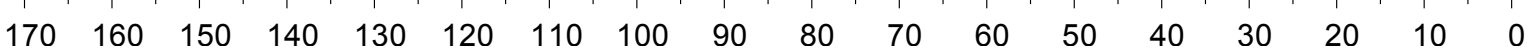




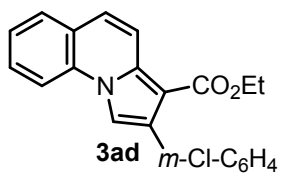

${ }^{1} \mathrm{H} \mathrm{NMR}\left(\mathrm{CDCl}_{3}, 400 \mathrm{MHz}\right)$
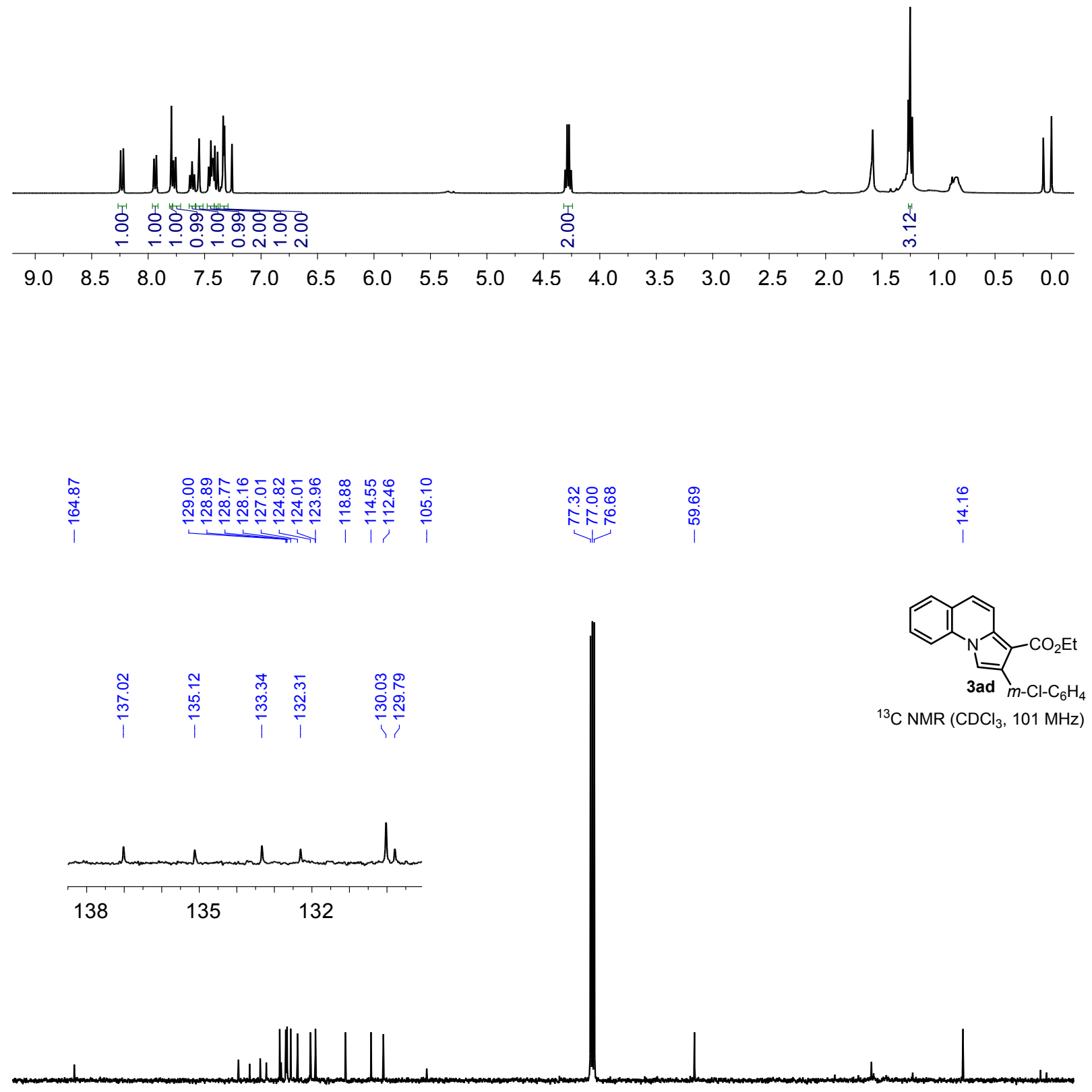

$\begin{array}{llllllllllllllllll}170 & 160 & 150 & 140 & 130 & 120 & 110 & 100 & 90 & 80 & 70 & 60 & 50 & 40 & 30 & 20 & 10 & 0\end{array}$ 


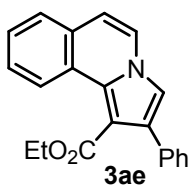

${ }^{1} \mathrm{H} \mathrm{NMR}\left(\mathrm{CDCl}_{3}, 400 \mathrm{MHz}\right)$
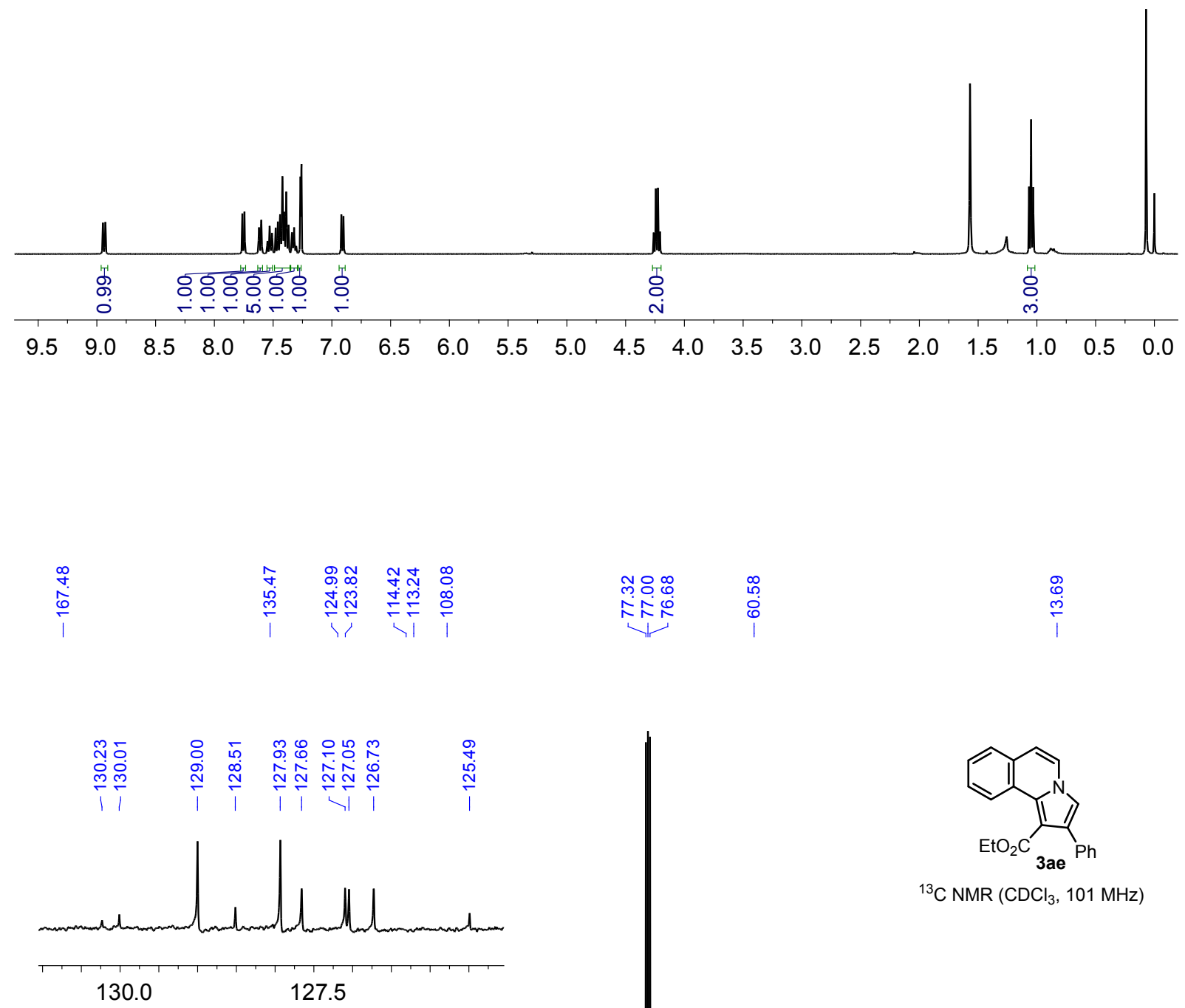

${ }^{13} \mathrm{C}$ NMR $\left(\mathrm{CDCl}_{3}, 101 \mathrm{MHz}\right)$

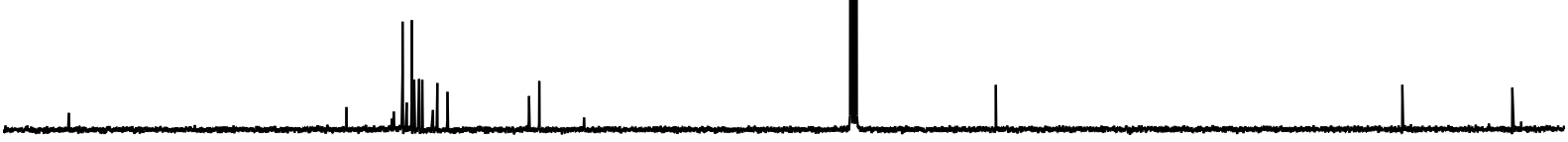

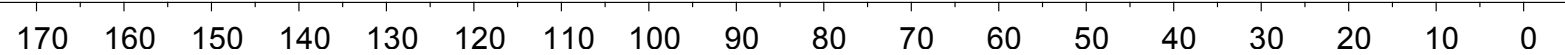


i⿱宀⿻心㇒日
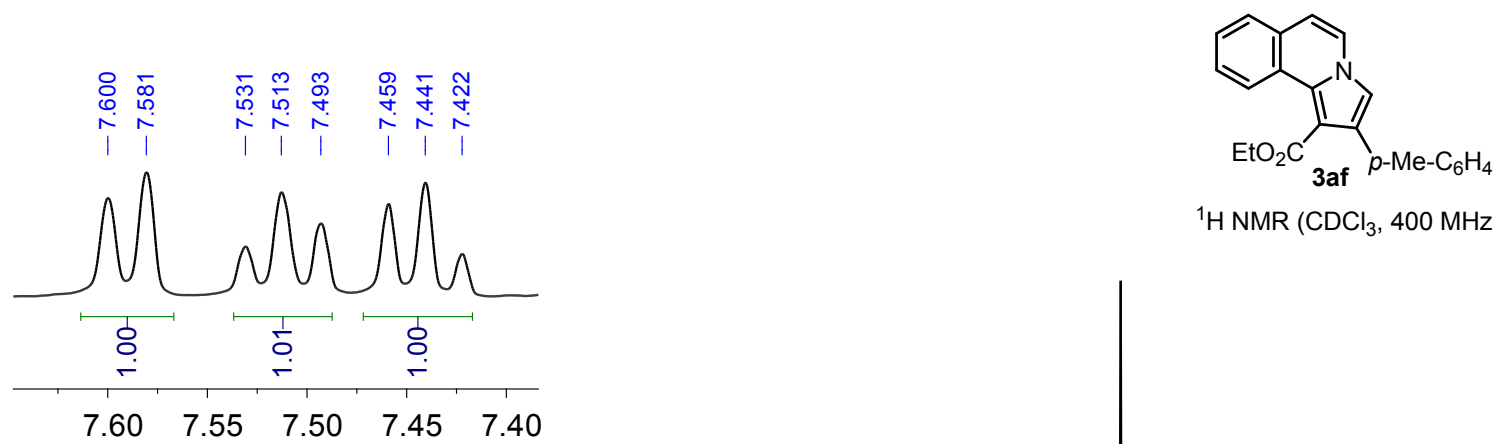

${ }^{1} \mathrm{H} \mathrm{NMR}\left(\mathrm{CDCl}_{3}, 400 \mathrm{MHz}\right)$
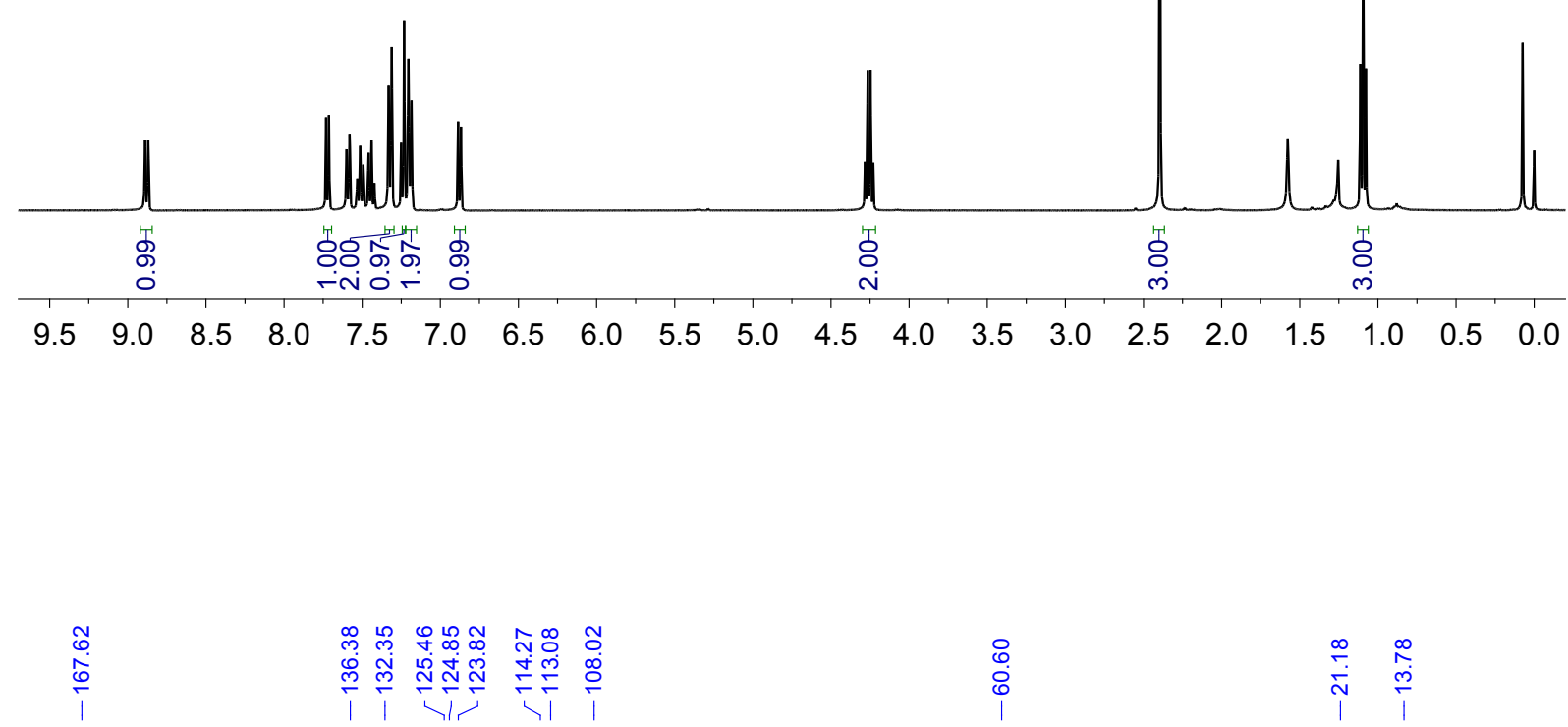

0
8
0
1

$\stackrel{\infty}{\check{\sim}} \stackrel{\infty}{\stackrel{m}{\sim}}$

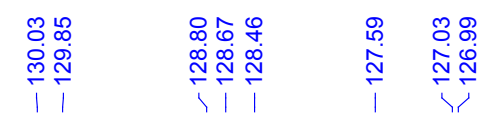
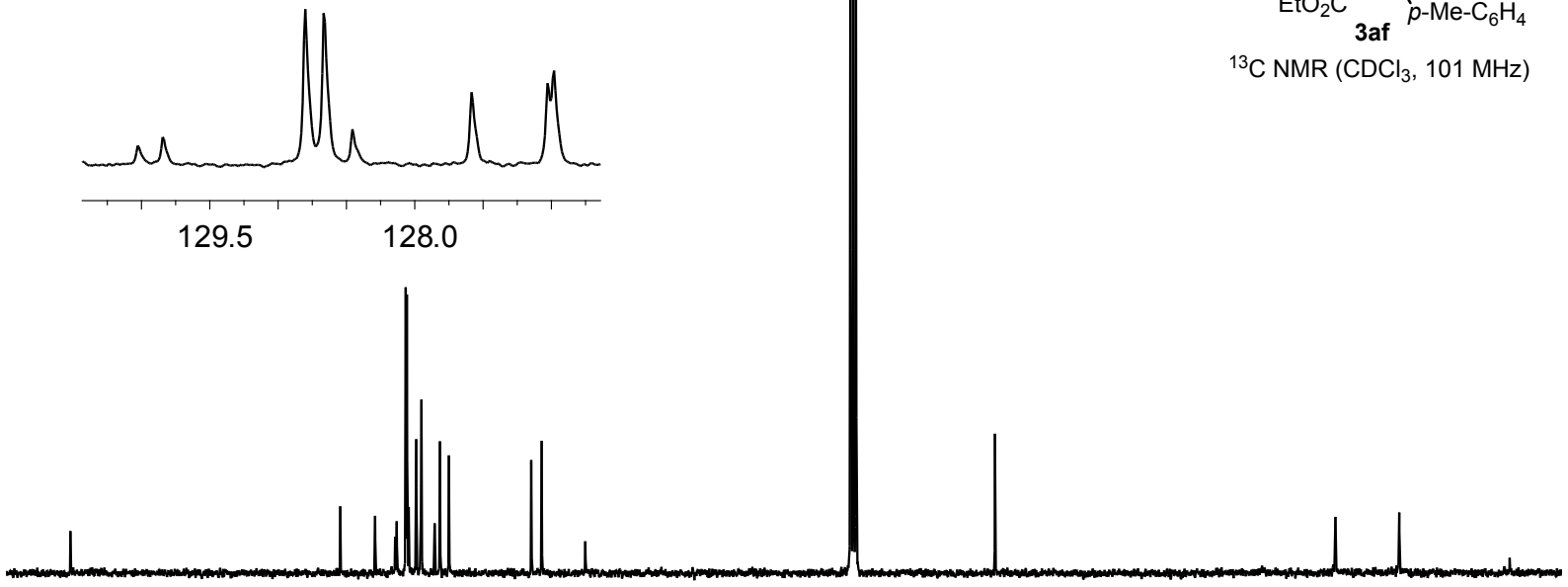

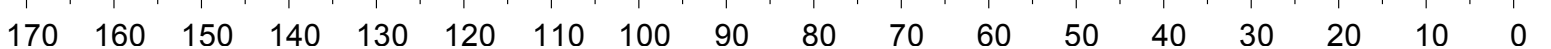




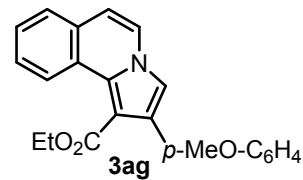

${ }^{1} \mathrm{H} \mathrm{NMR}\left(\mathrm{CDCl}_{3}, 400 \mathrm{MHz}\right)$

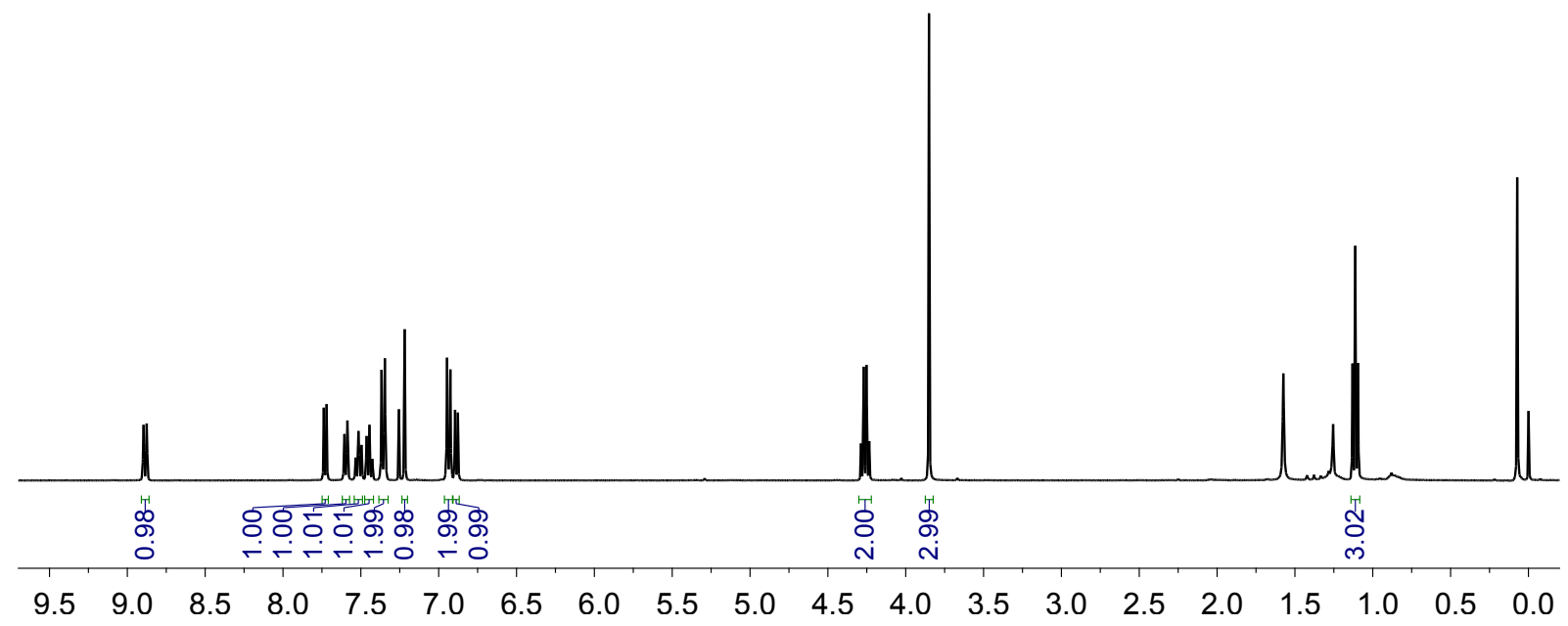

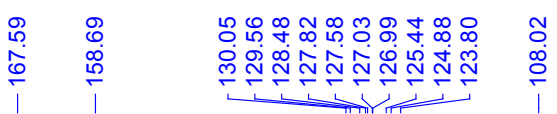

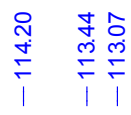

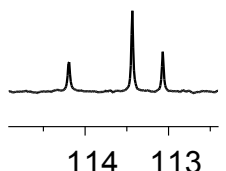

草

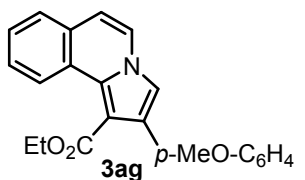

$\left.{ }^{13} \mathrm{C} \mathrm{NMR} \mathrm{(CDCl}, 101 \mathrm{MHz}\right)$

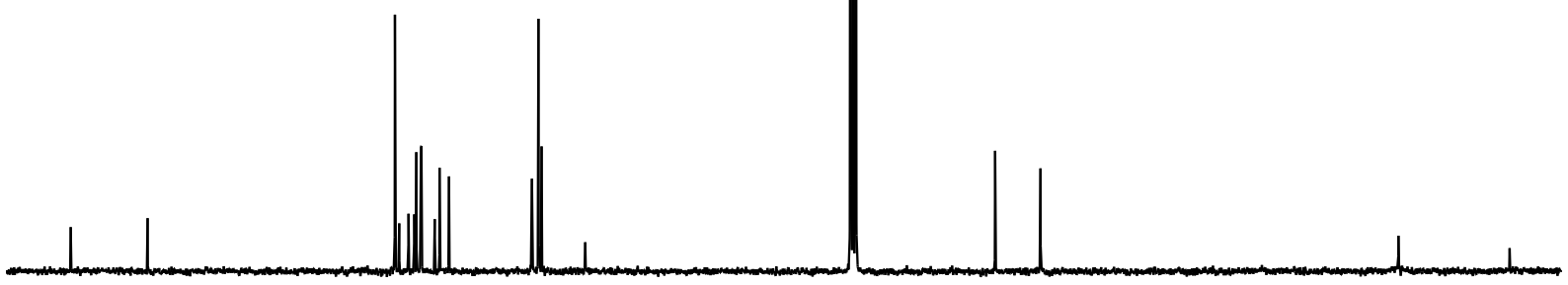

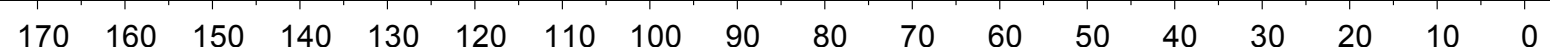




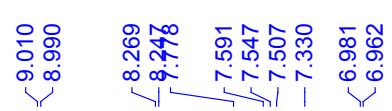

员牙

NiNiN

म1 17?



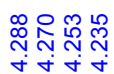

更过

望

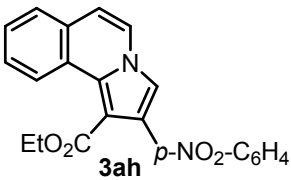

${ }^{1} \mathrm{H} \mathrm{NMR}\left(\mathrm{CDCl}_{3}, 400 \mathrm{MHz}\right)$

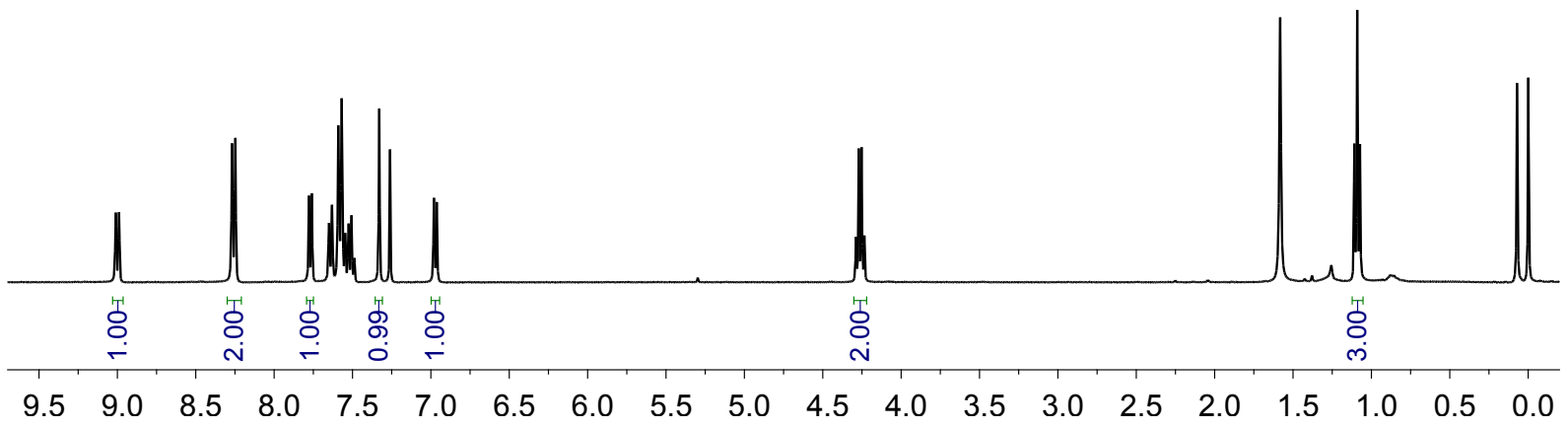

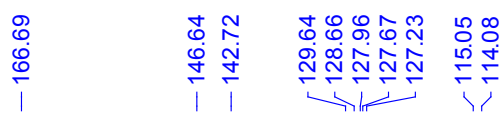

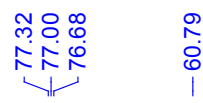

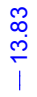

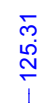

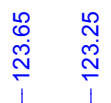

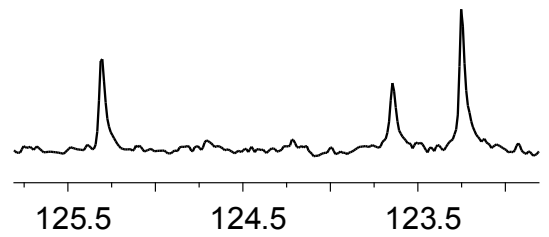

${ }^{13} \mathrm{C} \mathrm{NMR}\left(\mathrm{CDCl}_{3}, 101 \mathrm{MHz}\right)$
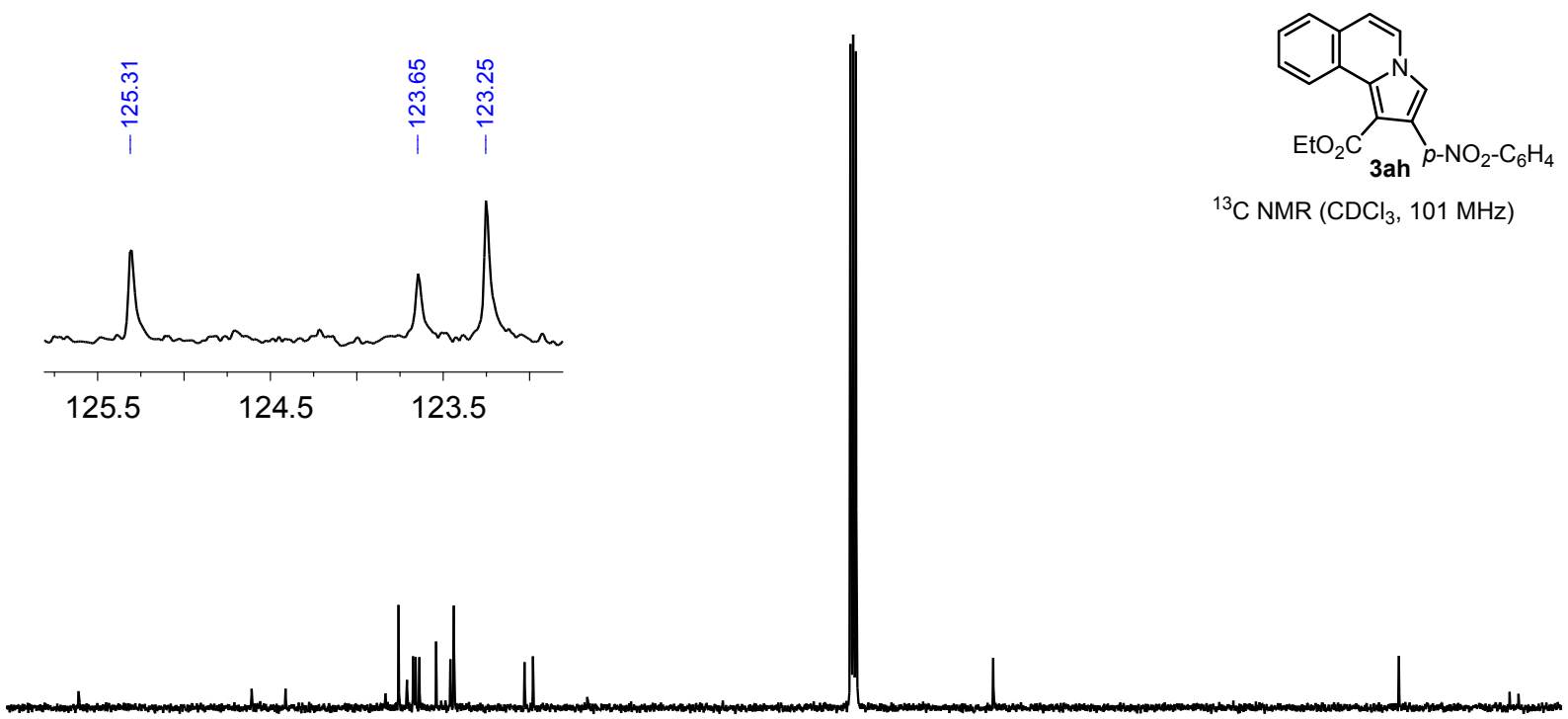

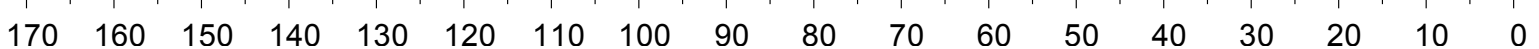




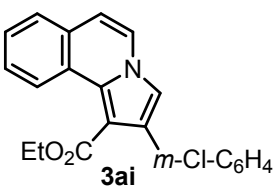

${ }^{1} \mathrm{H} \mathrm{NMR}\left(\mathrm{CDCl}_{3}, 400 \mathrm{MHz}\right)$
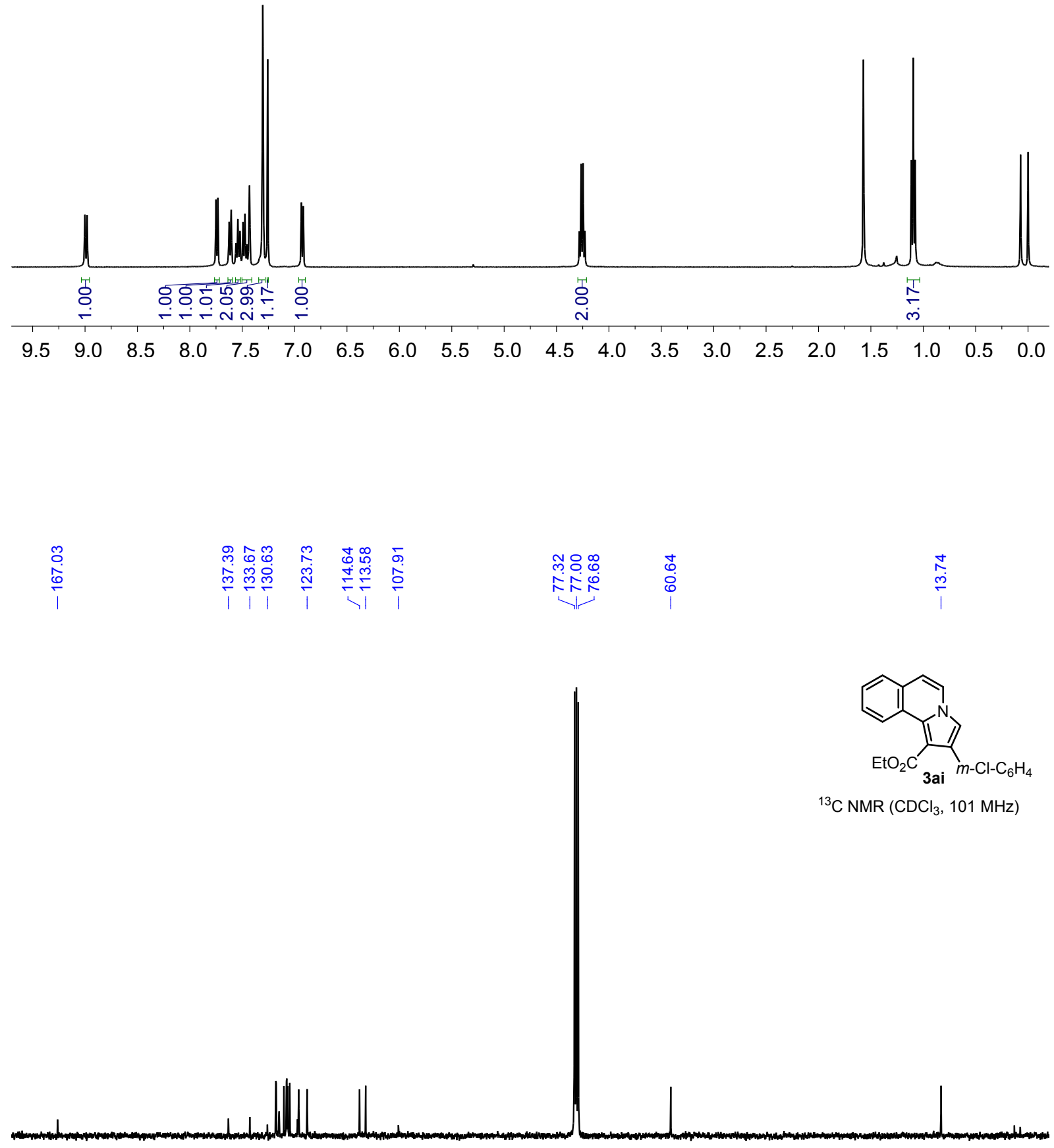

$\begin{array}{llllllllllllllllll}170 & 160 & 150 & 140 & 130 & 120 & 110 & 100 & 90 & 80 & 70 & 60 & 50 & 40 & 30 & 20 & 10 & 0\end{array}$ 


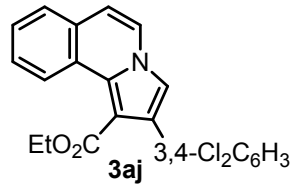

${ }^{1} \mathrm{H}$ NMR $\left(\mathrm{CDCl}_{3}, 400 \mathrm{MHz}\right)$

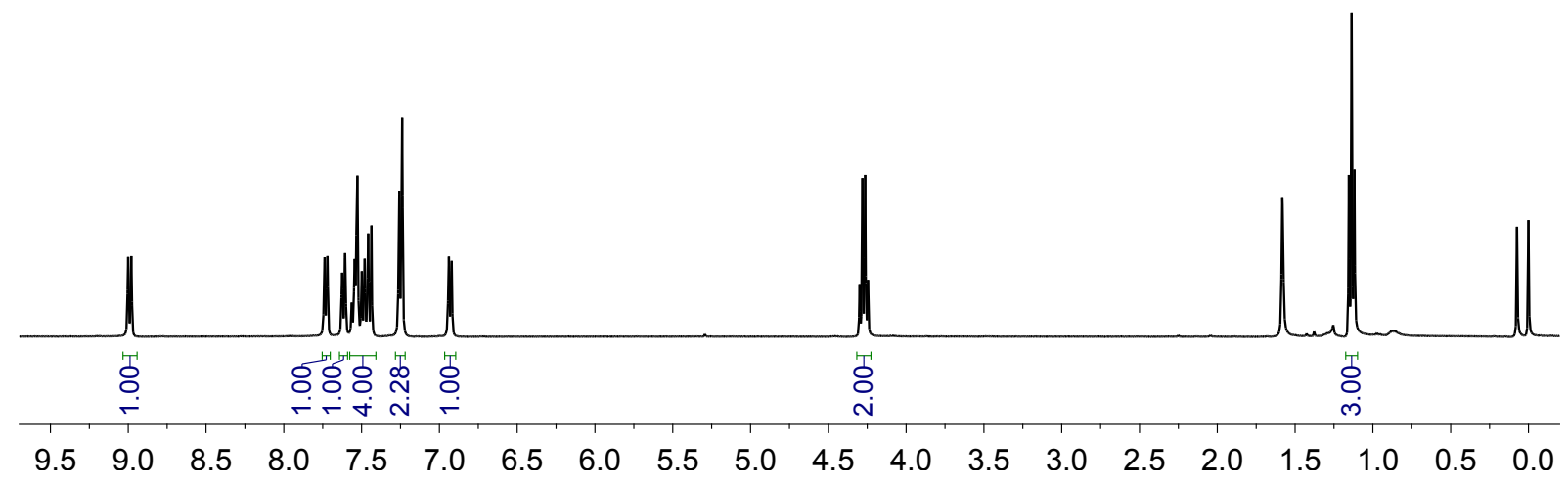

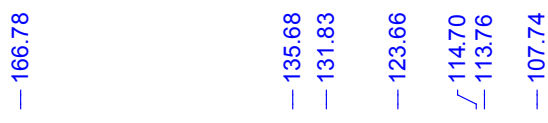

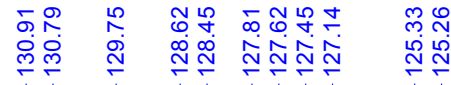
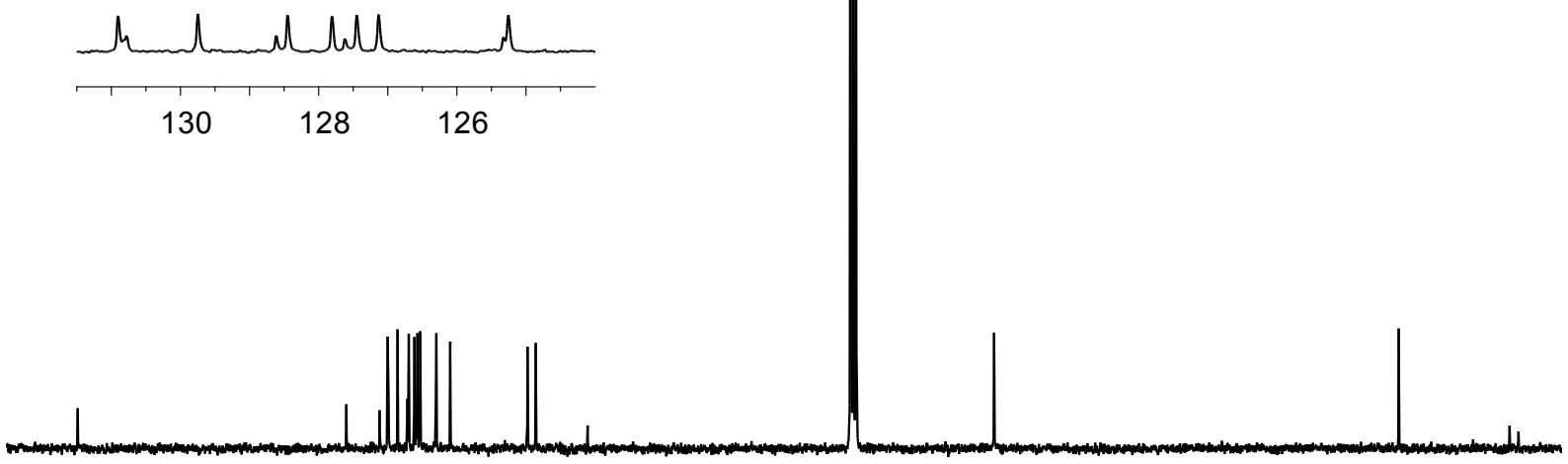

170

$\begin{array}{llll}130 & 120 & 110 & 100\end{array}$

8
0
8
1

$\stackrel{\substack{\infty \\ \infty}}{\stackrel{m}{\sim}}$

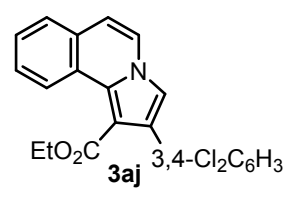

${ }^{13} \mathrm{C} \mathrm{NMR}\left(\mathrm{CDCl}_{3}, 101 \mathrm{MHz}\right)$ 


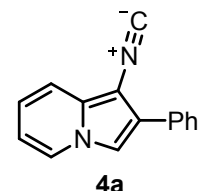

${ }^{1} \mathrm{H} \mathrm{NMR}\left(\mathrm{CDCl}_{3}, 400 \mathrm{MHz}\right)$
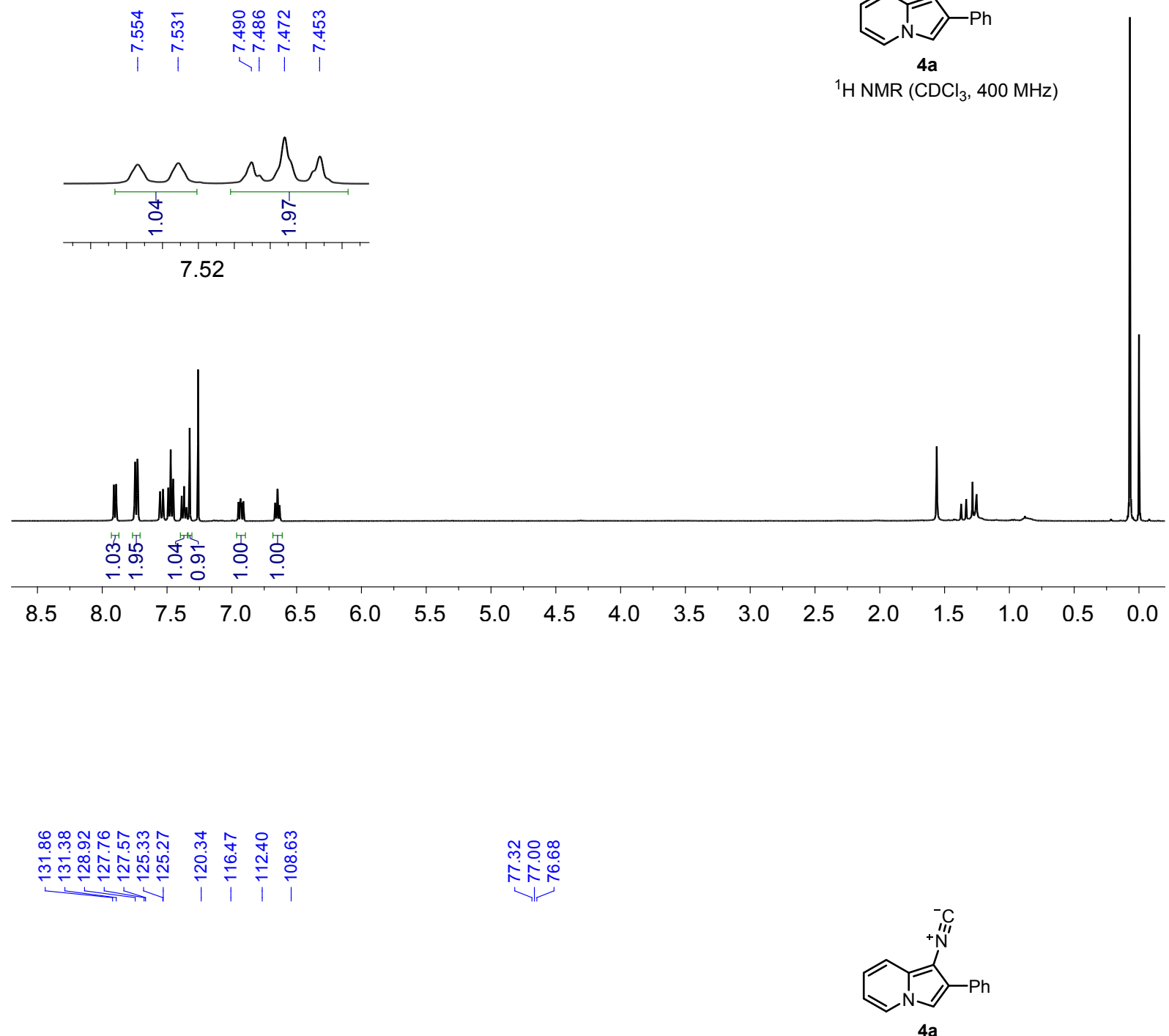

${ }^{13} \mathrm{C} \mathrm{NMR}\left(\mathrm{CDCl}_{3}, 101 \mathrm{MHz}\right)$

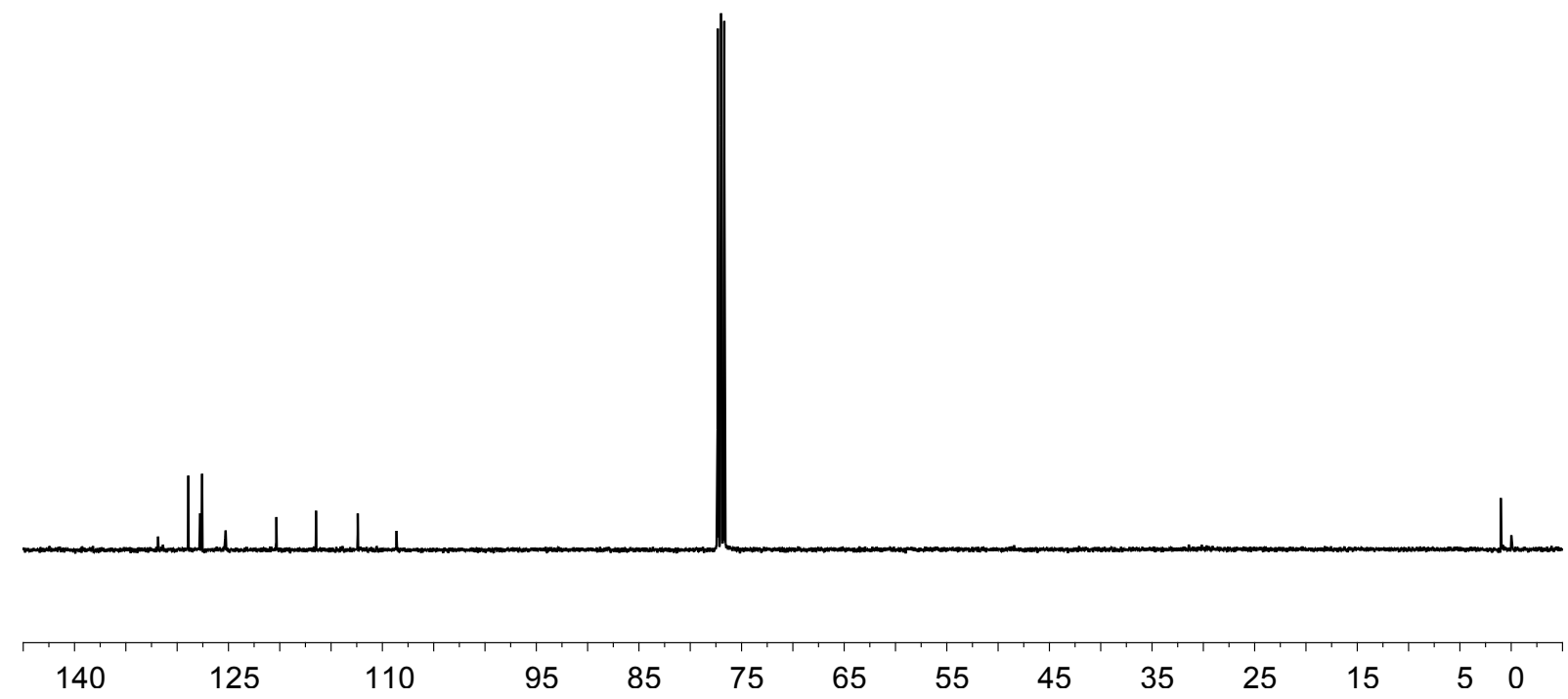




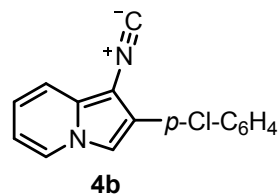

${ }^{1} \mathrm{H} \mathrm{NMR}\left(\mathrm{CDCl}_{3}, 400 \mathrm{MHz}\right)$
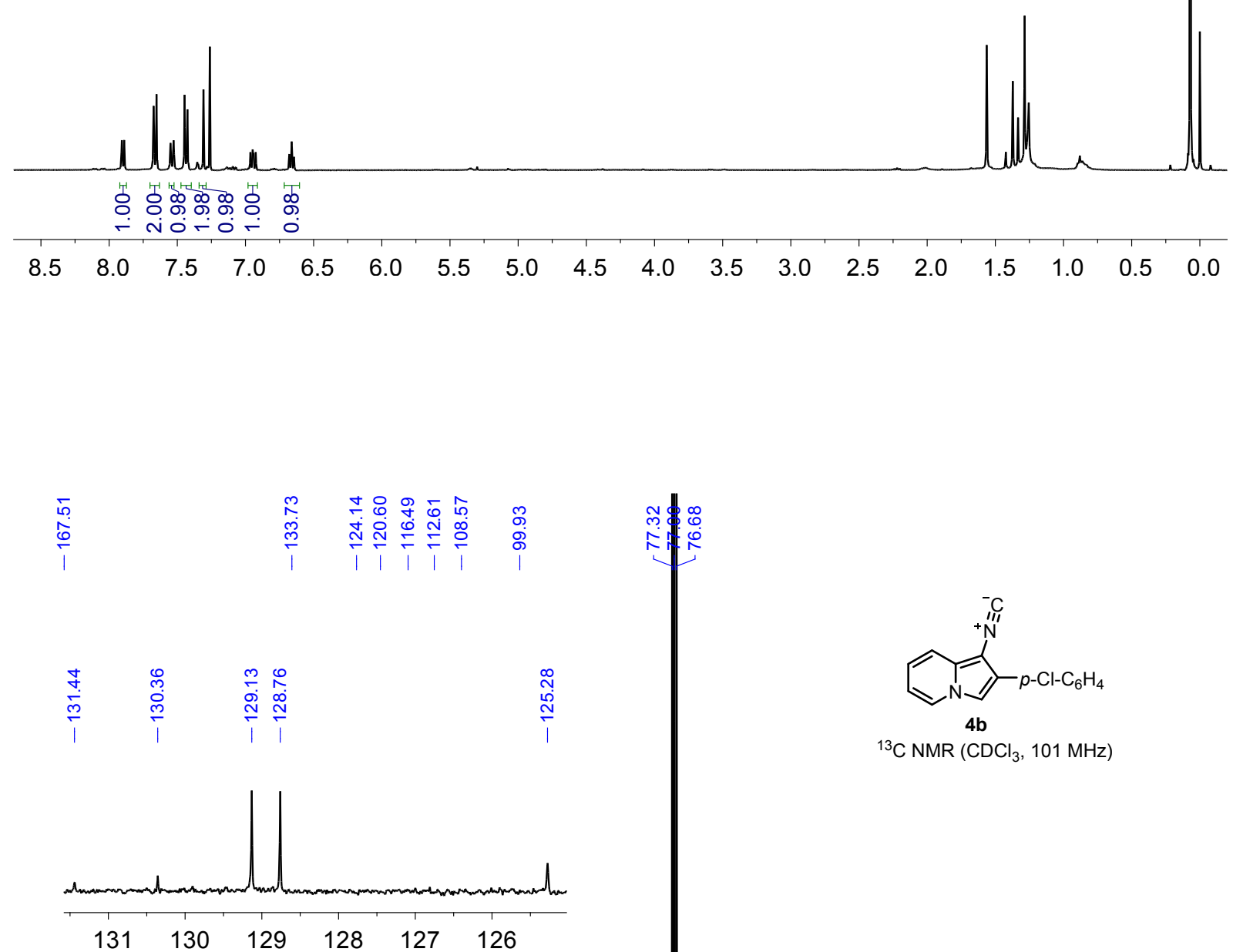

${ }^{13} \mathrm{C}$ NMR $\left(\mathrm{CDCl}_{3}, 101 \mathrm{MHz}\right)$

$\begin{array}{llllllllllllllllll}170 & 160 & 150 & 140 & 130 & 120 & 110 & 100 & 90 & 80 & 70 & 60 & 50 & 40 & 30 & 20 & 10 & 0\end{array}$



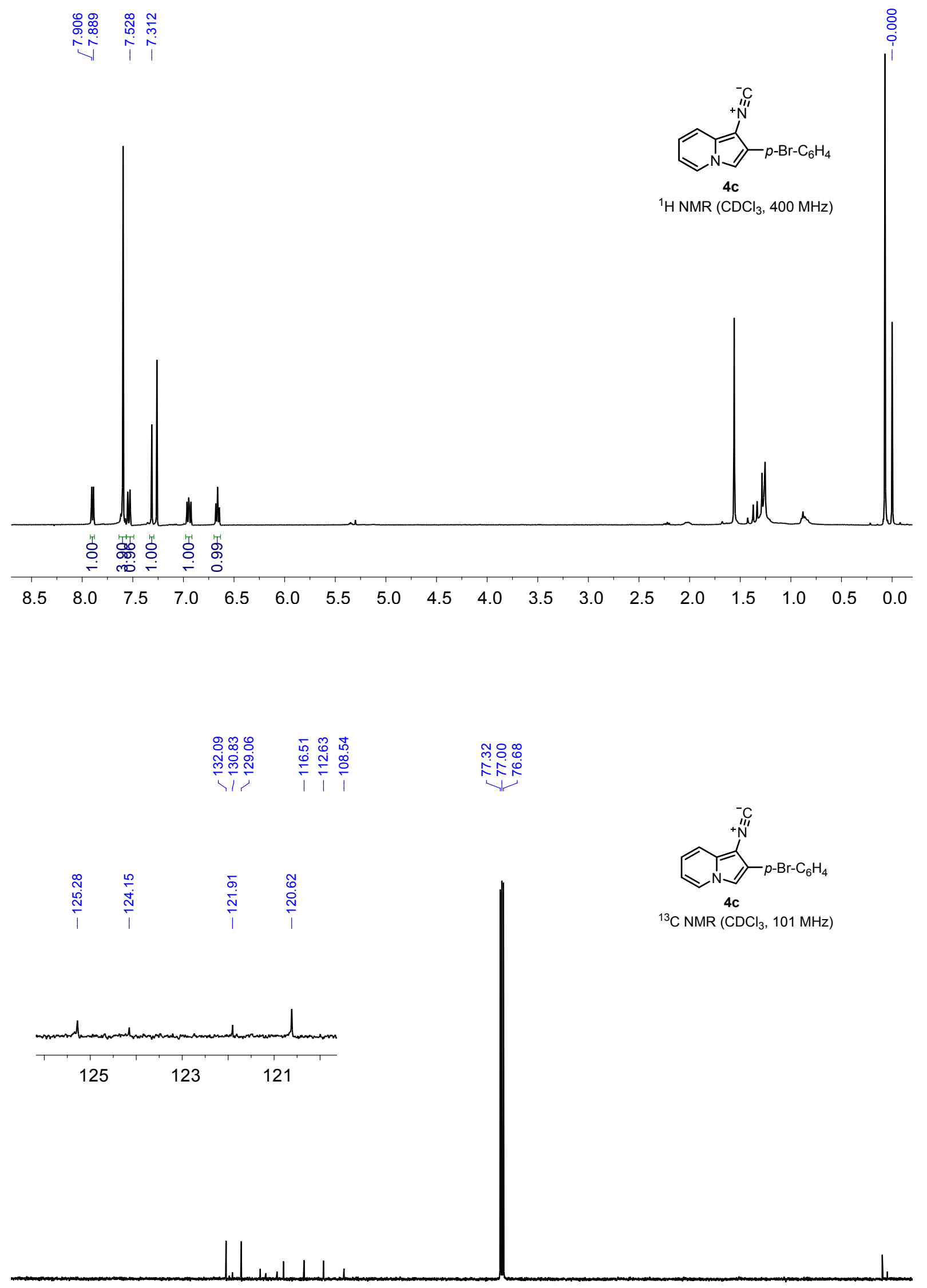

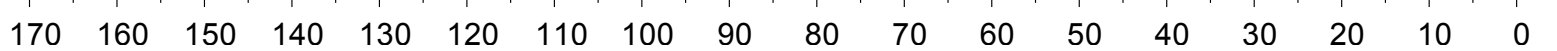




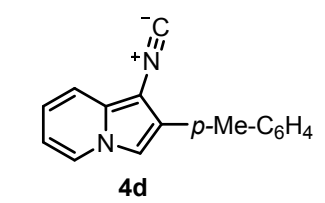

${ }^{1} \mathrm{H} \mathrm{NMR}\left(\mathrm{CDCl}_{3}, 400 \mathrm{MHz}\right)$

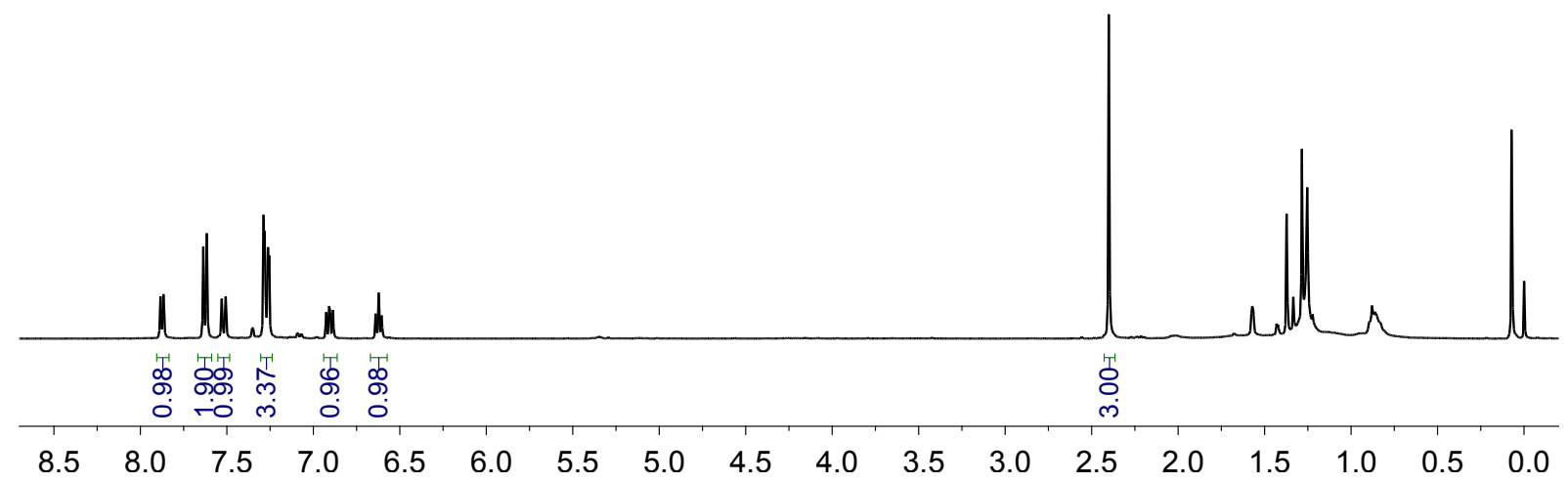

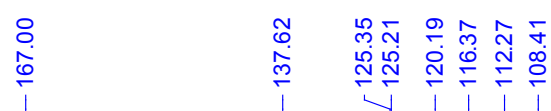

$$
\stackrel{\bar{m}}{\stackrel{m}{5}}
$$

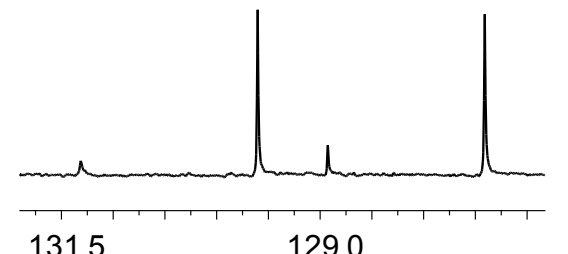

131.5 129.0

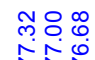

사요

$\stackrel{\stackrel{n}{N}}{i}$

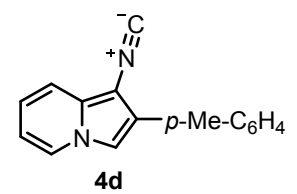

${ }^{13} \mathrm{C}$ NMR $\left(\mathrm{CDCl}_{3}, 101 \mathrm{MHz}\right)$

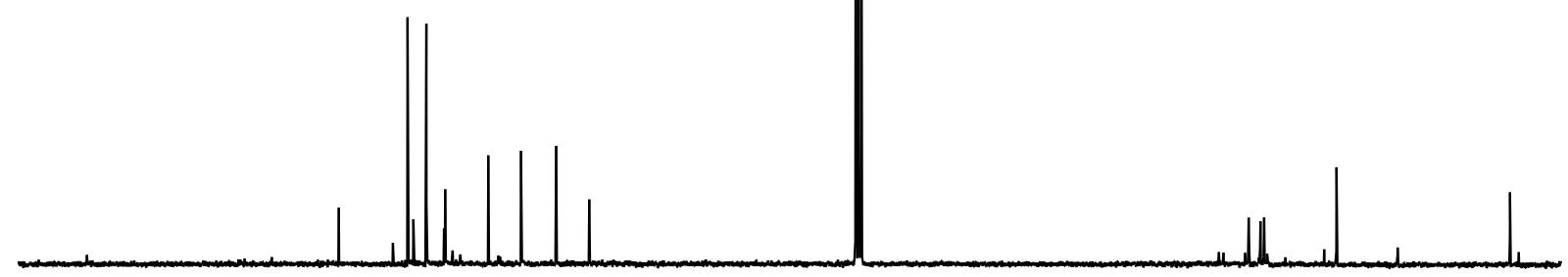

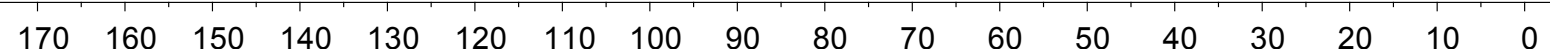




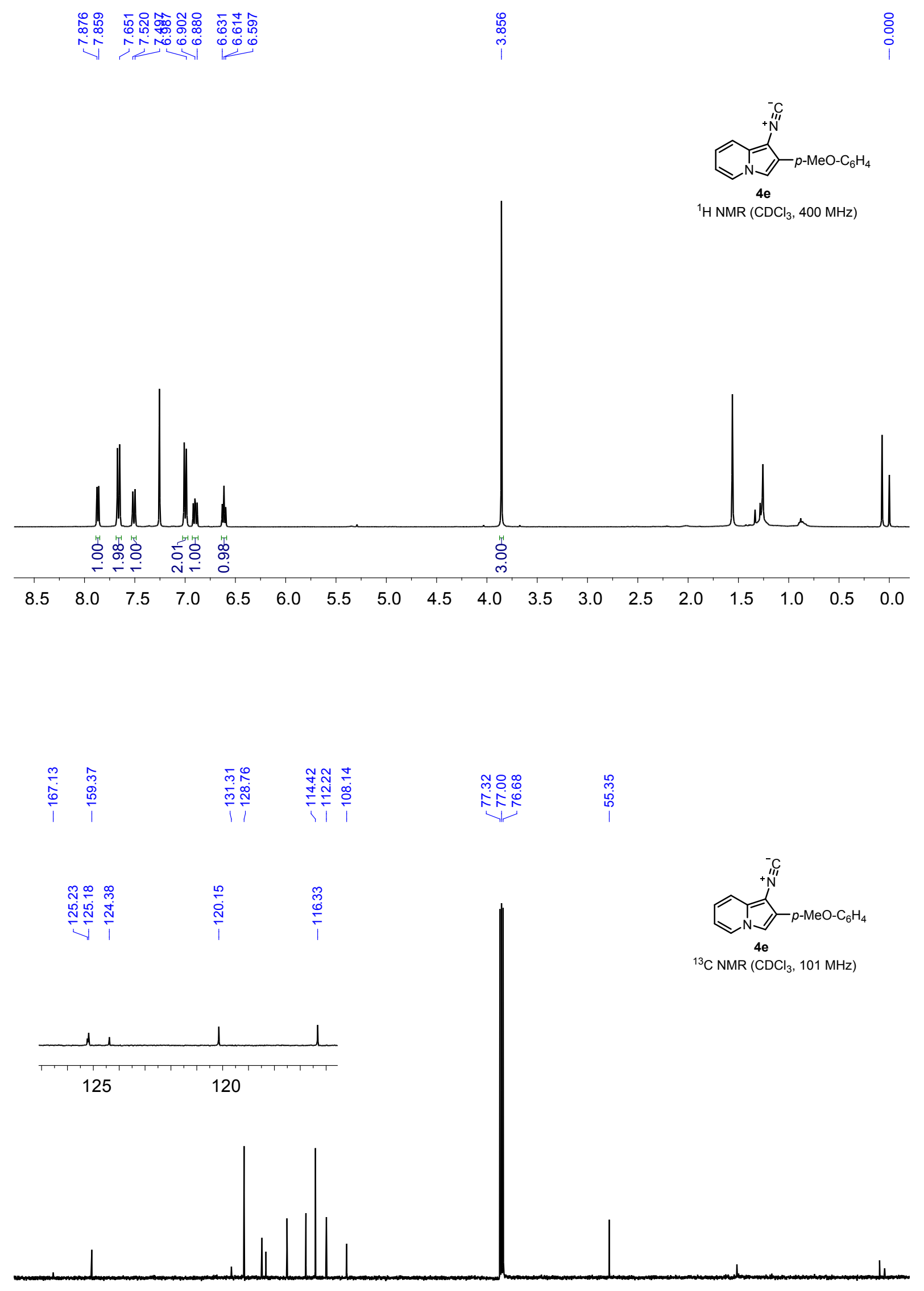

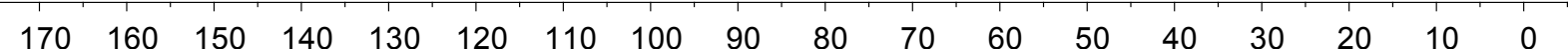




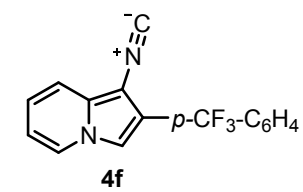

${ }^{1} \mathrm{H} \mathrm{NMR}\left(\mathrm{CDCl}_{3}, 400 \mathrm{MHz}\right)$
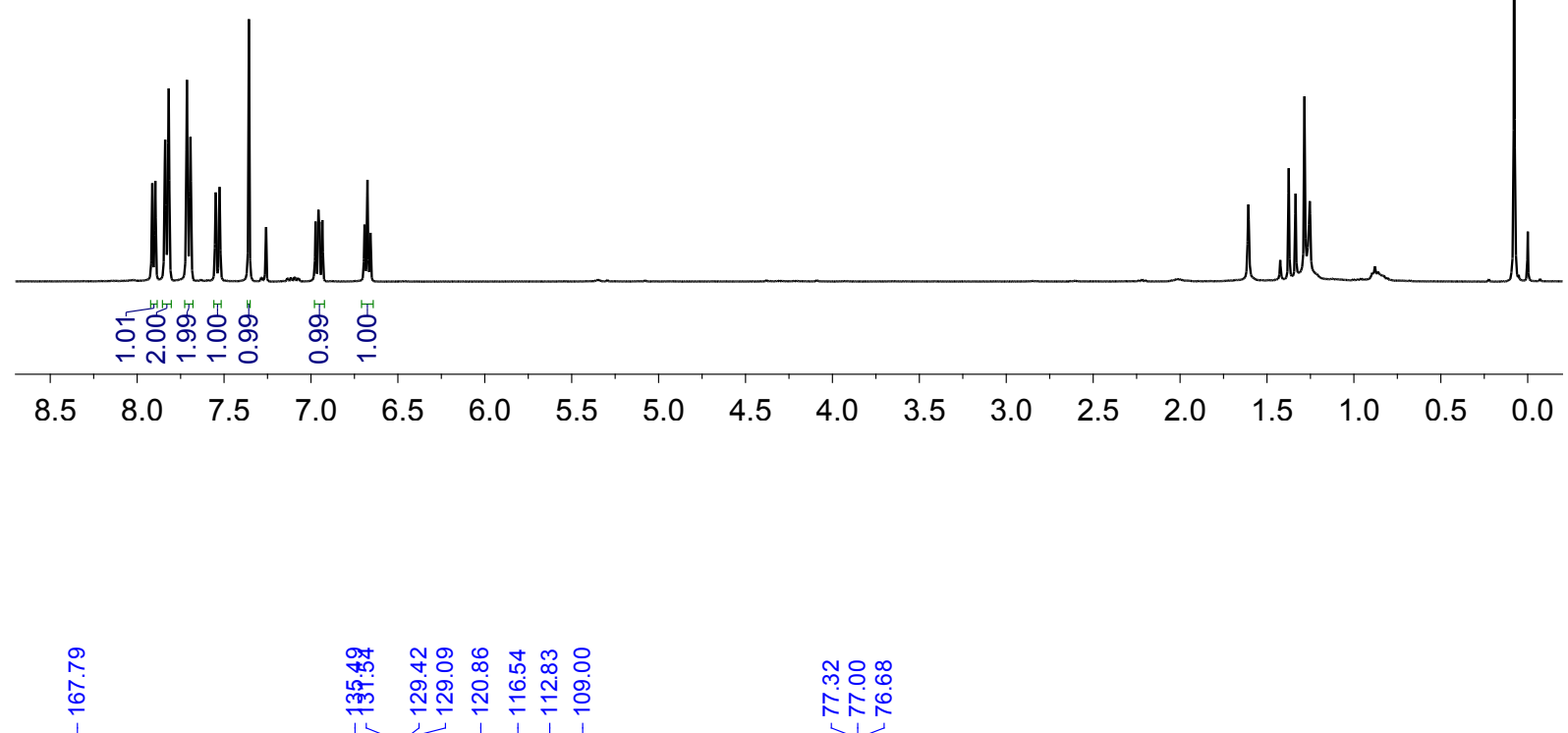

I
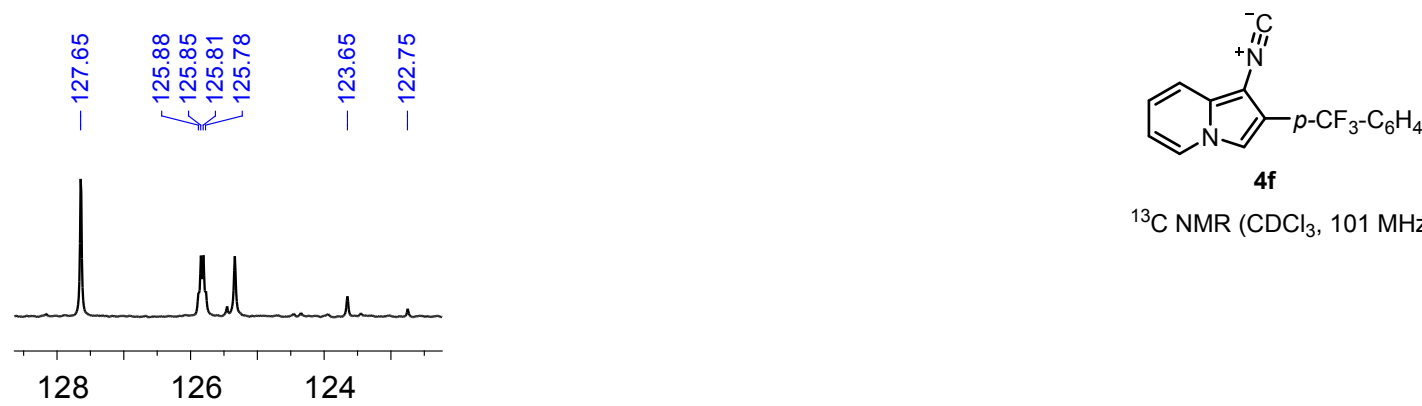

${ }^{13} \mathrm{C} \mathrm{NMR}\left(\mathrm{CDCl}_{3}, 101 \mathrm{MHz}\right)$

$\stackrel{\circ}{\stackrel{+}{*}} \stackrel{\stackrel{m}{\sim}}{\stackrel{1}{\sim}}$

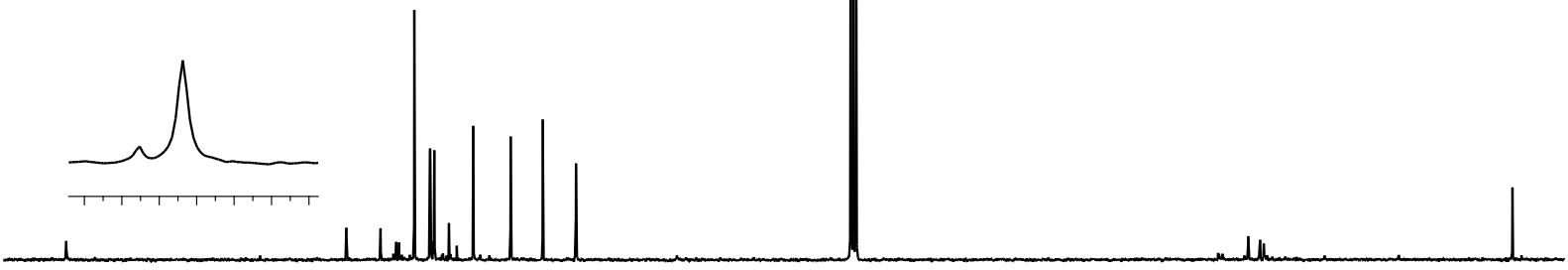

$\begin{array}{llllllllllllllllll}170 & 160 & 150 & 140 & 130 & 120 & 110 & 100 & 90 & 80 & 70 & 60 & 50 & 40 & 30 & 20 & 10 & 0\end{array}$ 


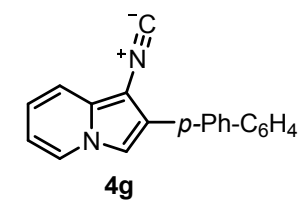

${ }^{1} \mathrm{H} \mathrm{NMR}\left(\mathrm{CDCl}_{3}, 400 \mathrm{MHz}\right)$
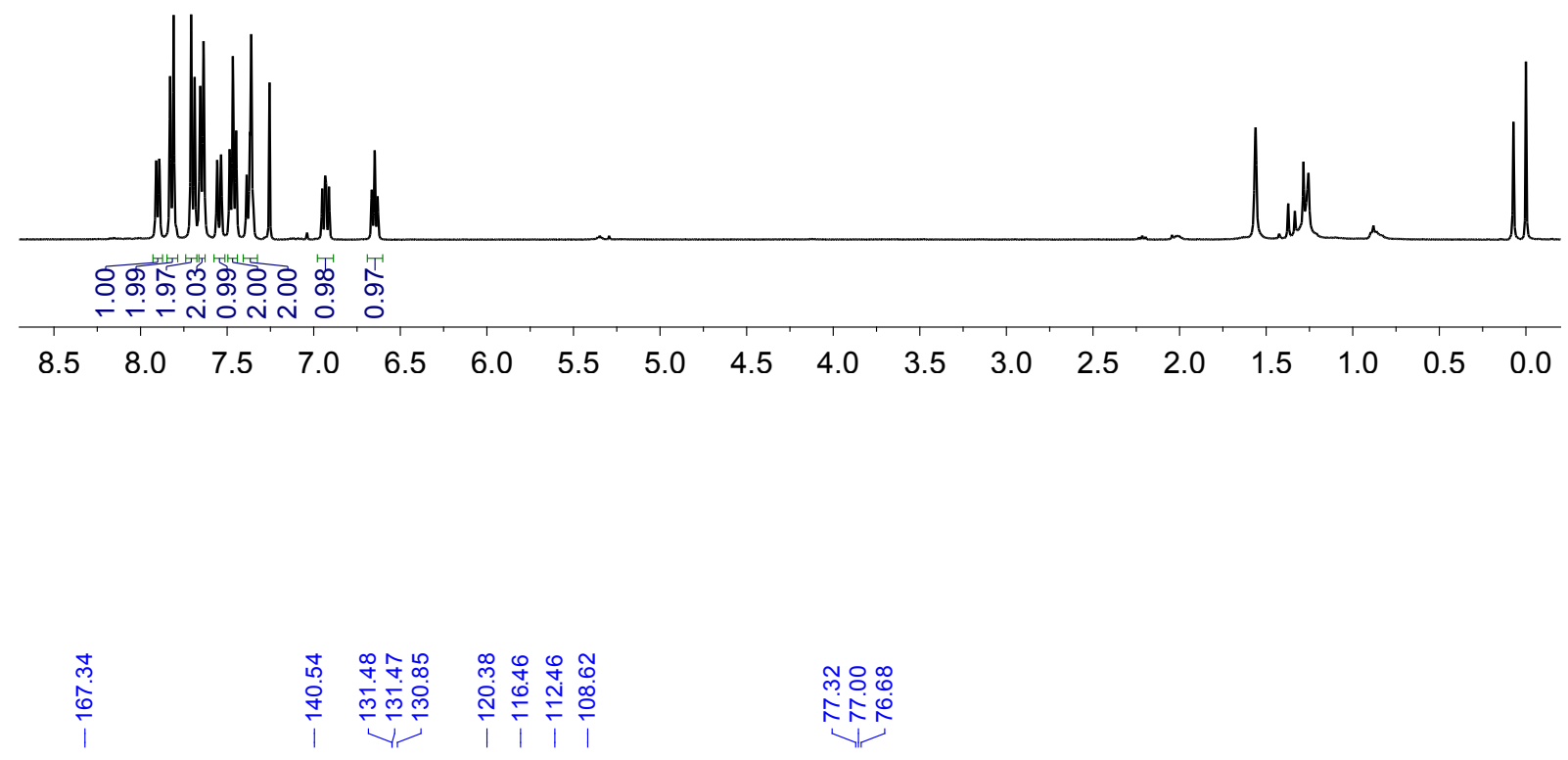

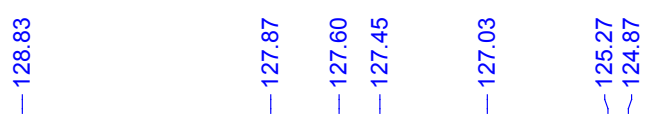
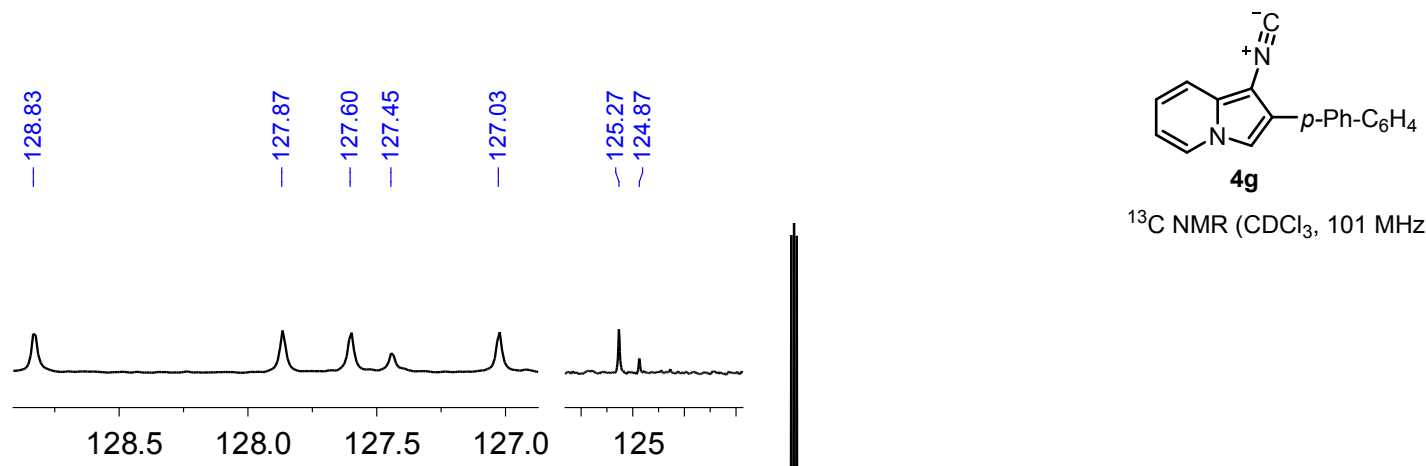

${ }^{13} \mathrm{C} \mathrm{NMR}\left(\mathrm{CDCl}_{3}, 101 \mathrm{MHz}\right)$

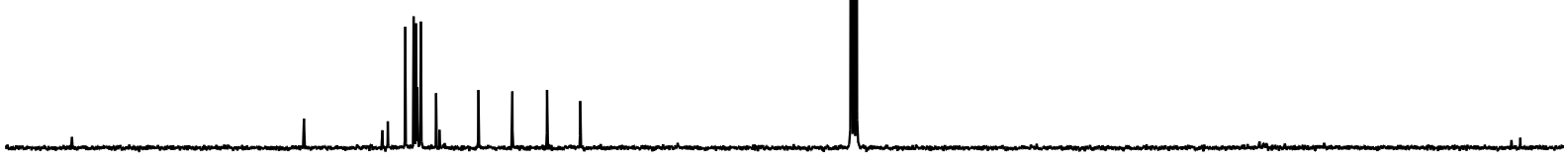

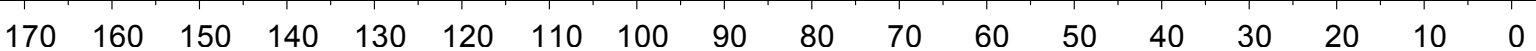




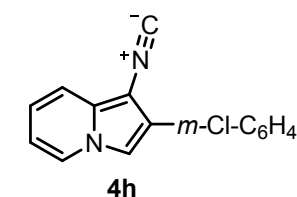

${ }^{1} \mathrm{H}$ NMR $\left(\mathrm{CDCl}_{3}, 400 \mathrm{MHz}\right)$

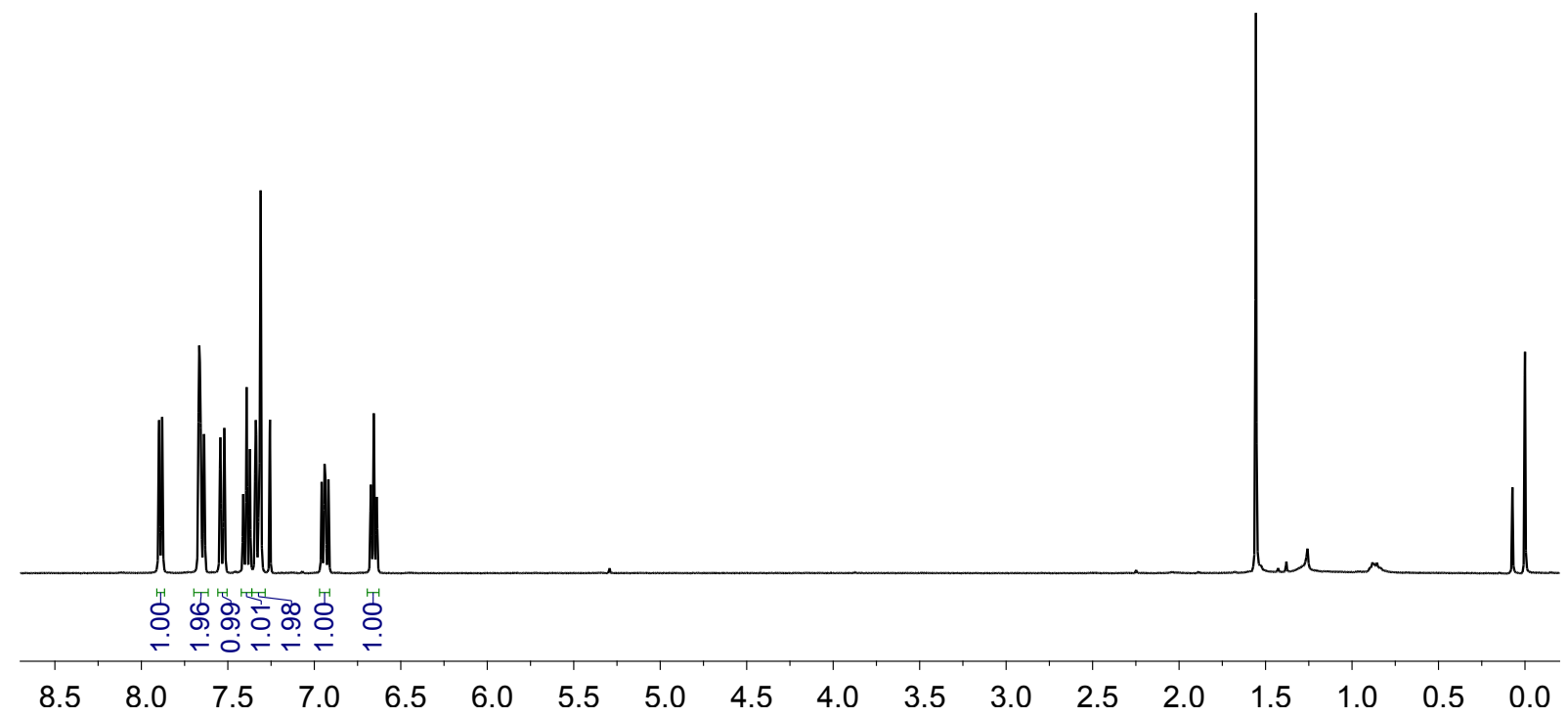

要

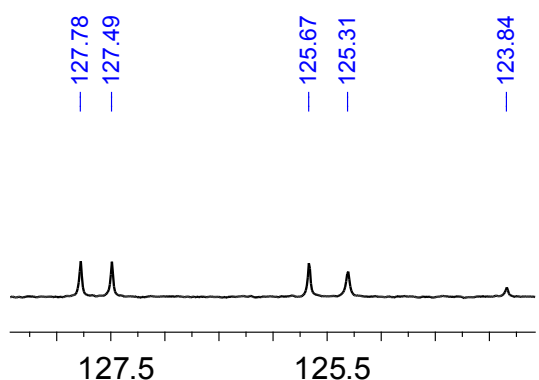

ヘペ

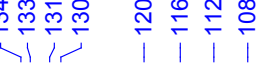

西

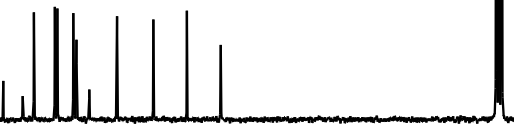

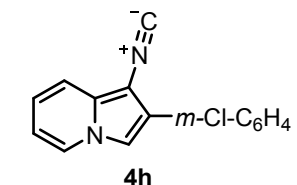

${ }^{13} \mathrm{C} \mathrm{NMR}\left(\mathrm{CDCl}_{3}, 101 \mathrm{MHz}\right)$

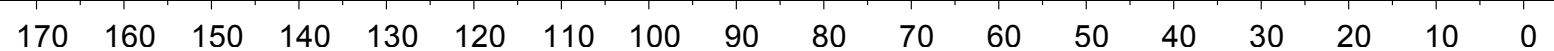




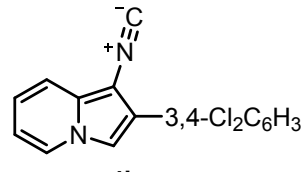

Uld

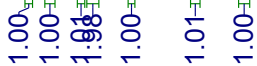

\begin{tabular}{llllllllllllllllll}
\hline 8.5 & 8.0 & 7.5 & 7.0 & 6.5 & 6.0 & 5.5 & 5.0 & 4.5 & 4.0 & 3.5 & 3.0 & 2.5 & 2.0 & 1.5 & 1.0 & 0.5 & 0.0
\end{tabular}
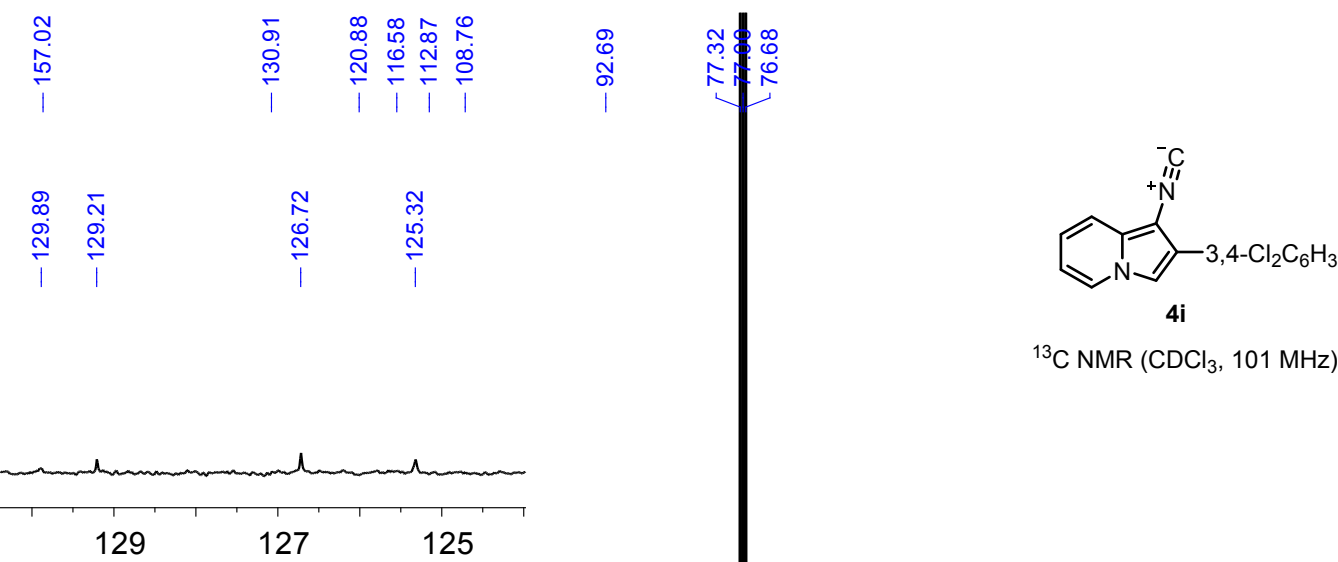

${ }^{13} \mathrm{C} \mathrm{NMR}\left(\mathrm{CDCl}_{3}, 101 \mathrm{MHz}\right)$

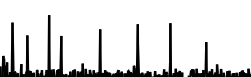

$\begin{array}{lllllllll}170 & 160 & 150 & 140 & 130 & 120 & 110 & 100 & 90\end{array}$ 


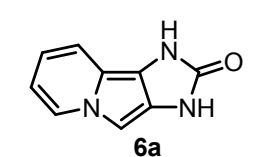

${ }^{1} \mathrm{H}$ NMR $\left(\mathrm{CDCl}_{3}, 400 \mathrm{MHz}\right)$

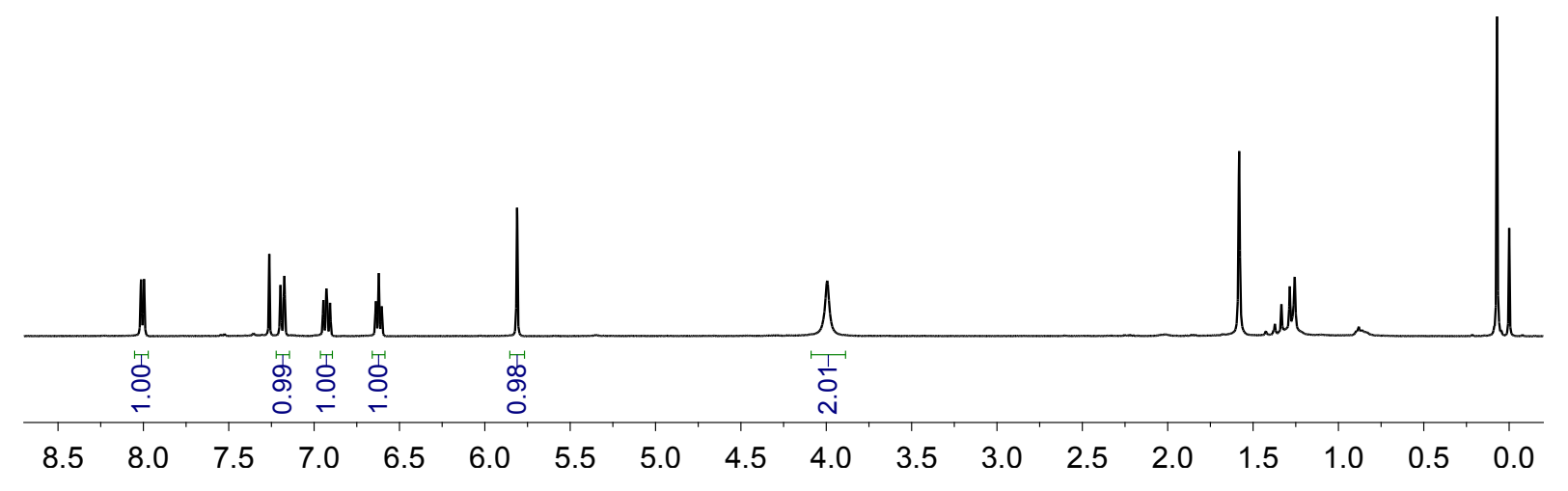

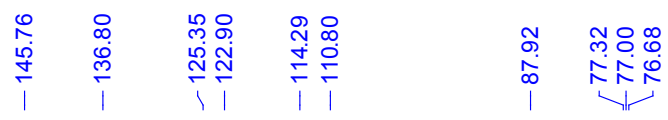
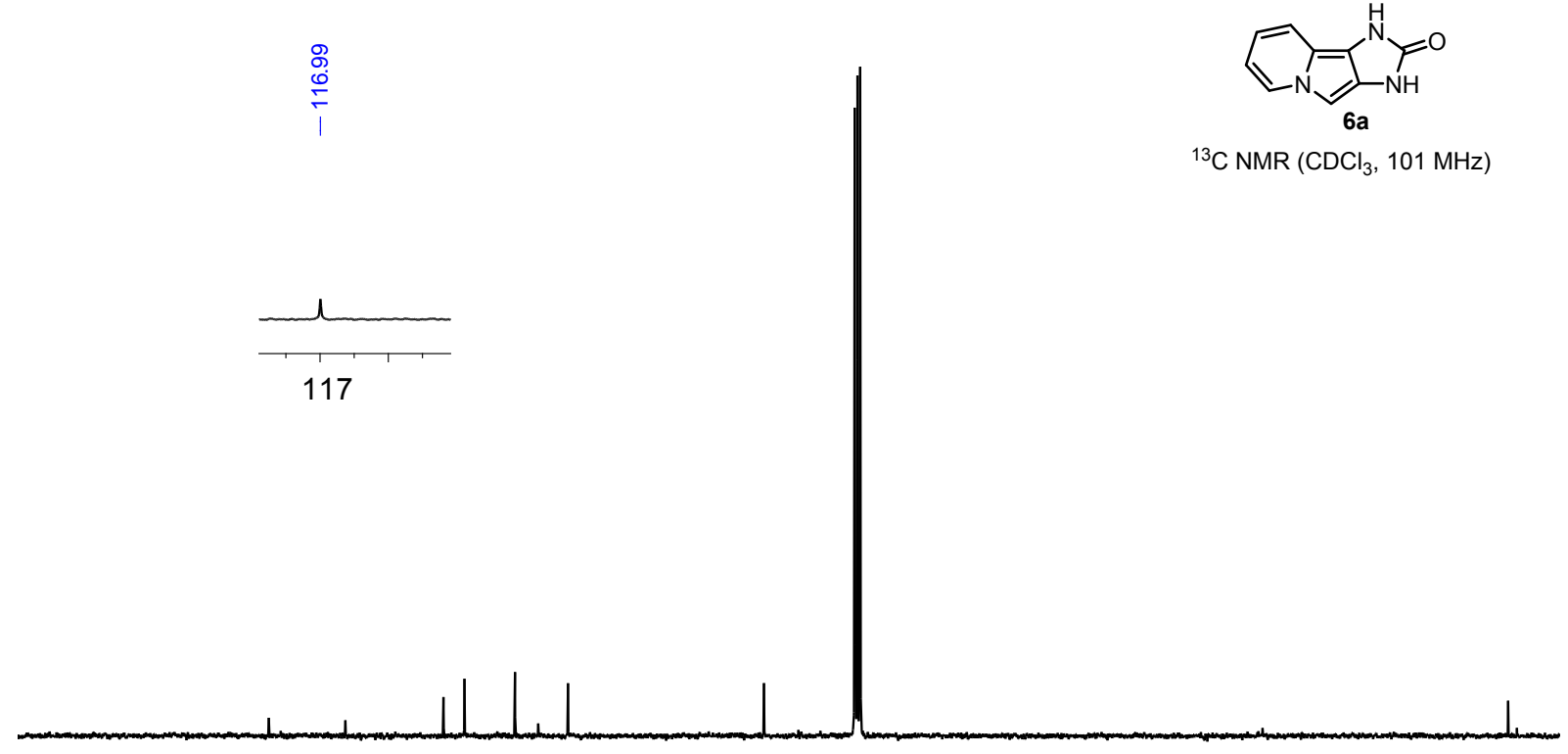

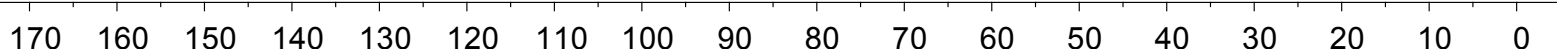




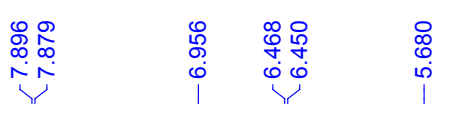

กొ

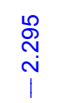

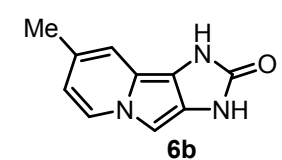

${ }^{1} \mathrm{H}$ NMR $\left(\mathrm{CDCl}_{3}, 400 \mathrm{MHz}\right)$

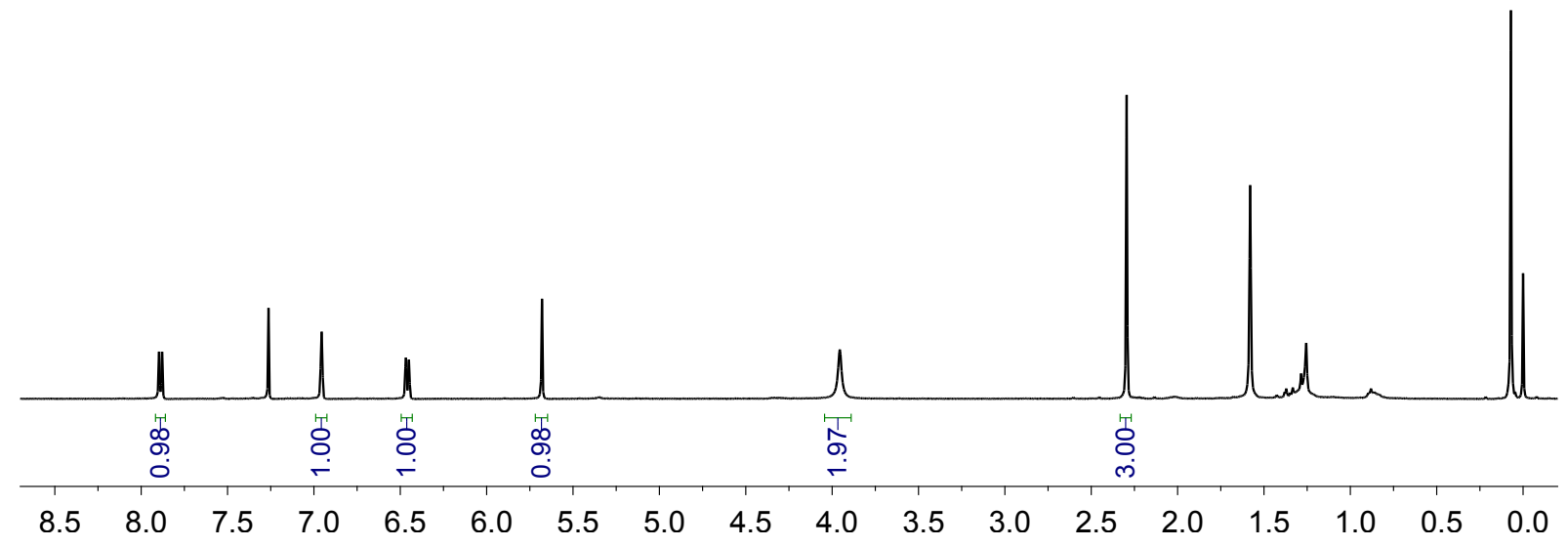




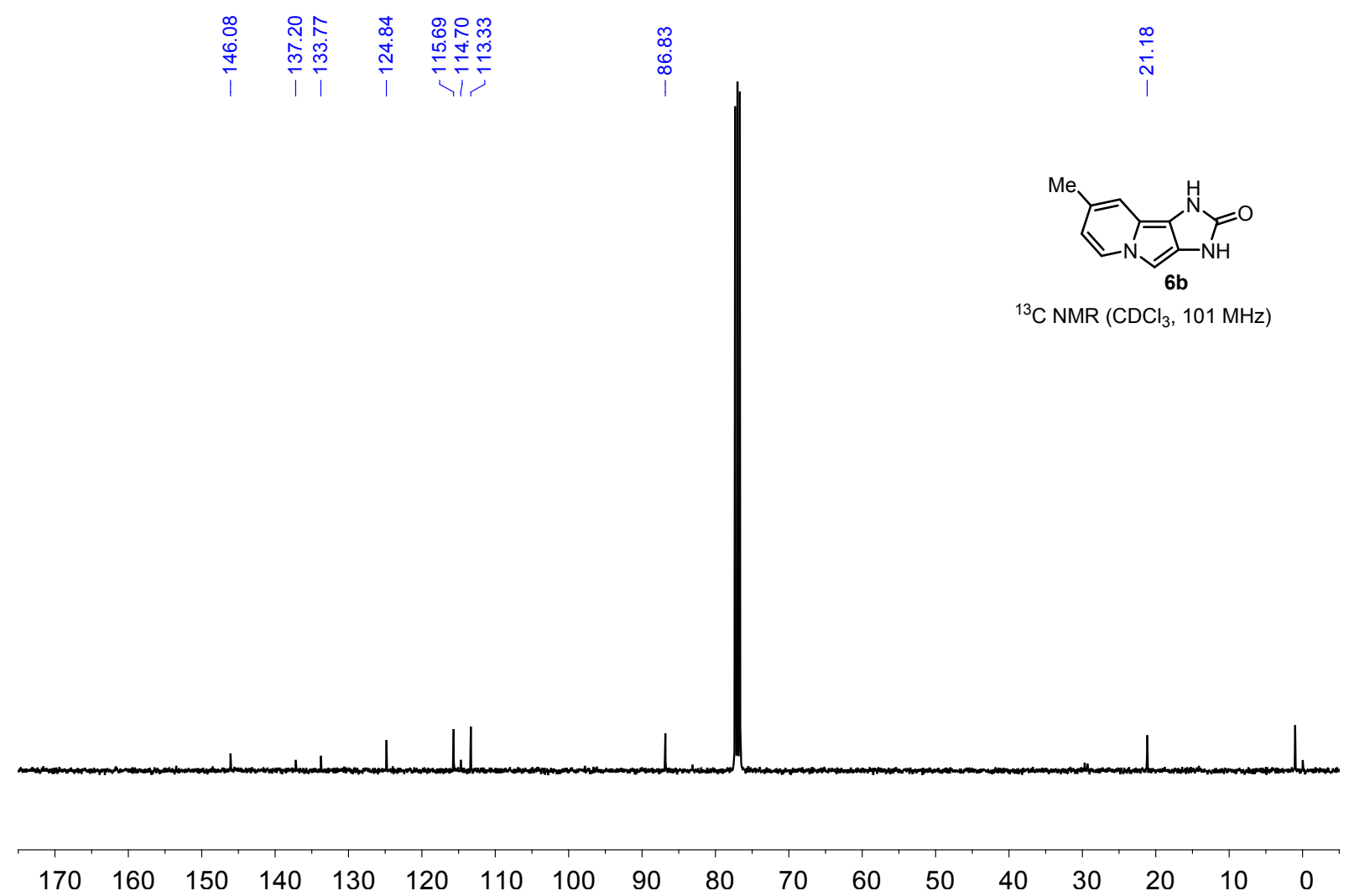




\section{REFERENCES}

(1) Xia, Z.; Zhu, Q. A Transition-Metal-Free Synthesis of Arylcarboxyamides from Aryl Diazonium Salts and Isocyanides. Org. Lett. 2013, 15, 4110-4113.

(2) Qiu, G.; Ding, Q.; Wu, J. Recent Advances in Isocyanide Insertion Chemistry. Chem. Soc. Rev. 2013, 42, 5257-5269.

(3) Piera, J.; Bäckvall, J.-E. Catalytic Oxidation of Organic Substrates by Molecular Oxygen and Hydrogen Peroxide by Multistep Electron Transfer-A Biomimetic Approach. Angew. Chem., Int. Ed. 2008, 47, 3506-3523.

(4) (a) G. M. Sheldrick, SHELXS-97, Program for Solution of Crystal Structures, University of Gottingen, Germany, 1997. (b) G. M. Sheldrick, SHELXL-97, Program for Refinement of Crystal Structures, University of Gottingen, Germany, 1997.

(5) Ghandi M.; Jameà A. H. Pyridine-Mediated, One-Pot, Stereoselective Synthesis of Acyclic Enaminones. Tetrahedron Lett. 2011, 52 4005-4007.

(6) Hou X.; Zhou S.; Li Y.; Guo M.; Zhao W.; Tang X.; Wang G. Synthesis of Indolizines from Pyridinium Salts and Ethyl Bromodifluoroacetate. Org. Lett. 2020, 22, 9313-9318. 